\title{
Totalsynthese von Camptothecin
}

\author{
Dissertation \\ zur Erlangung des Doktorgrades \\ der Mathematisch-Naturwissenschaftlichen Fakultäten \\ der Georg-August-Universität zu Göttingen
}

vorgelegt von

Deshan Liu

aus Huaian

Göttingen 2008 
D7

Referent:

Prof. Dr. Dr. h.c. L. F. Tietze

Korreferent:

Jun.-Prof. Dr. C. Ducho

Tag der mündlichen Prüfung:

01. Juli 2008 
Die vorliegende Arbeit wurde in der Zeit von September 2004 bis März 2008 unter der Leitung von Prof. Dr. Dr. h.c. L. F. Tietze am Institut für Organische und Biomolekulare Chemie der Universität Göttingen angefertigt.

Herrn Prof. Dr. Dr. h.c. L. F. Tietze danke ich für die interessante Themenstellung, die Schaffung hervorragender Arbeitsbedingungen sowie für seine stete Unterstützung und Förderung während des Fortgangs dieser Arbeit. 
Für meine Familie 


\section{INHALTSVERZEICHNIS}

I Einleitung.......................................................................................................................... 1

II Theoretische Grundlagen............................................................................................... 3

1 Der Naturstoff Camptothecin ............................................................................ 3

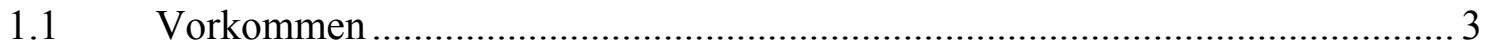

1.2 Entwicklung von Camptothecin und Derivaten................................................. 3

1.3 Ausgewählte Totalsynthese von Camptothecin................................................. 5

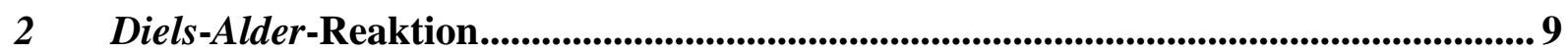

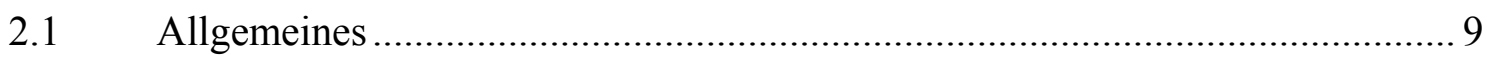

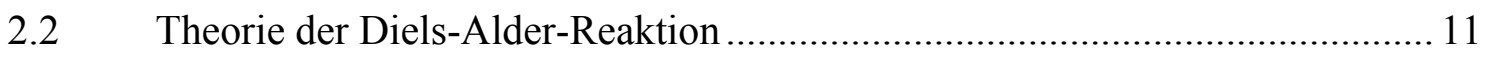

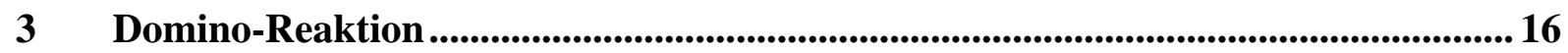

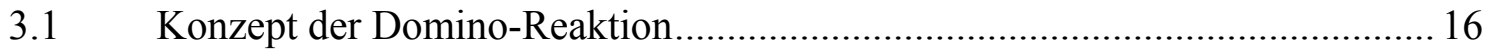

3.2 Domino-Reaktionen in der organischen Synthese............................................ 16

III Aufgabenstellung ..................................................................................................... 20

1 Zielsetzung der Arbeit ..................................................................................................... 20

2 Planung der Arbeit ................................................................................................. 21

IV Darstellung der Ergebnisse ........................................................................................... 22

1 Synthese von Dienophilen .............................................................................................. 22

$1.1 \quad$ Untersuchung zur Synthese des Enolethers 63 ………………………….... 22

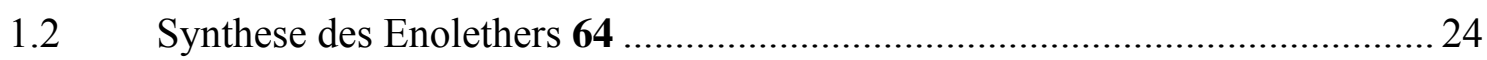

$1.3 \quad$ Untersuchung zur Synthese des Enolethers 65 .............................................. 24

2 Synthese von Aldehyden................................................................................................. 27

2.1 Synthese des Chinolin-Gerüstes ………………………………………….... 27

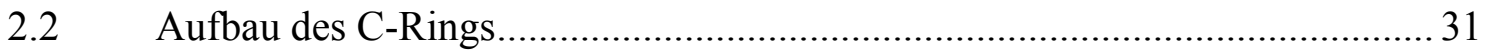

2.2.1 Untersuchung mit verschiedenen Reduktionsmitteln ...................................... 31

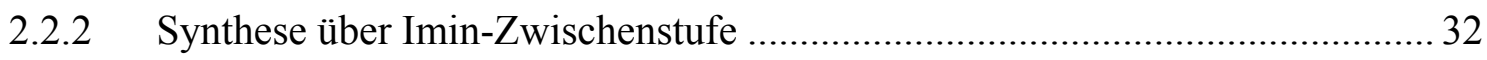

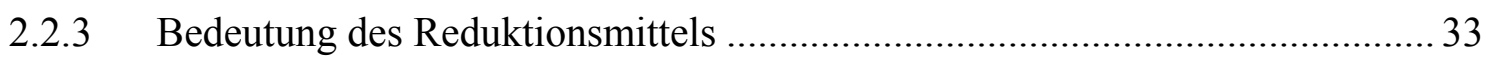

2.2.4 Weitere Optimierung durch Zugabetechnik ...................................................... 33

2.3 Synthese des $N$-Bn-geschützten Aldehyds 94 ............................................... 34 


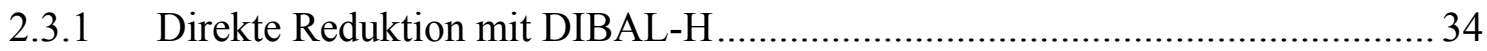

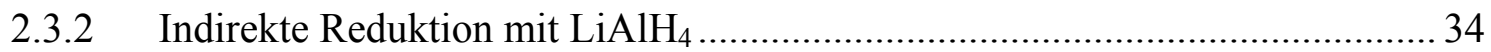

2.4 Synthese des Cbz-geschützten Aldehyds 96 ............................................... 35

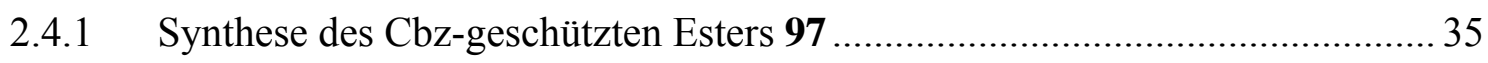

2.4.2 Versuch der Reduktion des $\mathrm{N}$-Cbz-geschützten Esters 97 ............................ 42

2.4.3 Synthese des $N$-Cbz-geschützten Aldehyds 96 durch direkte Umschützung ... 43

2.4.4 Diskussion ausgewählter spektroskopischer Daten der Verbindung 100 ......... 44

2.4.5 Diskussion ausgewählter spektroskopischer Daten der Verbindung 96........... 46

2.5 Synthese der $N$-Troc- und N-ACE-geschützten Aldehyde 114 und 115 ......... 48

3 Durchführung der Domino-Knoevenagel-hetero-Diels-Alder-Reaktion und anschließende Bildung des Lactams.

3.1 Domino-Knoevenagel-hetero-Diels-Alder-Reaktion bei Raumtemperatur und

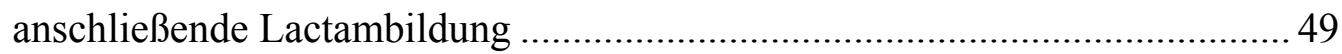

3.1.1 Umsetzung des $N$-Bn-geschützten Aldehyds 94 .......................................... 49

3.1.2 Umsetzung des $\mathrm{N}$-Cbz-geschützten Aldehyds 96 ....................................... 49

3.1.3 Umsetzung des $N$-Troc-geschützten Aldehyds 114 ...................................... 51

3.1.4 Umsetzung des $N$-ACE-geschützten Aldehyds 115 ...................................... 52

3.2 Domino-Knoevenagel-hetero-Diels-Alder-Reaktion bei erhöhter Temperatur und anschließende Lactambildung ............................................................ 53

3.2.1 Feststellung des Lactambildungsproblems .................................................. 53

3.2.2 Durchführung der Domino-Reaktion mit dem $\mathrm{N}$-Cbz-geschützten Aldehyd 96

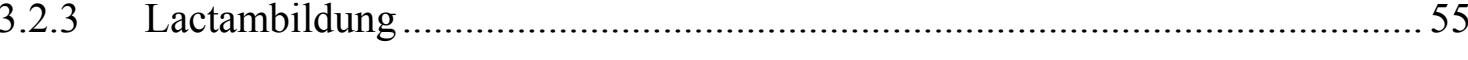

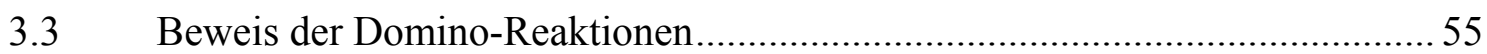

3.4 Mechanismus der Domino-Knoevenagel-hetero-Diels-Alder-Reaktion ........... 56

$4 \quad$ Aufbau des Lactonrings und Synthese von Camptothecin ......................................58

4.1 Untersuchung zur Funktionalisierung des Lactamrings .................................5 58

4.1.1 Aldol-Reaktion der Carbonsäure 129 und des Methylesters 132 .................... 58

4.1.2 Alkylierung des Acetals 134 mit Chlormethylbenzylether .............................60

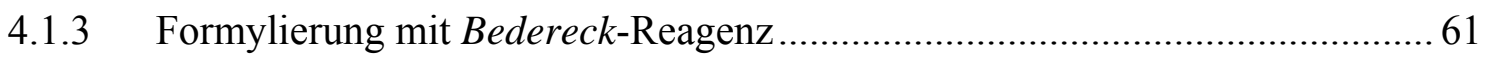

4.2 Untersuchung zur Funktionalisierung des Domino-Produktes......................... 62

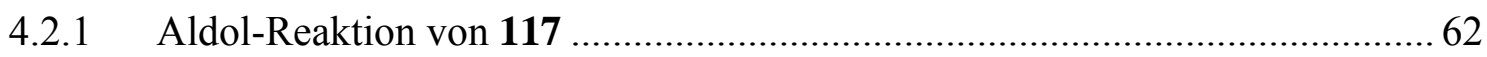

4.2.2 Alkylierung von 117 mit Chlormethylbenzylether....................................... 62

4.2.3 Formylierung von 118 mit Ameisensäureethylester...................................... 63 


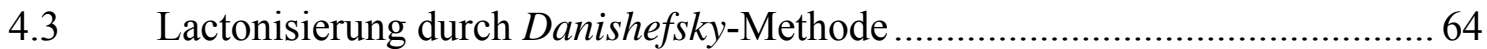

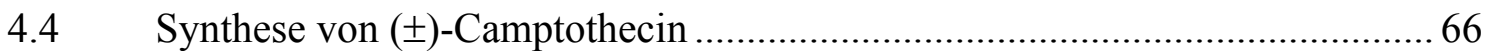

4.4.1 Diskussion ausgewählter spektroskopischer Daten von ( \pm -Camptothecin ..... 66

4.5 Untersuchung zur Synthese von (20S)-Camptothecin.................................... 68

$5 \quad$ Solvolyse-Domino-Reaktion zum Aufbau des Lactonrings ....................................... 70

5.1 Solvolyse-modifizierte Domino-Reaktion und Lactambildung ...................... 70

5.1.1 Optimierung der Methanolyse-Domino-Reaktionen ....................................... 71

5.1.2 Durchführung der Methanolyse-Domino-Reaktion...................................... 73

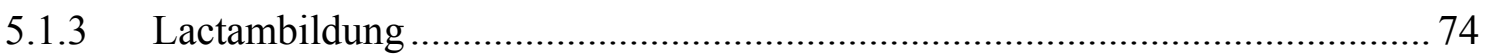

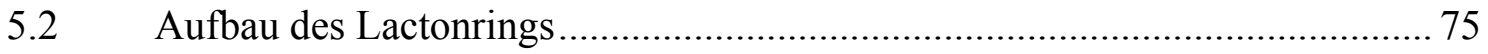

5.2.1 Reduktive Differenzierung zwischen Methylester- und Carboxylgruppe ........ 75

5.2.2 Acetal-Schützung des Aldehyds 62 ......................................................... 77

5.2.3 Regioselektive Reduktion des Methylesters 157....................................... 79

5.2.4 Differenzierung zwischen Methylester- und tert-Butylestergruppe ................. 87

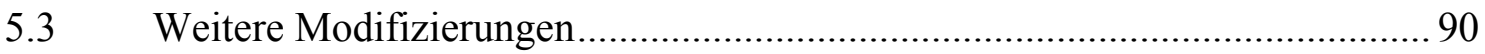

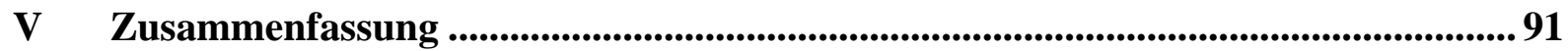

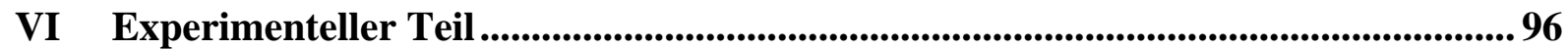

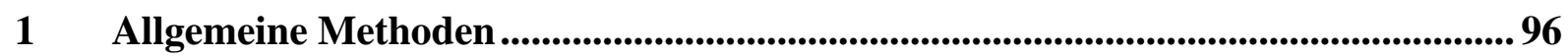

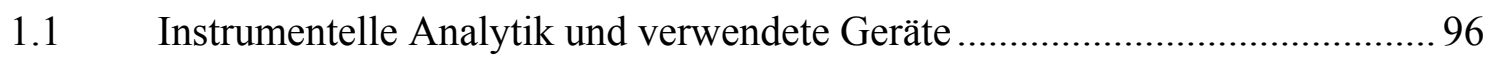

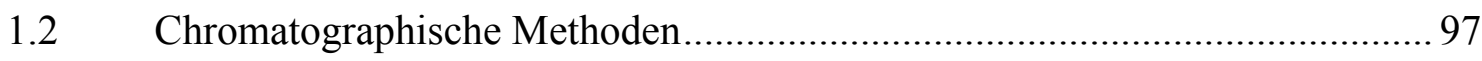

2 Synthese von Dienophilen .....................................................................................................98

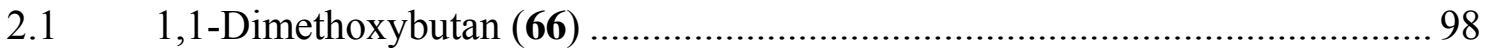

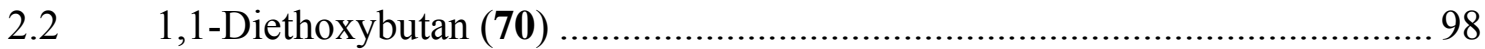

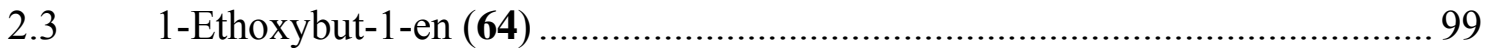

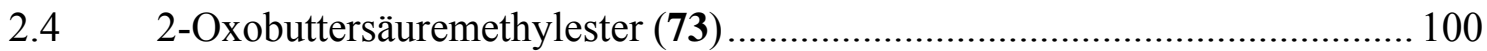

$2.5 \quad$ 2-Hydroxybuttersäuremethylester (74) ....................................................... 101

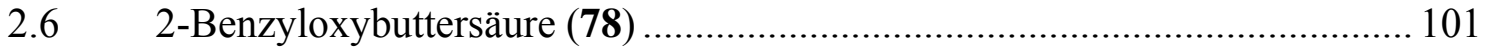

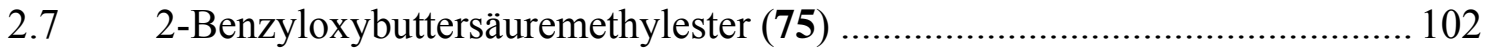

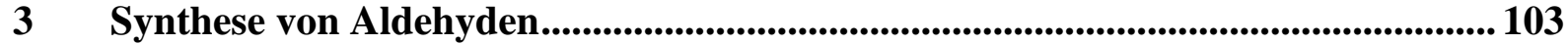

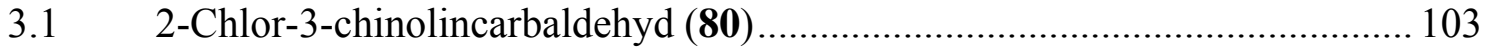

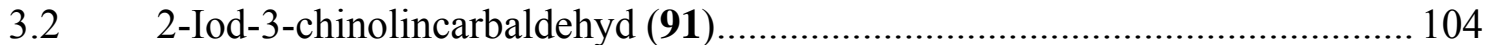

3.3 (E)-3-(3-Formylchinolin-2-yl)acrylsäureethylester (81) ............................. 105 
3.4 (2-Benzyl-2,3-dihydro-1H-pyrrolo[3,4-b]chinolin-3-yl)-essigsäure-ethylester (93). 106

3.5 (2-(4-Methoxybenzyl)-2,3-dihydro-1H-pyrrolo[3,4-b]chinolin-3-yl)essigsäureethylester (100).

3.6 (2-Benzyl-2,3-dihydro-1H-pyrrolo[3,4-b]chinolin-3-yl)-acetaldehyd (94)... 108

3.7 (2-Benzyloxycarbonyl-2,3-dihydro-1H-pyrrolo[3,4-b]chinolin-3-yl)acetaldehyd (96) 109

3.8 (2-2,2,2-Trichlor-ethoxycarbonyl-2,3-dihydro-1H-pyrrolo[3,4-b]chinolin-3-yl)acetaldehyd (114)

3.9 (2-(4-Methoxybenzyl)-2,3-dihydro-1H-pyrrolo[3,4-b]chinolin-3-yl)acetaldehyd (71)

3.10 (2-1-Chlor-ethoxycarbonyl-2,3-dihydro-1H-pyrrolo[3,4-b]chinolin-3-yl)acetaldehyd (115)

3.11 2-(2-Benzyl-2,3-dihydro-1H-pyrrolo[3,4-b]chinolin-3-yl)-Ethanol (95) ....... 115

3.12 (2-2,2,2-Trichlor-ethoxycarbonyl-2,3-dihydro-1H-pyrrolo[3,4-b]chinolin-3-yl)Essigsäureethylester (110) 116

3.13 (2-Benzyloxycarbonyl-2,3-dihydro-1H-pyrrolo[3,4-b]chinolin-3-yl)Essigsäureethylester (97)

3.14 (2,3-dihydro-1H-pyrrolo[3,4-b]chinolin-3-yl)-Essigsäureethylester (98).

4 Durchführung der Domino-Knoevenagel-hetero-Diels-Alder-Reaktion und anschließende Bildung des Lactams

4.1 4-(2-Benzyloxycarbonyl-2,3-dihydro-1H-pyrrolo[3,4-b]chinolin-3-ylmethyl)6-ethoxy-5-ethyl-tetrahydropyran-2-on (117)

4.2 4-(2-2,2,2-Trichlor-ethoxycarbonyl-2,3-dihydro-1H-pyrrolo[3,4-b]chinolin-3ylmethyl)-6-ethoxy-5-ethyl-tetrahydropyran-2-on (118)

4.3 2-(9-Oxo-5b,6,7,8,9,11-hexahydro-indolizino[1,2-b]chinolin-7-yl)butyraldehyd (61)

5 Aufbau des Lactonrings und Synthese von Camptothecin 123

5.1 2-(9-Oxo-5b,6,7,8,9,11-hexahydro-indolizino[1,2-b]chinolin-7-yl)-buttersäure (129).

5.2 7-Propionyl-11H-indolizino[1,2-b]chinolin-9-on (123) 124

5.3 2-(9-Oxo-9,11-dihydro-indolizino[1,2-b]chinolin-7-yl)-butyraldehyd (122) 125

5.4 2-(9-Oxo-5b,6,7,8,9,11-hexahydro-indolizino[1,2-b]chinolin-7-yl)buttersäuremethylester (132) 126 
5.5 2-(9-Oxo-9,11-dihydro-indolizino[1,2-b]chinolin-7-yl)-buttersäuremethylester (20).

5.6 20-Desoxycamptothecin (141) 128

5.7 4-Ethyl-4-hydroxymethyl-1,7-dihydro-4H-2-oxa-6a,13-diaza-dibenzo[b,g]fluoren-3,6-dion (142)

5.8 2-(9-Oxo-9,11-dihydro-indolizino[1,2-b]chinolin-7-yl)-buttersäure (143).... 130

$5.9 \quad( \pm)$-Camptothecin (11) 131

6 Methanolyse-modifizierte Domino-Knoevenagel-hetero-Diels-Alder-Reaktion und Aufbau des Lactonrings

6.1 4-(2-Benzyloxycarbonyl-2,3-dihydro-1H-pyrrolo[3,4-b]chinolin-3-ylmethyl)6-ethoxy-5-ethyl-3-methyloxycarbonyl-tetrahydropyran-2-on (146)

6.2 4-(2-2,2,2-Trichlor-ethoxycarbonyl-2,3-dihydro-1H-pyrrolo[3,4-b]chinolin-3ylmethyl)-6-ethoxy-5-ethyl-3-methyloxycarbonyl-tetrahydropyran-2-on (126)

6.3 2-(9-Oxo-8-methyloxycarbonyl-5b,6,7,8,9,11-hexahydro-indolizino[1,2-b]chinolin-7-yl)-butyraldehyd (62)

6.4 2-(9-Oxo-8-methyloxycarbonyl-5b,6,7,8,9,11-hexahydro-indolizino[1,2-b]chinolin-7-yl)-buttersäure (147)

6.5 2-(9-Oxo-8-methyloxycarbonyl-5b,6,7,8,9,11-hexahydro-indolizino[1,2-b]chinolin-7-yl)-buttersäuremethylester (148).

6.6 2-(8-Methyloxycarbonyl-9-oxo-9,11-dihydro-indolizino[1,2-b]chinolin-7-yl)buttersäuremethylester (157)

6.7 2-(8-Formyl-9-oxo-9,11-dihydro-indolizino[1,2-b]chinolin-7-yl)-buttersäuremethylester (158).

6.8 2-(8-Isobutyloxycarbonyl-9-oxo-9,11-dihydro-indolizino[1,2-b]chinolin-7-yl)buttersäuremethylester (160) 138

6.9 8-Methyloxycarbonyl-7-(1-dimethoxymethyl-propyl)-5b,7,8,11-tetrahydro-6Hindolizino[1,2-b]chinolin-9-on (151).

6.10 8-Methyloxycarbonyl-7-(1-dimethoxymethyl-propyl)-11H-indolizino[1,2-b]chinolin-9-on (152).

6.11 8-Hydroxymethyl-7-(1-dimethoxymethyl-propyl)-11H-indolizino[1,2-b]chinolin-9-on (154).

6.12 8-Hydroxymethyl-7-(1-methoxymethylen-propyl)-11H-indolizino[1,2-b]chinolin-9-on (155) 
6.13 4-Ethyl-3-hydroxy-1,3,4,12-tetrahydro-2-oxa-6,12a-diaza-dibenzo[b,h]fluoren-13-on (156)

6.14 2-(9-Oxo-8-methyloxycarbonyl-5b,6,7,8,9,11-hexahydro-indolizino[1,2-b]chinolin-7-yl)-buttersäure-tert-butylester (161)

6.15 2-(8-Methyloxycarbonyl-9-oxo-9,11-dihydro-indolizino[1,2-b]chinolin-7-yl)buttersäure-tert-butylester (162)

6.16 2-(8-Formyl-9-oxo-9,11-dihydro-indolizino[1,2-b]chinolin-7-yl)-buttersäuretert-butylester (163) 146

6.17 2-(8-Hydroxymethyl-9-oxo-9,11-dihydro-indolizino[1,2-b]chinolin-7-yl)buttersäure-tert-butylester (164)

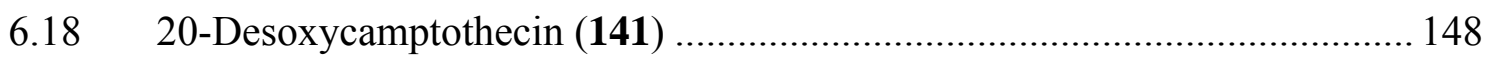

6.19 4-Ethyl-12H-2-oxa-6,12a-diaza-dibenzo[b,h]fluoren-1,13-dion (165) .......... 149

VII Anhang....................................................................................................................................... 150

1 Verwendete Abkürzungen und Akronyme.............................................................. 150

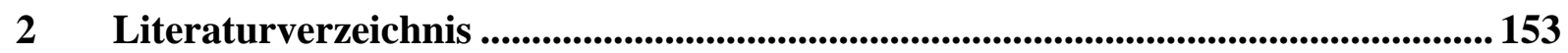

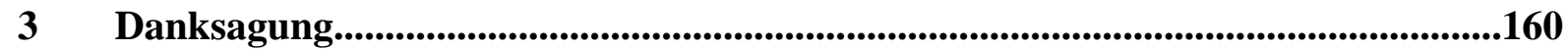

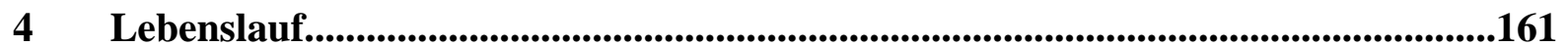




\section{EINLEITUNG}

Die Chemie ist wie keine andere Naturwissenschaft ein allgegenwärtiger und unverzichtbarer Bestandteil unseres Lebens geworden. Innovative Entwicklungen in den Bereichen Pharmazie, Lebensmittel- und Kunststoffchemie sowie nicht zuletzt im Pflanzenschutz und der Düngemittelindustrie haben nicht nur zur hohen Lebensqualität der hochtechnisierten Nationen beigetragen, sondern liefern auch Voraussetzungen für die Befriedigung elementarer Grundbedürfnisse der gesamten Menschheit. Ein wichtiges Teilgebiet der Chemie stellt die organische Synthese dar, deren Grundstein im Jahr 1828 durch die Harnstoffsynthese von F. Wöhler gelegt wurde. ${ }^{1}$ Einhergehend mit immer besseren Methoden hat sich die Synthesechemie seitdem explosionsartig entwickelt. Besonders im 20. Jahrhundert haben viele anspruchsvolle Totalsynthesen wie z. B. von Vitamin $\mathrm{B}_{12}{ }^{2}$, Palytoxin ${ }^{3}$, Brevetoxin ${ }^{4}$ und Vancomycin $^{5}$ den Beweis erbracht, dass jedes noch so komplexe Molekül synthetisch zugänglich ist.

Die chemische Synthese erweist sich für die Wirkstoffentwicklung im pharmazeutischen Bereich als unverzichtbar. Wirkstoffe können direkt aus Pflanzen, Schwämmen oder Mikroorganismen meist nur in geringen Mengen isoliert werden. Aufgabe des Chemikers ist es somit, einen möglichst kurzen Syntheseweg zu diesen Substanzen zu finden, so dass ihre industrielle Produktion in den benötigten Mengen realisiert werden kann. Ferner kommt vor allem eine chemische Derivatisierung der von der Natur vorgegebenen Leitstrukturen in Betracht, wenn die Verträglichkeit oder Wirksamkeit von natürlichen Wirkstoffen verbessert werden muss. Ein klassisches Beispiel ist die Entwicklungen von Aspirin ${ }^{\circledR},{ }^{6}$ dem heutzutage erfolgreichsten Medikament. Während die Schmerz stillende und Fieber senkende Wirkung der aus Weidenrinde gewonnenen Salicylsäure bereits in der traditionellen Medizin der Maya bekannt war, erwies sich die orale Verträglichkeit doch als sehr schlecht. Durch eine einfache Acetylierung der Hydroxygruppe konnten die pharmakologischen Eigenschaften erheblich verbessert werden. Ein anderes Beispiel ist die Entwicklung von Camptothecin-Derivaten wie Irinotecan und Topotecan, ${ }^{7}$ die in der Krebstherapie klinisch eingesetzt werden. Das aus der Pflanze Camptotheca acuminata gewonnene Alkaloid Camptothecin zeigt zwar cytotoxische Wirkung, findet aber aufgrund geringer Wasserlöslichkeit und $\mathrm{zu}$ hoher Toxizität keine klinische Anwendung. Die Einführung bestimmter funktionellen Gruppen am Chinolinring (siehe II Theoretische Grundlagen, Kap. 1) kann die Löslichkeit und das pharmakologische Profil deutlich verbessern. Die zwei daraus resultierenden Derivate Irinotecan (Camptosar ${ }^{\circledR}$ ) 
and Topotecan $\left(\right.$ Hycamptin $^{\circledR}$ ) werden für die Therapie verschiedenen Tumoren angewandt. Mehrere ähnliche Verbindungen befinden sich zur Zeit in klinischen Phasen.

Allerdings beschränken sich viele der veröffentlichten Synthesen auf die industrielle Anwendung. Eine Synthese, welche neben akademischem auch wirtschaftliches Interesse hervorrufen soll, muss sich auch im Hinblick auf ihre Effizienz, Ökobilanz, Ökonomie und Atomökonomie messen lassen. Hierbei kann das von L.F. Tietze entwickelte DominoKonzept von erheblichen Nutzen sein. Bei Domino-Prozessen erfolgen mehrere Reaktionsschritte sequenziell unter gleichen Reaktionsbedingungen, wobei die nachfolgenden Reaktionen stets an den im vorherigen Reaktionsschritt gebildeten Funktionalitäten stattfinden. Eine solche Reaktionsführung ist sowohl aus ökonomischer als auch aus ökologischer Sicht von Vorteil, da sowohl die erforderliche Menge an Reagenzien und Lösungsmitteln als auch die entstehende Menge an Abfällen reduziert werden. ${ }^{8}$

In der vorliegenden Arbeit werden die in der Arbeitsgruppe von L. F. Tietze entwickelte Domino-Knoevenagel-hetero-Diels-Alder-Reaktion ${ }^{9}$ zur Totalsynthese von Camptothecin angewandt. Dies ermöglicht einen kurzen und effizienten Weg zur Zielverbindung und erlaubt auch eine Vielzahl an Analoga herzustellen. 


\section{THEORETISCHE GRUNDLAGEN}

\section{Der Naturstoff Camptothecin}

\subsection{Vorkommen}

Vor ca. funfzig Jahren startete das amerikanische National Cancer Institute (NCI) ein Programm, um die antitumorale Wirksamkeit von Verbindungen einschließlich 1000 Pflanzenextrakten zu überprüfen. ${ }^{10}$ Darunter war ein aus Blättern des chinesischen Xi ShuBaums (Camptotheca acuminata) gewonnener Rohextrakt, der als einziger Extrakt hoch antitumorale Aktivität aufwies.
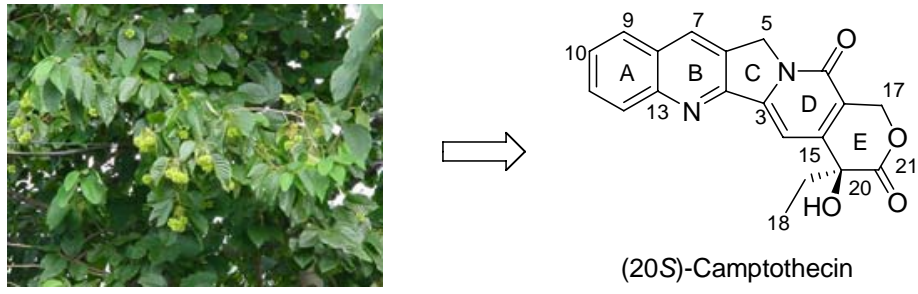

(20S)-Camptothecin

Abbildung 1. Camptotheca acuminata und (20S)-Camptothecin

Durch Röntgenstrukturanalyse konnten 1966 M. E. Wall und M. C. Wani die wirksame Komponente strukturell aufklären. ${ }^{11}$ Es handelt sich um ein pentacyclisches Alkaloid mit einem Pyrrochinolin-Ringsystem (Ring A-C), einer Pyridon-Komponente (Ring D) und einer Lacton-Einheit (Ring E) mit einem stereogenen Zentrum, das eine S-Konfiguration aufweist (siehe Abbildung 1). In Anlehnung an den botanischen Namen wird der Naturstoff als (20S)-Camptothecin (CPT) bezeichnet.

\subsection{Entwicklung von Camptothecin und Derivaten}

Aufgrund der sehr geringeren Wasserlöslichkeit von CPT $(0.0025 \mathrm{mg} / \mathrm{ml})^{12}$ wurden Ende der sechziger Jahre die ersten klinischen Studien mit dem wasserlöslichen Natriumsalz (mit geöffnetem Lactonring) durchgeführt. Bei der Applikation beobachte man eine geringe Effizienz und eine zu hohe, nicht tolerable Toxizität, die zum frühzeitigen Abbruch der Studie führten. Erst 1985 wurde die neuartige Wirkung von CPT als DNA Typ I TopoisomeraseInhibitor entdeckt. ${ }^{13}$ Dies hatte ein wachsendes Interesse der Wissenschaft, verbunden mit weitergehenden Studien zur Synthese ${ }^{14}$ und Wirkung ${ }^{15}$ von CPT, zur Folge. 
Studien zu Struktur-Wirkungs-Beziehungen zeigten, dass der Lactonring intakt bleiben muss und Derivatisierungen an C-7, C-9 und C-10 keinen Einfluss auf die biologischen Eigenschaften haben. ${ }^{16}$ Dem zufolge konnte man maßgeschneiderte CPT-Derivate synthetisieren, die wasserlöslich sind und ein verbessertes Pharmaprofil zeigten. Als erste Generation von CPT-Derivaten ließen sich Topotecan $^{17}$ und Irinotecan ${ }^{18}$ ausgehend von Camptothecin semi-synthetisch herstellen. Ende der neunziger Jahre wurden Topotecan (Hycamptin $^{\circledR}$, TPT) und Irinotecan (Camptosar ${ }^{\circledR}$, CPT-11) zur Therapie verschiedener Turmoren wie z. B. Ovarian- und Colonkrebs zugelassen und von GlaxoSmithKline und Pharmacia (nun Pfizer) vermarket. Sie wurden auch zur Behandlung von Lungen- und Colorectal-Krebs unter Kombination mit anderen Antikrebsmittel klinisch eingesetzt. ${ }^{19}$ Während beide Präparate Nebenwirkungen wie Myelosuppression, Nierentoxizität und Diarrhöen aufweisen, zeigen mehrere neue Derivate wie Silatecan oder Rubitecan (siehe Abbildung 3) bessere Ergebnisse. Sie sind zur Zeit als zweite Generation in der klinischen Phase-I/II. $^{20}$

Inzwischen ist bekannt, dass unter physiologischen Bedingungen das Gleichgewicht zwischen geschlossenem und offenkettigem E-Ring von CPT schnell auf die Seite der Carboxylatform verschoben wird (siehe Abbildung 2). ${ }^{21}$ Da Öffnung des Lactonrings zur erheblichen Reduzierung der antitumoralen Wirksamkeit und vielfachen Erhöhung der Toxizität führte, ${ }^{22}$ wurde eine Lactonstabilisierungsstrategie besonders durch 20-O-Veresterung und 20-OKonjugate entwickelt. ${ }^{23}$ Als Ergänzungen zu etablierten Struktur-Wirkungs-Beziehungen werden auch Modifizierung des E-Rings ${ }^{24}$ und 14-Aza-camptothecinderivate ${ }^{25}$ untersucht.

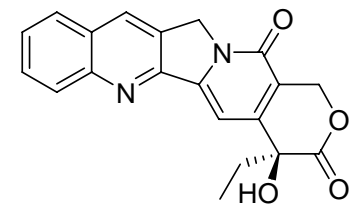

(20S)-Camptothecin

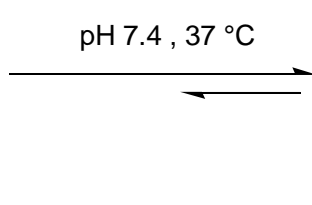

$\begin{aligned} \text { Carboxylatform: } & >80 \% \\ & >99 \%(+ \text { HSA })\end{aligned}$

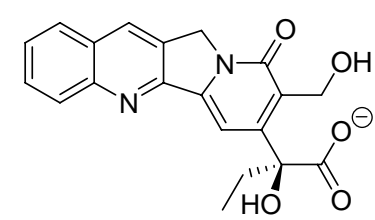

Abbildung 2. Reversible Öffnung des Lactonrings von CPT unter physiologischen Bedingungen ( $\mathrm{pH}=7.4 \mathrm{mit}$ PBS-Puffer, $37^{\circ} \mathrm{C}$, HSA: Humanserumalbumin) 


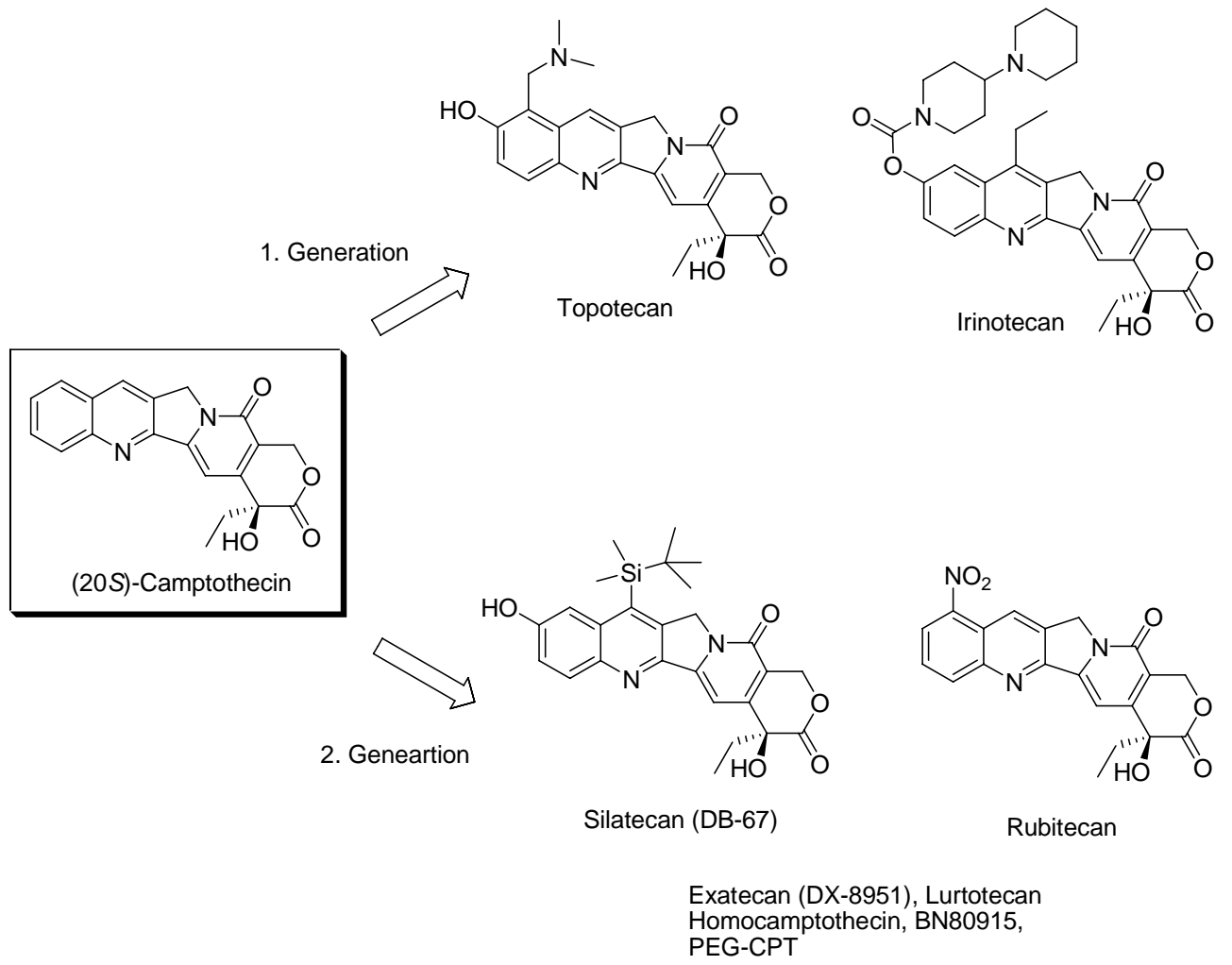

Abbildung 3. Entwicklung von CPT-Derivaten

\subsection{Ausgewählte Totalsynthese von Camptothecin}

Die erkannte medizinische Bedeutung, die interessante pentacyclische Struktur sowie das seltene natürliche Vorkommen von CPT haben seit seiner Entdeckung das Interesse synthetisch arbeitender Chemiker auf diesen Naturstoff gelenkt. Die erste Totalsynthese wurde im Jahre 1971 von G. Stork publiziert. ${ }^{26}$ Die Friedlander Kondensation des o-Aminobenzaldehyds (1) mit dem Pyrrolindon 2 lieferte die Carbonsäure 3, die sich durch anschließende Hydrolyse sowie Veresterung und Amidierung in das Diesteramid 5 überführen ließ (siehe Schema 1). Die weitere NaOEt-induzierte Cylisierung und nachfolgende Hydrolyse mit gleichzeitiger Decarboxylierung lieferten das $\beta$-Ketoamid 6. Die Reduktion von 6 mit $\mathrm{NaBH}_{4}$ und Acetylierung mit Essigsäureanhydrid stellten das Schlüsselfragment 7 bereit. In einer Additions-Cyclisierungs-Reaktion mit dem Lithtiumsalz von 8 wurde 7 in das Lacton 9 mit 85\% Ausbeute umgewandelt. Die weitere Hydrolyse, Reduktion, Acetylierung und DDQ-Dehydrierung lieferten das Pyridon 10. Durch die nachfolgende Deacetylierung, Reduktion und Lactonisierung wurde die erste Totalsynthese von $( \pm)$-Camptothecin verwirklicht. 


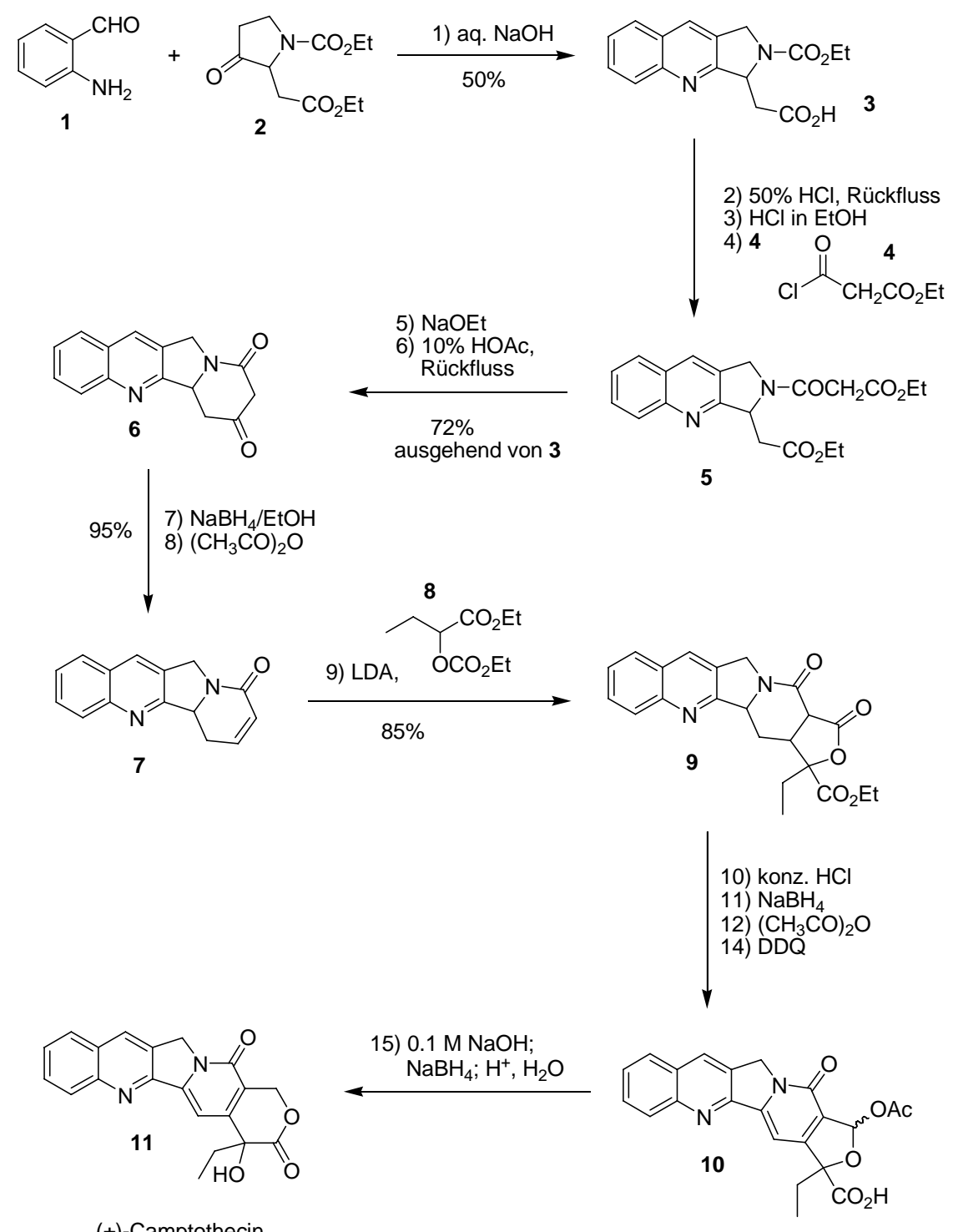

( \pm )-Camptothecin

Schema 1. Die erste Totalsynthese von ( \pm )-Camptothecin durch Addition-Cyclisierungsstrategie

D.P. Curran bediente sich einer radikalischen Annelierung zur Totalsynthese von ( \pm )-Camptothecin (siehe Schema 2). ${ }^{27}$ Die Umsetzung von 12 mit $\mathrm{PCl}_{5}$, gasförmigen $\mathrm{HBr}$ und Methanol lieferte das Brompyridon 13, das durch die weitere $N$-Propargylierung und a-Alkylierung in 15 überführt wurde. In der (4+1)-radikalischen Annelierung von 15 mit Phenylisonitril unter Lichtbestrahlung ließ sich über 5-exo-Cyclisierung der Ester 20 in 45\% Ausbeute darstellen. Über weitere zwei literaturbekannte Stufen konnte 20 in (士)-Camptothecin umgewandelt werden. 

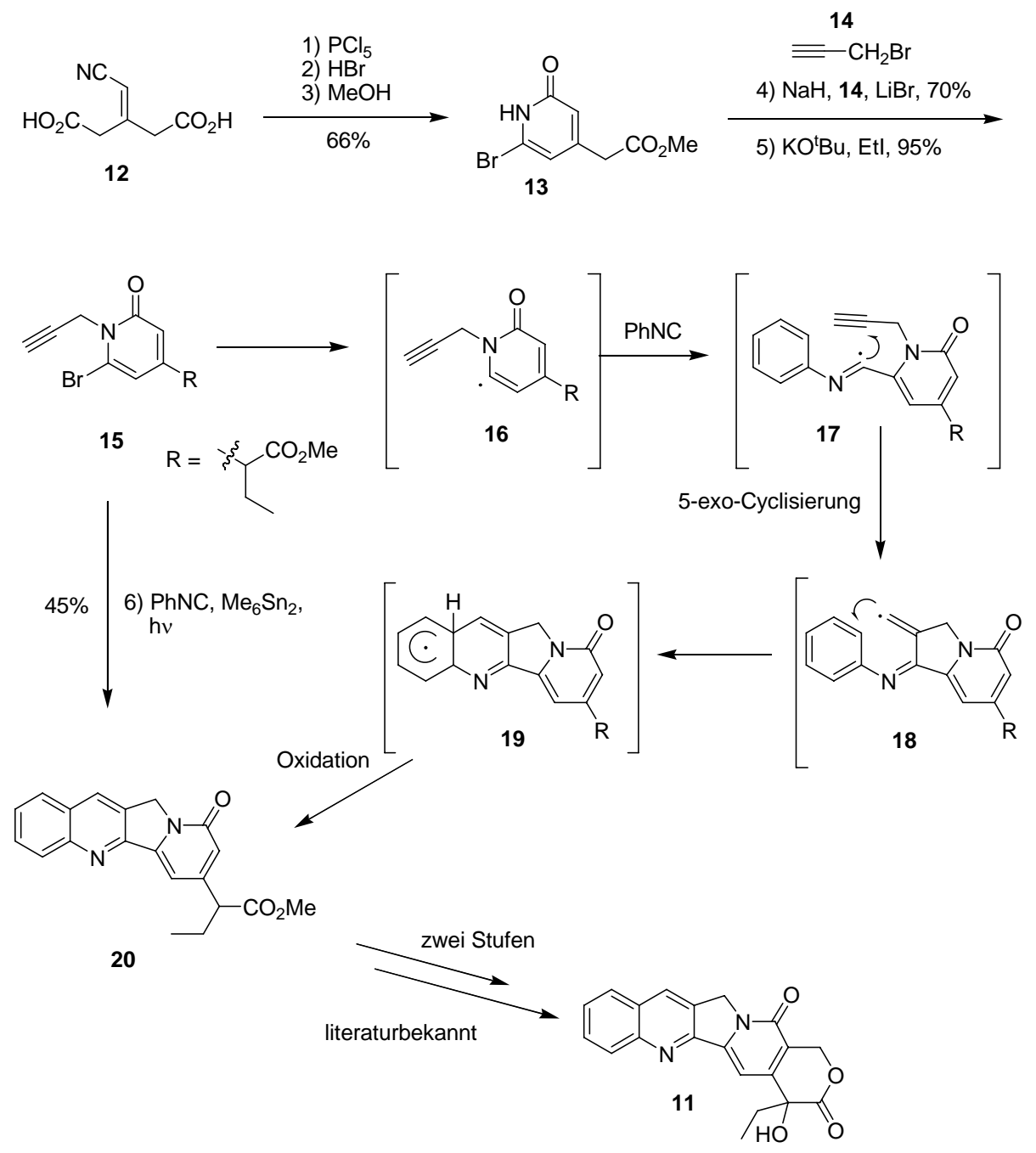

Schema 2. Formale Totalsynthese von ( \pm )-Camptothecin durch (4+1)-radikalische Annelierung

Vor kurzer Zeit gelang Z. Yao eine enatioselektive Totalsynthese von (20S)-Camptothecin. ${ }^{28}$ Als Schlüsselschritt diente ein auf eine intramolekulare aza-Diels-Alder-Reaktion basierender hoch effizienter Domino-Prozess (siehe Schema 3). Ausgehend vom literaturbekannten Pyridinderivat 21 konnte der Ester 22 über die Palladium-katalytisierte Carbonylierung und $O$-Demethylierung dargestellt werden. Die anschließende $N$-Propargylierung, Hydrolyse und Amidierung lieferten das Schlüsselfragment 23. Durch die von $\mathrm{Ph}_{3} \mathrm{PO}$ und $\mathrm{Tf}_{2} \mathrm{O}$ initiierte Amidaktivierung konnte die intramolekulare Domino-Reaktion von 23 unter milden Bedingungen durchgeführt werden. Somit wurde der Enolether 26 in 96\% Ausbeute gewonnen. Durch eine nachfolgende asymmetrische Sharpless Dihydroxylierung und LactolOxidation wurde (20S)-Camptothecin in 47\% Gesamtausbeute und 95\% ee über acht Stufen ausgehend von 21 hergestellt. 
<smiles>CCC1=COCc2c1cc(Cl)nc2OC</smiles>

21
1) $\mathrm{PdCl}_{2}\left(\mathrm{CH}_{2} \mathrm{Cl}_{2}\right) \mathrm{dppf}$

2) $\mathrm{TMSCl}, \mathrm{Nal}, 96 \%$<smiles></smiles>

3) $14, \mathrm{~K}_{2} \mathrm{CO}_{3} \mathrm{LiBr}, \mathrm{TBABr}$,

$\equiv \mathrm{CH}_{2} \mathrm{Br}$

14 kat. $\mathrm{H}_{2} \mathrm{O}, 70 \%$

4) $\mathrm{LiOH}, \mathrm{THF} / \mathrm{H}_{2} \mathrm{O}, 94 \%$

5) $(\mathrm{COCl})_{2}$, dann Anilin, $96 \%$
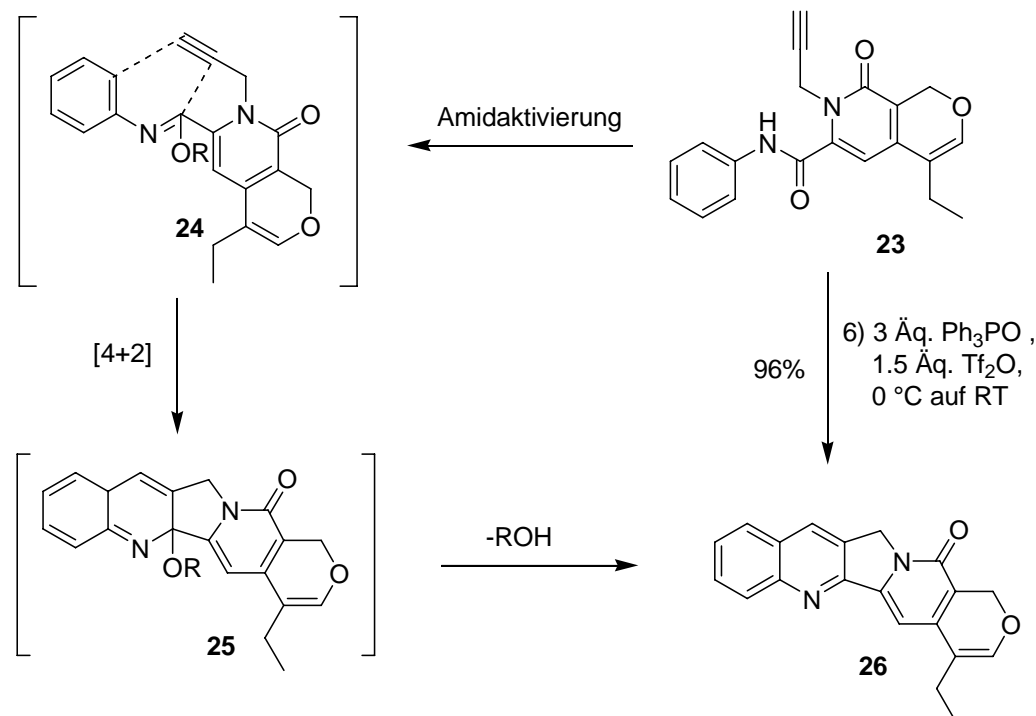

$83 \%, 95 \%$ ee

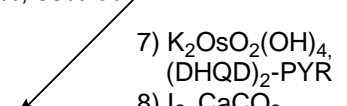

8) $\mathrm{I}_{2}, \mathrm{CaCO}_{3}$

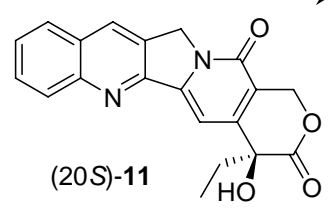

Schema 3. Enantioselektive Totalsynthese von (20S)-Camptothecin durch Domino-Reaktion 


\section{Diels-Alder-Reaktion}

\subsection{Allgemeines}

Seit ihrer Entdeckung im Jahre 1928 durch O. Diels und K. Alder ${ }^{29}$ wurde die Diels-AlderReaktion zu einer der leistungsfähigsten Methoden der organischen Synthese entwickelt. Bei der konzertierten und in den meisten Fällen regioselektiven Knüpfung von zwei Bindungen, können bis zu vier stereogene Zentren aufgebaut werden. Durch Diels-Alder-Reaktionen können durch inter- oder intramolekulare Cyclisierungen sechsgliedrige Carbo- oder Heterocyclen hergestellt werden. Die Variationsmöglichkeiten der Substrate und der Reaktionsführung sowie die große Toleranz gegenüber vielen verschiedenen Funktionalitäten führten dazu, dass die Diels-Alder-Reaktion heute eine der modernsten präparativen Methode darstellt. Sie hat große Bedeutung für die Synthese verschiedener Verbindungen, insbesondere auch von Naturstoffen. Ein eindrucksvolles Beispiel einer hoch stereoselektiven hetero-Diels-Alder-Reaktion zeigt kürzlich die Arbeitsgruppe von D. A. Evans bei der Totalsynthese von (+)-Azaspiracid-1 (siehe Schema 4). ${ }^{30}$ Unter der katalytischen Wirkung des $\mathrm{Cu}^{2+}$-Komplexes 29 konnte das Cycloaddukt 30 in der hetero-Diels-Alder-Reaktion mit hoher Diastereoselektivität und Enantioselektivität aus 27 und 28 aufgebaut werden. Über die anschließende diastereoselektive Hydrierung, nucleophile Substitution, Esterepimerisierung und Esterreduktion wurde der Aldehyd 32 mit hoher Diastereoselektivität als E-Ringfragment zur Totalsynthese bereitgestellt. 


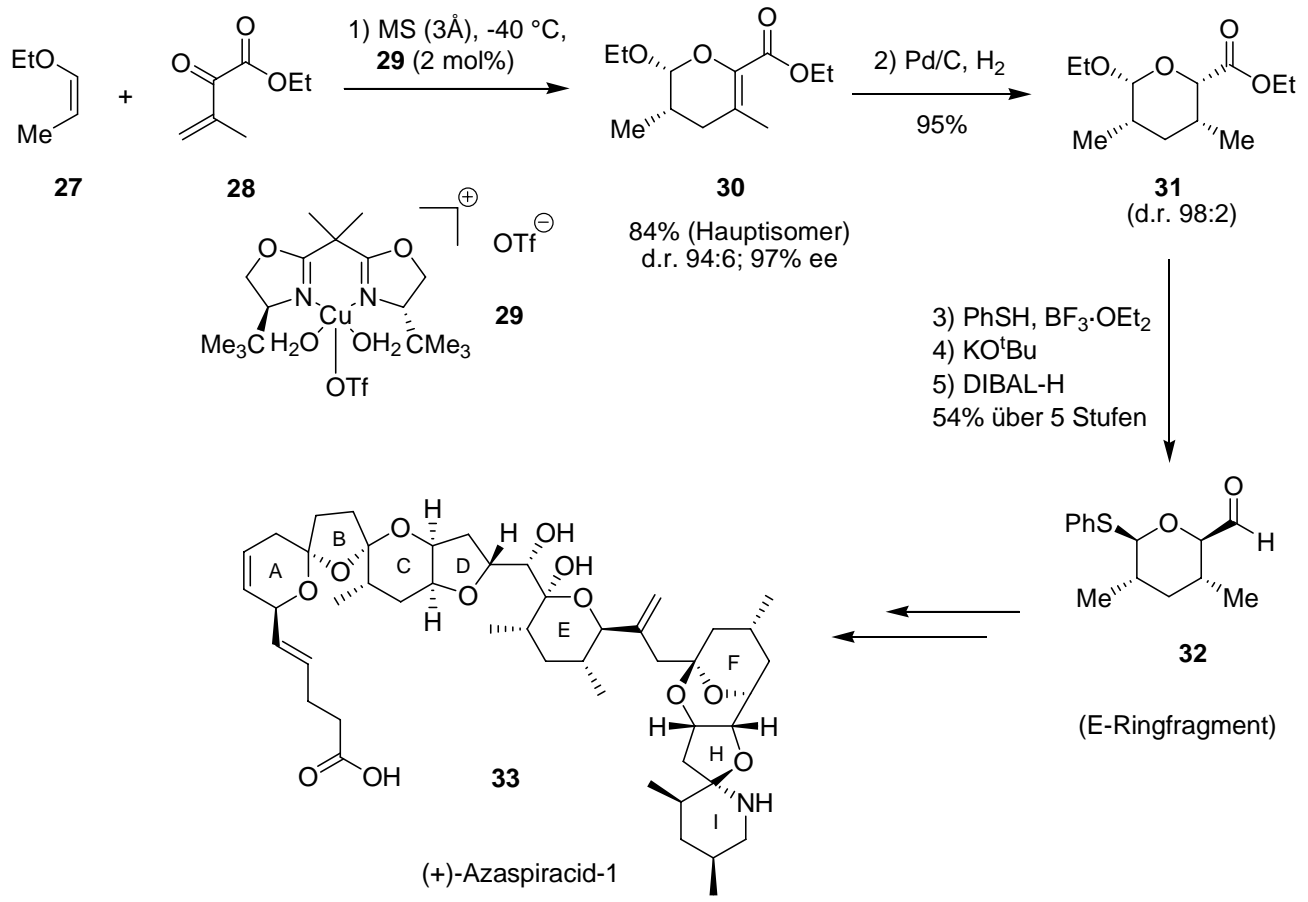

Schema 4. Stereoselektive hetero-Diels-Alder-Reaktion in der Totalsynthese von (+)-Azaspiracid-1

Eine doppelte transannulare Diels-Alder-Reaktion wurde von E. J.Sorensen zur Totalsynthese von (+)-FR182877 angewandt (siehe Schema 5). ${ }^{31}$ Unter leichter Erwärmung von 34 wurde eine Domino-Reaktion gestartet. Eine initiierende transannulare Diels-AlderReaktion und nachfolgende terminierende transannulare hetero-Diels-Alder-Reaktion konnten in einem Schritt den Pentacyclus 36 liefern. Dieser hoch ökonomische und diastereoselektive Domino-Prozess veranschaulicht das bedeutsame synthetische Potential von Diels-AlderReaktionen.
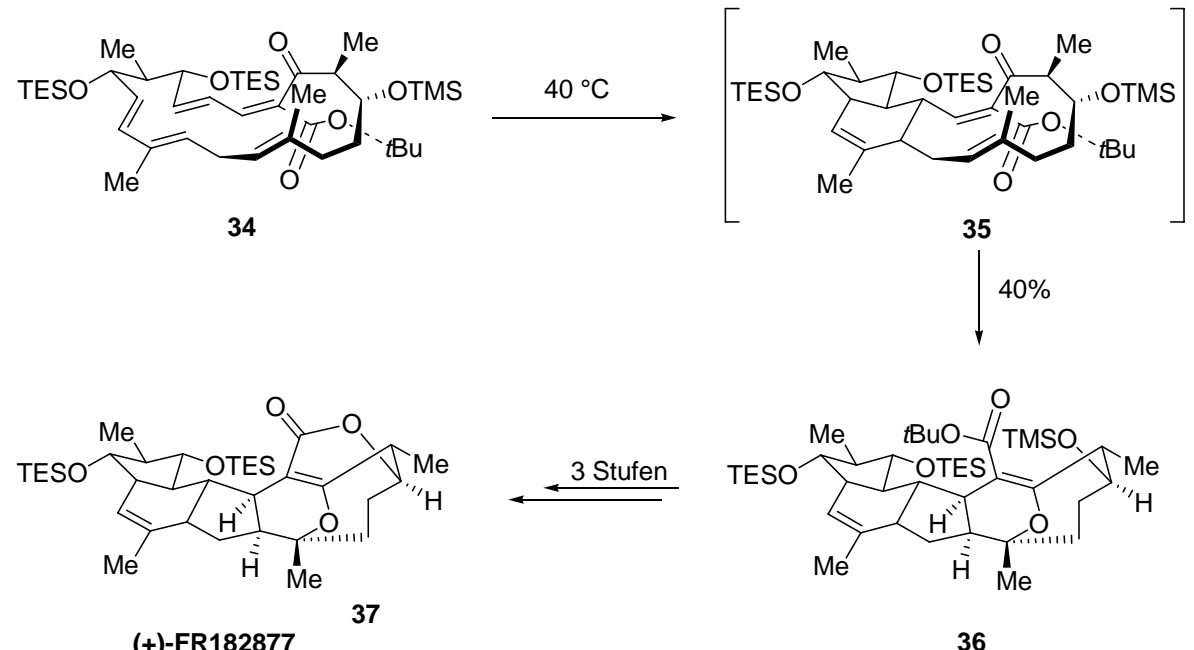

Schema 5. Doppelte transannulare Diels-Alder-Reaktion in der Totalsynthese von (+)-FR182877 


\subsection{Theorie der Diels-Alder-Reaktion}

Bei der Diels-Alder-Reaktion bildet sich ein einfach ungesättigter Sechsring in einer [4+2]-Cycloaddition. Das einfachste Beispiel ist die Reaktion von 1,3-Butadien (38) als Dien mit Ethen (39) als Dienophil zu Cyclohexen (40).

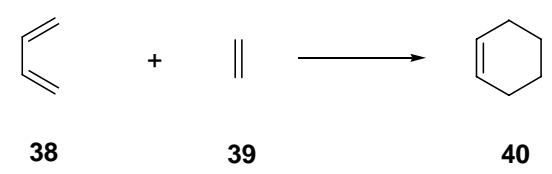

Schema 6. Diels-Alder-Reaktion

Viele mechanistische Gesetzmässigkeiten sind schon früh, zum Teil bereits von ihren Entdeckern formuliert worden. ${ }^{32}$ R. B. Woodward und R. Hoffmann haben durch die Betrachtung der Symmetrie der Molekülorbitale (MOs), die durch Linearkombination der Atomorbitale eines Moleküls gebildet werden, viele Aspekte der Diels-Alder-Reaktion aufgeklärt. ${ }^{33}$ Demnach handelt es sich bei dieser Reaktion um eine thermisch erlaubte, orbitalkontrollierte [4+2]-Cycloaddition mit supra-suprafacialer Anordnung der reagierenden $\pi$-Systeme während des gesamten Reaktionsverlaufs. Nach dem Grenzorbitalmodell, das auf die Frontier-Molecular-Orbital-Theorie von K. Fukui ${ }^{34}$ und den von K. N. Houk ${ }^{35}$ beschriebenen Begriff des Orbitalkoeffizienten zurückgeht, ist die Reaktivität der beteiligten Systeme im wesentlichen abhängig von der Energiedifferenz zwischen dem höchsten besetzten (HOMO) und dem niedrigsten unbesetzten Molekülorbital (LUMO). Damit ermöglicht das Modell eine Erklärung für die Reaktivität der Edukte bei Diels-AlderReaktionen.

Die Energie $\Delta \mathrm{E}$, mit der der Übergangszustand stabilisiert wird, kann mit Hilfe der von K. Klopmann und L. Salem aufgestellten Gleichung 1 berechnet werden:

$$
\Delta \mathrm{E}=\underbrace{-\sum_{\mathrm{ab}}\left(\mathrm{q}_{\mathrm{a}}+\mathrm{q}_{\mathrm{b}}\right) \beta_{\mathrm{ab}} \mathrm{S}_{\mathrm{ab}}+\underbrace{\frac{\mathrm{Q}_{\mathrm{k}} \mathrm{Q}_{1}}{\varepsilon \mathrm{R}_{\mathrm{kl}}}}_{\mathrm{k}<1}}_{\text {1. Term }}+\underbrace{\left.\sum_{\mathrm{r}}^{\text {bes. unbes. bes. unbes. }} \sum_{\mathrm{s}}-\sum_{\mathrm{s}} \sum_{\mathrm{r}}\right) 2 \frac{\left(\sum_{\mathrm{ab}} \mathrm{c}_{\mathrm{ra}} \mathrm{c}_{\mathrm{sb}} \beta_{\mathrm{ab}}\right)^{2}}{\mathrm{E}_{\mathrm{r}}-\mathrm{E}_{\mathrm{s}}}}_{\text {3. Term }}
$$
1. Term
2. Term
3. Term 
Hierin bedeuten:

$\mathrm{q}_{\mathrm{a}}$ und $\mathrm{q}_{\mathrm{b}} \quad$ : die Elektronenpopulationen in den Atomorbitalen $\mathrm{a}$ und $\mathrm{b}$.

$\beta_{\mathrm{ab}}$ und $\mathrm{S}_{\mathrm{ab}}$ : die Resonanz- bzw. Überlappungsintegrale der Atomorbitale $\mathrm{a}$ und $\mathrm{b}$.

$\mathrm{Q}_{\mathrm{k}}$ und $\mathrm{Q}_{1}$ : die Gesamtladung an den Atomen a und $\mathrm{b}$.

$\varepsilon \quad$ : die lokale Dielektrizitätskonstante.

$\mathrm{R}_{\mathrm{kl}} \quad$ : den Abstand zwischen den Atomen a und $\mathrm{b}$.

$\mathrm{c}_{\mathrm{ra}} \quad$ : den Koeffizienten des Atomorbitals a im Molekülorbital r.

$\mathrm{c}_{\mathrm{sb}} \quad$ : den Koeffizienten des Atomorbitals b im Molekülorbital s.

$\mathrm{E}_{\mathrm{r}}, \mathrm{E}_{\mathrm{s}} \quad$ : die Energien der Molekülorbitale $\mathrm{r}$ und $\mathrm{s}$.

Die drei verschiedenen Terme repräsentieren dabei die unterschiedlichen Arten von Wechselwirkungen der sich annähernden Reaktanden. Der erste Term, die sogenannte „closed-shell“"-Abstossung, beschreibt die antibindenden Wechselwirkungen der besetzten Molekülorbitale der reagierenden Moleküle und beinhaltet den grössten Anteil der Aktivierungsenergie der Reaktion. Im zweiten Term werden die Coulomb-Wechselwirkungen der Moleküle betrachtet, die aber nur bei ionischen oder stark polaren Molekülen von Bedeutung sind. Der dritte Term beschreibt die Wechselwirkungen aller besetzten mit allen unbesetzten Molekülorbitalen korrekter Symmetrie.

Geht man davon aus, dass bei ähnlichen oder verwandten Reaktionswegen die „closed-shell“" Abstoßung gleich ist und es sich nicht um polare oder ionische Moleküle handelt, so können die ersten beiden Terme der Gleichung 1 vernachlässigt werden. Je größer die energetischen Differenzen von Molekülorbitalen werden, desto geringer wird ihre Bedeutung für die Größe des dritten Terms. Man kann sich daher auf die Betrachtung der energetisch am dichtesten zusammenliegenden Molekülorbitale, also auf das HOMO und das LUMO, den sogenannten Grenzorbitalen, beschränken und erhält daher folgende vereinfachte Gleichung 2:

$$
\Delta \mathrm{E}=\frac{\left(\mathrm{c}_{1 \mathrm{HOMO}} \mathrm{c}_{1^{\prime} \mathrm{LUMO}} \beta_{11^{\prime}}+\mathrm{c}_{4 \mathrm{HOMO}} \mathrm{c}_{2^{\prime} \mathrm{LUMO}} \beta_{42^{\prime}}\right)^{2}}{\mathrm{E}_{\text {HOMO(Dien) }}-\mathrm{E}_{\text {LUMO(Dienophil) }}}+\frac{\left(\mathrm{c}_{1 \mathrm{LUMO}} \mathrm{c}_{1^{\prime} \mathrm{HOMO}} \beta_{11^{\prime}}+\mathrm{c}_{4 \mathrm{LUMO}} \mathrm{c}_{2^{\prime} \mathrm{HOMO}} \beta_{42^{\prime}}\right)^{2}}{\mathrm{E}_{\text {HOMO(Dienophil) }}-\mathrm{E}_{\text {LUMO(Dien) }}}
$$

Gleichung 2. Vereinfachte Klopmann-Salem-Gleichung 
Die Orbitalenergien lassen sich experimentell bzw. quantenmechanisch bestimmen. Die HOMO-Energie entspricht dem negativen Wert des ersten Ionisierungspotentials und ist durch Photoelektronenspektroskopie ermittelbar. ${ }^{36}$ Die LUMO-Energie ergibt sich aus polarographisch bestimmbaren Redoxpotentialen. ${ }^{37}$

Aus der Klopmann-Salem-Gleichung geht hervor, dass der Energiegewinn einer Reaktion umso höher ist, je geringer die Energiedifferenz zwischen dem HOMO und dem LUMO der jeweiligen Reaktanden ist. Zum Erreichen einer Reaktivitätssteigerung muss die HOMOEnergie durch elektronenliefernde Substituenten angehoben und die LUMO-Energie durch elektronenziehende Substituenten abgesenkt werden. Je nach energetischer Lage der HOMOs und LUMOs der Reaktanden kann man die Diels-Alder-Reaktion in drei verschiedene Klassen unterteilen (siehe Abbildung 4). ${ }^{38}$ Typ I wird als Diels-Alder-Reaktion mit normalen Elektronenbedarf bezeichnet. Bei diesem Typ dominiert die Wechselwirkung zwischen dem HOMO des Diens und dem LUMO des Dienophils. Elektronendonatoren im Dien und Elektronenakzeptoren im Dienophil erhöhen die Reaktivität. Die Diels-Alder-Reaktion mit neutralem Elektronenbedarf zeichnet sich durch ähnliche oder gleiche Orbitalenergien beider Reaktanden aus. Beide HOMO/LUMO-Wechselwirkungen müssen berücksichtigt werden, so dass sich die Einführung jeglicher Art von Substituenten reaktivitätssteigernd auswirkt. Bei der Diels-Alder-Reaktion mit inversen Elektronenbedarf dominiert die Wechselwirkung zwischen dem LUMO des Diens mit dem HOMO des Dienophils. Elektronenakzeptoren im Dien und Elektronendonatoren im Dienophil führen zu einer Reaktionsbeschleunigung.

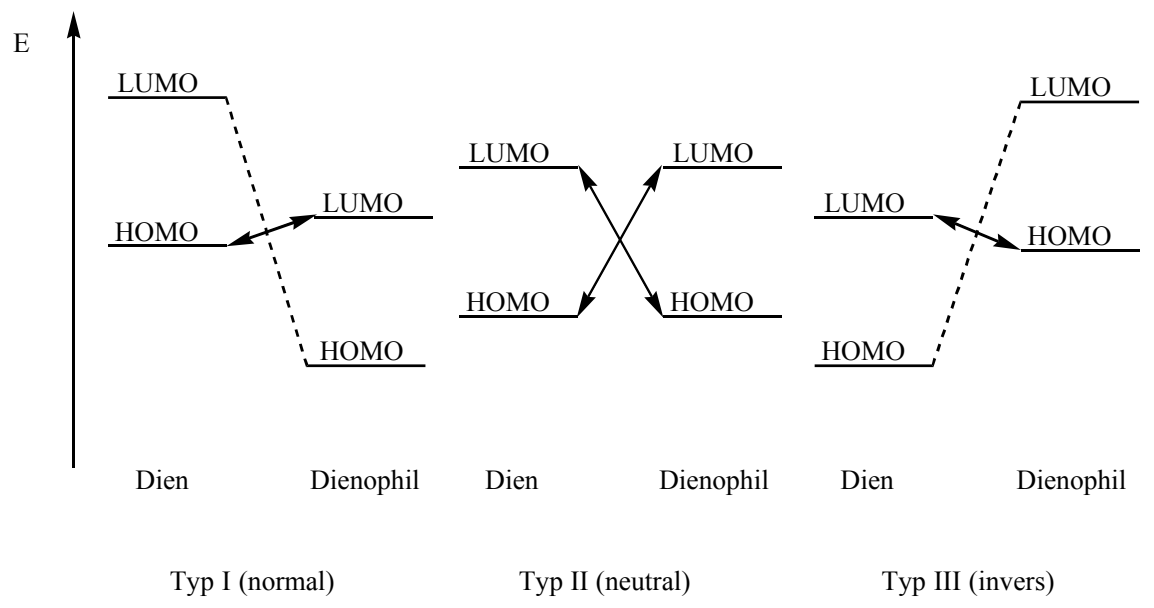


Die Einflüsse der Substituenten auf die HOMO- und LUMO-Energien und die Größe der Orbitalkoeffizienten der reaktiven Zentren von Dien und Dienophil wurden von K. N. Houk ${ }^{39}$ bestimmt. Nach ihrer Beeinflussung der energetischen Lage des HOMO und LUMO werden drei verschiedene Substituententypen unterschieden. ${ }^{40}$

Abbildung 5 zeigt die unterschiedliche Beeinflussung der HOMO/LUMO-Energien und der Orbitalkoeffizientengröße beim Dien und Dienophil in Abhängigkeit vom Substituententyp.

a) C-Substituenten: Zur Konjugation befähigte Systeme wie Phenyl- oder Vinylgruppen. Sie senken die LUMO-Energie und heben die HOMO-Energie an.

b) Z-Substituenten: Elektronenakzeptoren, z. B. Nitro-, Carbonyl- oder Cyanogruppen, senken sowohl die HOMO- als auch die LUMO-Energie.

c) X-Substituenten: Elektronendonatoren, wie Amino-, Alkyl- oder Alkoxygruppen, heben die HOMO- und LUMO-Energie an.
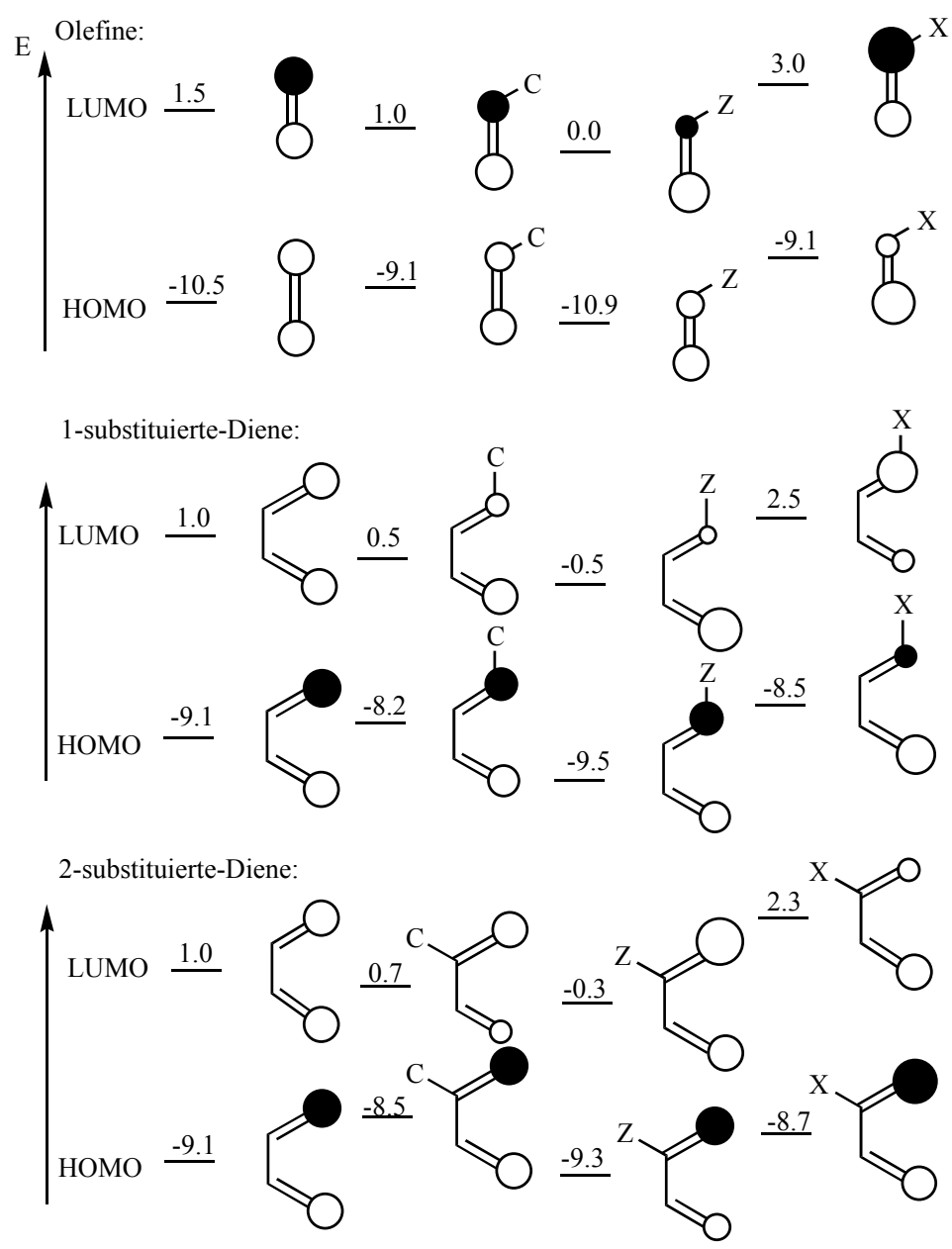

Abbildung 5. Einfluss unterschiedlicher Substituenten 
Die Orbitalkoeffizienten haben einen nicht unerheblichen Anteil an der Regioselektivität und Reaktivität einer Diels-Alder-Reaktion. Eine bindende Überlappung zweier großer Orbitalkoeffizienten ist gegenüber einer Überlappung eines kleinen mit einem großen Orbitalkoeffizienten bevorzugt.

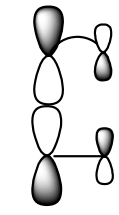

bevorzugt

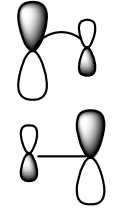

nicht bevorzugt

Abbildung 6. Überlappung von Grenzorbitalen

Der endo-Angriff des Dienophils auf das Dien zum thermodynamisch instabileren Produkt ist in der Regel bevorzugt, da der Übergangszustand aus sterischen Gründen, aufgrund von Lösungsmitteleffekten und eventuell auftretenden „Wasserstoffbrücken-Bindungen“ stabilisiert wird. ${ }^{41}$ Eine sekundäre Überlappung, wie oftmals angenommen, kann allenfalls eine marginale Stabilisierung hervorrufen. Außerdem kann die Reaktionsgeschwindigkeit unter Verwendung von Lewissäuren gesteigert und gleichzeitig eine höhere Regio- und Stereoselektivität bei der Bildung des Cycloadduktes erreicht werden.

Der Ablauf und die Richtung der Diels-Alder-Reaktion lassen sich anhand verschiedener, empirisch gefundener Gesetzmäßigkeiten vorhersagen:

a) Das cis-Prinzip: Die relative Konfiguration der Substituenten am Dien und Dienophil bleibt auch im Cycloaddukt erhalten.

b) Die Alder-Regel: Stark unterschiedliche elektronische Eigenschaften von Dien und Dienophil beschleunigen die Reaktion.

c) Die endo-Regel: Bei Additionen an cyclische Diene entsteht häufig das thermodynamisch instabilere endo-Produkt im Überschuß.

d) Die ortho-Regel: Unsymmetrisch substituierte Edukte vereinigen sich regioselektiv zum ortho-Cycloaddukt. 


\section{Domino-Reaktion}

\subsection{Konzept der Domino-Reaktion}

Die Zukunft der organischen Synthese liegt u. a. in der Entwicklung neuer effizienter Methoden, die den Aufbau komplexer Moleküle in wenigen Schritten ausgehend von einfachen Substraten erlauben. Dies ist die Stärke von Domino-Reaktionen, die mehrere bindungsbildende Schritte unter identischen oder nahezu identischen Reaktionsbedingungen in einem Prozeß kombinieren und sich häufig durch große Eleganz, hohe Stereoselektivität und einfache Reaktionsführung auszeichnen. ${ }^{42}$

Die Entwicklung derartiger Synthesemethoden bietet sowohl ökonomische als auch ökologische Vorteile: Die Menge unerwünschter Abfallprodukte wird eingeschränkt, die Zahl der Aufarbeitungsschritte und damit die benötigte Lösungs- und Elutionsmittelmenge beträchtlich verringert. Daraus erwächst sowohl ökologischer als auch ökonomischer Nutzen. ${ }^{43}$

\subsection{Domino-Reaktionen in der organischen Synthese}

Mit einer Domino-Wacker-Heck-Reaktion gelang es L. F. Tietze, das Chromangerüst 47 des $\alpha$-Tocopherols (48) enantioselektiv aufzubauen und gleichzeitig die Seitenkette einzuführen (siehe Schema 6). ${ }^{44}$ Die Sequenz beginnt wahrscheinlich mit der enantiofacialen Koordination des Pd(II)-Komplexes an das 1,1-disubstituierte Alken 41 unter Bildung des Zwischenprodukts 43. Eine intramolekulare Cyclisierung mit der freien Phenolfunktion erzeugt die $\delta$ Alkyl-Pd(II)-Spezies 44, welche aufgrund eines fehlenden $\beta$-Wasserstoffatoms keine Eliminierung eingehen konnte. Stattdessen schließt sich eine intermolekulare Heck-Reaktion mit Methylacrylat (45) an. 


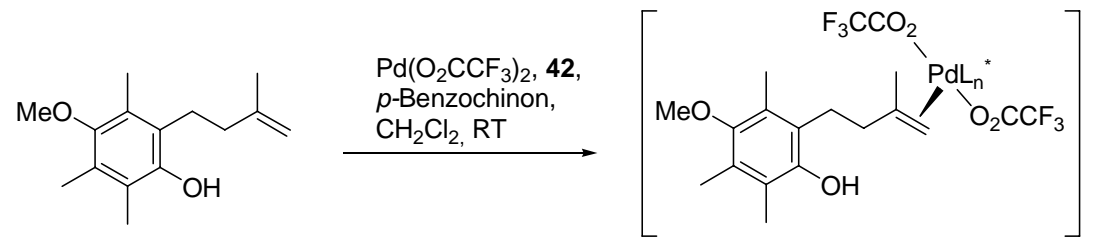

41

43

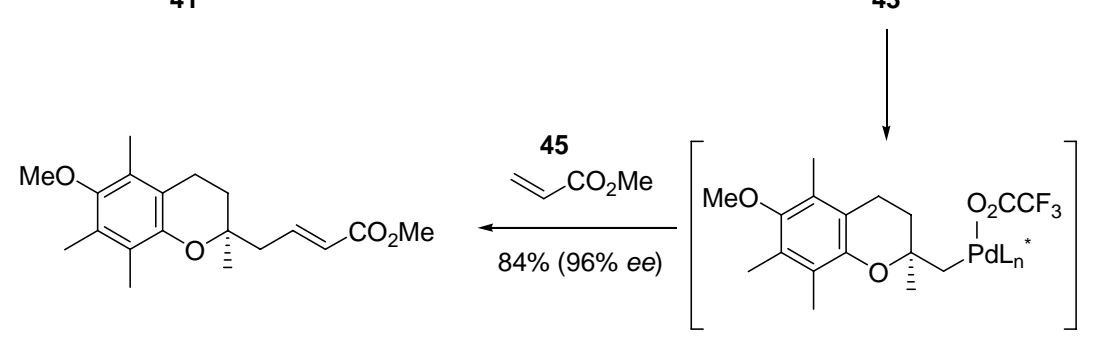

47

44
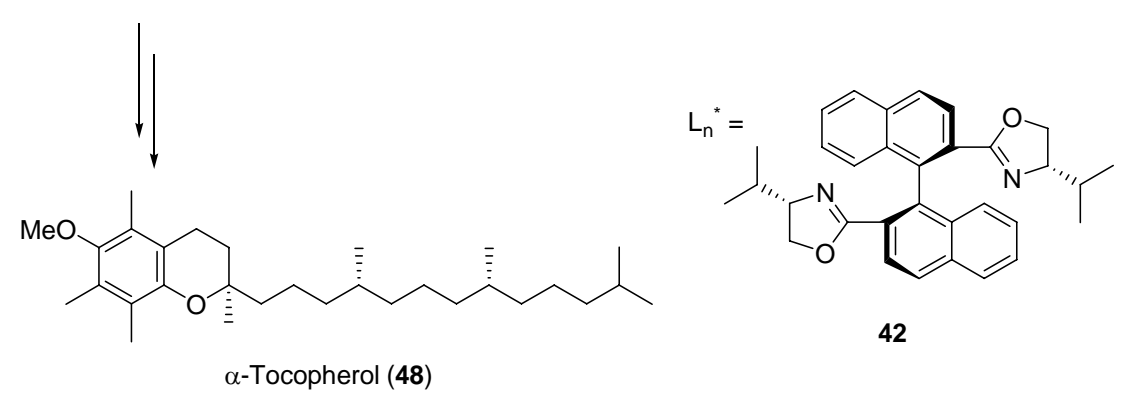

42

Schema 7. Palladium-katalytisierte enantioselektive Domino-Wacker-Heck-Reaktion

Ein ebenfalls effizienter und eleganter Zugang zum Indolalkaloid Hirsutin wurde von L. F. Tietze über eine Domino-Reaktion, in diesem Fall unter Verwendung einer DominoKnoevenagel-hetero-Diels-Alder-Reaktion entwickelt (Schema 7). ${ }^{45}$ Die KnoevenagelKondensation des enantiomerenreinen Aldehyds 49 mit Meldrum-Säure (46) lieferte das 1,3Oxabutadien 51, welches sofort eine hetero-Diels-Alder Reaktion mit dem Enolether 50 einging. Dies geschah mit einer ausgezeichneten 1,3-Induktion von $>25: 1$. In einem EintopfProzess wurde das Rohprodukt mit Methanol in Gegenwart von Kaliumcarbonat versetzt und mit Palladium auf Kohle als Katalysator hydriert. Der dabei entstehende Aldehyd reagierte mit dem freigesetzten Amin zu einem Enamin, das stereoselektiv zu 53 hydriert werden konnte. Nach Abspaltung der Boc-Schutzgruppe, Kondensation mit Ameisensäuremethylester und Behandlung mit Diazomethan konnte enantiomerenreines Hirsutin 54 erhalten werden. Aufbauend auf der Domino-Knoevenagel-hetero-Diels-Alder-Sequenz wurde von L. F. Tietze et al. das Konzept der stereochemischen Kombinatorik entwickelt, das u. a. den einfachen Aufbau einer Bibliothek von 12 Stereoisomeren des Ipecacuanha-Alkaloids Emetin ermöglichte. $^{46}$ 


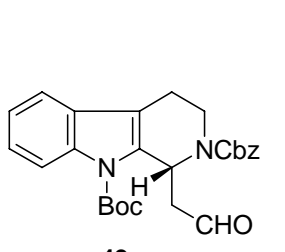

49

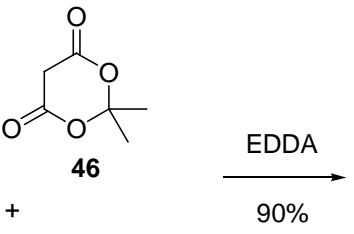

$\overbrace{}^{n} \mathrm{OPMB}$

50<smiles>CC[C@H]1CN2CCc3c(n(C(=O)c4ccccc4)c4ccccc34)[C@@]2(CCC(C)=O)C1</smiles>

53

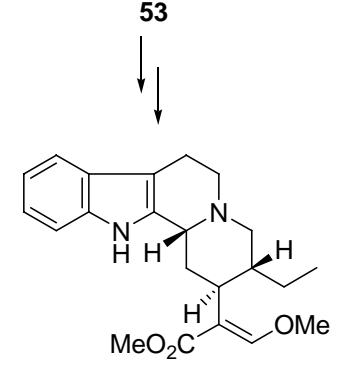

54 Hirsutin<smiles>CCCCCC[C@H]1c2c(c3ccccc3n2C(=O)c2ccccc2)CCN1C(=O)[O-]</smiles>

51

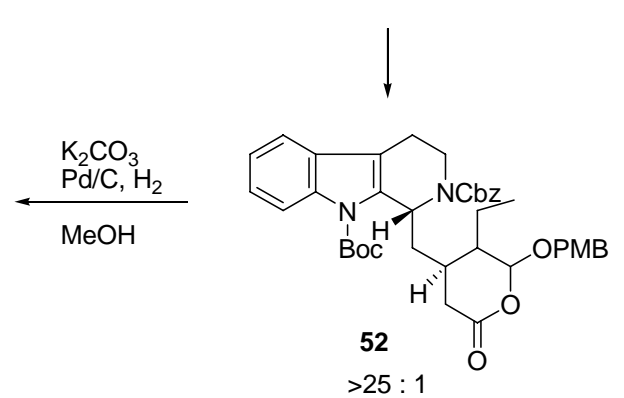

Schema 8. Substrat-basierte stereoselektive Domino-Knoevenagel-hetero-Diels-Alder-Reaktion

Eine organo-katalytisierte asymmetrische Domino-Knoevenagel-hetero-Diels-Alder-Reaktion wurde von F. Barbas III beschrieben. ${ }^{47}$ In einer Amin(58)-katalytisierten Domino-Reaktion konnten ausgehend von verschiedenen Enonen 55 und Aldehyden 56 in einem Schritt mehrere hoch substituierte Spiro[5,5]undecan-1,5,9-trione 57 in $80-95 \%$ Ausbeute und $84-99 \%$ ee dargestellt werden. 


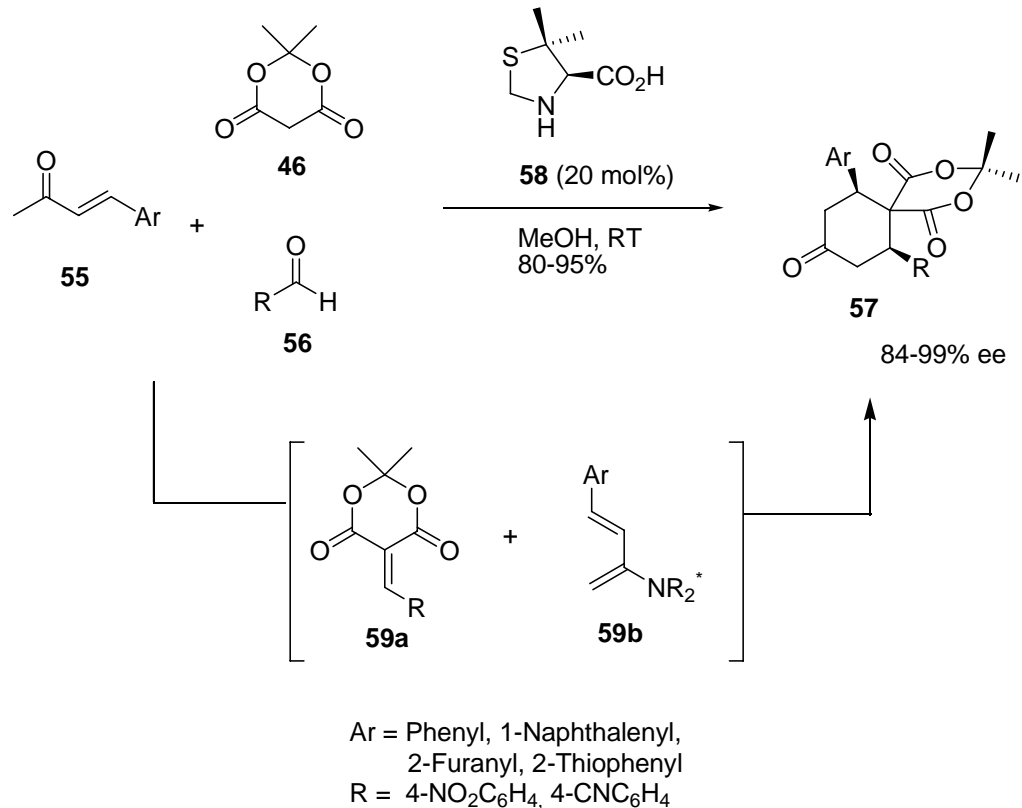

Schema 9. Organo-katalytisierte asymmetrische Domino-Knoevenagel-hetero-Diels-Alder-Reaktion 


\section{AUfGabenstellung}

\section{Zielsetzung der Arbeit}

Aufgrund seiner Bedeutung für die Krebstherapie ist Camptothecin attraktives Syntheseziel. Heute sind insgesamt ca. 30 Publikationen über seine Totalsynthese bekannt. Es fehlt aber noch ein effizienter Ansatz, welcher Camptothecin in großen Menge herzustellen ermöglicht. Domino-Reaktionen mit dem Vorteil, aus einfachen Substraten in wenigen Schritten komplexe Strukturen aufzubauen, gelten als richtungsweisende Lösung. Neben der DominoKnoevenagel-hetero-Diels-Alder-Reaktion ${ }^{9}$ sind in der Arbeitsgruppe von L. F. Tietze weitere wertvolle Transformationen wie die Domino-Knoevenagel-En-Reaktion, ${ }^{48}$ Domino-PictetSpengler-En-Reaktion ${ }^{49}$ und Domino-Wacker-Heck-Reaktion ${ }^{44}$ entwickelt. Sie sind schon erfolgreich in der Synthese von Terpenen, Steroiden, Heterocyclen, Alkaloiden und Vitamin E eingesetzt worden.

In Anlehnung an die Arbeiten von N. Rackelmann ${ }^{50}$ sollte mit Hilfe eines DominoKnoevenagel-hetero-Diels-Alder-Prozesses und einer anschließenden Domino-Reaktion bestehend aus Hydrogenolyse und intramolekularer Lactamcyclisierung das Zielmolekül Camptothecin synthetisiert werden. Hierbei sollte auch der enantioselektive Aufbau des Stereozentrums (20S) untersucht werden, da nur das S-Enantiomer des Alkaloids bzw. seiner Derivate biologisch interessant ist. 


\section{Planung der Arbeit}

Retrosynthetisch lässt sich der Naturstoff auf das Indolizinochinolin Grundgerüst 61 bzw. 62 zurückführen, das durch zwei aufeinanderfolgende Dominoprozesse aus einem Aldehyd 60 und Dienophilen 63, 64 oder 65 zugänglich sein sollte. Die Methylestergruppe in 62 kann durch einen methanolyse-modifizierten Dominoprozess eingeführt werden.

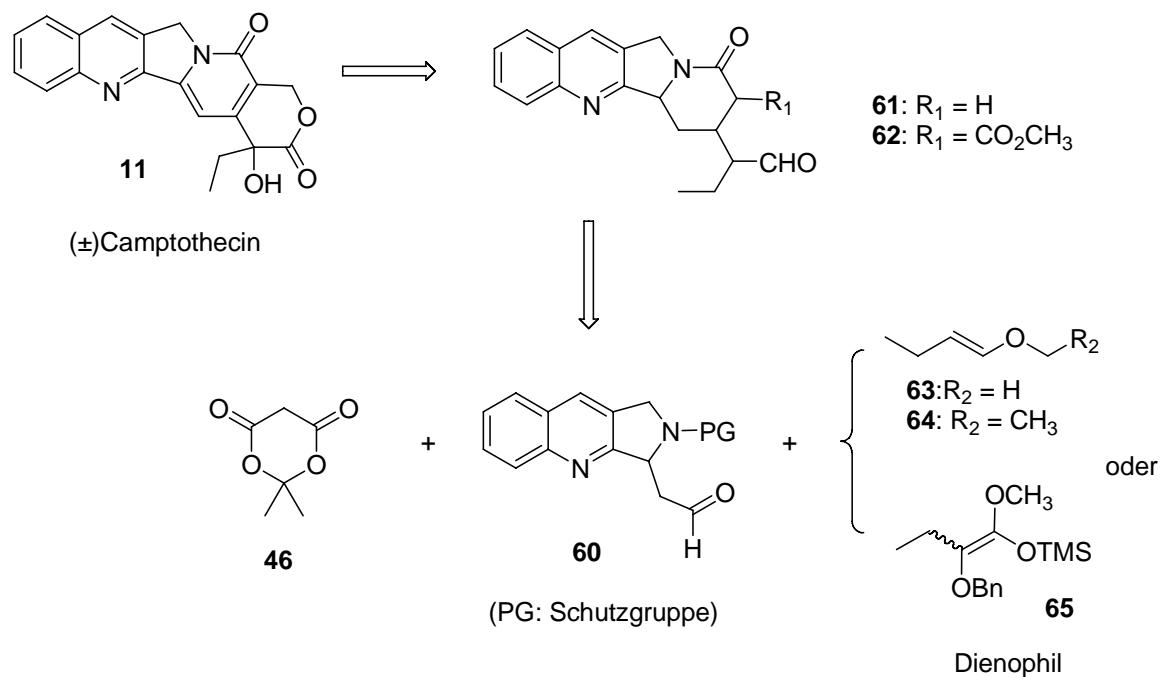

Schema 10. Retrosynthese von ( \pm -)-Camptothecin

Zur effizienten Totalsynthese von Camptothecin ergeben sich damit die folgenden Aufgaben:

- Synthese von Dienophilen 63-65

- Synthese von Aldehyden des Typs 60 über möglichst kurzen Weg sowie Optimierung der einzelnen Synthesestufen

- Durchführung der Domino-Knoevenagel-hetero-Diels-Alder Reaktionen und der methanolyse-modifizierten Transformation

- Synthese von Camptothecin

- Untersuchungen zur enantioselektiven Synthese von (20S)-Campothecin 


\section{DARSTELLUNG DER ERGEBNISSE}

Im Rahmen der Totalsynthese von Camptothecin war es zunächst erforderlich, die Substrate zur Durchführung der Domino-Knoevenagel-hetero-Diels-Alder-Reaktionen darzustellen.

\section{Synthese von Dienophilen}

\subsection{Untersuchung zur Synthese des Enolethers 63}

Der Enolether 63 sollte zuerst durch säurekatalytische Eliminierung aus dem Acetal 66 dargestellt werden. Die Acetalbildung des Butyraldehyds (67) mit p-TSA ${ }^{51}$ und Methylorthoformiat gab zwar das Acetal 66 in 53\% Ausbeute, allerdings bildeten sich bei der Reaktion Nebenprodukte, welche sich destillativ nicht entfernen ließen. Die Umsetzung mit Calciumchlorid als Trockenmittel und Katalysator lieferte das Acetal in ähnlicher Ausbeute aber sehr reiner Form. Letztlich ließ sich die Ausbeute unter katalytischer Wirkung von $\mathrm{RuCl}_{3}{ }^{52}$ auf $68 \%$ erhöhen.

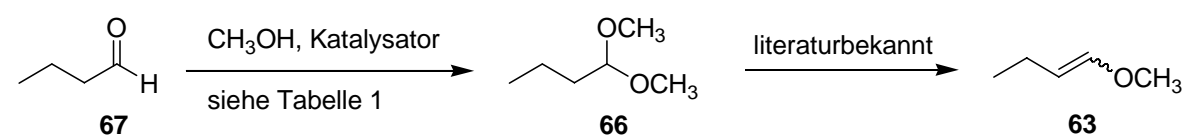

Schema 11. Synthese von 66

\begin{tabular}{|c|c|c|}
\hline Eintrag & Reaktionsbedingungen & Ausbeute \\
\hline 1 & $\begin{array}{l}1.0 \text { Äq. 67, } 3.0 \text { Äq. Methanol, } 1.2 \text { Äq. Methylorthoformiat, } \\
0.022 \mathrm{~mol} \% \text { p-TSA } \cdot \mathrm{H}_{2} \mathrm{O}, 0{ }^{\circ} \mathrm{C} \text { auf RT, } 20 \mathrm{~h}\end{array}$ & $53 \%$ \\
\hline 2 & $\begin{array}{l}1.0 \text { Äq. 67, } 2.9 \text { Äq. Methanol, } 1.0 \text { Äq. } \mathrm{CaCl}_{2}, \mathrm{RT}, 3 \text { h; dann } \\
\text { über Nacht stehen }\end{array}$ & $54 \%$ \\
\hline 3 & $\begin{array}{l}1.0 \text { Äq. 67, } 5.0 \text { Äq. Methanol, } 0.10 \mathrm{~mol} \% \mathrm{RuCl}_{3} \times \mathrm{xH}_{2} \mathrm{O}, 0{ }^{\circ} \mathrm{C} \\
\text { auf RT, } 17 \mathrm{~h}\end{array}$ & $68 \%$ \\
\hline
\end{tabular}

Tabelle 1. Synthese von 66 unter Einsatz verschiedener Katalysatoren 
Für die anschließende Eliminierung wurden p-TSA, Phosphonsäure und aq. Phosphorsäure verwendet. Nach Destillation konnte kein Produkt detektiert und nur das Edukt zurückgewonnen werden.

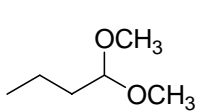

66

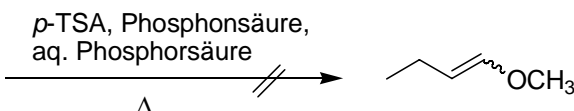

63

Schema 12. Untersuchung der Synthese von 63 durch Eliminierung

Als alternative Methode diente die Wittg-Reaktion ausgehend von Propionaldehyd (68) mit den Methoxymethyltriphenylphosphoniumsalzen MMTPBr oder MMTPCl. Trotz umfangreichen Versuchen ließ sich keine Produktbildung unter Verwendung von $\mathrm{NaNH}_{2}$ in Tetrahydrofuran oder Diethylether bestätigen. Die Bildung des charakteristisch roten Ylids ${ }^{53}$ war entsprechend auch nicht zu beobachten. In einzelnen Fällen konnte nur der entsprechende Phosphinoxid 69 in betrachtlichen Mengen (> 60\%) isoliert werden. Sogar das Instant-Ylid, ${ }^{54}$ ein kommerziell erhältliches Gemisch von $\mathrm{MMTPBr}$ und $\mathrm{NaNH}_{2}$ (1:1) konnte nicht das gewünschte Produkt unter den Bedingungen darstellen. Ein Wechsel der Base zu KotBu konnte zwar das Ylid von MMTPCl liefern, die Isolierung des Produktes erwies sich aber als problematisch. Nach üblicher Aufarbeitung und sorgfältiger Entfernung des Lösungsmittels ließ sich kein Signal des Produktes im ${ }^{1} \mathrm{H}-\mathrm{NMR}$ des Rohproduktes beobachten.

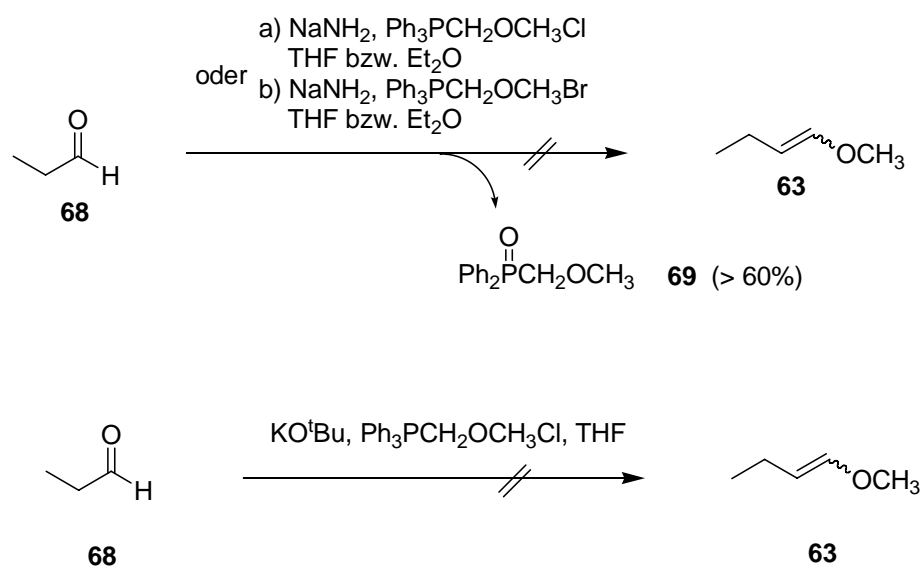

Schema 13. Untersuchung der Synthese von 63 durch Wittig-Reaktion 


\subsection{Synthese des Enolethers 64}

Aufgrund der Schwierigkeit, 63 zu synthetisieren, wurde die Synthese von 64 untersucht. Dazu wurde zuerst das Acetal $\mathbf{7 0}$ unter katalytischer Wirkung von $\mathrm{RuCl}_{3}$ unter milden Bedingungen in $47 \%$ Ausbeute herstellt. Eine Verbesserung wurde erzielt, indem die Acetalisierung bei erhöhter Temperatur unter Verwendung von Amberlyst-15 und Ethylorthoformiat durchgeführt wurde. Das Acetal konnte dann in 67\% Ausbeute nach destillativer Reinigung dargestellt werden. Für die anschließende Herstellung des Enolethers 64 wurde Sulfanilsäure ${ }^{55}$ verwendet. Im Gegensatz zum dimethyl-substituierten Acetal 66 konnte das diethyl-substituierte Acetal 70 in guter Ausbeute zu 64 eliminiert werden.

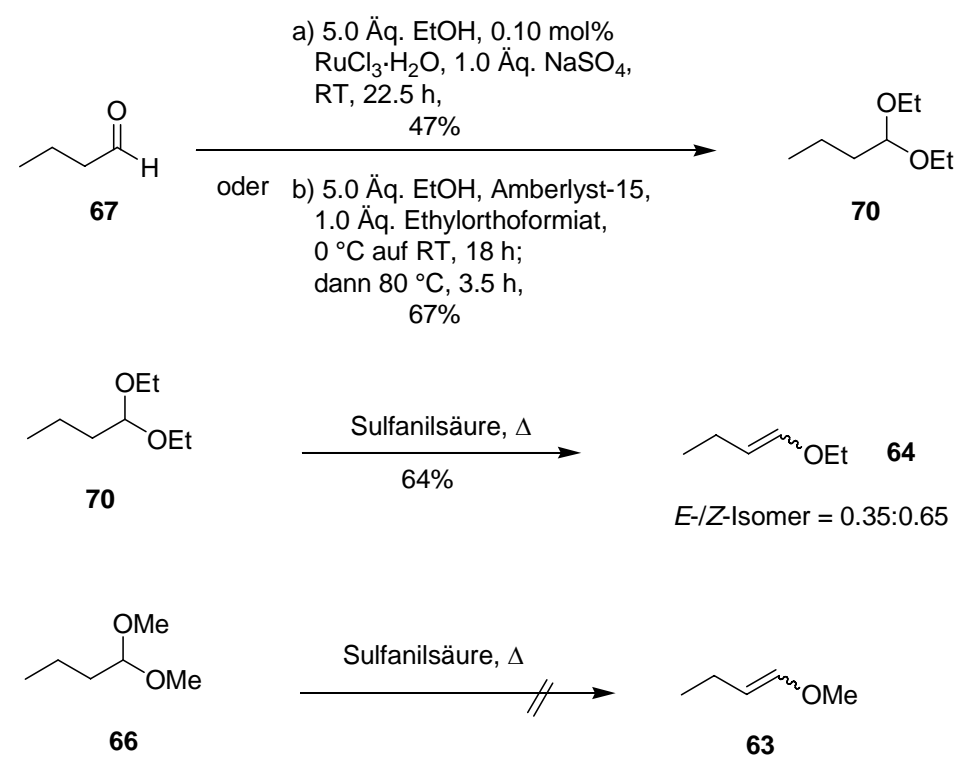

Schema 14. Synthese von 64

\subsection{Untersuchung zur Synthese des Enolethers 65}

Zur Synthese von 65 wurde zuerst die kommerziell erhältliche $\alpha$-Ketosäure 72 in Methanol mit katalytischen Mengen Chlortrimethylsilan ${ }^{56}$ umgesetzt. Die Veresterung lieferte den Ester 73 ohne weitere Aufreinigung in 87\% Ausbeute. Die anschließende selektive Reduktion der Ketofunktion konnte mit $\mathrm{NaBH}_{4}$ in Methanol erreicht werden. Der Alkohol 74 wurde in $76 \%$ Ausbeute erhalten. Die geringere Ausbeute ließ sich auf die Bildung der doppelt reduzierten Spezies zurückführen, allerdings sollte sich dieses Nebenprodukt durch wässrige Aufarbeitung leicht entfernen lassen. Die anschließende $O$-Benzylierung ließ sich nicht durch konventionelle nucleophile Substitution realisieren. Der Wechsel zu DMF und TBAI konnte keine Verbesserung bewirken. 


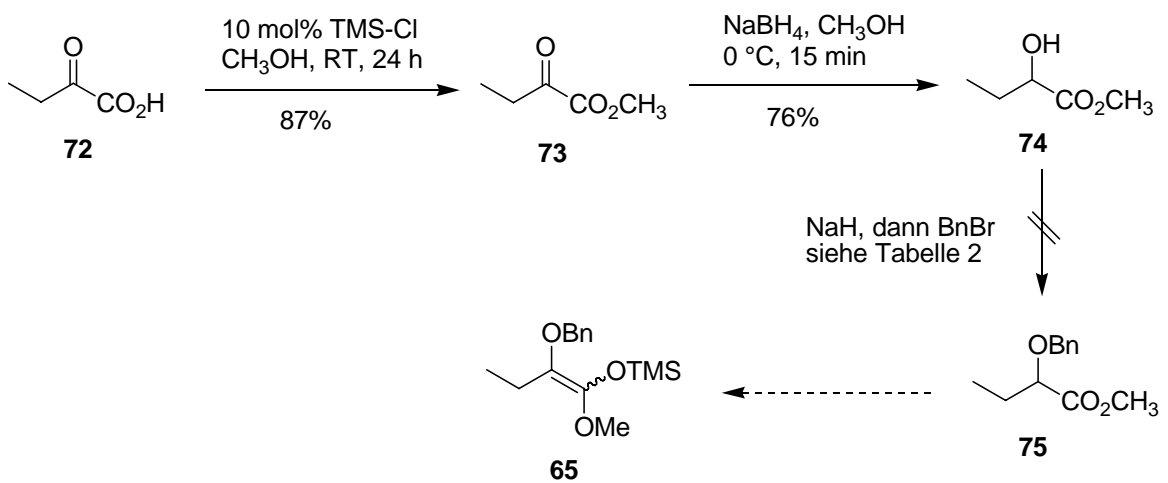

Schema 15. Untersuchung der Synthese von 65 ausgehend von $\alpha-$ Ketosäure 72

\begin{tabular}{|c|c|c|}
\hline Eintrag & Reaktionsbedingungen & Ausbeute \\
\hline 1 & $\begin{array}{l}1.0 \text { Äq. 74, } 1.1 \text { Äq. NaH, THF, } 0^{\circ} \mathrm{C} \text { auf RT, } 2.5 \text { h; dann } 1.0 \\
\text { Äq. BnBr, RT, } 23 \text { h }\end{array}$ & - \\
\hline 2 & $\begin{array}{l}1.0 \text { Äq. 74, } 1.1 \text { Äq. NaH, DMF, } 0{ }^{\circ} \mathrm{C} \text { auf RT, } 1.5 \text { h; dann } 1.0 \\
\text { Äq. BnBr, RT, } 27 \text { h }\end{array}$ & - \\
\hline 3 & $\begin{array}{l}1.0 \text { Äq. 74, } 1.0 \text { Äq. BnBr, } 1.1 \text { Äq. } \mathrm{NaH}, 5.0 \mathrm{~mol} \% \text { TBAI, } \\
\text { THF, } 0{ }^{\circ} \mathrm{C} \text { auf RT, } 22 \mathrm{~h} \text {; dann } 40^{\circ} \mathrm{C}, 2 \mathrm{~h}\end{array}$ & - \\
\hline
\end{tabular}

Tabelle 2. Untersuchung der O-Benzylierung von $\mathbf{7 4}$

Ausgehend von $\alpha$-Brommethylester 76 konnte mit Natriumbenzylalkoxid zwar $\alpha-$ Benzyloxyester 75 NMR-spektroskopisch detektiert werden, allerdings begünstigten die Reaktionsbedingungen hauptsächlich die Bildung der Eliminierungs- bzw. Umesterungsprodukte, weswegen für die weitere Umsetzung andere Bedingungen verwendet werden mussten.

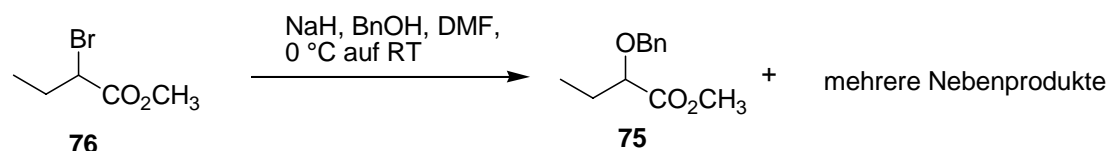

Schema 16. Versuch zur Synthese von $\mathbf{7 5}$ aus 76

Anschließend wurde die Synthese von 65 ausgehend von $\alpha$-Brombuttersäure (77) untersucht. Die nucleophile Substitution mit Natriumbenzylalkoxid und anschließende Verersterung mit Methanol unter Chortrimethylsilan-Katalyse lieferten den $\alpha$-Benzyloxyester 75 in einer Ausbeute von $77 \%$ über zwei Stufen. Die weitere Synthese des Enolethers 65 über 
Enolatbildung blieb aber erfolglos. Obwohl ähnliche Umsetzungen mit LDA literaturbekannt sind, ${ }^{57}$ konnte keine Reaktion unter den untersuchten Bedingungen beobachtet werden. Eine Massenanalyse des Rohproduktes gab keinen Hinweis auf das Vorliegen von 65.

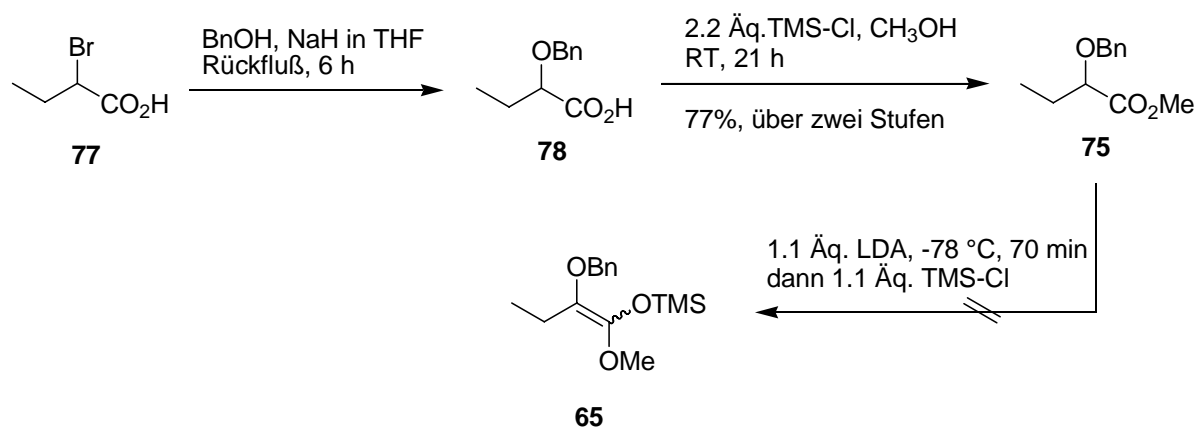

Schema 17. Untersuchung der Synthese von 65 ausgehend von $\alpha$-Brombuttersäure (77) 


\section{Synthese von Aldehyden}

Basierend auf den Arbeiten von S. P. Chavan $^{58}$ sollten verschiedene Aldehyde des Typs 60 ausgehend von kommerziell erhältlichem Acetanilid (79) über die folgende Synthesewege als Vorläufer der Domino-Reaktionen dargestellt werden.

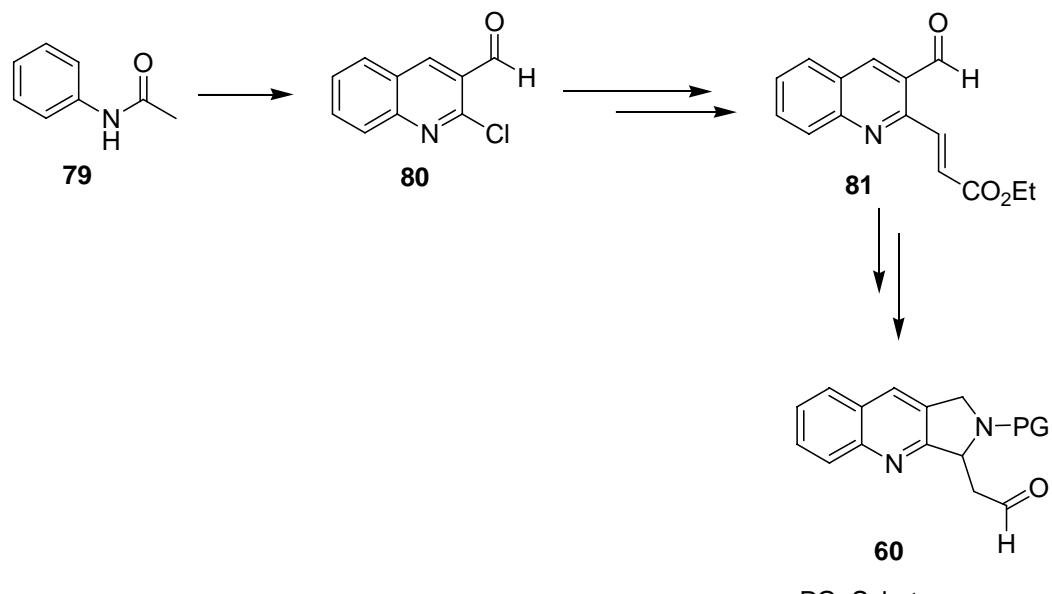

PG: Schutzgruppe

Schema 18. Vorgeschlagene Wege zur Synthese von Aldehyden

\subsection{Synthese des Chinolin-Gerüstes}

Der Meth-Cohn-Aldehyd 80 ließ sich durch Vilsmeier-Haack Cylisierung ${ }^{59}$ von Acetanilid (79) darstellen. Nach 16.5 Stunden bei $75{ }^{\circ} \mathrm{C}$ konnte 80 in 64\% Ausbeute gewonnen werden. Der genaue Reaktionsverlauf lässt sich über den folgenden Mechanismus erklären (siehe Schema 20). Unter der Wirkung von Phosphoroxychlorid wird Acetanilid (79) in das Enamin (84) umgewandelt, welches anschließend auf das in situ gebildete Elektrophil (82, VilsmeierReagenz) angreift. Weitere Eliminierug, Addition und intramolekulare aromatische Cyclisierug liefern den Aldehyd 80 .

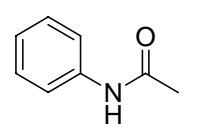

79

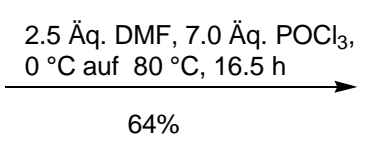

$64 \%$

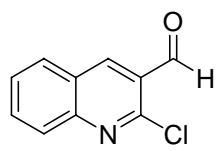

80

Schema 19. Synthese von 80 durch Vilsmeier-Haack Cylisierung 
Vilsmeier-Haack Cyclisierung
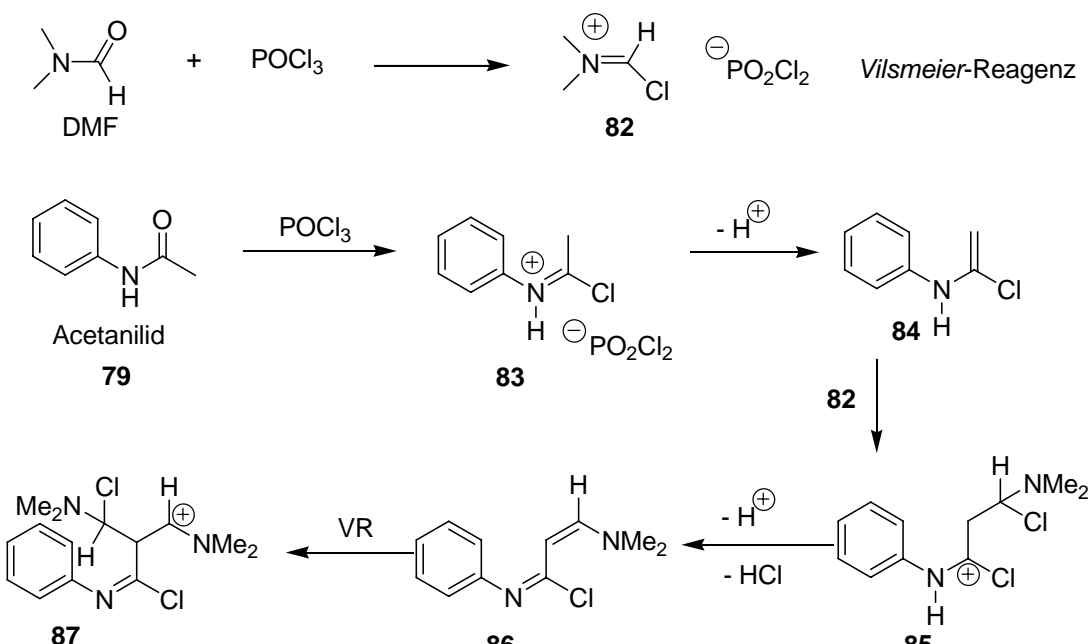
$-\mathrm{HCl}$<smiles>C=C(/C(=N\c1ccccc1)C(Cl)=NC)N(C)C</smiles>

88
86

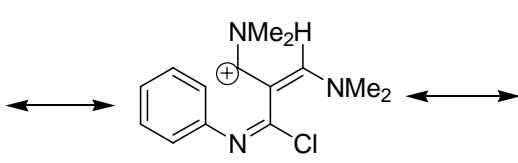

89

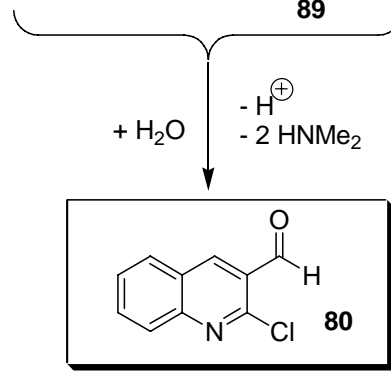

85<smiles></smiles>

90

Schema 20. Mechanismus für Vilsmeier-Haack Cylisierung

Da Arylchlorid prinzipiell kein gutes Substrat für eine weitere Heck-Kupplung ist, wurde anschließend eine Iodierung durchgeführt. Durch Umsetzung von 80 mit Natriumiod und wäßriger $\mathrm{HCl}$ in Acetonitril unter Rückfluss ${ }^{60}$ konnte das Iodchinolin 91 in hoher Ausbeute von $84-89 \%$ erhalten werden. Das Produkt enthielt 3-10 mol\% des Eduktes. Die vollständige Entfernung des Eduktes aus dem Gemisch konnte weder durch Chromatographie noch Umkristallisation erreicht werden. Verwendung von einem höheren Überschuß von Natriumiod führte nicht zu einer Verbesserung der Reaktion. Anschließend wurde versucht, durch Bildung des Chinoliniumsalzes 92 einen Chlor-Iodaustausch zu bewirken, da sich diese Reaktion bei Aminsalzen leicht vollzieht. ${ }^{61}$ Aber die Salzbildung mit trocknem $\mathrm{HCl}$ blieb erfolglos, wie sowohl durch ${ }^{1} \mathrm{H}-\mathrm{NMR}$-Analyse $\left(\mathrm{CDCl}_{3}\right.$ und $\mathrm{d}_{6}$-DMSO) als auch EIMassenanalyse nur 80 bestätigt wurde. Da sich Iodverbindungen bei höherer Temperatur zersetzen können, wurde die Umsetzung unter Zugabe des Phase-Transfer-Katalysators 
TBACl oder TBAI durchgeführt. Unter milden Bedingungen konnte 91 in 96\% Ausbeute isoliert werden, wobei sich noch Spuren $\mathbf{8 0}$ in der Probe befanden.
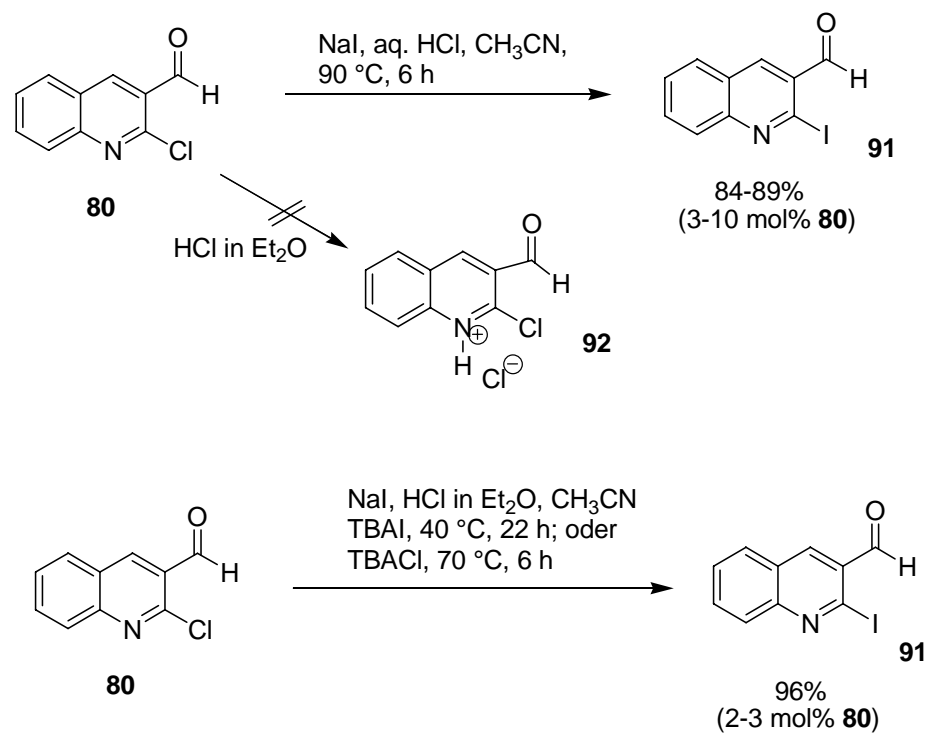

Schema 21. Synthese von 91 durch Iodierung

Für die nachfolgende Heck-Kupplung mit Ethylacrylat lieferte eine Kombination von $\mathrm{Pd}(\mathrm{OAc})_{2}$ und $\mathrm{P}(\text { o-tolyl })_{3}$ das gewünschte Produkt 81 in $65 \%$ Ausbeute. Da Liganden, Lösungsmittel, Temperatur und Katalysator eine Übergangsmetall-katalysierte Reaktion beeinflussen könnten, wurde eine Optimierung der Heck-Reaktion unter Einbeziehung dieser Parameter durchgeführt. (siehe Tabelle 3). Es konnte kaum Produkt isoliert werden, wenn $\mathrm{P}(\text { o-tolyl })_{3}$ durch den Phosphan- Liganden $\mathrm{PPh}_{3}$ ersetzt wurde. Es führte auch der kommerzielle Katalysator $\mathrm{Pd}\left(\mathrm{PPh}_{3}\right)_{4}$ zu keinem signifikanten Umsatz (Eintrag 2 und 3). Die niedrige Ausbeute ist wahrscheinlich auf die Koordinationsfähigkeit des Chinolin-Stickstoffs zurückzuführen, welche in Konkurrenz zur Bindung der Phosphan-Liganden tritt. Der sperrigere Ligand $\mathrm{P}(o \text {-tolyl })_{3}$ konnte diese potenzielle Koordination verhindern und die erwünschte Heck-Kupplung erlauben. Bei der Umsetzung mit 1,4-Dioxan als Lösungsmittel statt DMF unter sonst gleichen Bedingungen konnte das Produkt nur in Spurenmengen detektiert werden (Eintrag 4). Da bei einer Temperatur unterhalb $110{ }^{\circ} \mathrm{C}$ keine Umsetzung in DMF beobachtet werden konnte, wurden keine anderen Lösungsmittel untersucht. Andere Katalysatoren z. B. der Herrmann-Beller-Katalysator ${ }^{62}$ und $\mathrm{Pd}(\mathrm{dppf}) \mathrm{Cl}_{2} \cdot \mathrm{CH}_{2} \mathrm{Cl}_{2}{ }^{63}$ stellten mit jeweils 20\% und 30\% Ausbeute keine Verbesserung dar (Eintrag 5 und 6). Es ist zu beachten, dass die Wahl des Laufmittels für die chromatographische Aufreinigung von 81 von großer Bedeutung war. Mit einem Gemisch von Pentan/EtOAc (4:1) war sehr viel Eluens 
erforderlich. Es trat eine und geringe Zersetzung auf. Hingegen ließ sich mit einem Gemisch von $\mathrm{CH}_{2} \mathrm{Cl}_{2} /$ EtOAc (50:1) das Produkt effektiv und bequem isolieren.

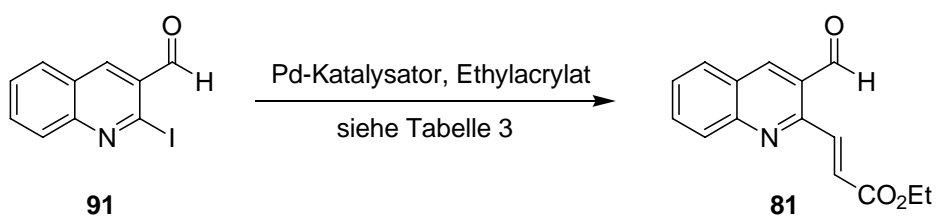

Schema 22. Synthese von 81 durch Heck-Kupplung

\begin{tabular}{|c|c|c|}
\hline Eintrag & Reaktionsbedingungen & Ausbeute \\
\hline 1 & $\begin{array}{l}1.0 \text { Äq. 91, } 2.1 \text { Äq. Ethylacrylat, } 1.5 \text { Äq. NaOAc, } 5.3 \\
\text { mol\% TBAI, } 2.1 \mathrm{~mol} \% \mathrm{Pd}(\mathrm{OAc})_{2}, 4.3 \mathrm{~mol} \% \mathrm{P}(\text { o-tolyl })_{3} \text {, } \\
\text { DMF, } 120{ }^{\circ} \mathrm{C}, 12 \mathrm{~h}\end{array}$ & $65 \%$ \\
\hline 2 & $\begin{array}{l}1.0 \text { Äq. 91, } 2.0 \text { Äq. Ethylacrylat, } 4.0 \text { Äq. } \mathrm{NEt}_{3}, 5.0 \mathrm{~mol} \% \\
\operatorname{Pd}(\mathrm{OAc})_{2}, 12 \mathrm{~mol}^{2} \mathrm{PPh}_{3}, \mathrm{DMF}, 110^{\circ} \mathrm{C}, 19.5 \mathrm{~h}\end{array}$ & Spuren \\
\hline 3 & $\begin{array}{l}1.0 \text { Äq. 91, } 2.0 \text { Äq. Ethylacrylat, } 1.4 \text { Äq. NaOAc, } 2.3 \\
\operatorname{mol} \% \operatorname{Pd}\left(\mathrm{PPh}_{3}\right)_{4}, \mathrm{DMF}, 110^{\circ} \mathrm{C}, 16.5 \mathrm{~h}\end{array}$ & Spuren \\
\hline 4 & $\begin{array}{l}1.0 \text { Äq. 91, } 2.0 \text { Äq. Ethylacrylat, } 1.4 \text { Äq. NaOAc, } 5.0 \\
\text { mol\% TBAI, } 4.5 \mathrm{~mol} \% \mathrm{Pd}(\mathrm{OAc})_{2}, 9.2 \mathrm{~mol} \% \mathrm{P}(o \text {-tolyl })_{3} \text {, } \\
\text { 1,4-Dioxan, } 110^{\circ} \mathrm{C}, 17 \mathrm{~h} \text {, dann } 120^{\circ} \mathrm{C}, 22 \mathrm{~h}\end{array}$ & Spuren \\
\hline 5 & $\begin{array}{l}1.0 \text { Äq. 91, } 1.4 \text { Äq. Ethylacrylat, } 1.1 \text { Äq. NaOAc, } 0.70 \\
\text { mol\% TBABr, } 0.10 \text { mol\% Herrmann-Beller-Katalysator, } \\
\text { DMF, } 125^{\circ} \mathrm{C}, 4 \mathrm{~h}\end{array}$ & $20 \%$ \\
\hline 6 & $\begin{array}{l}1.0 \text { Äq. 91, } 1.5 \text { Äq. Ethylacrylat, } 2.0 \text { Äq. NaOAc, } 6.0 \text { Äq. } \\
\mathrm{H}_{2} \mathrm{O}, 2.0 \mathrm{~mol} \% \mathrm{Pd}(\mathrm{dppf}) \mathrm{Cl}_{2} \cdot \mathrm{CH}_{2} \mathrm{Cl}_{2}, \mathrm{DMF}, 110^{\circ} \mathrm{C}, 5 \mathrm{~h}\end{array}$ & $37 \%$ \\
\hline
\end{tabular}

Tabelle 3. Untersuchung der Synthese von 81 durch Heck-Kupplung 


\subsection{Aufbau des C-Rings}

Der Aufbau des C-Rings (siehe Abbildung 1) sollte mit Benzylamin durch reduktive Aminierung und intramolekulare 1,4-Michael-Addtion erfolgen. Für eine optimale Umsetzung wurde verschiedene Reduktionsmittel untersucht.

\subsubsection{Untersuchung mit verschiedenen Reduktionsmitteln}<smiles>CCOC(=O)/C=C/c1nc2ccccc2cc1C=O</smiles>

81

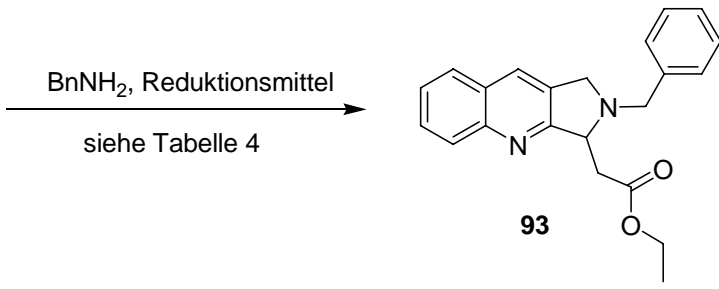

Schema 23. Synthese von 93

Zuerst wurde eine Umsetzung mit $\mathrm{NaBH}_{4}$ durchgeführt. Nach der Iminbildung in Methanol erfolgte die Zugabe von $\mathrm{NaBH}_{4}$ in mehreren Portionen. Trotz der in der Literatur angegeben hohen Ausbeute ${ }^{58}$ wurde festgestellt, dass unabhängig von der Zugabemenge und -geschwindigkeit des Reduktionsmittels eine Vielzahl von Nebenprodukten gebildet wird. Das Produkt 93 konnte auf diese Weise nur in einer Ausbeute von 31\% isoliert werden (siehe Tabelle 4, Eintrag 1). Mit $\mathrm{NaBH}_{3} \mathrm{CN}^{64}$ wurde das Produkt neben der Bildung mehrerer Nebenprodukte nur in Spuren dünnschichtchromatographisch beobachtet. Basierend auf Arbeiten von A. F. Abdel-Magid ${ }^{65}$ konnte durch Verwendung von $\mathrm{NaBH}(\mathrm{OAc})_{3}$ in 1,2Dichlorethan (DCE) mit Benzylammoniumchlorid und Triethylamin die Ausbeute dramatisch verbessert werden (Eintrag 3). Da sich die konkurrierende Aldehydreduktion zum Alkohol nicht ausschließen ließ, die unter sauren Bedingungen beschleunigt werden sollte, wurde die Umsetzung anschließend ohne Säure/Base-Zugabe unter langsamer Zugabe des Reduktionsmittels in mehreren Portionen durchgeführt. So konnte die Ausbeute bis auf $76 \%$ erhöht werden (Eintrag 4). 


\begin{tabular}{|c|c|c|}
\hline Eintrag & Reaktionsbedingungen & Ausbeute \\
\hline 1 & $\begin{array}{l}1.0 \text { Äq. 81, } 1.1 \text { Äq. } \mathrm{BnNH}_{2}, \mathrm{MS}(3 \AA), \mathrm{MeOH}, \mathrm{RT}, 1 \mathrm{~h} \text {; } \\
\text { dann } 2.0 \text { Äq. } \mathrm{NaBH}_{4}, 0^{\circ} \mathrm{C} \text { to RT, } 4.5 \mathrm{~h}\end{array}$ & $31 \%$ \\
\hline 2 & $\begin{array}{l}1.0 \text { Äq. 81, } 2.0 \text { Äq. } \mathrm{BnNH}_{2} \cdot \mathrm{HCl}, 1.0 \text { Äq. } \mathrm{BnNH}_{2}, \mathrm{MS} \\
(3 \AA), \mathrm{MeOH}, 0{ }^{\circ} \mathrm{C}, 50 \mathrm{~min} \text {, dann } 0.70 \text { Äq. } \mathrm{NaBH}_{3} \mathrm{CN} \text {, } \\
0{ }^{\circ} \mathrm{C}, 2 \mathrm{~h} \text {; RT, } 19 \mathrm{~h}\end{array}$ & Spurenmengen \\
\hline 3 & $\begin{array}{l}1.0 \text { Äq. 81, } 1.1 \text { Äq. BnNH }{ }_{2} \cdot \mathrm{HCl}, 1.4 \text { Äq. } \mathrm{NEt}_{3}, 3.0 \text { Äq. } \\
\mathrm{NaBH}(\mathrm{OAc})_{3}, \mathrm{DCE}, 0{ }^{\circ} \mathrm{C} \text { auf RT, } 46 \mathrm{~h}\end{array}$ & $60 \%$ \\
\hline 4 & $\begin{array}{l}1.0 \text { Äq. 81, } 1.5 \text { Äq. } \mathrm{BnNH}_{2} \text {, DCE, } 1.6 \text { Äq. } \mathrm{NaBH}(\mathrm{OAc})_{3} \\
\text { (Zugabe über } 1 \text { h), RT, } 4 \mathrm{~h}\end{array}$ & $76 \%$ \\
\hline
\end{tabular}

Tabelle 4. Synthese von 93 mit verschiedenen Reduktionsmitteln

\subsubsection{Synthese über Imin-Zwischenstufe}

Zur weiteren Optimierung wurde die Umsetzung in zwei separaten Schritten, d. h. einer insitu Iminbildung und nachgefolgten Iminreduktion durchgeführt. Aufgrund der normalerweise schnellen Iminbildung in Methanol wurde die Reaktion nach zwei Stunden bei Raumtemperatur beendet. Nach Entfernung des Lösungsmittels im Vakuum wurden zur Reduktion $\mathrm{H}_{2}$ auf $\mathrm{Pd} / \mathrm{C}$ in Methanol und $\mathrm{NaBH}(\mathrm{OAc})_{3}$ in 1,2-Dichlorethan, Dichlormethan oder Methanol für die Reduktion verwendet. In allen Fällen war eine Vielzahl von Nebenprodukten zu beobachten.

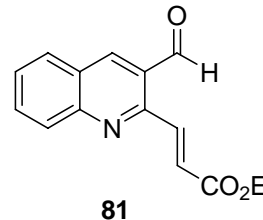

81
$\mathrm{BnNH}_{2}$ in $\mathrm{MeOH}, \mathrm{RT}, 2 \mathrm{~h}$, dann

a) $\mathrm{H}_{2}, \mathrm{Pd} / \mathrm{C}$ in $\mathrm{MeOH}$

b) $\mathrm{NaBH}(\mathrm{OAc})_{3}$ in $\mathrm{DCE}$

c) $\mathrm{NaBH}(\mathrm{OAc})_{3}$ in Dichlormethan

d) $\mathrm{NaBH}(\mathrm{OAc})_{3}$ in Methanol

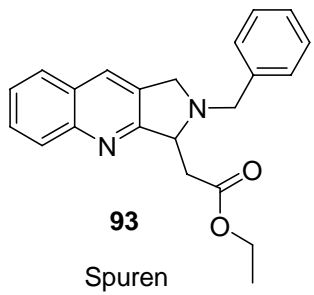

Spuren

Schema 24. Synthese über Imin-Zwischenstufe 


\subsubsection{Bedeutung des Reduktionsmittels}

Die geringe Ausbeute mit $\mathrm{NaBH}_{4}$ ist einerseits auf die Unverträglichkeit der Doppelbindung, ${ }^{66}$ anderseits auf die langsame und reversible Iminbildung zurückzuführen, was die schnelle Aldehydreduktion begünstigen kann. Da keine Imine isoliert werden konnten, kann darauf geschlossen werden, dass auch in-situ nur eine langsame Iminbildung erfolgt. Da die reduktive Aminierung mit $\mathrm{NaBH}_{3} \mathrm{CN}$ saure Bedingungen voraussetzt, kann eine unerwünschte Chinolin-Aktivierung bzw. Reduktion nicht vermieden werden. Diese Unverträglichkeit lässt sich auch bei der Synthese von 105 (siehe Kap. 2.4.1.2, Schema 33) beobachten. Bei Verwendung von $\mathrm{NaBH}(\mathrm{OAc})_{3}$ wird nur eine geringe Reduktionsrate der Doppelbindung beobachtet, daher liefert dies Verfahren die beste Ausbeute.

\subsubsection{Weitere Optimierung durch Zugabetechnik}

Bei einer reduktiven Aminierung ist ein Überschuss an primärem Amin notwendig, um eine zweifache Aminierung zu unterdrücken. Aus diesem Grund wurde das Heck-Produkt 81 als Lösung in 1,2-Dichlorethan äußerst langsam hinzugegeben. Damit konnte die Ausbeute auf $80 \%$ erhöht werden. Außerdem wurde hierdurch die Reaktionsdurchführung erheblich vereinfacht. 


\subsection{Synthese des $N$-Bn-geschützten Aldehyds 94}

\subsubsection{Direkte Reduktion mit DIBAL-H}

Die DIBAL-H-Reduktion von 93 lieferte den gewünschte Aldehyd 94 nach chromatographischer Reinigung höchstens in 59\% Ausbeute, wobei die Anwendung vom überschüssigen DIBAL-H notwendig war, um das Edukt vollständig umzusetzen. Da sich das Produkt als instabil auf Kiesegel bewies, wurde Natriumhydrogensulfit zur Produktextraktion verwendet. Die geringere Ausbeute von 40-53\% stellte aber keine Verbesserung dar.
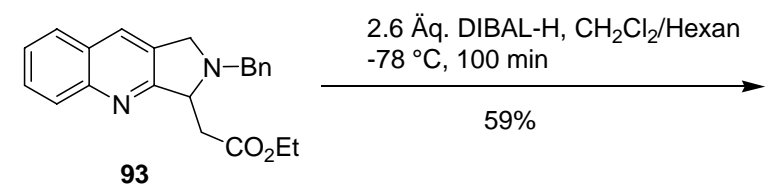

93

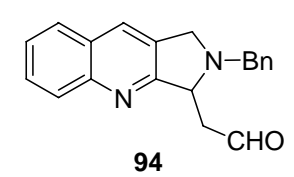

Schema 25. Synthese von 94

\subsubsection{Indirekte Reduktion mit $\mathrm{LiAlH}_{4}$}

Im Hinblick auf einen geringen Anteil des Alkohols 95 bei überschüssigen Mengen an DIBAL-H wurde die Reduktion von 93 mit $\mathrm{LiAlH}_{4}$ zum Alkohol 95 durchgeführt. 95 konnte in 59\% Ausbeute isoliert werden. Dabei trat allerdings Zersetzung auf, welche darauf hinwies, dass das Chinolingerüst nicht geeignet zur $\mathrm{LiAlH}_{4}$-Reduktion war. ${ }^{67}$ Aufgrund der geringen Ausbeute wurde keine weitere Oxidation der Hydroxygruppe zum Aldehyd durchgeführt.

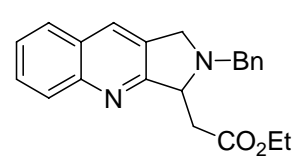

93

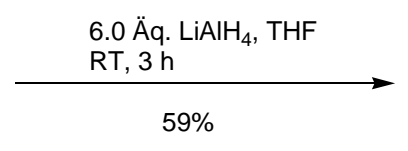

$59 \%$

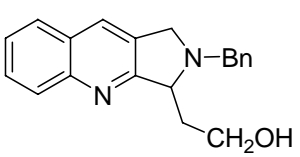

95

Schema 26. Synthese von 95 


\subsection{Synthese des Cbz-geschützten Aldehyds 96}

\subsubsection{Synthese des Cbz-geschützten Esters 97}

Um den $\mathrm{N}$-Cbz-geschützten Aldehyd 96 darzustellen, sollte zuerst der entsprechende Ester 97 synthetisiert werden, der nachher zu 96 reduziert wird. Zur Synthese von 97 wurden zwei unterschiedene Wege A und B untersucht (siehe Schema 27).

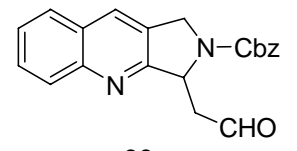

96
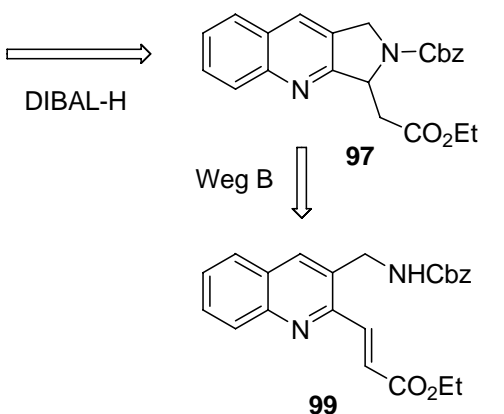
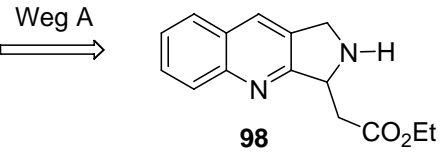

Schema 27. Retrosynthese von 96 über den Cbz-geschützte Ester 97

\subsubsection{Untersuchung zur Synthese des sekundären Amins 98 (Weg A)}

\subsection{Untersuchung der $N$-Debenzylierung von 93}

Zur Synthese des sekundären Amins 98 wurde vor allem die $N$-Debenzylierung von 93 untersucht. Trotz umfangreicher Versuche ließ sich die Benzylgruppe nicht hydrogenolytisch entfernen. Mittels Wasserstoff auf $\mathrm{Pd} / \mathrm{C}$ wurde versucht, die Reaktion anhand des $\mathrm{H}_{2}$-Drucks, des Lösungsmittels und durch Säuren als Additive zu optimieren (siehe Tabelle 5). Dabei wurde gefunden, dass sich das Edukt über längere Zeit zersetzt, während es in kürzerer Zeit (typischerweise zwei Stunden) inert ist. Die Umsetzung unter Zusatz eines Phase-TransferKatalysators führte nicht zu einer Verbesserung.

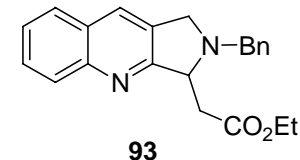

93

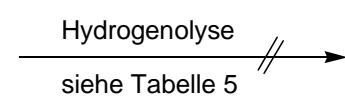

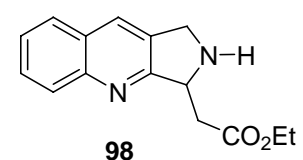

98

Schema 28. Untersuchung der N-Debenzylierung von 93

Ähnliche Probleme bei Debenzylierungen sind ebenfalls literaturbekannt bekannt. ${ }^{68}$ Das ist einerseits auf zwei basische Stickstoffgruppen im Edukt zurückzuführen, welche den $\mathrm{Pd} / \mathrm{C}$ - 
Katalysator deaktivieren können. Andererseits kann die dreifache ,Benzyl-Position' des Stickstoffs innerhalb des C-Rings der gewünschten Debenzylierung entgegenwirken. Außerdem kann die Labilität von 98 auch dafür verantwortlich sein. Es wurde tatsächlich später gefunden, dass sich 98 bei Raumtemperatur schnell zersetzt (siehe Kap. 2.4.1.3).

\begin{tabular}{|c|c|c|}
\hline Eintrag & Reaktionsbedingungen & Ergebnis \\
\hline 1 & $\begin{array}{l}\text { 93, 28\% (bezogen auf Masse) Pd/C (10 Gew.\%), } \\
\mathrm{H}_{2} \text {-Ballon, EtOH, RT, } 22.5 \mathrm{~h}\end{array}$ & Zersetzung \\
\hline 2 & $\begin{array}{l}\text { 93, 17\% (bezogen auf Masse) Pd/C (10 Gew.\%), } \mathrm{H}_{2} \\
(30 \mathrm{psi}), \mathrm{EtOH}, \mathrm{RT}, 120 \mathrm{~min}\end{array}$ & Edukt \\
\hline 3 & $\begin{array}{l}\text { 93, 14\% (bezogen auf Masse) Pd/C (10 Gew.\%), } \mathrm{H}_{2} \\
\text { (50 psi), EtOAc, RT, } 140 \mathrm{~min}\end{array}$ & Edukt \\
\hline 4 & $\begin{array}{l}\text { 93. } \mathrm{HCl} \text { (Salz), 69\% (bezogen auf Masse) } \mathrm{Pd} / \mathrm{C} \\
\left(10 \mathrm{Gew} \% \text { ), } \mathrm{H}_{2}-\text { Ballon, EtOH, RT, } 150 \mathrm{~min}\right.\end{array}$ & Zersetzung \\
\hline 5 & $\begin{array}{l}1.0 \text { Äq. 93, } 56 \% \text { (bezogen auf Masse) } \mathrm{Pd} / \mathrm{C} \\
(10 \text { Gew.\%), } 3.0 \text { Äq. HOAc, } 5.0 \text { Äq. 1,4-Cyclo- } \\
\text { hexadien, EtOH, RT, } 16 \text { min }\end{array}$ & Edukt + Zersetzung \\
\hline
\end{tabular}

Tabelle 5. Untersuchung der N-Debenzylierung von 93

\subsection{Untersuchung der oxidativen Spaltung der $N$-PMB-Gruppe von 100}

Aufgrund des Debenzylierungsproblems wurde dann eine oxidative Spaltung der N-PMBGruppe von 100 untersucht. 100 ließ sich auf die ähnliche Weise wie bei der Synthese von 93 mit para-Methoxybenzylamin herstellen. Unter den beschriebenen optimierten Bedingungen konnte 100 in 92\% Ausbeute isoliert werden (siehe Schema 29). Im Vergleich zur obigen Synthese von 93 (80\% Ausbeute mit Benzylamin, siehe Kap. 2.2.4) ist die bessere Ausbeute vermutlich auf die höhere Nucleophilie des Stickstoffes in para-Methoxybenzylamin zurückzuführen. Allerdings konnte die $N$-PMB-Gruppe durch anschließende Umsetzungen mit CAN oder DDQ nicht oxidativ entfernt werden. Im wesentlichen wurde das Edukt zurückgewonnen. Die Reaktion mit $\mathrm{H}_{2}$ und $\mathrm{Pd}(\mathrm{OH})_{2} / \mathrm{C}$ führte erwartungsgemäß ebenfalls nicht zu einer Spaltung. 
<smiles>CCOC(=O)/C=C/c1nc2ccccc2cc1C=O</smiles>

81

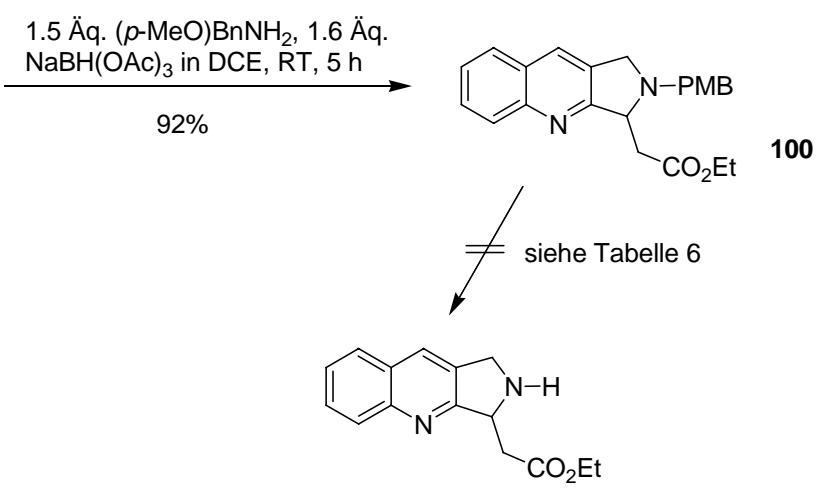

98

Schema 29. Die Synthese von 100 und Untersuchung der oxidativen Spaltung der N-PMB-Gruppe

\begin{tabular}{clc}
\hline Eintrag & \multicolumn{1}{c}{ Reaktionsbedingungen } & Ergebnis \\
\hline 1 & 1.0 Äq. 100, 1.2 Äq. DDQ, $\mathrm{CH}_{2} \mathrm{Cl}_{2} / \mathrm{H}_{2} \mathrm{O}(5: 1), \mathrm{RT}, 2 \mathrm{~h}$ & Edukt \\
2 & $\begin{array}{l}1.0 \text { Äq. 100, 2.1 Äq. CAN, } \mathrm{CH}_{3} \mathrm{CN} / \mathrm{H}_{2} \mathrm{O}(5: 1), 0^{\circ} \mathrm{C}, 1 \mathrm{~h} ; \\
\text { dann RT, 2 h }\end{array}$ & Edukt \\
3 & $\begin{array}{l}\mathbf{1 0 0}, 35 \% \text { (bezogen auf Masse) } \mathrm{Pd}(\mathrm{OH})_{2} / \mathrm{C}(20 \mathrm{Gew} \%), \\
\text { EtOH, RT, } 5 \mathrm{~h}\end{array}$ & Edukt \\
\hline
\end{tabular}

Tabelle 6. Untersuchung der oxidativen Spaltung der $N$-PMB-Gruppe von 100

\subsection{Direkte Einführung der Cbz-Gruppe in 81}

Zur Untersuchung der direkten Synthese von 98 aus 81 wurde Ammoniumsalze $\left(\mathrm{NH}_{4} \mathrm{Cl}\right.$ und $\mathrm{NH}_{4} \mathrm{OAc}$ ) und aq. Ammoniak-Lösung verwendet (siehe Schema 30). Es ließ sich keine Bildung von 98 beobachten. Mit $\mathrm{NaBH}(\mathrm{OAc})_{3}$ wurde ausschließlich die Reduktion der Aldehydgruppe zum Alkohol 101 bestätigt. Mit $\mathrm{NaBH}_{3} \mathrm{CN}$ konnten die Nebenprodukte von 102 und 101 in jeweils 14\% bzw. 17\% Ausbeute isoliert werden. Die Bildung von 102 konnte durch zweifache reduktive Aminierung und intramolekulare Cyclisierung erklärt werden. Das gewünschte sekundäre Amin 98 wird in situ gebildet. Aufgrund der stärkeren Nucleophile von $98 \mathrm{im}$ Vergleich zu den Ammoniumsalzen konnte 98 aber nicht unter den Bedingungen isoliert werden. Deshalb wurde auf weitere Versuche in dieser Richtung verzichtet. 
<smiles>CCOC(=O)/C=C/c1nc2ccccc2cc1C=O</smiles>

81

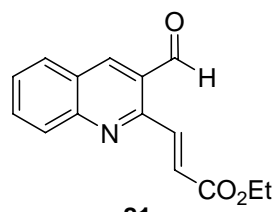

81

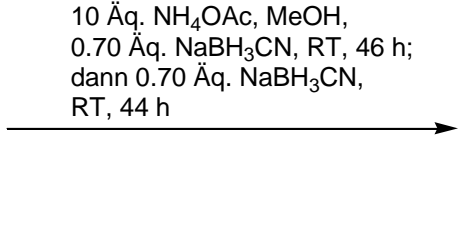
dann 0.70 Äq. $\mathrm{NaBH}_{3} \mathrm{CN}$<smiles>CCOC(=O)C=Cc1nc2ccccc2cc1CO</smiles>

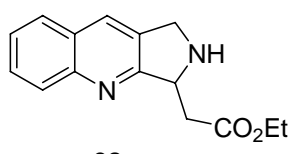

98

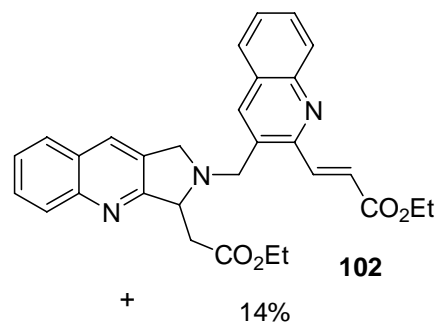<smiles>CCOC(=O)/C=C/c1nc2ccccc2cc1CO</smiles>

$17 \% \quad 101$

Schema 30. Untersuchung ausgehend von Heck-Produkt 81 zur Synthese von 98

\subsubsection{Versuch der Synthese von 99 (Weg B)}

Da Weg A über die Synthese des sekundären Amins 98 soweit erfolglos blieb, wurde der Weg B über die Synthese des Esters 99 untersucht, welcher aus 81 oder 103 hergestellt werden konnte.<smiles>CC(=O)N1Cc2cc3ccccc3nc2C1CC=O</smiles>

96

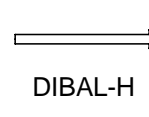

DIBAL-H

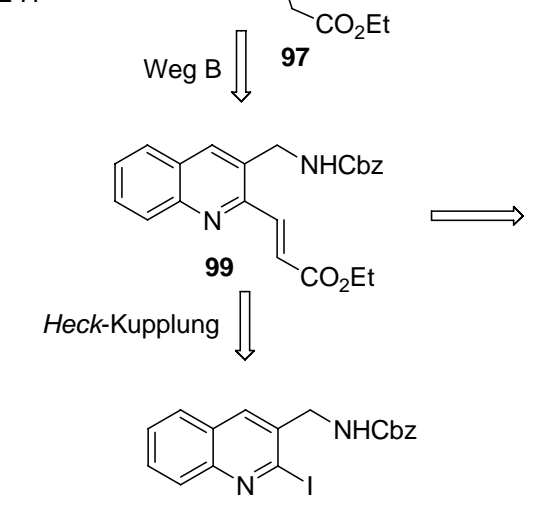

103

Schema 31. Retrosynthese von 96 über 99 
Ausgehend von dem Heck-Produkt 81 wurde zuerst eine reduktive Aminierung mit dem Benzylcarbamat 113 und $\mathrm{NaBH}(\mathrm{OAc})_{3}$ untersucht. Es konnte keine Reaktion beobachtet werden, was auf die geringe Nucleophilie des Stickstoffs im Benzylcarbamat 113 zurückzuführen ist. Durch die Zugabe von Essigsäure konnte auch keine Verbesserung erreicht werden. Umsetzungen unter Verwendung von TFA und $\mathrm{Et}_{3} \mathrm{SiH}^{69}$ lieferten nicht 99, sondern nur 104 in 31\% Ausbeute.

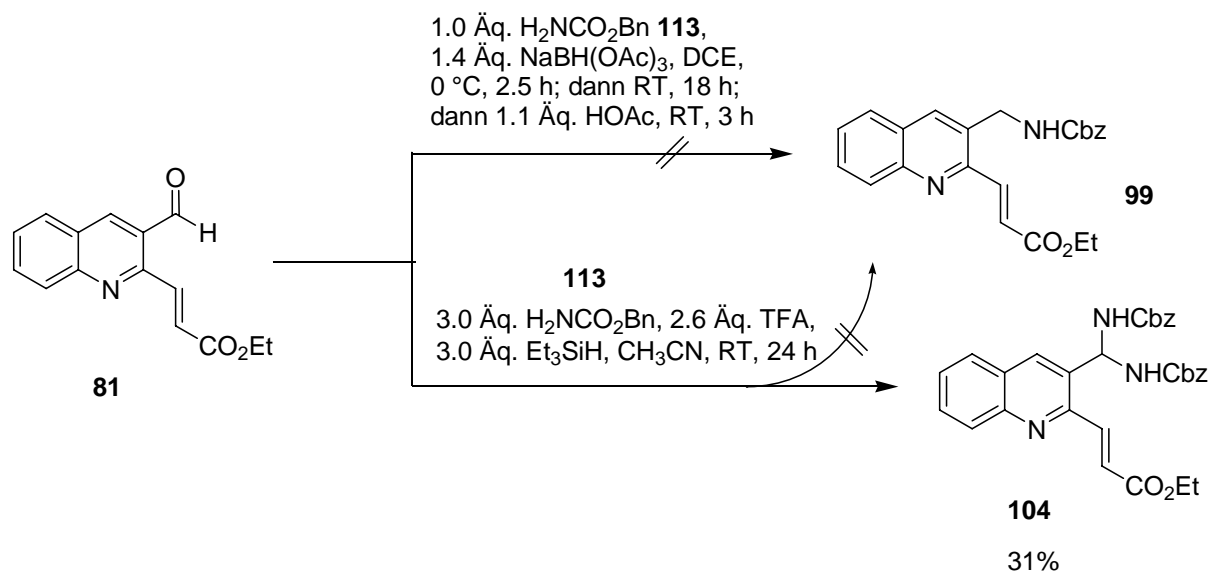

Schema 32. Versuch der Synthese von 99 aus 81

Anschließend wurde die Synthese von 103 untersucht. Die reduktive Aminierung von 91 mit Benzylamin gab, abhängig vom eingesetzten Reduktionsmitteln, das Amin 105 in jeweils 80\% bzw. 64\% Ausbeute (siehe Schema 33). Bei der weiteren Umsetzung des Amins unter hydrogenolytischen Bedingungen fand keine Debenzylierung, sondern Dehalogenierung zu 106 in statt. Die modifizierte reduktive Aminierung von 91 mit $p$-Methoxybenzylamin und $\mathrm{NaBH}_{4}$ führte zwar zum Amin 107 in quantitativer Ausbeute, die anschließende Spaltung der PMB-Gruppe mit CAN, DDQ oder TFA gelang aber nicht. Die weitere Umsetzung des Amins 107 mit Benzylchlorformiat $(\mathrm{Cbz}-\mathrm{Cl})$ lieferte 108 in quantitativer Ausbeute. Die anschließende oxidative PMB-Entschützung von 108 gelang jedoch nicht. 
<smiles>O=Cc1cc2ccccc2nc1I</smiles>

91

$$
\downarrow
$$<smiles>CCCCCCCNCc1cc2ccccc2nc1I</smiles>

107 quant. a) 1.4 Äq. $\mathrm{BnNH}_{2}, 1.6$ Äq. $\mathrm{NaBH}(\mathrm{OAC})_{3}$, DCE, RT, $23 \mathrm{~h}, 80 \%$

oder b) 5.0 Äq. BnNH2, 2.0 Äq

$\mathrm{BnNH}_{2} \cdot \mathrm{HCl}, \mathrm{MS}(3 \AA)$,

$\mathrm{MeOH}, \mathrm{RT}, 4 \mathrm{~h}$; dann

0.7 Äq. $\mathrm{NaBH}_{3} \mathrm{CN}$,

RT, 24 h, 64\%<smiles>Ic1nc2ccccc2cc1CNCc1ccccc1</smiles>

109

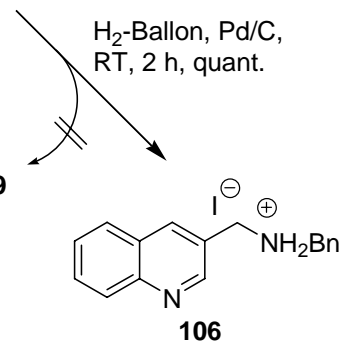

106

1.1 Äq. (p-OMe) $\mathrm{BnNH}_{2}$, $\mathrm{MeOH}, \mathrm{RT}, 4 \mathrm{~h}$; dann 1.6 Äq. $\mathrm{NaBH}_{4}, 0^{\circ} \mathrm{C}, 40 \mathrm{~min}$,

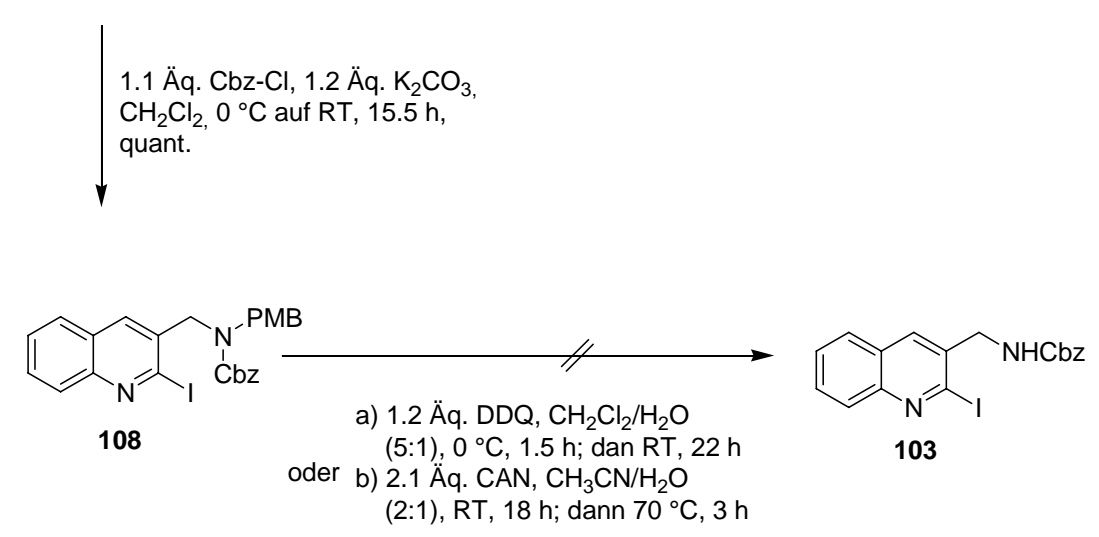

a) 1.2 Äq. DDQ, $\mathrm{CH}_{2} \mathrm{Cl}_{2} / \mathrm{H}_{2} \mathrm{O}$

oder (10:1), RT, $40 \mathrm{~min}$

b) 2.5 Äq. $\mathrm{CAN}, \mathrm{CH}_{3} \mathrm{CN} / \mathrm{H}_{2} \mathrm{O}$

(2:1), RT, $5 \mathrm{~h}$

oder c) 95 Äq. TFA, $60^{\circ} \mathrm{C}, 4.5 \mathrm{~h}$
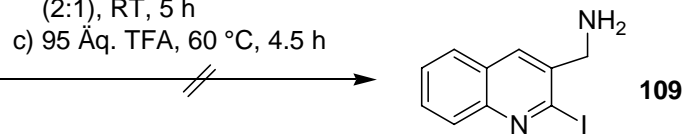

109

(2:1), RT, $18 \mathrm{~h}$; dann $70^{\circ} \mathrm{C}, 3 \mathrm{~h}$

Schema 33. Untersuchung der Synthese von 103 ausgehend von 91

\subsubsection{Synthese des $N$-Cbz-geschützten Esters 97 durch direkte Umschützung}

Eine Umschützung des tertiären Amins 93 mit 2,2,2-Trichlorethylchlorformiat (Troc-Cl) ${ }^{70}$ stellte einen guten Syntheseweg zum N-Troc-geschützten Amin 110 dar. Somit kann 110 in 74\% Ausbeute dargestellt werden. Nach anschließender Troc-Entschützung unter StandardBedingungen mit Zn/HOAc konnte das sekundäre Amin 98 in 45\% Ausbeute isoliert werden. Dabei war zu beachten, dass sich das Amin 98 bereits bei Raumtemperatur langsam zersetzt. Die weitere Umsetzung des Amins mit Cbz-Cl lieferte den $\mathrm{N}$-Cbz-geschützte Ester 97 in quantitativer Ausbeute. Aufgrund der ähnlichen Struktur zwischen $\mathrm{Cbz}-\mathrm{Cl}$ und Troc-Cl wurde eine direkte Umschützung der $N$-Bn-Gruppe in 93 mit $\mathrm{Cbz}-\mathrm{Cl}$ durchgeführt. Der $N$-Cbzgeschützte Ester 97 konnte in 78\% Ausbeute isoliert werden. 


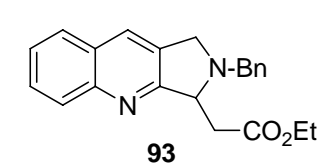

93

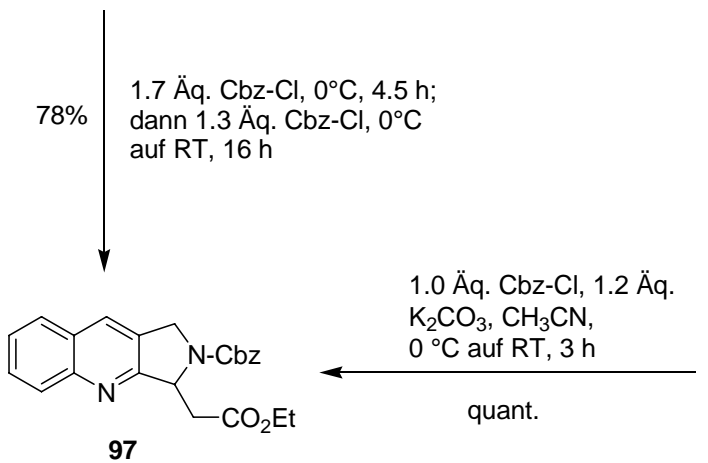

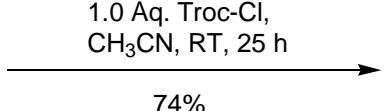

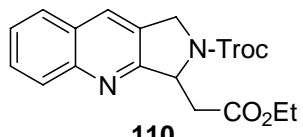

110

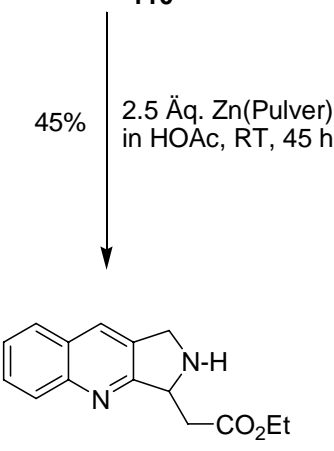

98

Schema 34. Synthese des N-Cbz-geschützten Esters 97 mit Troc-Cl und Cbz-Cl

Weitere Optimierung der Ausbeute ließ sich nicht erreichen, da eine durch die Chinolinstickstoff-Gruppe ausgelöste Nebenreaktion nicht vermieden werden konnte. Testreaktionen von Pyridin und DMAP mit $\mathrm{Cbz}-\mathrm{Cl}$ bestätigten diesen Umstand. Bei dünnschichtchromatographischer Reaktionskontrolle wurde bei Pyridin eine partielle Bildung der sehr polaren Verbindung 111 (vermutlich), und bei DMAP eine vollständige Bildung der sehr polaren Verbindung 112 (vermutlich) beobachtet.

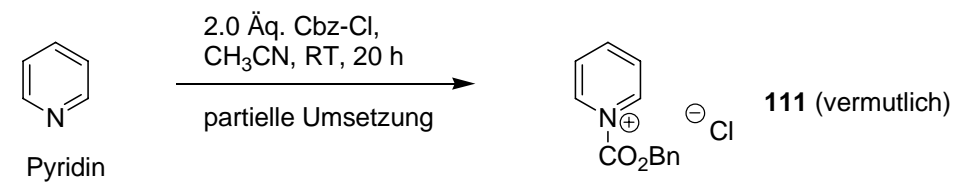

dunkel-violette Lösung

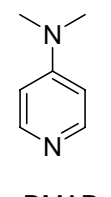

2.0 Äq. Cbz-Cl,

$\underset{\text { vollständige Umsetzung }}{\mathrm{CH}_{3} \mathrm{CN}, \mathrm{RT}, 30 \mathrm{~min}}$

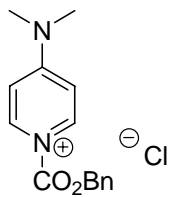

112 (vermutlich)

DMAP

farbloser Niederschlag

Schema 35. Testreaktionen von Pyridin und DMAP mit Cbz-Cl 


\subsubsection{Versuch der Reduktion des $\boldsymbol{N}$-Cbz-geschützten Esters 97}

Die Reduktion von 97 unter Verwendung von DIBAL-H wurde sorgfältig untersucht (siehe Tabelle 7). Es wurde festgestellt, dass keine Reaktion mit stöchiometrischem DIBAL-H in Toluol oder Dichlormethan bei $-78^{\circ} \mathrm{C}$ oder $-60^{\circ} \mathrm{C}$ stattfindet. Ein Überschüss an DIBAL-H führte zu einer Vielzahl von Nebenprodukten und erhöhte Temperatur erlaubte ebenfalls keine Verbesserung.

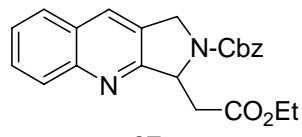

97

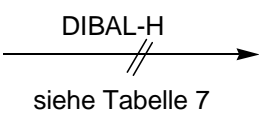

siehe Tabelle 7

Schema 36. Versuche der Reduktion von 97

\begin{tabular}{|c|c|c|}
\hline Eintrag & Reaktionsbedingungen & 96 \\
\hline 1 & $\begin{array}{l}\text { 1.0 Äq. 97, } 1.0 \text { Äq. DIBAL-H, Toluol, }-78^{\circ} \mathrm{C}, 1 \mathrm{~h} \text {; dann } 1.0 \\
\text { Äq. DIBAL-H, }-78{ }^{\circ} \mathrm{C}, 1 \mathrm{~h} \text {; dann } 1.0 \text { Äq. DIBAL-H, }-78^{\circ} \mathrm{C} \text {, } \\
1 \mathrm{~h} \text {; dann RT, } 1.5 \mathrm{~h}\end{array}$ & - \\
\hline 2 & $\begin{array}{l}1.0 \text { Äq. 97, } 1.4 \text { Äq. DIBAL-H, Dichlormethan, }-78^{\circ} \mathrm{C} \text {, } \\
25 \text { min; dann } 1.9 \text { Äq. DIBAL-H, }-78^{\circ} \mathrm{C}, 2 \mathrm{~h}\end{array}$ & - \\
\hline 3 & $\begin{array}{l}1.0 \text { Äq. 97, } 1.0 \text { Äq. DIBAL-H, Dichlormethan, }-78^{\circ} \mathrm{C}, 1 \mathrm{~h} \text {; } \\
\text { dann 1.0 Äq. DIBAL-H, }-78^{\circ} \mathrm{C}, 3 \mathrm{~h} \text {; dann RT, } 17 \mathrm{~h}\end{array}$ & - \\
\hline 4 & $\begin{array}{l}1.0 \text { Äq. 97, } 1.4 \text { Äq. DIBAL-H, Dichlormethan, }-60^{\circ} \mathrm{C}, 2.5 \mathrm{~h} \text {; } \\
\text { dann } 0.60 \text { Äq. DIBAL-H, }-60^{\circ} \mathrm{C}, 1 \mathrm{~h}\end{array}$ & - \\
\hline
\end{tabular}

Tabelle 7. Versuche der Reduktion von $\mathbf{9 7}$ 


\subsubsection{Synthese des $\boldsymbol{N}$-Cbz-geschützten Aldehyds 96 durch direkte Umschützung}

Basierend auf obige Umschützungsversuche mit $N$-Dealkylierungsreagenzien ergab sich dann zur Synthese von 96 ein neuer Weg durch die Umschützung des $N$-Bn-geschützten Aldehyds 94 mit Cbz-Cl. Die Umsetzung lieferte den gewünschten Aldehyd in 80\% Ausbeute.

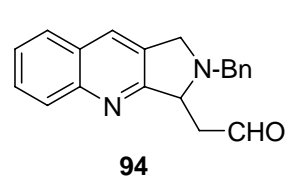

94

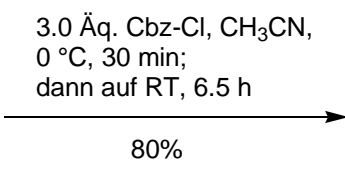

$80 \%$

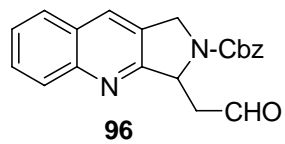

48\% über zwei Stufen ausgehend von 93

Schema 37. Synthese von 96

Aufgrund der Labilität von 94 wurden die nachfolgende Umsetzungen ohne Reinigung von 94 durchgeführt. Ausgehend vom N-Bn-geschützten Ester 93 ließ sich 96 in 63\% Ausbeute über zwei Stufen herstellen. Ausgehend vom N-PMB-geschützte Ester 100 konnte ebenfalls 96 in 64\% Ausbeute auf ähnliche Weise erhalten werden.

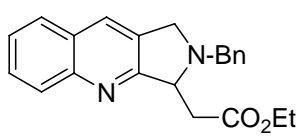

93

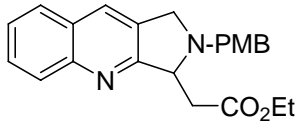

100
1) $1.2 \mathrm{Äq}$ DIBAL-H, $\mathrm{CH}_{2} \mathrm{Cl}_{2},-78{ }^{\circ} \mathrm{C}, 80 \mathrm{~min}$

2) 3.00 Äq. Cbz-Cl, 0.19 Äq. $\mathrm{K}_{2} \mathrm{CO}_{3}, \mathrm{CH}_{3} \mathrm{CN}$, $0{ }^{\circ} \mathrm{C}, 70 \mathrm{~min}$; dann $\mathrm{RT}, 14 \mathrm{~h}$

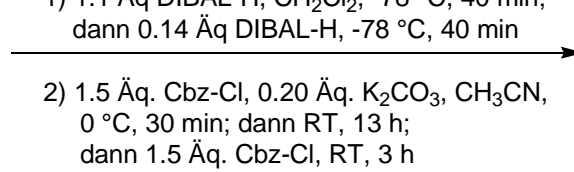

$0^{\circ} \mathrm{C}, 30 \mathrm{~min}$; dann RT, $13 \mathrm{~h}$

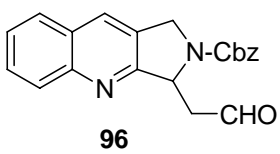

63\% über zwei Stufen

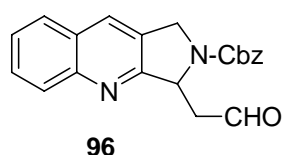

64\% über zwei Stufen

Schema 38. Optimierte Synthese von 96 


\subsubsection{Diskussion ausgewählter spektroskopischer Daten der Verbindung 100}

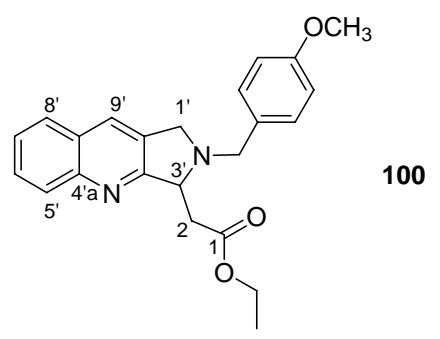

Das ${ }^{1}$ H-NMR-Spektrum (siehe Abbildung 7) der Verbindung 100 zeigt im Tieffeldbereich bei $\delta=6.84-8.04$ die Signale mit dem typischen Kopplungsmuster der Chinolin-Einheit und des para-substituierten Aromaten. Das Singulett bei $\delta=7.79$ lässt sich dem Proton an C-9' zuordnen. Über NOESY-Experimente konnten das Proton an C-8' bei $\delta=7.73$ als Dublett mit einer ortho-Kopplungskonstante von $J=8.0 \mathrm{~Hz}$ sowie die zwei diastereotopen 1 '- $\mathrm{H}$ in dem Multiplettbereich bei $\delta=3.61-3.75$ und 4.11-4.27 identifiziert werden. Das Proton an C-5, resoniert bei $\delta=8.04$ in Form eines Dubletts mit einer ortho-Kopplungskonstante von $J=8.5$ Hz. Über ein ${ }^{1} \mathrm{H}^{-1} \mathrm{H}-$ Korrelationsexperiment ließen sich $6^{\prime}-\mathrm{H}$ und $7^{\prime}-\mathrm{H}$ als zentrietes Multiplett jeweils bei $\delta=7.63$ und 7.46 identifizieren. Zwei Protonenpaare am Benzolring bzw. an der meta-Position (zur Methylengruppe) und ortho-Position sind jeweils chemisch äquivalent. Sie resonieren in Form eines Multipletts jeweils bei $\delta=6.84-6.92$ und 7.29-7.36. Die Zuordnung wurde durch ein selektives ${ }^{1} \mathrm{H}$-NOESY-Experiment der sich an der paraPosition befindlichen Methoxygruppe bestätigt. Der Methoxygruppe wird das Singulett bei $\delta$ $=3.80$ zugeordnet. Das Proton an C-3' resoniert als zentriertes Multiplett bei $\delta=4.52$ und korreliert im ${ }^{1} \mathrm{H}-{ }^{1} \mathrm{H}-\mathrm{COSY}$-Spektrum mit zwei diastereotopen Protone an C-2, die beide als Dublett von Dublett jeweils bei $\delta=3.25\left({ }^{2} J=15.7 \mathrm{~Hz}\right.$ und $\left.{ }^{3} J=4.8 \mathrm{~Hz}\right)$ und $2.97\left({ }^{2} J=15.7\right.$ $\mathrm{Hz}$ und ${ }^{3} J=5.9 \mathrm{~Hz}$ ) vorliegen. Die Protonen der Methylgruppe treten als Triplett bei $\delta=1.20$ in Resonanz. Die restlichen Protonen der Methylengruppe befinden sich in dem Multiplettbereich bei $\delta=3.61-3.75$ und 4.11-4.27.

Die Signale des ${ }^{13} \mathrm{C}$-Spektrums konnten durch ${ }^{13} \mathrm{C},{ }^{1} \mathrm{H}-$ Korrelation zugeordnet werden. So findet man im Hochfeldbereich die Signale für die Methylgruppe und Methoxygruppe bei $\delta=$ 14.13 und 55.19 sowie die Signale für die Methylengruppen bzw. C-2, C-1', $\mathrm{CH}_{2} \mathrm{Ph}$ und $\mathrm{OCH}_{2}$ jeweils bei $\delta=38.84,55.38,57.92$ und 60.32. Das Kohlenstoffatom C-3' resoniert bei $\delta=65.07$. Im Tieffeldbereich lassen sich zwei Paare Kohlenstoffatome am Benzolring an der meta-Position (zur Methylengruppe) sowie ortho-Position bei $\delta=113.7$ bzw. 129.9 identifizieren. Die Kohlenstoffatome C-7' und C-5' resonieren bei $\delta=125.9$ bzw. 129.0. Die 
Signale für C-6' und C-9' treten zusammen bei $\delta=128.6$ auf. Das Signal bei $\delta=127.6$ mit höherer Intensität lässt sich C-8' und C-9'a zuordnen. Charakteristisch für die Estergruppe und C-3'a sind chemische Verschiebungen bei $\delta=172.0$ bzw. 164.3. Die restlichen Kohlenstoffatome resonieren bei $\delta=130.6,131.1,147.8$ und 158.8.

Entsprechend weist das IR-Spektrum eine Bande bei $\widetilde{v}=1730 \mathrm{~cm}^{-1}$ für die $\mathrm{C}=\mathrm{O}$ Streckschwingung auf. Das EI-Massenspektrum weist bei $\mathrm{m} / \mathrm{z}=376.4$ einen Peak für das Molekülion auf. Die Peaks bei $\mathrm{m} / \mathrm{z}=255.3$ und 121.2 lassen sich jeweils den Fragmenten $\left[\mathrm{M}-\mathrm{CH}_{2} \mathrm{Ph}\left(p-\mathrm{OCH}_{3}\right)\right]^{+}$und $\left[\mathrm{CH}_{2} \mathrm{Ph}\left(p-\mathrm{OCH}_{3}\right)\right]^{+}$zuordnen.

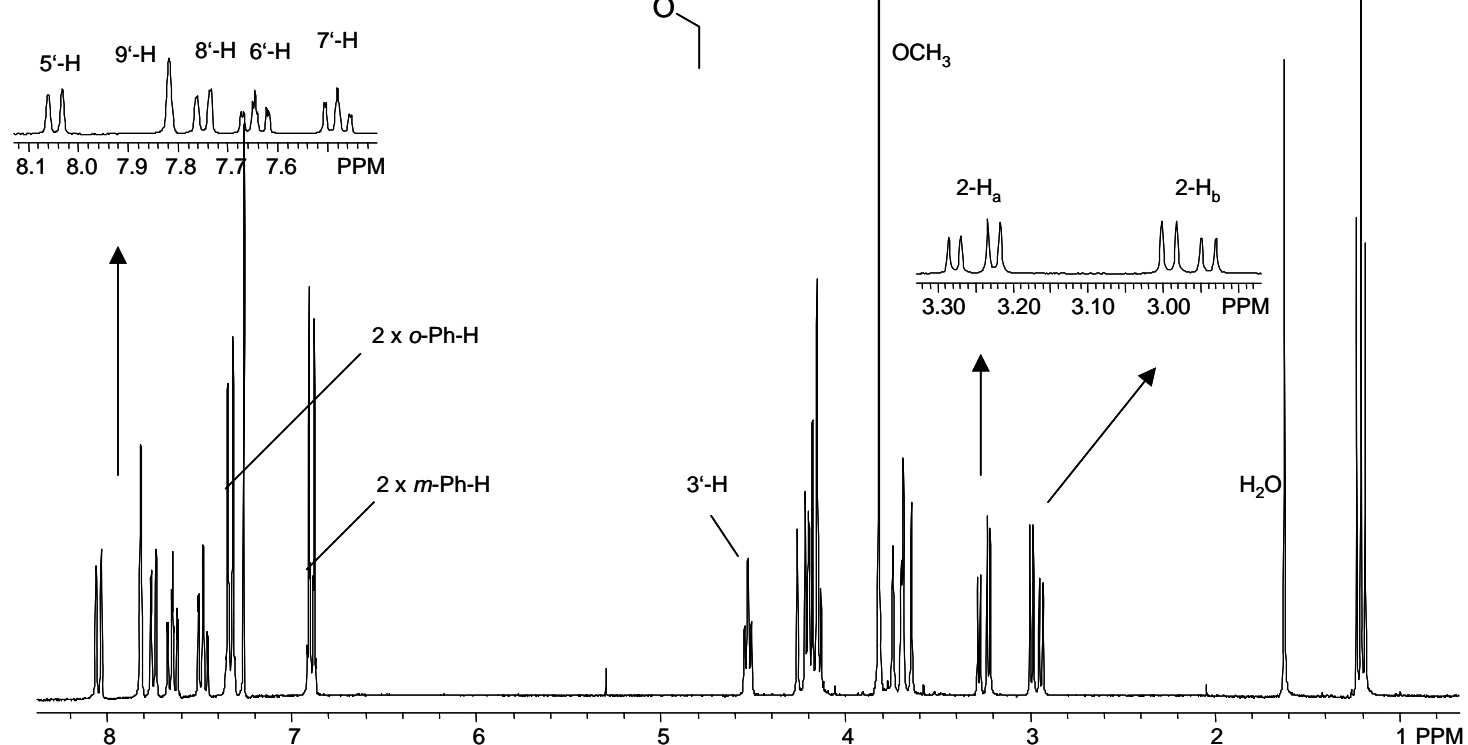

Abbildung 7. ${ }^{1} \mathrm{H}-\mathrm{NMR}\left(\mathrm{CDCl}_{3}, 300 \mathrm{M}\right)$ von $\mathbf{1 0 0}$ 


\subsubsection{Diskussion ausgewählter spektroskopischer Daten der Verbindung 96}

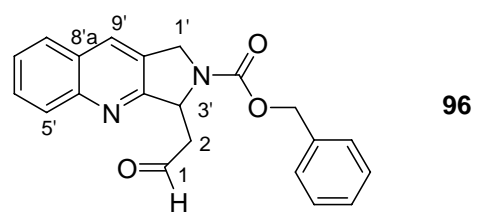

Im ${ }^{1}$ H-NMR-Spektrum des Aldehyds 96 (siehe Abbildung 8) liegen zwei Rotamere A und B im Verhältnis 0.38:0.62 vor, was auf die Carbamatgruppe zurückzuführen ist. Mittels hochaufgelösten ${ }^{1} \mathrm{H}-\mathrm{NMR}$ (600 MHz) werden zwei Rotamere im Tieffeld mit zwei separaten Singuletts bei jeweils $\delta=9.80$ und 9.65 aufgelöst, die charakteristisch für das Aldehydproton sind. Entsprechend sind im Hochfeld vier Resonanzsignale (alle als Dublett von Dublett) jeweils bei $\delta=3.14$ und 3.21 im Verhältnis 0.38:0.62 sowie bei $\delta=3.30$ und 3.54 im gleichen Verhältnis sichtbar. Sie lassen sich den diastereotopen Protonen an C-2 zuordnen. Es kann keine Kopplung zwischen dem Aldehydproton und 2-H beobachtet werden, was durch die beschränkten Torsionswinkel verursachtet werden kann. Außer für 9'-H treten die restlichen Protonen als Multiplett auf. Mit Hilfe einer ${ }^{1} \mathrm{H}-{ }^{1} \mathrm{H}$ und ${ }^{13} \mathrm{C}-{ }^{1} \mathrm{H}-$ Korrelation lassen sie sich trotzdem gut zuordnen.

Im ${ }^{13} \mathrm{C}$-Spektrum werden aufgrund des Vorliegens der beiden Rotamere vom Tieffeld zum Hochfeld immer zwei nebeneinander stehende Signale für ein einzelnes Kohlenstoffatom beobachtet. Die chemische Verschiebung bei $\delta=199.22$, 199.52 ist charakteristisch für die Aldehydgruppe sowie 160.73 und 161.06 für C-3'a. Ausnahmsweise resoniert die Carbamatgruppe als ein Signal bei $\delta=148.00$ ppm mit verstärkter Intensität. Das Aufheizen der Probe auf $100{ }^{\circ} \mathrm{C}$ führt zur Koaleszenz der Rotamer-bedingten doppelten Signale (siehe Abbildung 9), weswegen darauf geschlossen werden kann, dass bei dieser Temperatur die Rotationsbarriere überwunden wird. Das hoch aufgelöste ESI-Massenspektrum weist ein Signal bei $m / z=347.1390$ für $[\mathrm{M}+\mathrm{H}]^{+}($Ber. $m / z=347.1390)$ auf. 


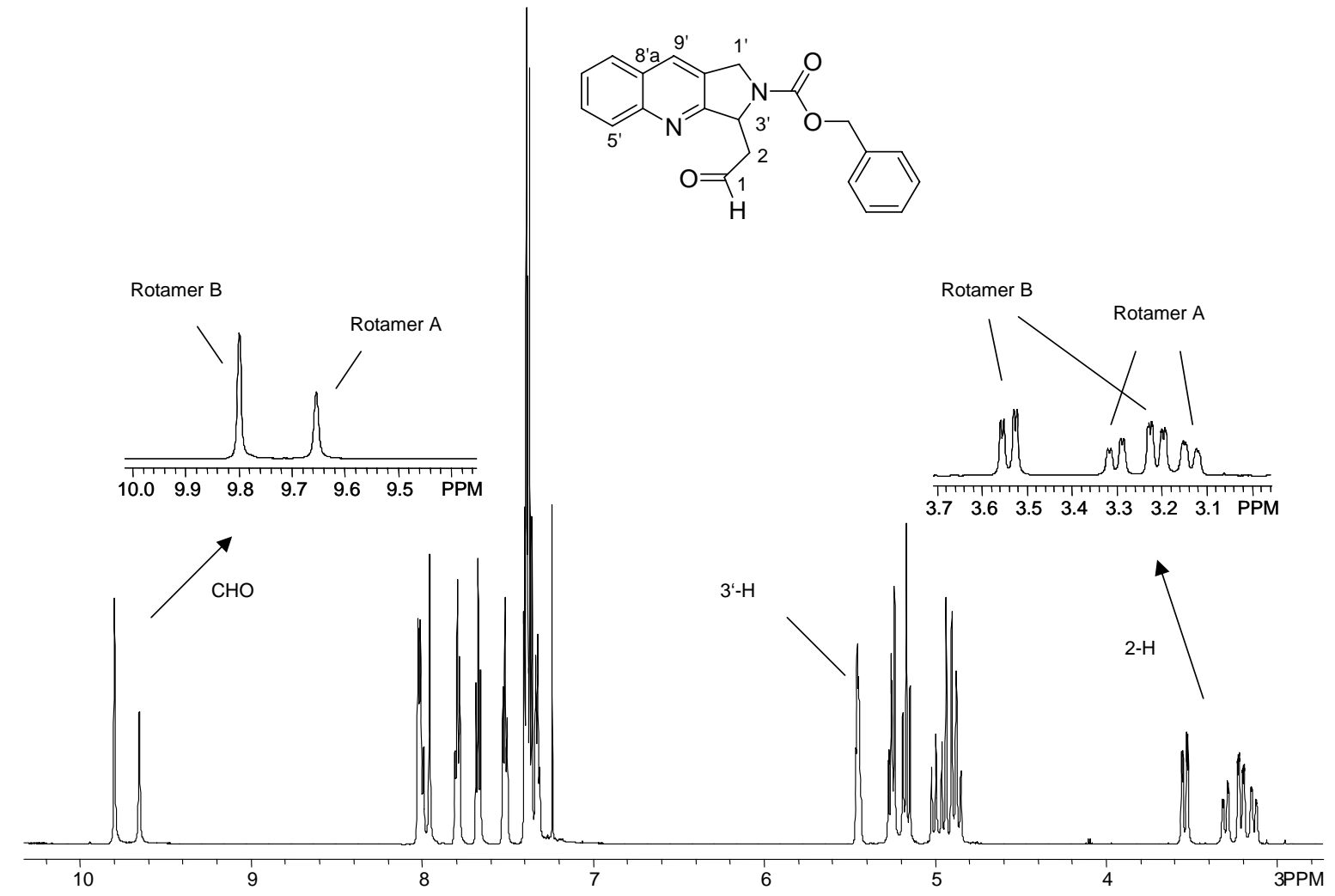

Abbildung 8. ${ }^{1} \mathrm{H}-\mathrm{NMR}\left(\mathrm{CDCl}_{3}, 600 \mathrm{M}\right)$ von 96 bei Raumtemperatur

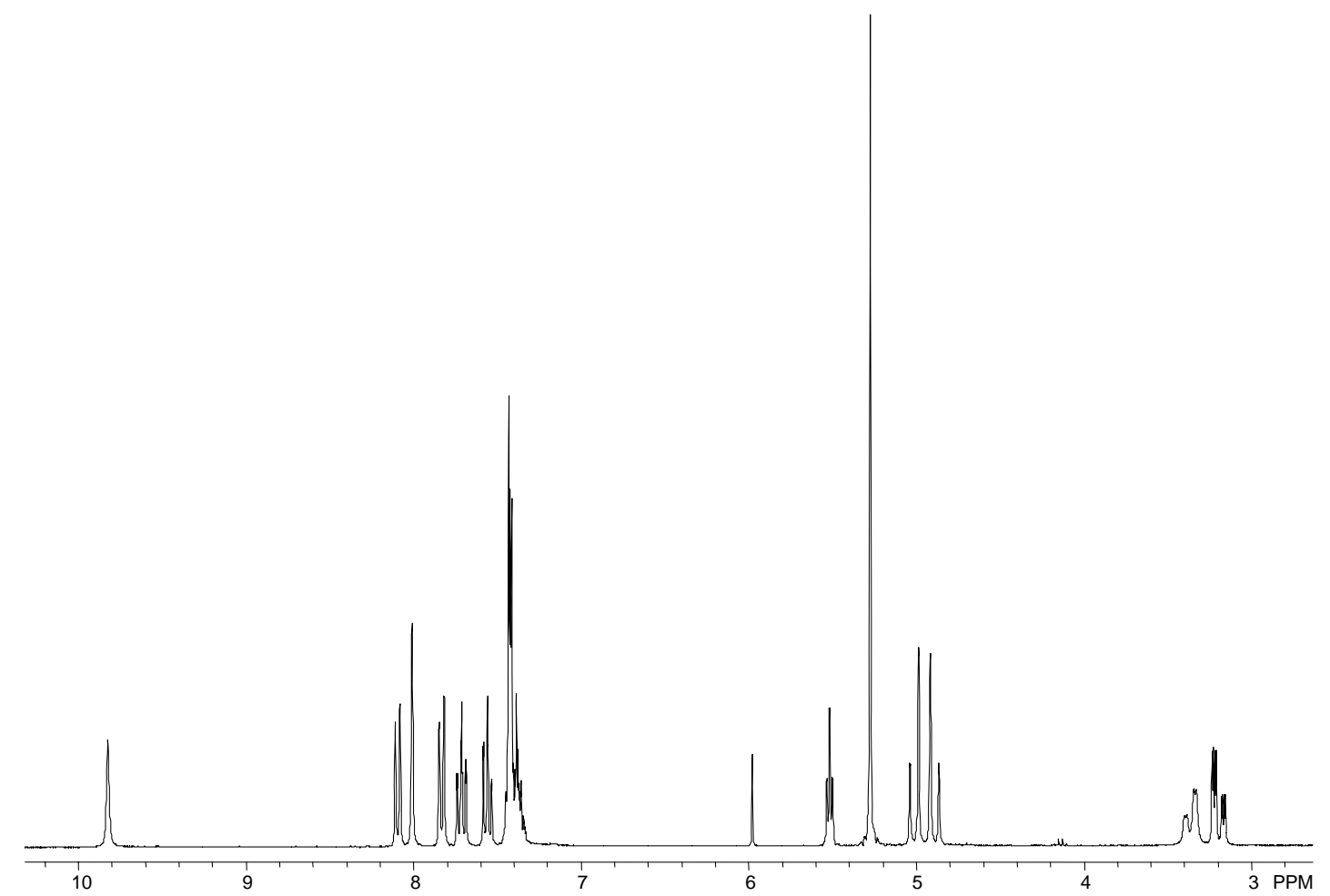

Abbildung 9. ${ }^{1} \mathrm{H}-\mathrm{NMR}\left(\mathrm{C}_{2} \mathrm{D}_{2} \mathrm{Cl}_{4}, 300 \mathrm{M}\right)$ von 96 bei $100{ }^{\circ} \mathrm{C}$ 


\subsection{Synthese der $N$-Troc- und N-ACE-geschützten Aldehyde 114 und 115}

Neben dem N-Cbz-geschützten Aldehyd 96 ließen sich andere Aldehyde auch mit $\mathrm{N}$ Dealkylierungsreagenzien herstellen. Ausgewählt wurden der $N$-Troc-geschützte Aldehyd 114 und N-ACE-geschützte Aldehyd 115. Der N-Bn-geschützte Ester 93 konnte über die DIBAL-H-Reduktion und anschließende Umschützung mit Troc-Cl in $\mathbf{1 1 4}$ mit einer Ausbeute von 46\% überführt werden (siehe Tabelle 8). Ausgehend vom N-PMB-geschützten Ester 100 konnte 114 in 68\% Ausbeute dargestellt werden. Ähnliche Umsetzung von 100 mit 1-Chlorethylchlorformiat (ACE-Cl) ${ }^{71}$ lieferte 115 in 54\% Ausbeute.

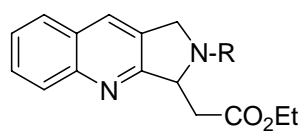
$\begin{aligned} \text { 93: } R & =B n \\ \text { 100: } R & =P M B\end{aligned}$

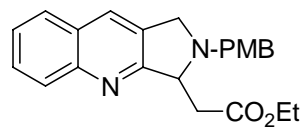

100

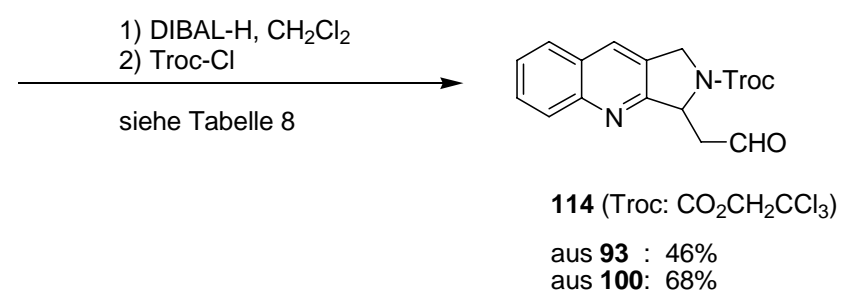

1) 1.0 Äq. DIBAL-H, $\mathrm{CH}_{2} \mathrm{Cl}_{2},-78^{\circ} \mathrm{C}, 25 \mathrm{~min}$; dann 0.51 Äq. DIBAL-H, $-78^{\circ} \mathrm{C}, 30 \mathrm{~min}$

2) $1.1 \mathrm{Äq}$. $\mathrm{ACE}-\mathrm{Cl}, \mathrm{CH}_{3} \mathrm{CN},-10^{\circ} \mathrm{C}, 1 \mathrm{~h}$; dann 1.4 Äq. ACE-Cl, $15^{\circ} \mathrm{C}, 3 \mathrm{~h}$.

54\% über zwei Stufen

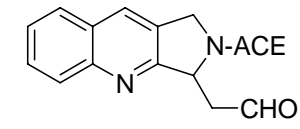

115

(ACE: $\mathrm{CO}_{2} \mathrm{CHClCH}_{3}$ )

Schema 39. Synthese von 114 und 115

\begin{tabular}{|c|c|c|}
\hline Eintrag & Reaktionsbedingungen & Ausbeute \\
\hline 1 & $\begin{array}{l}\text { 1) } 1.0 \text { Äq. 93, } 1.4 \text { Äq. DIBAL-H, Dichlormethan, }-78^{\circ} \mathrm{C} \text {, } \\
30 \text { min; } \\
\text { 2) } 1.1 \text { Äq. Troc-Cl, } 2.3 \text { Äq. } \mathrm{NaHCO}_{3} \text {, DCE, } 0{ }^{\circ} \mathrm{C}, 2.5 \mathrm{~h} \text {; dann } \\
2.8 \text { Äq. Troc-Cl, RT, } 11 \mathrm{~h}\end{array}$ & $46 \%$ \\
\hline 2 & $\begin{array}{l}\text { 1) } 1.0 \text { Äq. 100, } 1.1 \text { Äq. DIBAL-H, Dichlormethan, }-78^{\circ} \mathrm{C} \text {, } \\
40 \mathrm{~min} \text {; } \\
\text { 2) } 1.0 \text { Äq. Troc-Cl, } 0.20 \text { Äq. } \mathrm{K}_{2} \mathrm{CO}_{3} \text {, Acetonitril, }-47^{\circ} \mathrm{C}, 1 \mathrm{~h} \text {; } \\
\text { dann } 0{ }^{\circ} \mathrm{C}, 2 \mathrm{~h}\end{array}$ & $68 \%$ \\
\hline
\end{tabular}

Tabelle 8. Synthese des $N$-Troc geschützten Aldehyds 114 


\section{Durchführung der Domino-Knoevenagel-hetero-Diels-Alder- Reaktion und anschließende Bildung des Lactams}

\subsection{Domino-Knoevenagel-hetero-Diels-Alder-Reaktion bei Raum- temperatur und anschließende Lactambildung}

\subsubsection{Umsetzung des $N$-Bn-geschützten Aldehyds 94}

Zur Durchführung der Domino-Knoevenagel-hetero-Diels-Alder-Reaktion wurde der Aldehyd 94 mit 1.0 Äq. Meldrum-Säure (46), 2.0 Äq. des Enolethers 64 und einer katalytischen Menge EDDA in Toluol versetzt. Durch die dünnschichtchromatographischen Reaktionskontrolle wurde gefunden, dass der Aldehyd $94 \mathrm{sehr}$ schnell reagierte und in kurzer Zeit vollständig verbraucht wurde. Jedoch fand gleichzeitig Zersetzung statt, wobei sehr polare Substanzen in großer Menge gebildet wurden. Längere Reaktionszeiten bei Raumtemperatur führten nicht zu einer Verbesserung. Die Reaktion wurde daher nicht weiter verfolgt.

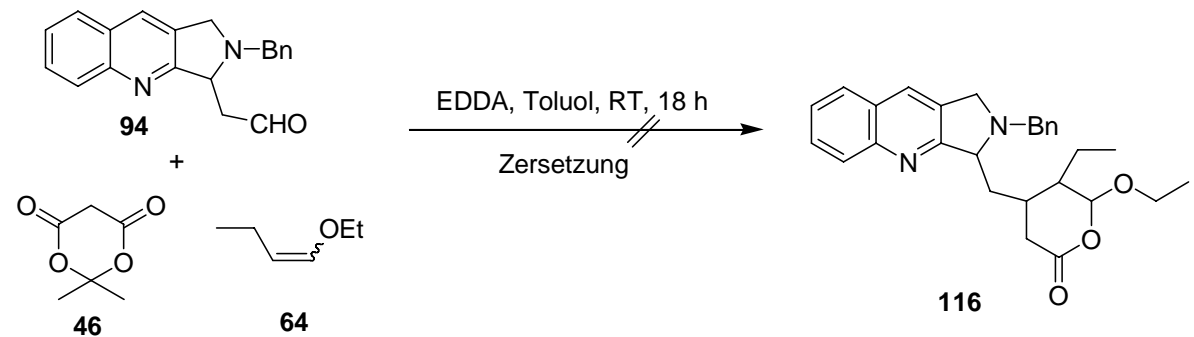

Schema 40. Versuch der Domino-Knoevenagel-hetero-Diels-Alder-Reaktion mit 94

\subsubsection{Umsetzung des $N$-Cbz-geschützten Aldehyds 96}

Zur Durchführung der Domino-Knoevenagel-hetero-Diels-Alder-Reaktion wurde der Aldehyd 96 mit Meldrum-Säure, dem Enolether 64 und einer katalytischen Menge EDDA in Toluol versetzt (siehe Tabelle 9). Das Domino-Produkt 117 konnte unabhängig von Reaktionsdauer in 50\% Ausbeute chromatographisch isoliert werden (Eintrag 1 und 2). Aber die anschließende Lactambildung durch die Cbz-Entschützung und intramolekulare Cyclisierung war problematisch. Es konnte der Aldehyd $\mathbf{6 1}$ nur in Spurenmengen detektiert werden.

Im Hinblick auf die Labilität auf Kiesegel des Dominoproduktes 117 wurden andere Versuche unter ähnlichen Bedingungen ohne chromatographische Isolierung von 117 durchgeführt 
(Eintrag 3). Trotz der hohen Rohausbeute (111\%) der Domino-Reaktion scheiterten sie an der anschließenden Lactambildung.

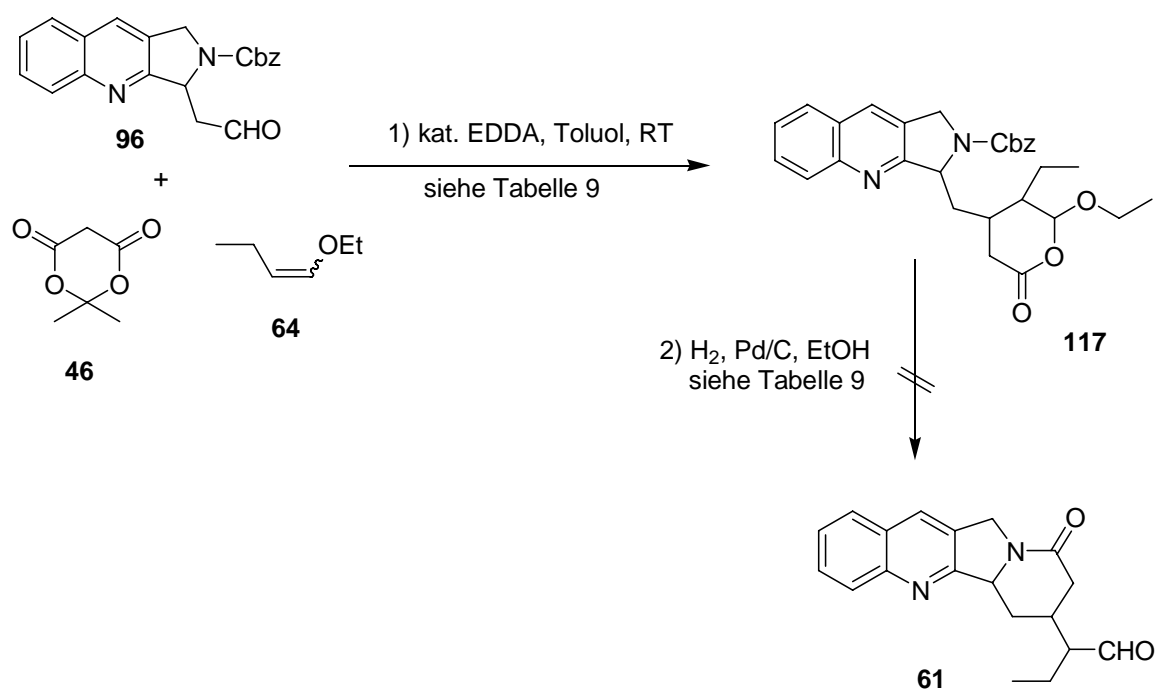

Schema 41. Versuch der Domino-Reaktion mit 96 und anschließende Lactambildung

\begin{tabular}{lcc}
\hline Eintrag & Reaktionsbedingungen & Ausbeute \\
& von $\mathbf{6 1}$
\end{tabular}

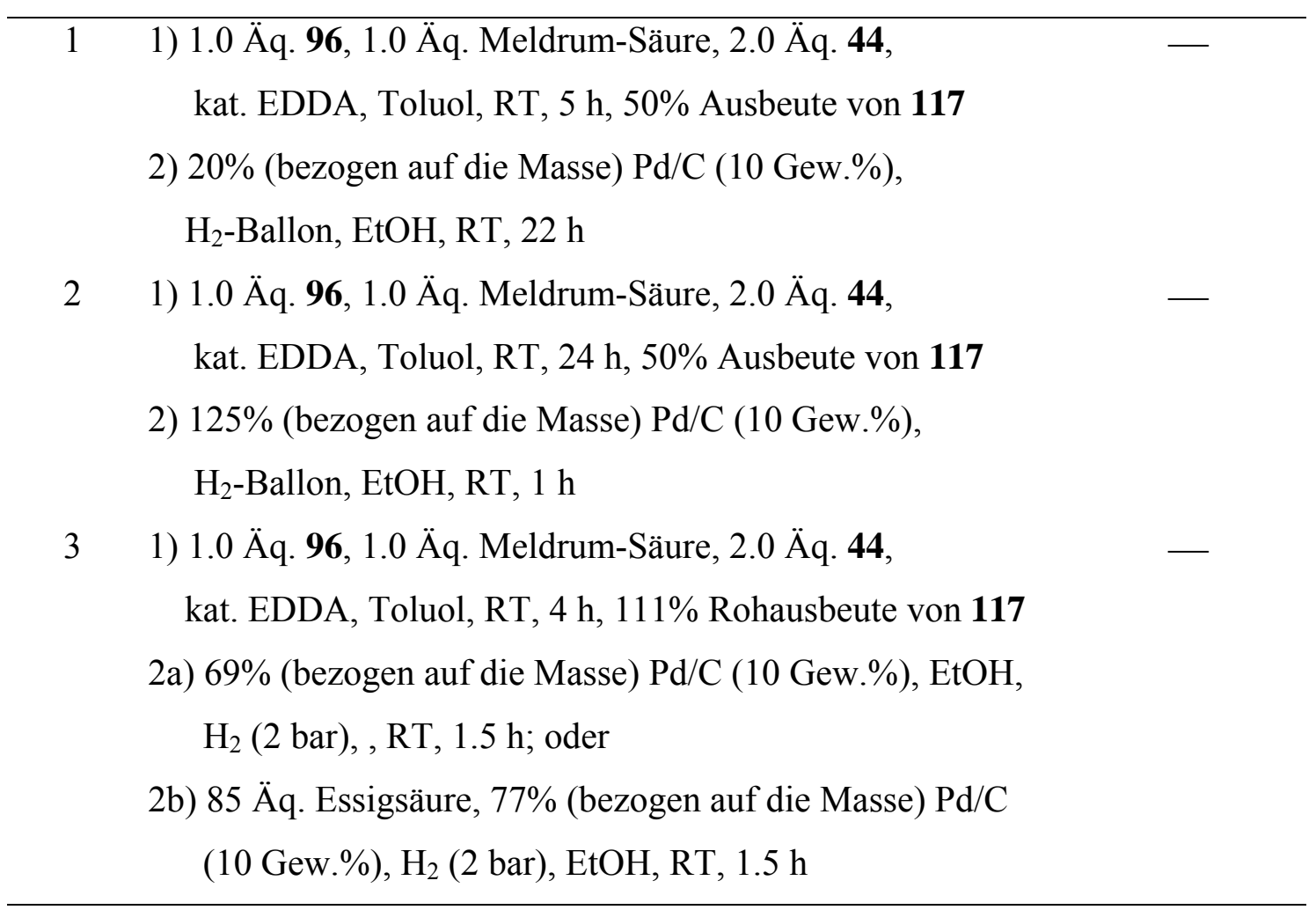

Tabelle 9. Versuch der Synthese von 61 durch Domino-Reaktion und anschließende Lactambildung. 


\subsubsection{Umsetzung des $N$-Troc-geschützten Aldehyds 114}

Der Aldehyd 114 wurde in einer Domino-Knoevenagel-hetero-Diels-Alder-Reaktion mit Meldrum-Säure und 64 umgesetzt. Nach drei Stunden Rühren in Toluol bei Raumtemperatur konnte das Rohprodukt durch einfache Konzentration gewonnen werden, welches ohne weitere Reinigung zur Troc-Entschützung mit aktiviertem Zink (Pulver) ${ }^{72}$ in Gegenwart von Essigsäure umgesetzt wurde. Neben minimaler Zersetzung konnte keine Bildung von 61 beobachtet werden. Ein Versuch der Reaktion in EtOH unter Erwärmung blieb ebenfalls erfolglos. Durch Zugabe von Wasser statt Essigsäure ließ sich 61 letztlich in einer Ausbeute von $41 \%$ über zwei Stufen dargestellt werden.

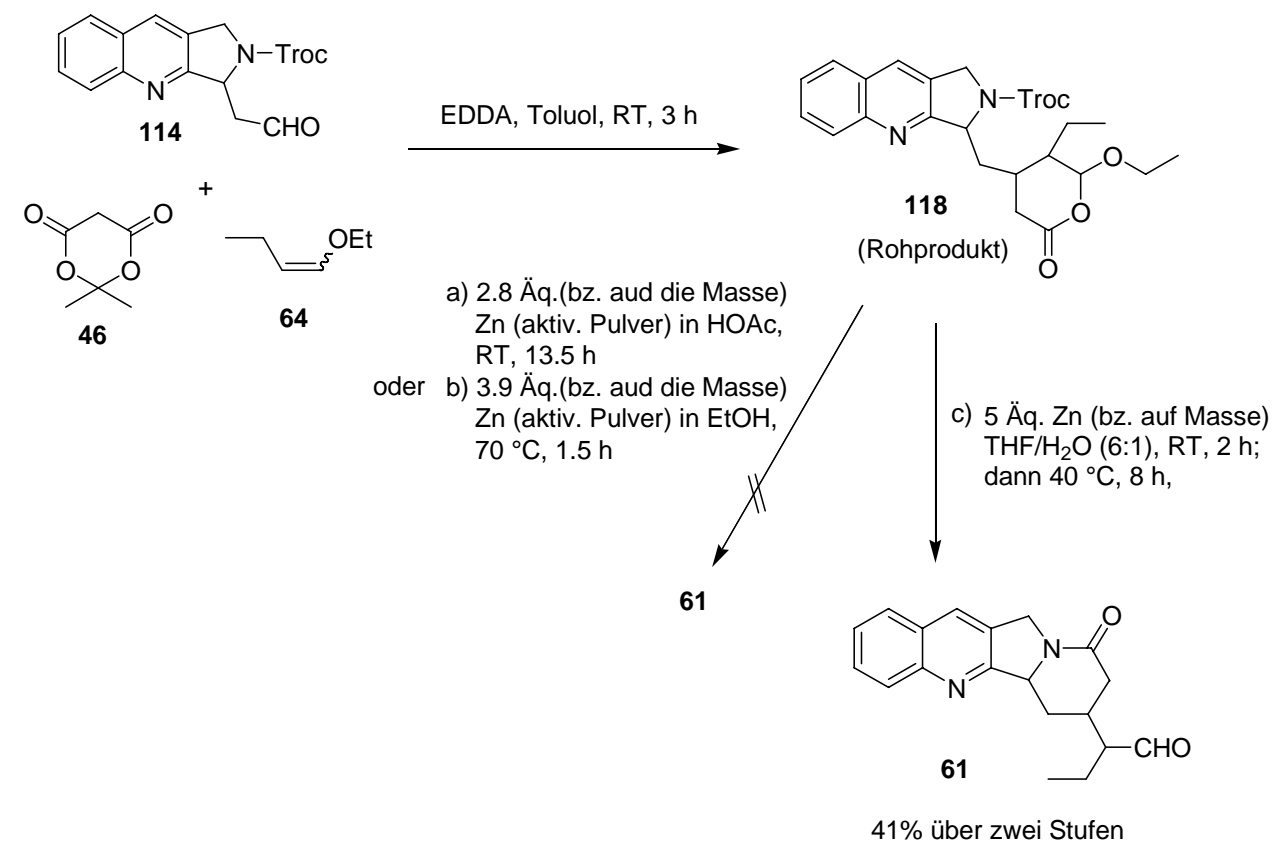

Schema 42. Synthese von 61 durch Domino-Reaktion mit 114 und anschließende Lactambildung 


\subsubsection{Umsetzung des $N$-ACE-geschützten Aldehyds 115}

Die Domino-Knoevenagel-hetero-Diels-Alder-Reaktion mit dem Aldehyd 115 wurde auf ähnliche Weise wie die obigen bei Raumtemperatur durchgeführt. Das Rohprodukt wurde direkt zurm anschließenden Lactamringaufbau verwendet. Bei der ersten Untersuchung mit 1,2-Dichlorethan als Lösungmittel konnte keine Reaktion unter Erwärmung beobachtet werden. Hingegen in Methanol $^{71}$ mit Essigsäure als Additiv oder in Ethanol mit Natriumcarbonat als Additiv fand Zersetzung sofort statt. Da 61 nicht spektroskopisch beobachtet werden konnte, wurde auf weitere Versuche verzichtet.

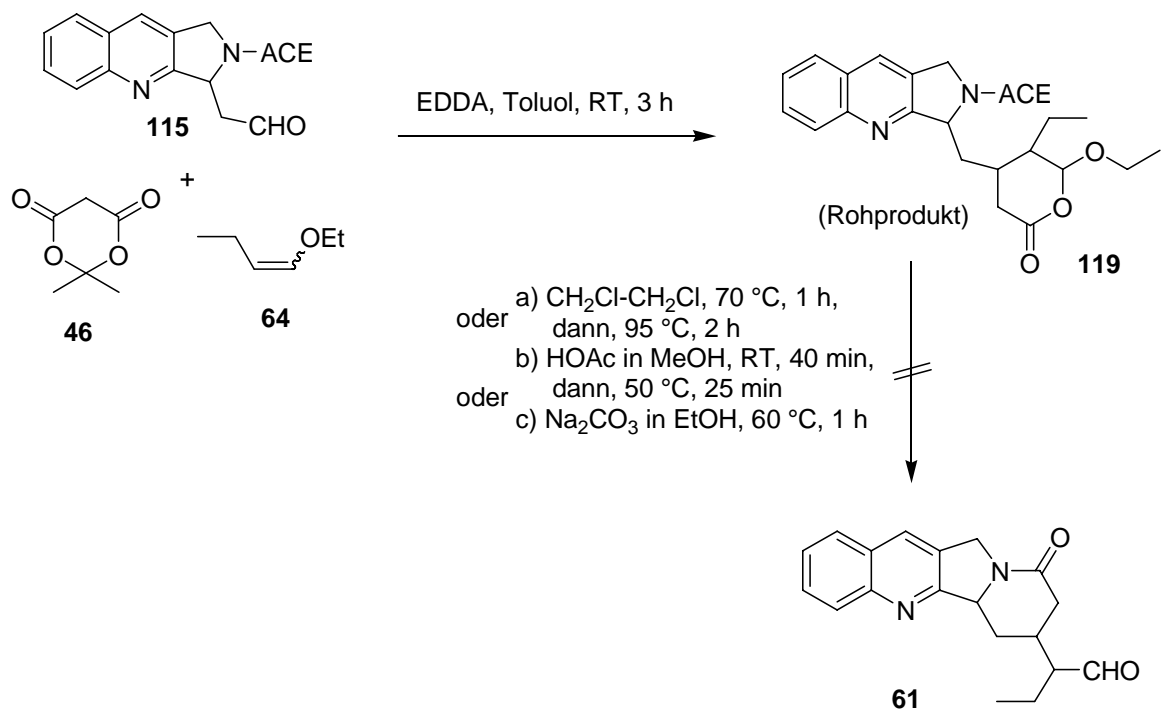

Schema 43. Versuch der Domino-Reaktion von 115 und anschließenden Lactambildung 


\subsection{Domino-Knoevenagel-hetero-Diels-Alder-Reaktion bei erhöhter Temperatur und anschließende Lactambildung}

\subsubsection{Feststellung des Lactambildungsproblems}

Das Problem bei der Synthese von 61 (siehe Kap. 3.1.2) ließ sich aufklären, nachdem ESIMassenspektren des Rohproduktes der Domino-Knoevenagel-hetero-Diels-Alder-Reaktion sorgfältig analytisiert worden waren. Neben dem gewünschten Produkt 117 konnten das Zwischenprodukt 121 in hoher sowie das Knoevenagel-Produkt 120 in geringer Intensität nachgewiesen werden.

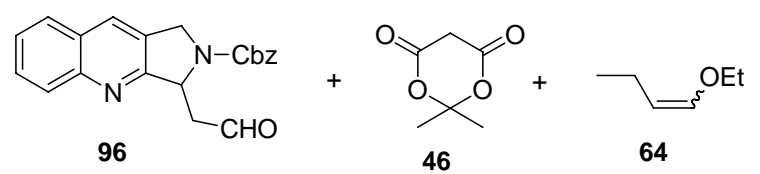

kat. EDDA, Toluol, RT<smiles>CCCCC1c2nc3ccccc3cc2CN1CCC1c2nc3ccccc3cc2CN1C(=O)c1ccccc1</smiles>

Schema 44. Produktverteilung der Domino-Knoevenagel-hetero-Diels-Alder-Reaktion bei Raumtemperatur

Es konnte die Vermutung, dass der Fehlschlag der Reaktion des Gemisches zu 61 auf Probleme bei der hydrogenolytischer Cbz-Entschützung zurückzuführen sei, durch die sehr schnell verlaufende $N$-Cbz-Entschützung von 97 mit gleicher Gerüststruktur wie 117 widerlegt werden (Schema 45).

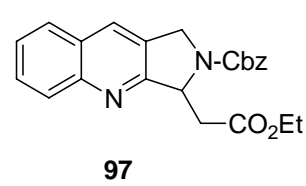

97

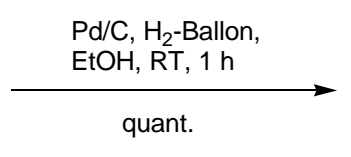

quant

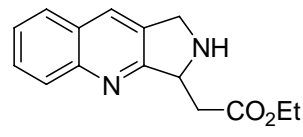

98

Schema 45. Testreaktion der Cbz-Entschützung von 97 


\subsubsection{Durchführung der Domino-Reaktion mit dem $N$-Cbz-geschützten Aldehyd 96}

Die Domino-Reaktion wurde erneut bei erhöhter Temperatur untersucht, um das gebildete Zwischenprodukt 121 sowie das Knoevenagel-Produkt 120 zum gewünschten Produkt umzusetzen. Währen das polarere Lösungsmittel Acetonitril das Produkt 117 in einer geringeren Ausbeute 67\% in drei Stunden liefern konnte, stellte Toluol das bessere Ergebnis mit 78\% Ausbeute über längere Reaktionszeit dar (siehe Tabelle 10). Die Massenanalyse des isolierten Produktes konnte das Vorhandensein von 120 und 121 ausschließen. Es war zu bemerken, dass die Domino-Reaktion in Acetonitril zwar beschleunigt wurde, aber wesentliche Zersetzung auftrat. Höhere Temperatur $\left(70-90{ }^{\circ} \mathrm{C}\right)$ führte $\mathrm{zu}$ mehr Zersetzung (Eintrag 3).

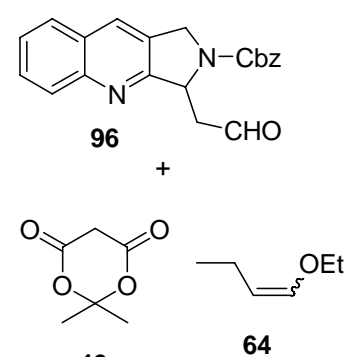

46
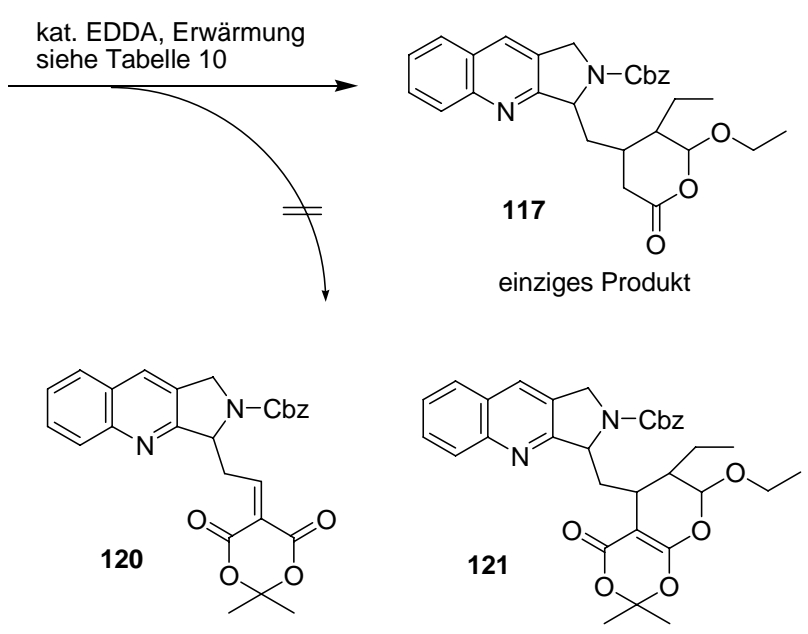

Schema 46. Domino-Reaktion mit 96 unter Erwärmung

\begin{tabular}{ccc}
\hline Eintrag & \multicolumn{1}{c}{ Reaktionsbedingungen } & Ausbeute \\
\hline 1 & 1.0 Äq. 96, 1.0 Äq. Meldrum-Säure, 5.0 Äq. 64, $12 \mathrm{~mol} \%$ & $67 \%$ \\
& EDDA, Acetonitril, $50{ }^{\circ} \mathrm{C}, 3 \mathrm{~h}$ & \\
2 & 1.0 Äq. 96, 1.0 Äq. Meldrum-Säure, 4.0 Äq. 64, $4.0 \mathrm{~mol} \%$ & $78 \%$ \\
& EDDA, Toluol, $50{ }^{\circ} \mathrm{C}, 19 \mathrm{~h}$ & \\
3 & 1.0 Äq. 96, 1.0 Äq. Meldrum-Säure, 4.2 Äq. 64, $20 \mathrm{~mol} \%$ & $28 \%$ \\
& EDDA, Toluol, $70{ }^{\circ} \mathrm{C}, 1.5 \mathrm{~h}$; dann $90^{\circ} \mathrm{C}, 3 \mathrm{~h}$ & \\
\hline
\end{tabular}

Tabelle 10. Synthese von 117 durch Domino-Reaktion unter Erwärmung 


\subsubsection{Lactambildung}

Der Aufbau des Lactamrings erfolgte durch eine zweite Domino-Reaktion bzw. CbzEntschützung und anschließende intramolekulare Lactamringschließung. Unter typischen hydrogenolytischen Bedingungen mit Ethanol als Lösungsmittel wurde 117 in $2.5 \mathrm{~h}$ in den Aldehyd 61 in 76\% Ausbeute umgesetzt. Hingegen mit EtOAc als Lösungsmittel wurde die Reaktion so verlangsamt, dass die Reatkion über Nacht laufen lassen musste. Nach siebzehn Stunden konnte $\mathbf{6 1}$ in 75\% Ausbeute isoliert werden. Es war bemerkungswert, dass die Ausbeute auf $86 \%$ erhöht werden konnte, wenn das Edukt 117 vorher zweimal chromatographisch gereinigt worden war.

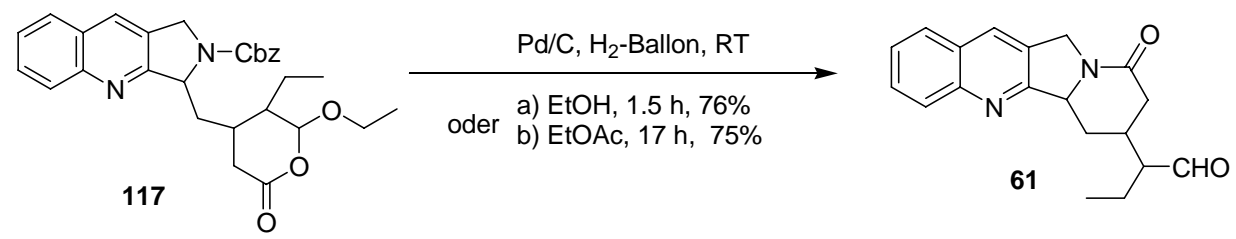

Schema 47. Synthese des Aldehyds 61

\subsection{Beweis der Domino-Reaktionen}

Da die Verbindung 61 als ein Gemisch von vier Diastereomern vorliegt, wurde auf einen genauen Strukturnachweis verzichtet. Vielmehr wurde 61 mit DDQ umgesetzt. Unter Erwärmung konnten die beiden Verbindungen 122 und 123 in 55\% bzw. 33\% Ausbeute isoliert werden. Die Bildung von 123 zeigt, dass eine Aldehydfunktionalität nicht ganz kompatible mit DDQ-vermittelter Dehydrierung ist. Die Bildung von 123 könnte auf eine Spaltung der Enolform von 122 zurückgeführt werden.
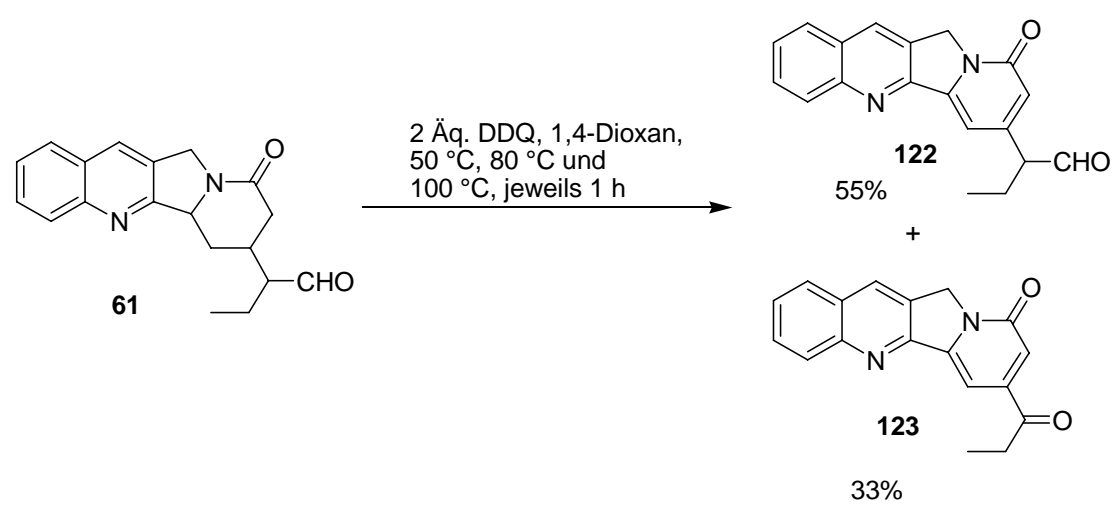

Schema 48. Beweis der Domino-Reaktionen durch Synthese von 122 


\subsection{Mechanismus der Domino-Knoevenagel-hetero-Diels-Alder-Reaktion}

Aus der obigen Ergebnisse kann der folgende Mechanismus für die Domino-Knoevenagelhetero-Diels-Alder-Reaktion unter Verwendung von 96 angenommen werden. Ähnliche Annahmen sollten auch für die Umsetzung der $N$-Troc- und $N$-ACE-geschützten Aldehyden 114 bzw. 115 gelten.

Zuerst wird durch eine Knoevenagel-Kondensation von 96 mit Meldrum-Säure das Oxa-1,3butadien 120 gebildet (siehe Schema 49). Die anschließende hetero-Diels-Alder-Reaktion mit 64 liefert das Zwischenprodukt 121. Es konnte nachgewiesen werden, dass 120 bei Raumtemperatur noch in einem geringen Anteil vorlag. Des weiteren stehen 121 zwei weitere Reaktionswege offen. Bei einem (Weg A) wird 121 durch Freisetzung von Aceton in das Keten 127 überführt. In einer folgenden Addition von Wasser, das bei der KnoevenagelKondensation gebildet wurde, wird die $\beta$-Keton-carbonsäure 128 geliefert, welche zum Domino-Produkt 117 decarboxyliert. 128 kann auch durch einen direkten Wasserangriff auf das reaktive Kohlenstoffzentrum (Weg B) von 121 gebildet werden. Beide Reaktionswege haben hierbei sicherlich den größeren Energiebedarf. Bei Raumtemperatur scheinen die Reaktionswege A und B nicht durchlaufen werden, wie die schlechte Ausbeuten der DominoKnoevenagel-hetero-Diels-Alder-Reaktion bei Raumtemperatur zeigen. Erst bei höheren Temperaturen erfolgt die schnelle Umsetzung von 121 zu 117.

Die Öffnung des 1,3-Dioxanringes kann auch durch Umsetzung in Gegenwart von Methanol erfolgen, wie von $H$. Denzer ${ }^{73}$ beobachtet wurde. Dies wird hier für das $N$-Troc-geschützten Produkt 125 gezeigt (siehe Schema 50). Dabei wurde die eigentliche Domino-Reaktion in drei separaten Stufen durchgeführt. Zuerst wurde Meldrum-Säure mit dem Aldehyd 114 in Toluol unter trockenen Bedingungen umgesetzt. Nach Entfernung des Trockenmittels wurde das Dienophil 64 eingesetzt. Letztlich wurde Methanol zugegeben. Durch die Massenanalyse des Rohproduktes ließen sich zwei Verbindungen 118 und 126 nachweisen. Während die Bildung von 126 durch Methanolyse von 125 erklärt werden kann, zeigt die Bildung von 118, dass bei $50{ }^{\circ} \mathrm{C}$ auch eine direkte Abspaltung von Aceton und CO zu 118 aus 125 möglich ist. Es muss hier aber darauf hingewiesen werden, dass die Bildung von 118 aus 125 auch auf geringe Mengen an Wasser in Reaktionsmedien zurückzuführen sein könnte, die trotz sehr sorgfältiger Reaktionsführung nicht ganz auszuschließen sind. 

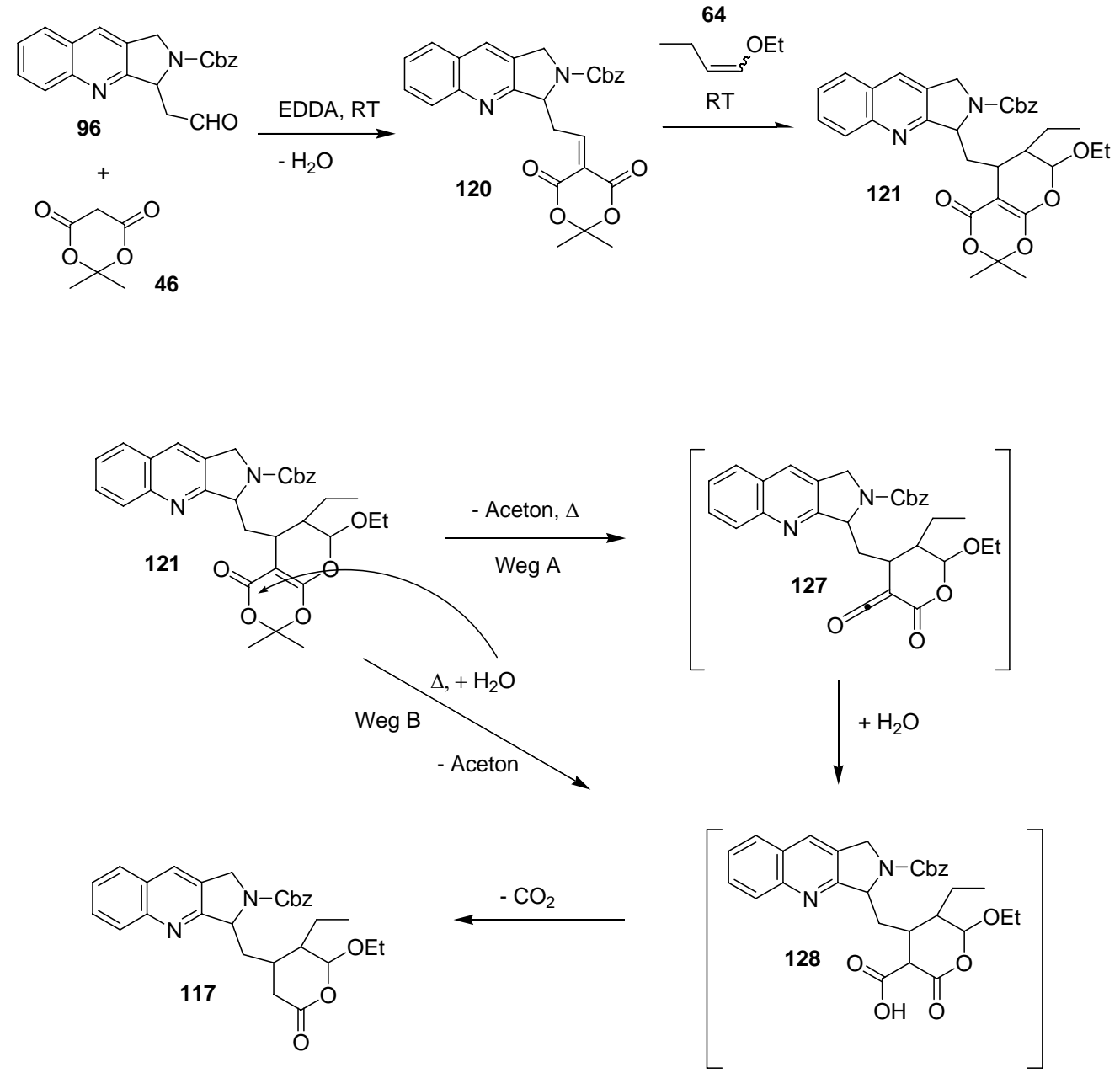

Schema 49. Mechanismus der Domino-Knoevenagel-hetero-Diels-Alder-Reaktion
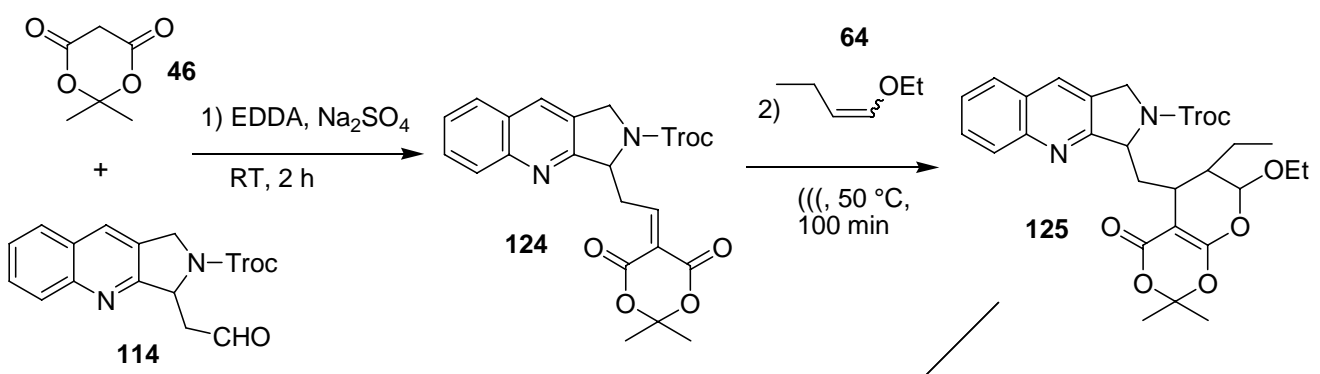

3) $\mathrm{MeOH},\left(\left(\left(, 50^{\circ} \mathrm{C}\right.\right.\right.$, $140 \mathrm{~min}$
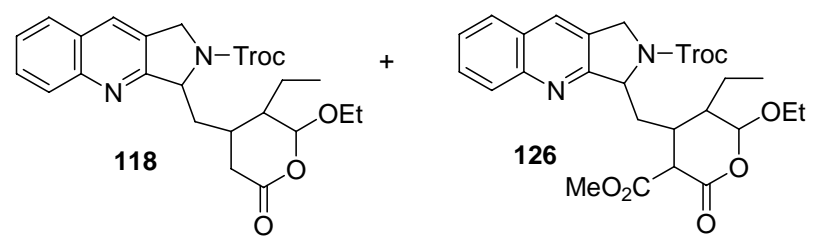

$118: 126=9.8: 1$

Schema 50. Separate Umsetzungen zur Untersuchung der Domino-Reaktion 


\section{Aufbau des Lactonrings und Synthese von Camptothecin}

Basierend auf der bereits literaturbekannten Einführung der Hydroxygruppe am Lactonring ${ }^{74}$ sollte Camptothecin durch Bildung des Lactons vollendet werden. Der Aufbau des Lactonrings musste durch Einführung einer Funktionalität an der 3-Position des Lactamrings von 61 erfolgen. Ausgehend von einer Domino-Knoevenagel-hetero-Diels-Alder-Reaktion ergaben sich dann zur Funktionalisierung zwei Ausgangspunkte: Lactam-Derivate von 61 und Domino-Produkte (117 oder 118).

\subsection{Untersuchung zur Funktionalisierung des Lactamrings}

\subsubsection{Aldol-Reaktion der Carbonsäure 129 und des Methylesters 132}

Die effektivste Methode zur Einführung einer Hydroxymethylgruppe ist eine Aldolreaktion der Carbonsäure 129 mit Formaldehyd. Dazu wurde der Aldehyd 61 erst unter Verwendung von $\mathrm{NaClO}_{2}{ }^{75}$ zur 129 in $90 \%$ Ausbeute oxidiert. Die anschließende Aldolreaktion wurde sowohl mit durch Thermolyse hergestelltem, gasförmigem Formaldehyd als auch mit frisch hergestellter Formaldehyd-Lösung ${ }^{76}$ durchgeführt. Für die Enolatbildung des Amids von 129 wurden KHMDS, LiHMDS und LDA als Base eingesetzt (siehe Tabelle 11). Die jeweilige Massenanalyse des Rohproduktes konnte weder 130 noch 131 noch bestätigen.

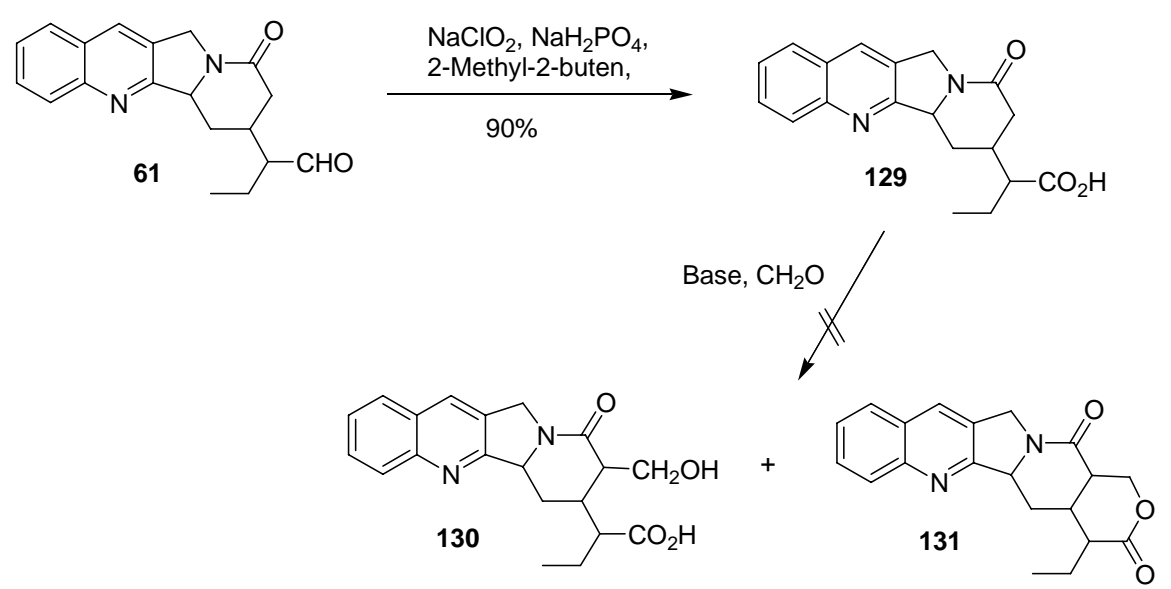




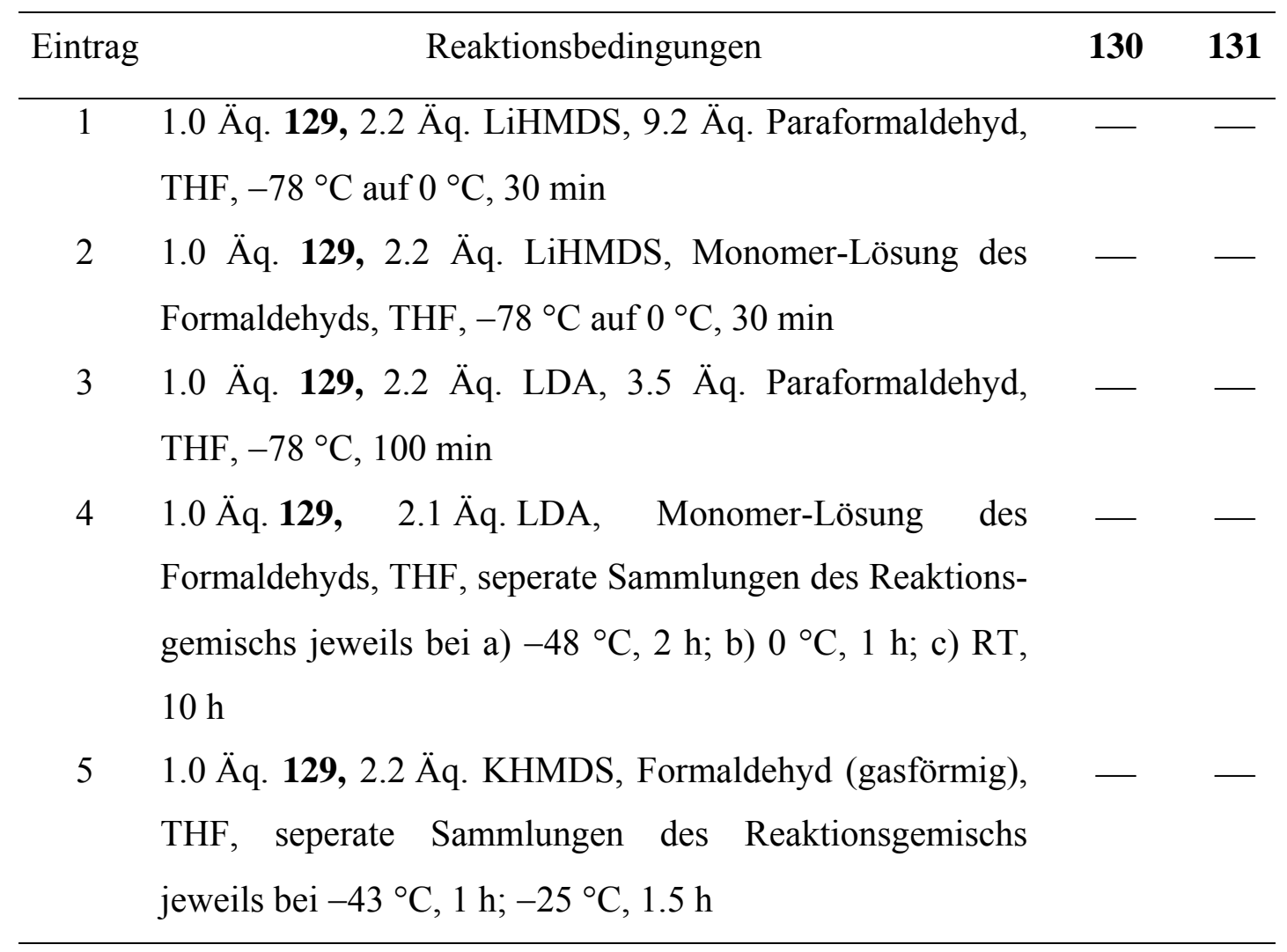

Tabelle 11. Untersuchung der Aldol-Reaktion der Carbonsäure 129

Da die vorhandene Carboxylgruppe die Enloatbildung verhindern konnte, wurde die Carbonsäure 129 in den Methylester 132 überführt. Die Veresterung wurde mit TMS-CHN ${ }^{77}$ durchgeführt, was eine gute Alternative zum gängigen Diazomethan darstellt. Der Ester 132 konnte unter Standard-Bedingungen in 93\% Ausbeute erhalten werden, wobei die Bildung des Silylesters 133 als Konkurrenzreaktion nicht auftrat. Während mit KHMDS oder LDA in stöchiometrischer Menge neben geringer Zersetzung nur das Edukt zurückgewonnen werden konnte, führte ein Überschuss an Base zur Zersetzung (siehe Tabelle 12). 


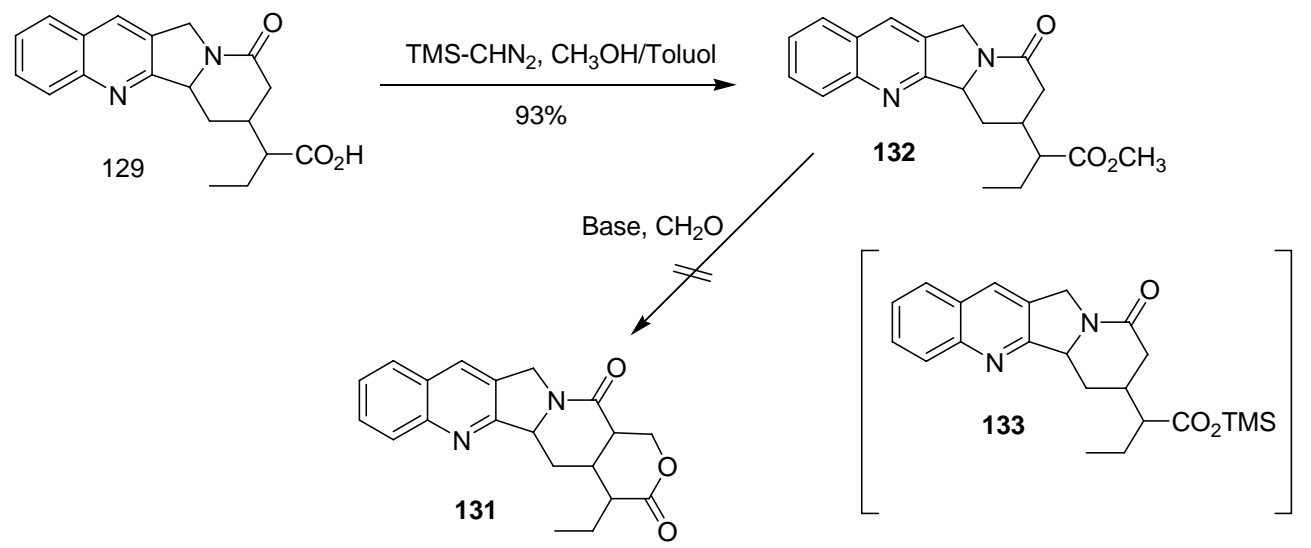

Schema 52. Untersuchung der Aldol-Reaktion des Methylesters 132

\begin{tabular}{|c|c|c|}
\hline Eintrag & Reaktionsbedingungen & Aubeute \\
\hline 1 & $\begin{array}{l}\text { 1.0 Äq. 132, 1.1 Äq. KHMDS, Formaldehyd (gasförmig) in } \\
\text { THF, }-45^{\circ} \mathrm{C} \text { auf RT, } 40 \mathrm{~min}\end{array}$ & kein Umsatz \\
\hline 2 & $\begin{array}{l}1.0 \text { Äq. 132, } 1.1 \text { Äq. LDA, Formaldehyd (gasförmig) in THF, } \\
-78^{\circ} \mathrm{C} \text { auf RT, } 2 \mathrm{~h}\end{array}$ & kein Umsatz \\
\hline 3 & $\begin{array}{l}1.0 \text { Äq. 132, } 3.5 \text { Äq. LDA, Formaldehyd (gasförmig) in THF, } \\
-78^{\circ} \mathrm{C}, 2 \mathrm{~h} \text {; dann } 0{ }^{\circ} \mathrm{C}, 10 \mathrm{~min}\end{array}$ & Zersetzung \\
\hline 4 & $\begin{array}{l}1.0 \text { Äq. 132, } 2.5 \text { Äq. LDA, Formaldehyd (gasförmig) in THF, } \\
-78^{\circ} \mathrm{C}, 1.5 \mathrm{~h}\end{array}$ & Zersetzung \\
\hline
\end{tabular}

Tabelle 12. Untersuchung der Aldol-Reaktion des Methylesters 132

\subsubsection{Alkylierung des Acetals 134 mit Chlormethylbenzylether}

Um Nebenreaktionen des Aldehyds zu vermeiden, wurde die Aldehydgruppe in $\mathbf{6 1}$ in ein Acetal überführt. Das Acetal 134 konnte mit $\mathrm{RuCl}_{3}$ als Katalysator unter Erwärmung in 54\% Ausbeute dargestellt werden, wobei gleichzeitig die Einführung einer Doppelbindung stattfand. Da sich eine Benzyloxymethylgruppe durch Abspaltung der Benzylgruppe in eine Hydroxymethylguppe überführen lässt, wurde eine Alkylierung des Acetals mit Chlormethylbenzylether durchgeführt. Die Umsetzung des Acetals 134 mit LDA bei $-78^{\circ} \mathrm{C}$ führte allerdings hauptsächlich zur Zersetzung des Eduktes. Nach der Zugabe von Chlormethylbenzylether konnte das Produkt 135 in 8\% Ausbeute isoliert und das Edukt 134 in $29 \%$ Ausbeute zurückgewonnen werden. Mit KHMDS wurde keine Verbesserung beobachtet. Wegen der geringen Ausbeute wurde dieser Ansatz nicht weiter verfolgt. 


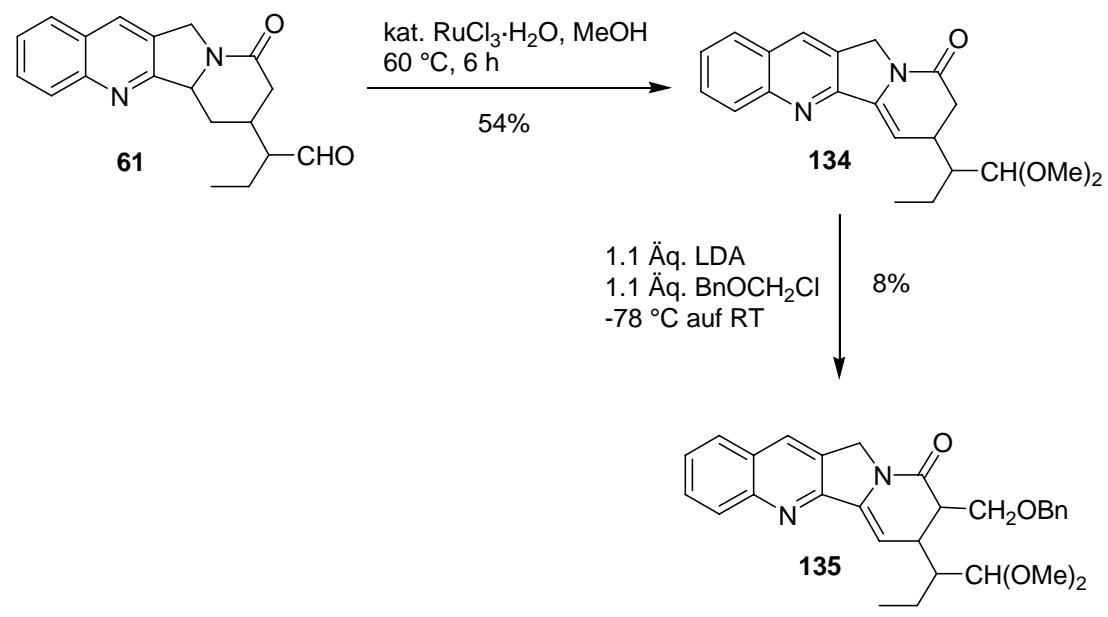

Schema 53. Alkylierung des Acetals 134

\subsubsection{Formylierung mit Bedereck-Reagenz}

Da Formylierung eine gute Alternative zu Hydroxymethylierung ist, wurde BedereckReagen $^{78}$ (t-Butoxy- $N, N, N^{\prime}, N^{\prime}$-tetramethyl-methandiamin) mit 132 umgesetzt. Trotz Erhitzen der Reaktion auf $100{ }^{\circ} \mathrm{C}$ für fünfzehn Stunden war die Bildung des Enamins 136 nicht zu beobachten, welches durch Hydrolyse den Aldehyd 137 liefern sollte.
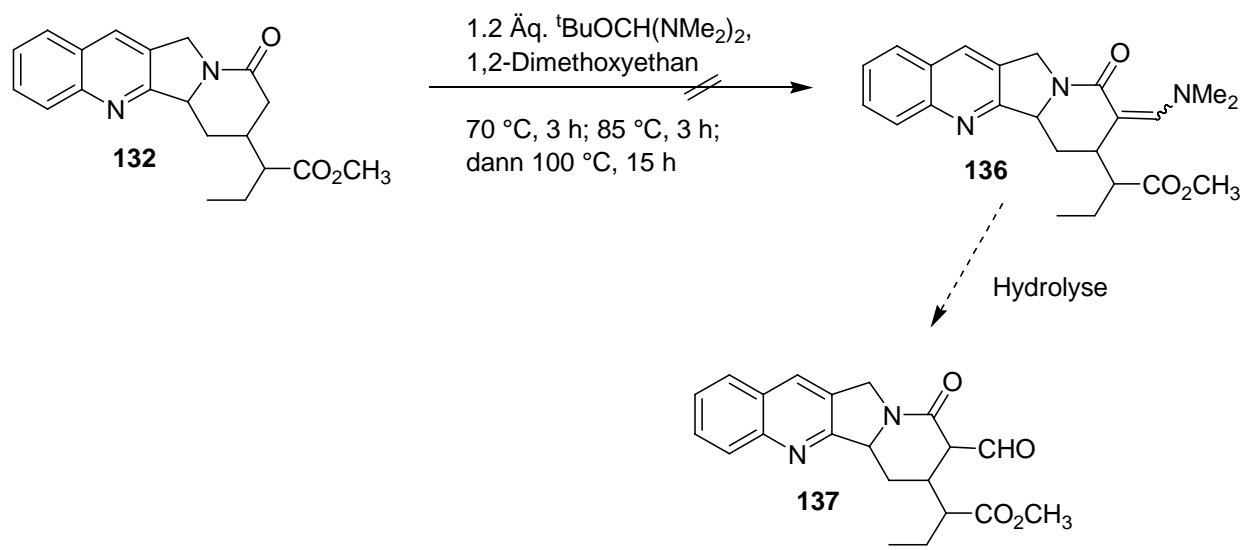

Schema 54. Formylierungsversuch von 132 mit Bedereck-Reagenz 


\subsection{Untersuchung zur Funktionalisierung des Domino-Produktes}

\subsubsection{Aldol-Reaktion von 117}

Das Domino-Produkt 117 wurde zuerst mit KHMDS umgesetzt. Dann wurde gasförmiger Formaldehyd bei tiefer Tempeartur $\left(-78^{\circ} \mathrm{C}\right.$ und $\left.-45^{\circ} \mathrm{C}\right)$ langsam eingeleitet. Eine Massenanalyse des Rohproduktes zeigte, dass das gewünschte Produkt 138 nicht gebildet wurde, sondern Zersetzung auftrat.
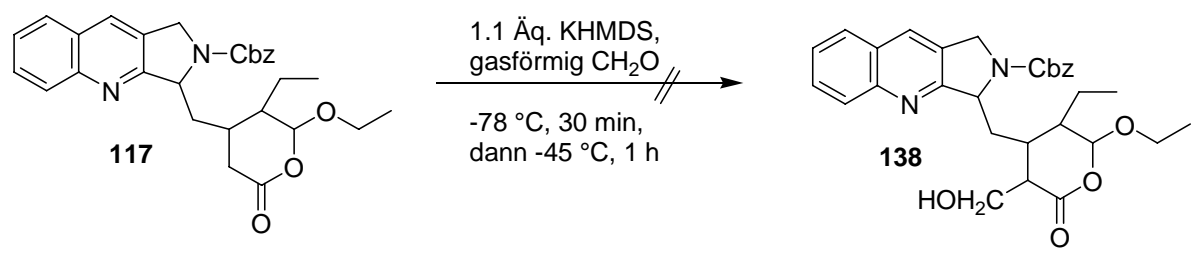

Schema 55. Versuch der Aldol-Reaktion von 117

\subsubsection{Alkylierung von 117 mit Chlormethylbenzylether}

Die Alkylierung von 117 mit Chlormethylbenzylether sollte ebenfalls über ein intermediäres Enolat erfolgen. Da die Enolatbildung den Lactonring zerstören konnte, wurde eine Optimierung der Zugabe durchgeführt. Dabei wurde eine Lösung von 117 und Chlormethylbenzylether langsam zu KHMDS-Lösung bei $-35^{\circ} \mathrm{C}$ gegeben, anschließend ließ man die Reaktionsmischung bei erhöhter Temperatur reagieren. Es konnte zwar das Produkt 139 durch hochgelöste Massenanalyse bestätigt werden, die chromatographisch Reinigung lieferte aber nur eine Ausbeute von $<10 \%$. In einem anderen Versuch unter gleichen Bedingungen wurde auf die chromatographische Reinigung von 139 verzichtet und das Rohprodukt wurde direkt zur hydrogenolytischen Cbz-Entschützung eingesetzt. Dabei ließ sich aber kein definiertes Produkt erhalten.
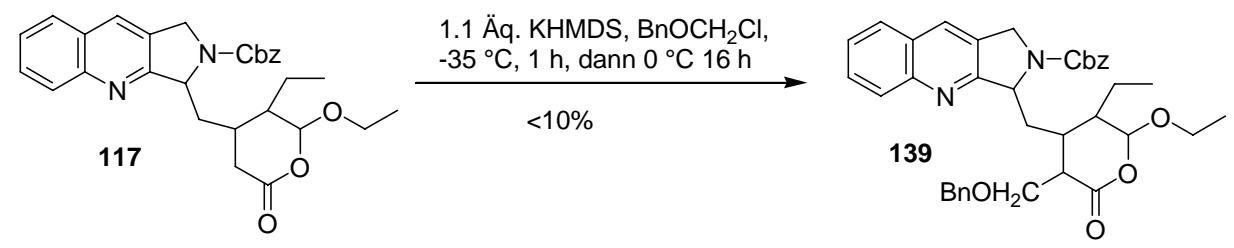

Schema 56. Alkylierung von 117 mit Chlormethylbenzylether 


\subsubsection{Formylierung von 118 mit Ameisensäureethylester}

Um die Verbindung 118 in reiner Form zu gewinnen, wurden 114, 46 und 64 in einer Domino-Knoevenagel-hetero-Diels-Alder-Reaktion für fünf Stunden unter Ultraschall bei $60{ }^{\circ} \mathrm{C}$ umgesetzt. 118 konnte in 65\% Ausbeute als Gemisch von Diastereomeren isoliert werden. Für die Enolatbildung wurde 118 zuerst mit LDA umgesetzt, dann erfolgte die Zugabe von Ameisensäureethylester ${ }^{79}$ als Formylierungsreagenz. Die Massenanalyse des Gemisches zeigte jedoch, dass eine Reaktion zu 140 nicht stattgefunden hat. Bei Verwendung von $\mathrm{NaOEt}$ als Base wurde die Bildung von 140 ebenfalls nicht beobachtet.

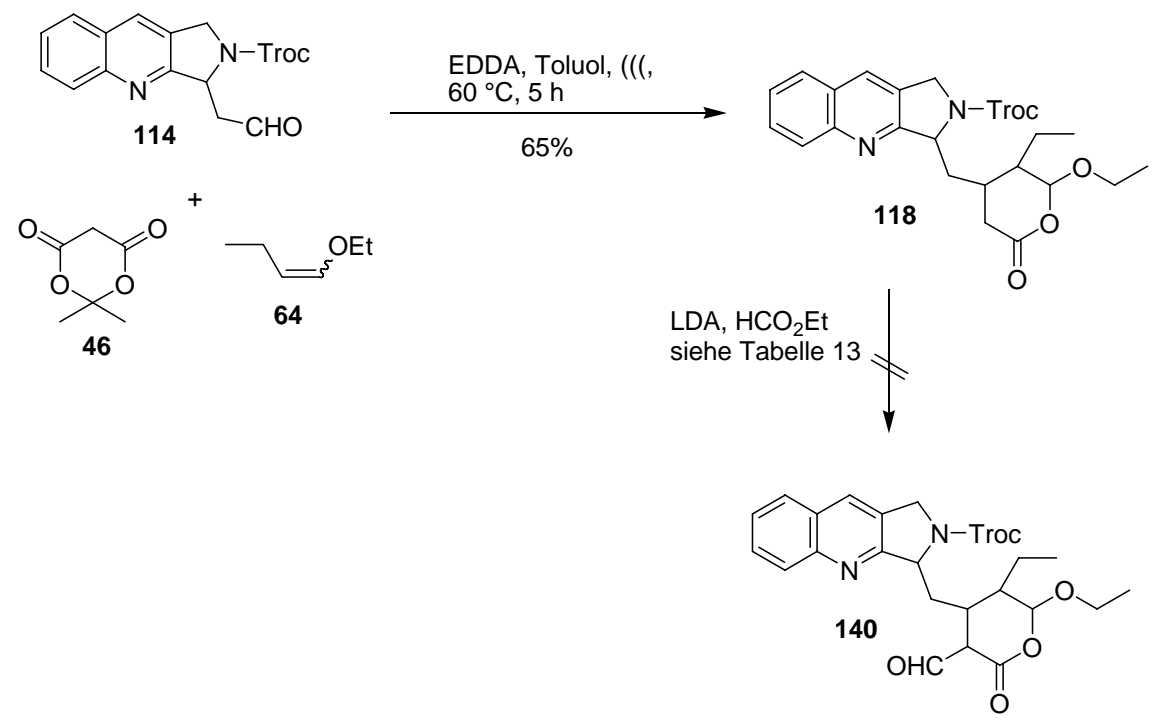

Schema 57. Formylierungsversuch von 118 mit Ameisensäureethylester

\begin{tabular}{clc}
\hline Eintrag & \multicolumn{1}{c}{ Reaktionsbedingungen } & Ausbeute \\
\hline 1 & 1.0 Äq. 118, 2.5 Äq. LDA, 8.0 Äq. Ameisensäureethylester, & - \\
& $\mathrm{THF},-78{ }^{\circ} \mathrm{C}, 30 \mathrm{~min}$, dann RT, $2 \mathrm{~h}$ & \\
2 & 1.0 Äq. 118, 4.6 Äq. NaOEt, 8.0 Äq. Ameisensäureethylester, & - \\
& $\mathrm{Et}_{2} \mathrm{O}, 0{ }^{\circ} \mathrm{C}$ auf RT, $2 \mathrm{~h}$
\end{tabular}

Tabelle 13. Versuch der Synthese von 140 


\subsection{Lactonisierung durch Danishefsky-Methode}

Für die Lactonisierung nach S. Danishefsky ${ }^{80}$ sollte zuerst der Methylester 20 dargestellt werden. Ausgehend von 61 konnte 20 in insgesamt 78\% Ausbeute über drei Stufen hergestellt werden. Dabei lieferte die Umsetzung von 132 mit DDQ unter Erwärmung auf $60{ }^{\circ} \mathrm{C}$ das gewünschte Produkt 20 in hoher Ausbeute von 93\% liefern.
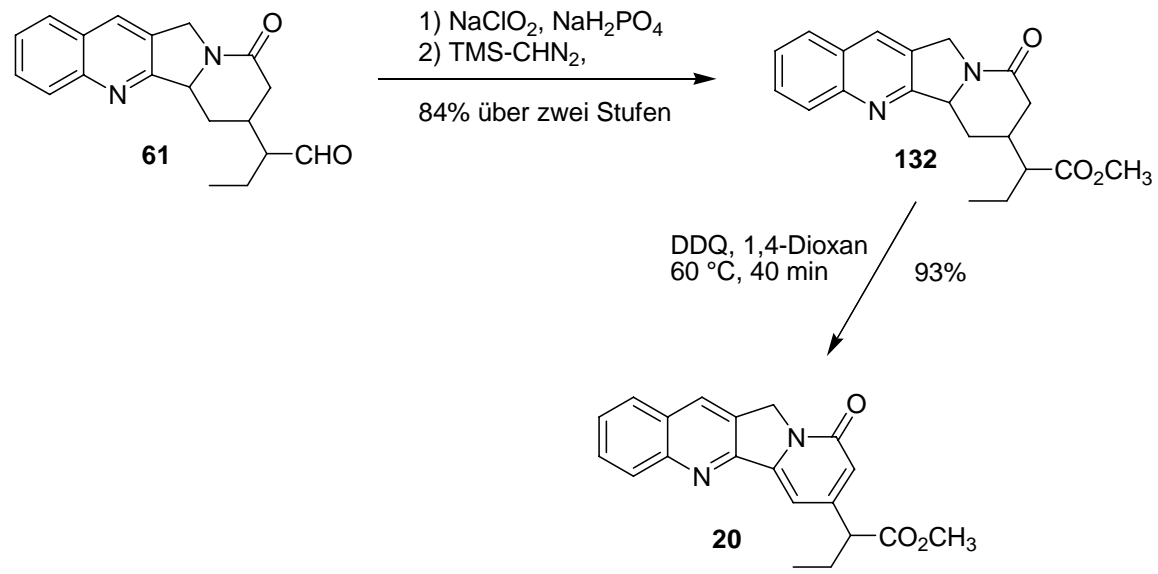

Schema 58. Synthese von 20

Die anschließende Bildung des Lactonringes unter Einführung einer $\mathrm{C}_{1}$-Einheit wurde unter den von S. Danishefsky beschriebenen Bedingungen bezüglich Konzentration, Temperatur und Reaktionszeit durchgeführt. Hierzu wurde eine Mischung von 20, Paraformaldehyd, Wasser und konz. Schwefelsäure siebzehn Stunden in einem Druckkolben erwärmt. Eine Reaktionskontrolle mittels Dünnschichtchromatographie zeigte die Bildung mehrerer Nebenprodukte. Das gewünschte Produkt 141 konnte nach chromatographischer Reinigung in $24 \%$ Ausbeute erhalten werden. Ähnliche Ausbeuten wurden schon berichtet. ${ }^{80}$ Das Spektrum des literaturbekannten Beispiels wies ebenfalls Verunreinigungen auf, welche nach einmaliger chromatographischer Reinigung noch zu beobachten waren. Wiederholte chromatographische Reinigung lieferte das gewünschte Produkt 141 in 9\% Ausbeute, wobei auch die Bildung von 142 und 143 in 7\% bzw. 38\% Ausbeute beobachtet werden konnte. 


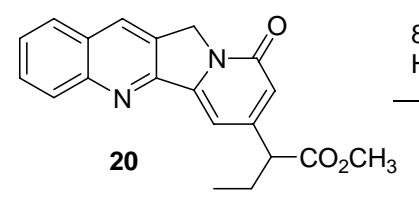

8.0 Äq. $\left(\mathrm{CH}_{2} \mathrm{O}\right)_{n}$, konz. $\mathrm{H}_{2} \mathrm{SO}_{4}$, $\mathrm{H}_{2} \mathrm{O}, 1,4$-Dioxan, $96^{\circ} \mathrm{C}, 17 \mathrm{~h}$
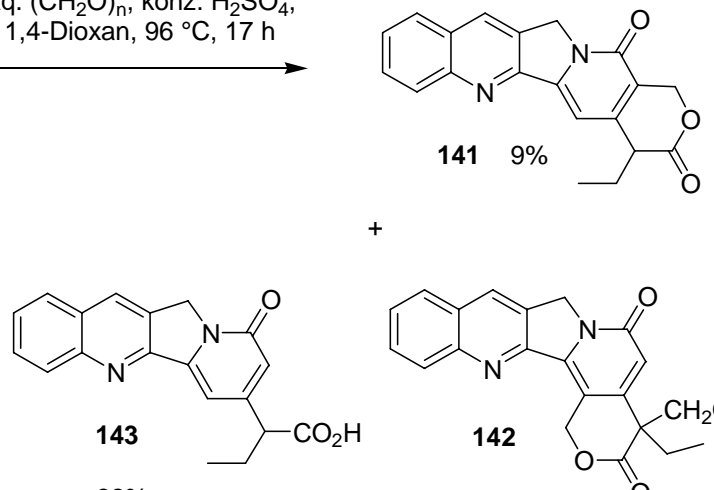

$38 \%$

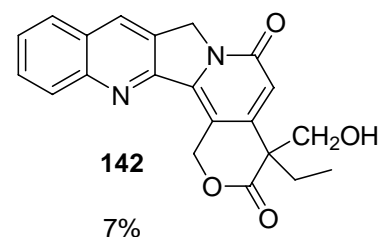

Schema 59. Synthese von 141 durch Danishefsky-Methode

Da eine Variation der Reaktionsbedingungen dieser Transformation keine Verbesserungen der Ausbeute lieferte, ${ }^{81}$ wurde als alternativ Syntheseroute einer modifizierten MannichReaktion $^{82}$ erwogen. Das Edukt 20 wurde mit Paraformaldehyd, Essigsäure und Salzsäure (34\%) in einem Druckkolben umgesetzt. Nach sechzehn Stunden bei $80{ }^{\circ} \mathrm{C}$ wurden neben 20\% des Eduktes keine definierten Produkte erhalten, der Syntheseansatz wurde daher nicht weiter verfolgt.
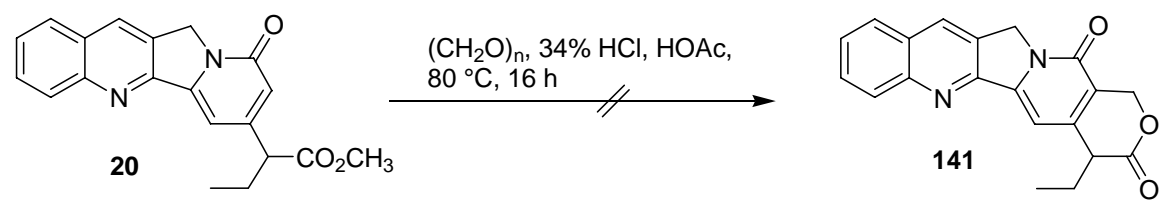

Schema 60. Versuch der Synthese von 141 durch modifizierte Mannich-Reaktion 


\subsection{Synthese von ( \pm )-Camptothecin}

Die zur Synthese von Camptothecin erforderliche Hydroxylierung am Lactonring wurde nach Winterfeldt $^{83}$ durchgeführt. 141 wurde mit Kupfer(II)-chlorid und wässriger DimethylaminLösung in DMF umgesetzt. Nach Einleitung von $\mathrm{O}_{2}$ für sieben Stunden konnte racemisches Camptothecin in $42 \%$ Ausbeute isoliert werden. Aufgrund der Hydrolyseempfindlichkeit des Lactonrings wurde die Aufarbeitung bei $\mathrm{pH}=4$ durchgeführt. Die Ausbeuten dieser literaturbekannten Umsetzung variieren zwischen $65 \%$ und $100 \%{ }^{74}$
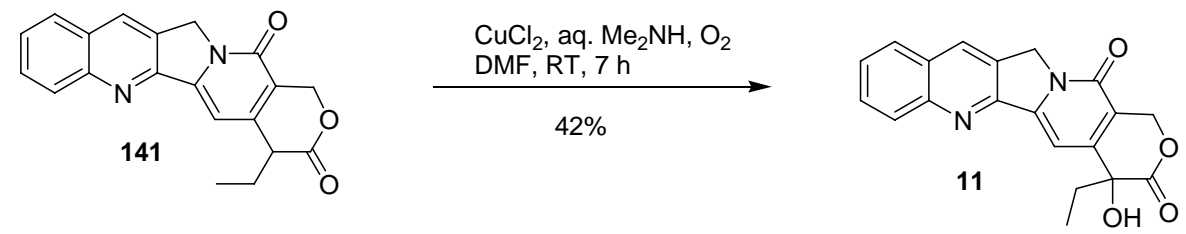

Schema 61. Synthese von ( \pm -Camptothecin 11

\subsubsection{Diskussion ausgewählter spektroskopischer Daten von $( \pm)$-Camptothecin}

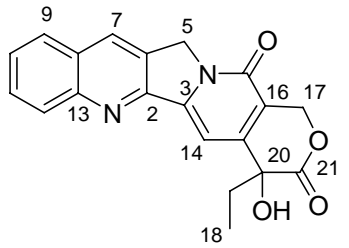

Das ${ }^{1} \mathrm{H}-\mathrm{NMR}-$ Spektrum von ( \pm )-Camptothecin (siehe Abbildung 10) zeigt im Tieffeld die für die Chinolin-Einheit charakteristischen Signale. Durch Vergleich mit Spektren seiner Vorläufer z. B. des Esters 100 lassen sich alle aromatischen Protonen zuordnen, wobei das Singulett bei $\delta=8.39$ dem Proton an C-7 entspricht. $12-\mathrm{H}$ und 9-H sind jeweils als Dublett bei $\delta=8.24$ bzw. 7.94 zu finden. 11-H resoniert als zentriertes Multiplett bei $\delta=7.83$, und 10-H sowie 14-H als Multipletts bei $\delta=7.63-7.70$. Dem einen diastereotopen Proton an C-17 ist ein Dublett bei $\delta=5.78$ mit einer großen geminalen Kopplungskonstante von $J=16.2 \mathrm{~Hz}$ zuzuordnen. Das Signal für das andere diastereotope Proton findet man gemeinsam mit den Signalen für diese zwei Protonen an C-5 in Form eines Multipletts bei $\delta=5.28-5.35$. Die HAtome der restlichen Methylen- und Methylgruppe resonieren als zentriertes Multplett bei $\delta=$ 1.90 bzw. als Triplett bei $\delta=1.04$. 
Das IR-Spektrum weist eine Bande bei $\widetilde{v}=3277 \mathrm{~cm}^{-1}$ für eine O-H Streckschwingung auf. Außerdem sind die Banden bei $\widetilde{v}=1755$ und $1651 \mathrm{~cm}^{-1}$ auf $\mathrm{C}=\mathrm{O}$ Streckschwingungen zurückzuführen. Das hoch aufgelöste ESI-Massenspektrum weist ein Signal bei $\mathrm{m} / \mathrm{z}=$ 349.1183 für $[\mathrm{M}+\mathrm{H}]^{+}$(Ber. $\left.m / z=349.1182\right)$ auf.
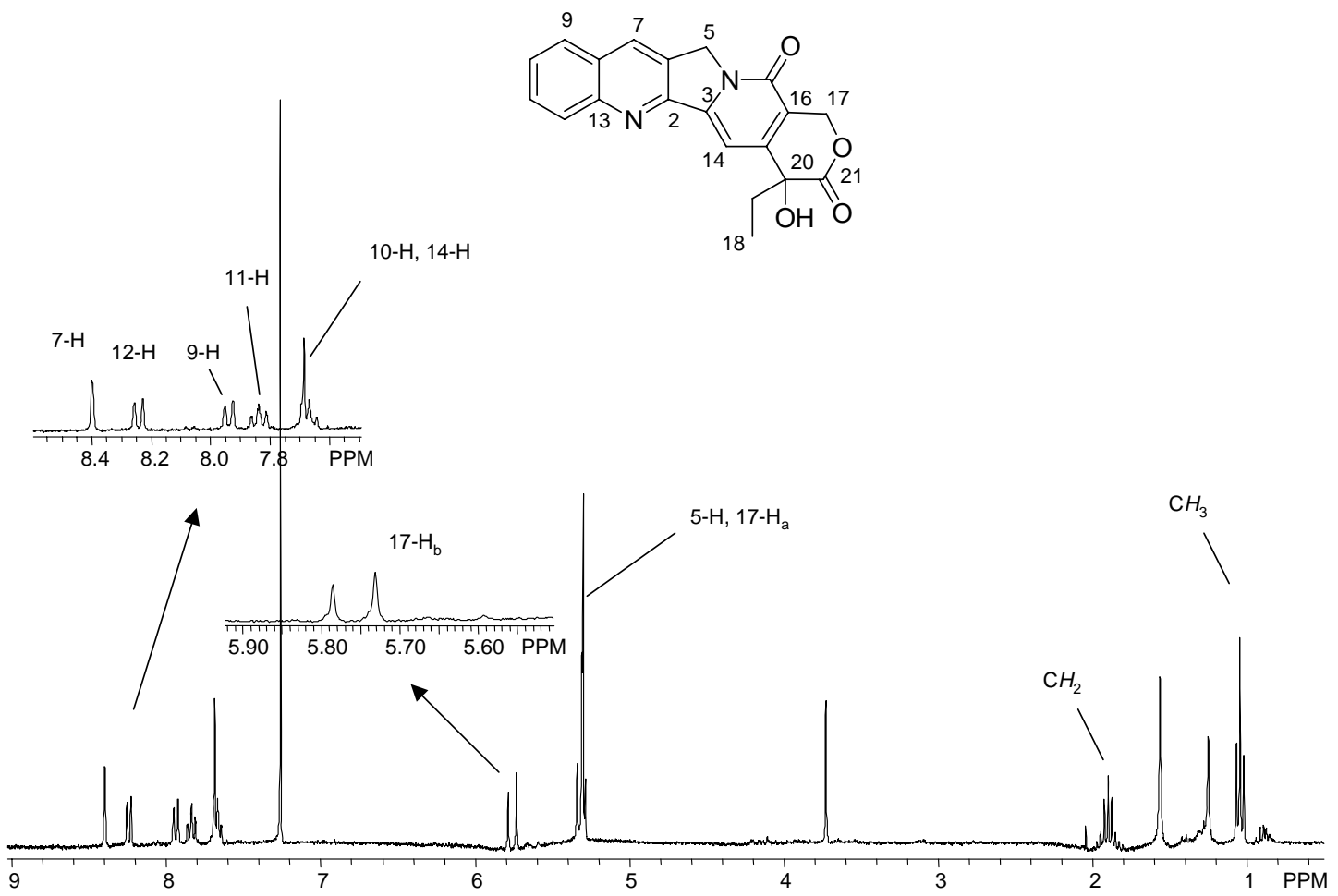

Abbildung 10. ${ }^{1} \mathrm{H}-\mathrm{NMR}\left(\mathrm{CDCl}_{3}, 300 \mathrm{M}\right)$ von $( \pm)$-Camptothecin 


\subsection{Untersuchung zur Synthese von (20S)-Camptothecin}

Da Camptothecin in der Natur als enantiomerenreines (20S)-Camptothecin vorliegt und nur dieses Enatiomer biologisch und pharmazeutisch interessant ist, ist es daher sinnvoll die Zielverbindung enantioselektiv darzustellen. Die Umsetzung von 141 mit (+)-Camphorylsulfonyloxaziridin $^{84}$ war nicht erfolgreich. Nach verlängerter Reaktionsdauer bis zu 7.5 Stunden bei $-78{ }^{\circ} \mathrm{C}$ konnten anhand der ${ }^{1} \mathrm{H}-\mathrm{NMR}$ Analyse des Rohproduktes noch beträchtliche Mengen des Eduktes festgestellt werden. Das gewünschte Produkt war nur in Spuren vorhanden. Eine genauere Reaktionskontrolle mittels Dünnschichtchromatographie war nicht möglich, da Edukt und Produkt in verschiedenen Laufmitteln fast gleiche $\mathrm{R}_{\mathrm{f}}$-Werte aufwiesen.
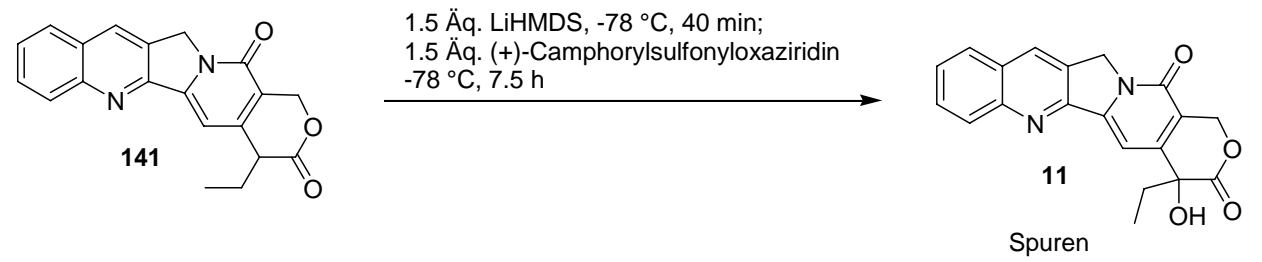

Schema 62. Versuch mit (+)-Camphorylsulfonyloxaziridin

Alternativ war eine enantioselektive Addition von Diethylzink an die Keto-Verbindung 145 in Gegenwart eines Salen-Komplexes als chiralem Induktor vorgesehen. ${ }^{85}$ Die Methode war schon zur enantioselektiven Synthese von (20S)-Camptothecin erfolgreich eingesetzt worden. ${ }^{86}$ Der schnellste Zugang zu 145 bietet eine direkte Dehydrierung von 20 somit anschließende oxidative Spaltung der Doppelbindung. Die Umsetzung von 20 mit DDQ unter Erwärmung auf $100{ }^{\circ} \mathrm{C}$ lieferte jedoch nicht das gewünschte Produkt 144. Neben $40 \%$ des zurückgewonnenen Eduktes wurden nur Zersetzungsprodukte beobachtet. Andere Syntheserouten zur Darstellung von 145 wurden nicht verfolgt, da sie eine deutlich höhere Zahl an Syntheseschritten benötigen. 


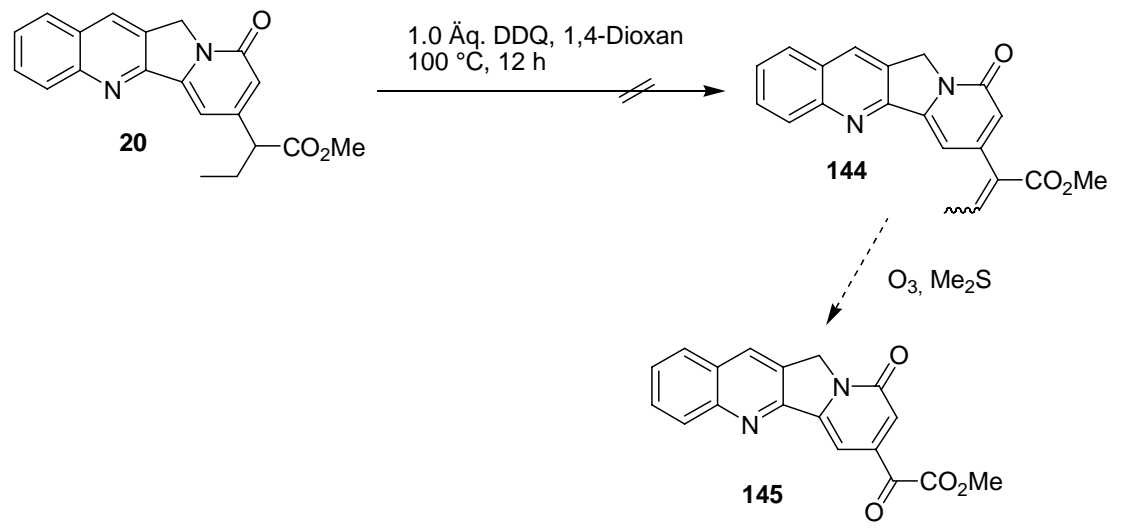

Schema 63. Versuch der Synthese von 144 durch DDQ-Dehydrierung 


\section{Solvolyse-Domino-Reaktion zum Aufbau des Lactonrings}

Da bisherige Versuche einer effizienten Lactonbildung nicht so erfolgreich waren, wurde in der fortlaufenden Arbeit die Modifizierung der Domino-Knoevenagel-hetero-Diels-AlderReaktion und die anschließenden Syntheseschritte untersucht, um die Gesamteffizienz der Totalsynthese von Camptothecin zu erhöhen. Dieser Ansatz erfolgte in Anlehnung an die Untersuchungen von Herrn Bischoff aus dem Arbeitskreis von L. F. Tietze, der den Cbzgeschützten Aldehyd 96 verwendet hatte. Bei der nachfolgenden Domino-Reaktion wurde die Aldehyde 114 und 96 verwendet.

\subsection{Solvolyse-modifizierte Domino-Reaktion und Lactambildung}

Bei einer Solvolyse-modifizierten Domino-Knoevenagel-hetero-Diels-Alder-Reaktion sollte die über Knoevenagel-Kondensation und anschließende hetero-Diels-Alder-Reaktion gebildeten Zwischenprodukte durch nuleophilen Angriff von Alkoholen in das Produkt der Domino-Reaktion umgewandelt werden, wobei eine zusätzliche Estergruppe am Lactonring eingeführt wird (siehe Schema 64 und Mechanismus in Kap. 3.4). Für folgende Versuche wurde Methanol als Nucleophil verwendet werden, da die daraus resultierende Methylestergruppe später leichter zum Alkohol reduziert werden kann. Außerdem sollte Methanol aufgrund der besseren Nucleophilie gegenüber andereren Alkoholen die gewünschte Transformation begünstigen.
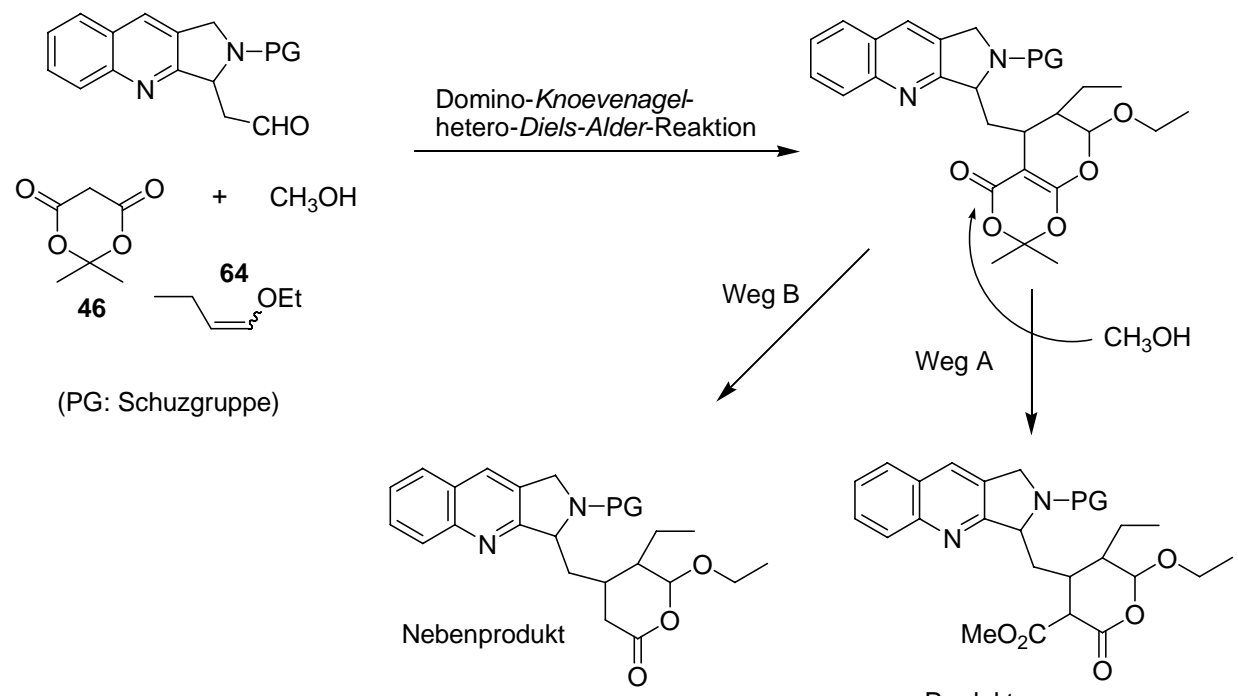

Produkt

Schema 64. Verlauf der methanolyse-modifizierten Domino-Reaktion 


\subsubsection{Optimierung der Methanolyse-Domino-Reaktionen}

Bei der Durchführung einer methanolyse-modifizierten Domino-Knoevenagel-hetero-DielsAlder-Reaktion sollten drei Aspekte berücksichtigt werden:

a) Da Methanol wegen seiner hohen Nucleophilie den Lactonring öffnen kann, sollte die Reaktionstemperatur beachtet werden. $\mathrm{Zu}$ langes Erhitzen oder zu hohe Temperatur sind zu vermeiden.

b) Für die Methanolyse gibt es zwei mögliche Durchführungsarten. Entweder kann das durch Knoevenagel-hetero-Diels-Alder-Reaktion gebildete Cycloaddukt isoliert und dann in Methanol weiter umgesetzt werden. Anderenfalls kann eine Bildung des Cycloadduktes und die anschließende Methanolyse in einer Eintopfreaktion erfolgen.

c) Aufgrund schneller Bildung des normalen Domino-Produktes (als Nebenprodukt an dieser Stelle, siehe Schema 64) bei Raumtemperatur in Abwesenheit von Methanol kann diese Nebenreaktion in Gegenwart von Methanol nicht ausgeschlossen werden. Somit kann das gewünschte Produkt unter den Bedingungen der methanolysemodifizierten Domino-Reaktion verunreinigt werden.

Um eine geeignete Reaktionsbedingung bzw. Temperatur herauszufinden, wurde zuerst eine Testreaktion bei Raumtemperatur mit dem Aldehyd 114 in Gegenwart von aktiviertem Molsieb (3 Å), Dichlormethan und Methanol (1:1) durchgeführt. Unter diesen Bedingungen wurde jedoch nicht die Verbindung 126, sondern ein Gemisch von 125 und 118 durch Massenanalyse beobachtet (siehe Schema 65). Deshalb wurde die Domino-Reaktion in den folgenden Versuchen bei höherer Temperatur $\left(45-55^{\circ} \mathrm{C}\right)$ durchgeführt.

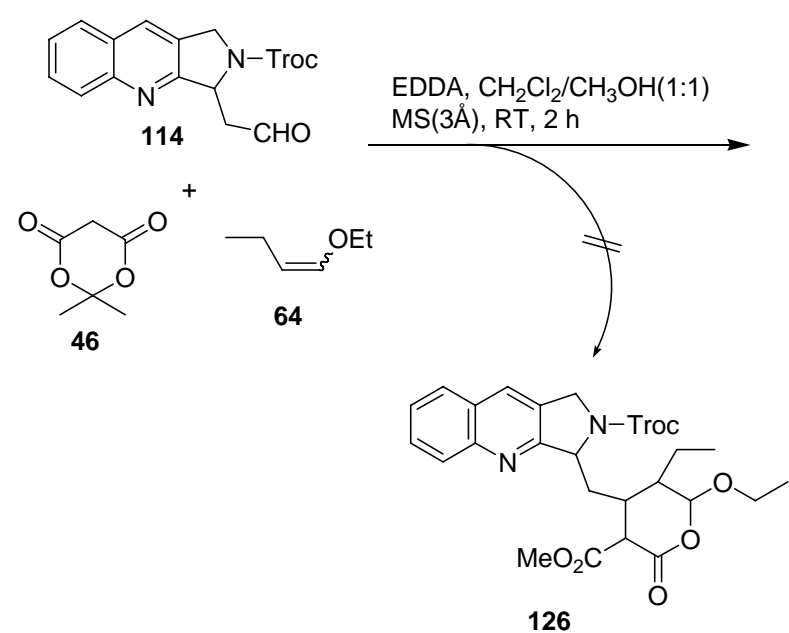

126
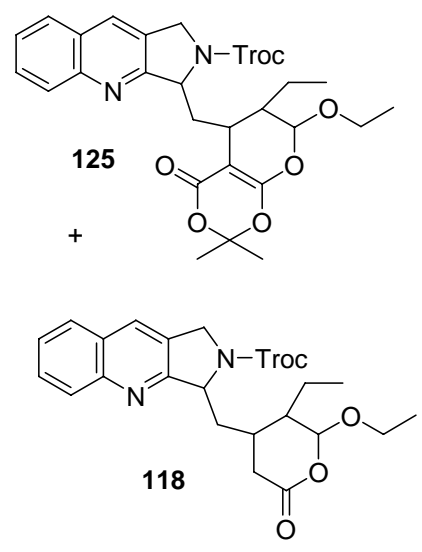

Schema 65. Domino-Reaktion mit Methanol bei Raumtemperatur 
Da Toluol als optimales Lösungsmittel für die ursprüngliche Domino-Reaktion festgestellt wurde, wurde eine zweite Reaktion dieses mal unter Verwendung von 96 in Gegenwart von Methanol (25 Äq.) in Toluol durchgeführt. Nach siebzehn Stunden bei $45{ }^{\circ} \mathrm{C}$ konnte nach chromatographischer Trennung ein Gemisch von drei Verbindungen 146, 121 und 117 in insgesamt 71\% Ausbeute isoliert werden (siehe Schema 66), welche durch hoch aufgelöste Massenspektroskopie bestätigt werden konnten. Dabei wurde als Hauptprodukt 146 gebildet. Das deutet darauf, dass die Domino-Reaktion in Toluol nur langsam verläuft. Dies kann die Nebenproduktbildung von 117 durch thermische Eliminierung von 121 begünstigen. Deshalb wurde für weitere Untersuchungen der Domino-Reaktionen immer Methanol als ausschließliches Lösungsmittel verwendet. Die anschließende Lactambildung durch Umsetzung des Gemisches von 146, 117 und 121 mit $\mathrm{H}_{2}$ in Gegenwart von katalytischen Mengen an $\mathrm{Pd} / \mathrm{C}$ lieferte 62 in $64 \%$ Ausbeute.

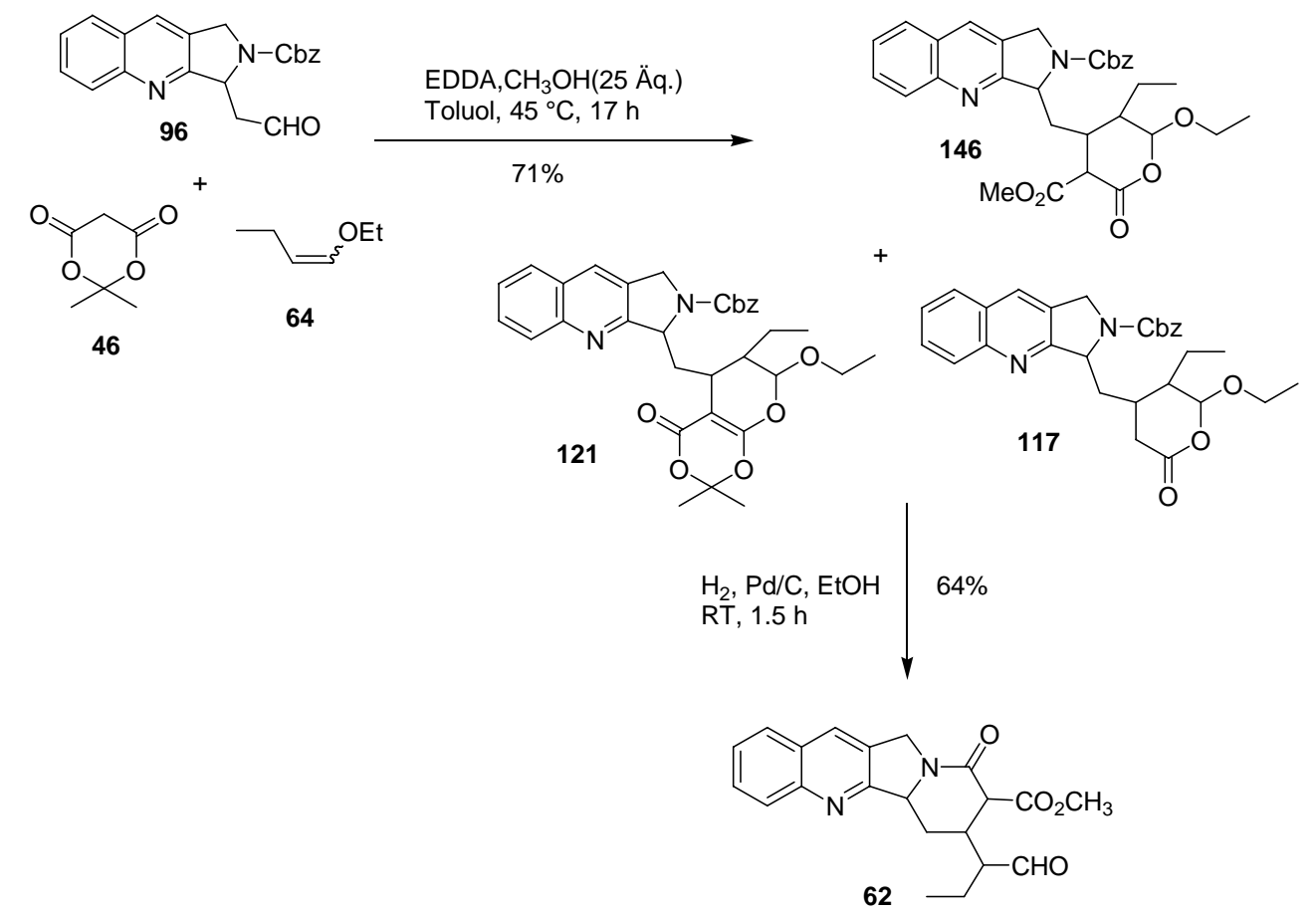

Schema 66. Methanolyse-Domino-Reaktion in Toluol unter Erwärmung

Die dritte Reaktion wurde unter Isolierung des intermediär gebildeten Produktes 121 durchgeführt. 121 wurde durch die übliche Umsetzung mit 96 in Toluol bei Raumtemperatur hergestellt. Nach Konzentration des Lösungsmittels wurde das Rohprodukt 121 in Methanol gelöst und die Reaktionsmischung auf $45^{\circ} \mathrm{C}$ erwärmt. 146 konnte unter diesen Bedingungen in $70 \%$ Ausbeute über zwei Stufen erhalten werden. Allerdings schlug eine anschließende Lactambildung von 62 fehl, was auf Verunreinigungen in 146 zurückzuführen ist. Aus diesem 
Grund sollte die methanolyse-modifizierte Domino-Reaktion nicht in separaten Stufen durchgeführt werden.

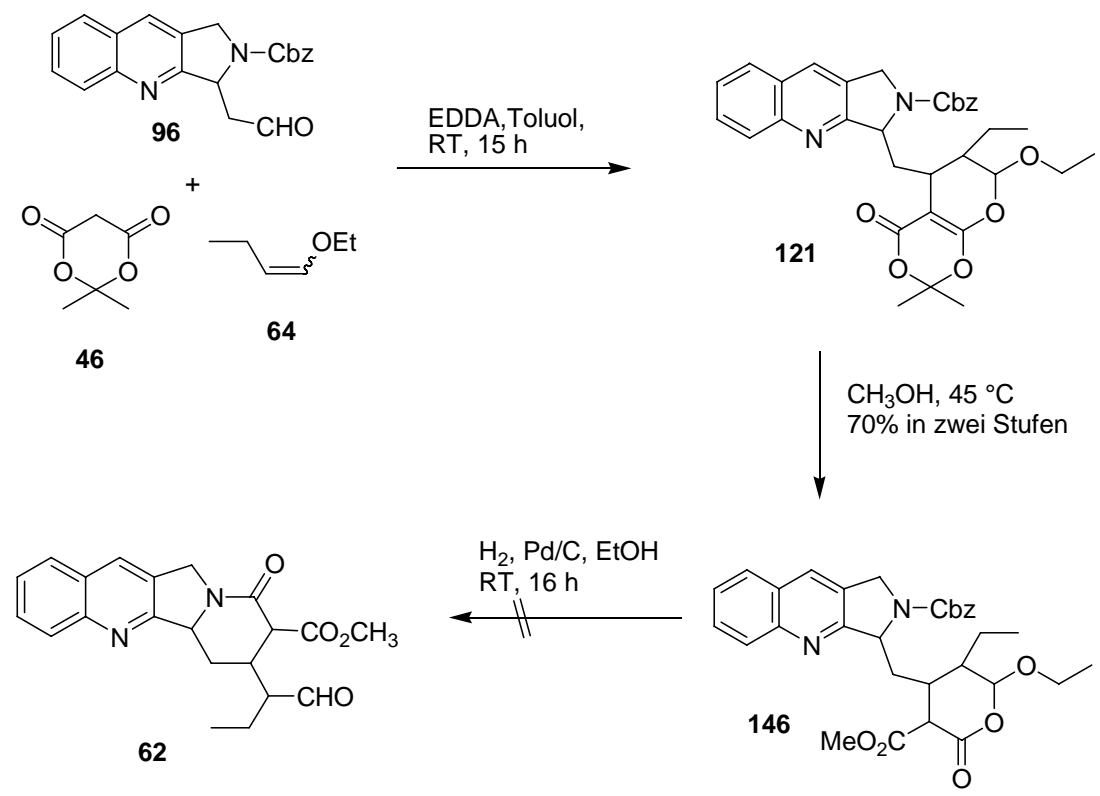

Schema 67. Methanolyse-Domino-Reaktion mit Isolierung des Zwischenproduktes 121

\subsubsection{Durchführung der Methanolyse-Domino-Reaktion}

Basierend auf obigen Ergebnissen wurden zur methanolyse-modifizierten Domino-Reaktion alle Reagenzien in Methanol in einem Druckkolben umgesetzt. Ausgehend von dem N-Trocgeschützten und N-Cbz-geschützten Aldehyden 114 bzw. 96 konnten Domino-Produkte 126 und 146 jeweils in 85\% und 76\% Ausbeute dargestellt werden (siehe Tabelle 14). Die Zugabe von $3 \AA$ Molsieb oder Methylorthoacetat sollte die möglicherweise durch $\mathrm{H}_{2} \mathrm{O}$ ausgelöste Nebenreaktion unterdrücken, es konnte aber keine signifikante Verbesserung der Ausbeute erreicht werden (Eintrag 3 und 4).

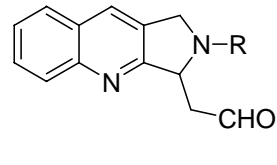

114: $R=$ Troc 96: $\mathrm{R}=\mathrm{Cbz}$

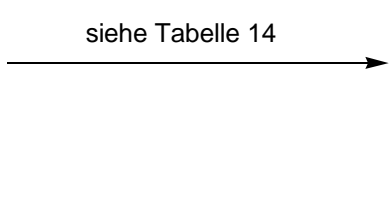

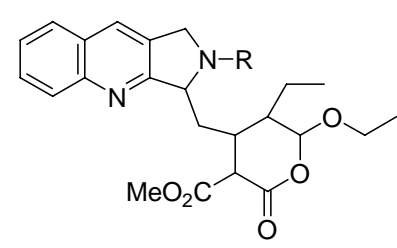

126: $R=$ Troc 


\begin{tabular}{|c|c|c|}
\hline Eintrag & Reaktionsbedingungen & Ausbeute \\
\hline 1 & $\begin{array}{l}\text { 1.0 Äq. 114, } 4.0 \text { Äq. Enolether 64, } 1.1 \text { Äq. Meldrum-Säure, aktiv. } \\
\text { Molsieb (3 A, Pulver), EDDA in Methanol, } 50^{\circ} \mathrm{C}, 2.5 \mathrm{~h}\end{array}$ & $80 \%$ \\
\hline 2 & $\begin{array}{l}\text { 1.0 Äq. 114, 4.0 Äq. Enolether 64, } 1.1 \text { Äq. Meldrum-Säure, } 2.0 \text { Äq. } \\
\text { Methylorthoacetat, EDDA in Methanol, } 45^{\circ} \mathrm{C}, 2 \mathrm{~h}\end{array}$ & $85 \%$ \\
\hline 3 & $\begin{array}{l}1.0 \text { Äq. 96, } 4.0 \text { Äq. Enolether 64, } 1.1 \text { Äq. Meldrum-Säure, } 2.0 \text { Äq. } \\
\text { Methylorthoacetat, aktiv. Molsieb ( } 3 \text { A, Kugel), EDDA in Methanol, } \\
45^{\circ} \mathrm{C}, 2 \mathrm{~h} 45 \mathrm{~min}\end{array}$ & $73 \%$ \\
\hline 4 & $\begin{array}{l}1.0 \text { Äq. 96, } 3.0 \text { Äq. Enolether 64, } 1.0 \text { Äq. Meldrum-Säure, EDDA in } \\
\text { Methanol, } 55^{\circ} \mathrm{C}, 3 \mathrm{~h}\end{array}$ & $76 \%$ \\
\hline
\end{tabular}

Tabelle 14. Methanolyse-Domino-Reaktion von 114 und 96

\subsubsection{Lactambildung}

Ausgehend von 146 erfolgte die Cbz-Entschützung und intramolekulare Lactambildung zu 62 in $72 \%$ Ausbeute. Die Bildung von 62 gelang auch durch Umsetzung von 126 mit Zn und HOAc in THF, wobei die Reaktion durch Wasserzugabe (Volumen THF/ $\mathrm{H}_{2} \mathrm{O}=80: 1$ ) dramatisch beschleunigt. Unter Standard-Bedingungen (aktiviertes Zink-Pulver/Essigsäure) ließ sich keine gewünschte Reaktion beobachten. Erst nach Einsatz von Wasser als Additiv konnte ein Umsatz beobachtet werden.
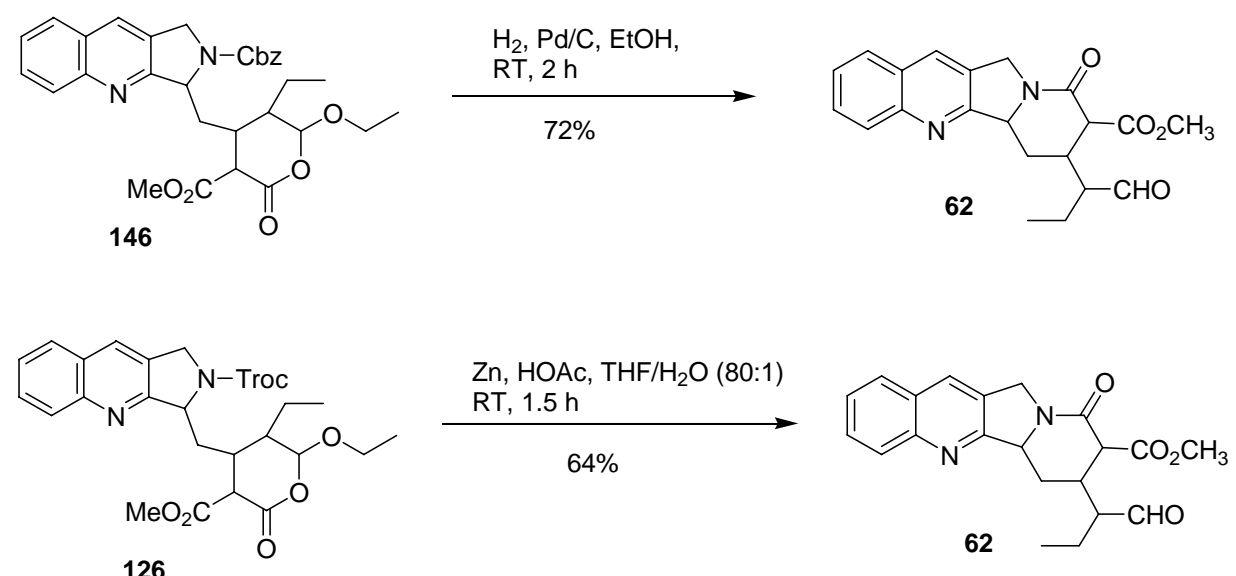

Schema 69. Lactambildung aus 146 und 126 


\subsection{Aufbau des Lactonrings}

Um den Lactonring aufzubauen muss die Methylestergruppe zum Alkohol reduziert werden. Dabei ist es erforderlich, die Aldehydgruppe von 62 vorher in eine funktionale Gruppe zu überführen, die unter den Methylester-Reduktionsbedingungen inert ist. Damit ergeben sich vier Möglichkeiten: Carboxyl, Acetale, Methylester oder tert-Butylester.

\subsubsection{Reduktive Differenzierung zwischen Methylester- und Carboxylgruppe}

Der Aldehyd 62 wurde nach einer Vorschrift von Herrn Bischoff zuerst durch Oxidation mit $\mathrm{NaClO}_{2}$ in die Carbonsäure 147 überführt. Die Differenzierung der Methylester- und Carboxylgruppe wurde durch Reduktion mit $\mathrm{NaBH}(\mathrm{OMe})_{3}$ in 1,2-Dimethoxyethan (DME) ${ }^{87}$ versucht (siehe Tabelle 15). Da die DC-Kontrolle keinen Hinweis auf die Bildung von Produkten geringerer Polarität gab und man annehmen konnte, dass der Lactonring unter den Reaktionsbedingungen nicht geschlossen sein konnte, wurde das Rohprodukt direkt mit TMS$\mathrm{CHN}_{2}$ umgesetzt, um die Carboxylgruppe in den Methylester zu überführen und so die Lactonisierung zu erleichtern. ${ }^{88}$ Durch hoch aufgelöste Massenspektroskopie ließen sich die drei Produkte 148, 149 und 131 nachweisen, wobei 148 das Hauptprodukt darstellte. Da weder das Edukt noch das Reduktionsmittel gut löslich in DME waren, wurde THF als Lösungsmittel für die obige Umsetzung verwendet. Trotz der guten Löslichkeit wurde keine deutliche Verbesserung beobachtet. Bei der Untersuchung mit DIBAL-H bei $-78{ }^{\circ} \mathrm{C}$ wurde neben dem Edukt das gewünschte Produkt 131 in Spuren beobachtet. Die Umsetzung führte außerdem zu einem Massenverlust von 50\%.

\begin{tabular}{|c|c|c|}
\hline Eintrag & Reaktionsbedingungen & Ergebnisse \\
\hline 1 & $\begin{array}{l}1.0 \text { Äq. 147, } 4.0 \text { Äq. } \mathrm{NaBH}(\mathrm{OMe})_{3}, \mathrm{DME}, 85^{\circ} \mathrm{C} \text {, } \\
80 \text { min; dann } 1.0 \text { Äq. TMS-CHN } 2 \text {, Methanol/ } \\
\text { Toluol, RT, } 80 \text { min }\end{array}$ & $\begin{array}{c}148 \\
\text { (Spuren) } 149,131\end{array}$ \\
\hline 2 & $\begin{array}{l}1.0 \text { Äq. 147, } 4.0 \text { Äq. } \mathrm{NaBH}(\mathrm{OMe})_{3}, \mathrm{THF}, 65^{\circ} \mathrm{C}, 4 \\
\text { h; dann } 1.2 \text { Äq. TMS-CHN } 2 \text {, Methanol/Toluol, RT, } \\
80 \text { min }\end{array}$ & $\begin{array}{c}148 \\
\text { (Spuren) } 131\end{array}$ \\
\hline 3 & 1.0 Äq. 147, 3.2 Äq. DIBAL-H, $-78^{\circ} \mathrm{C}, 1 \mathrm{~h}$ & (Spuren) 131 \\
\hline
\end{tabular}



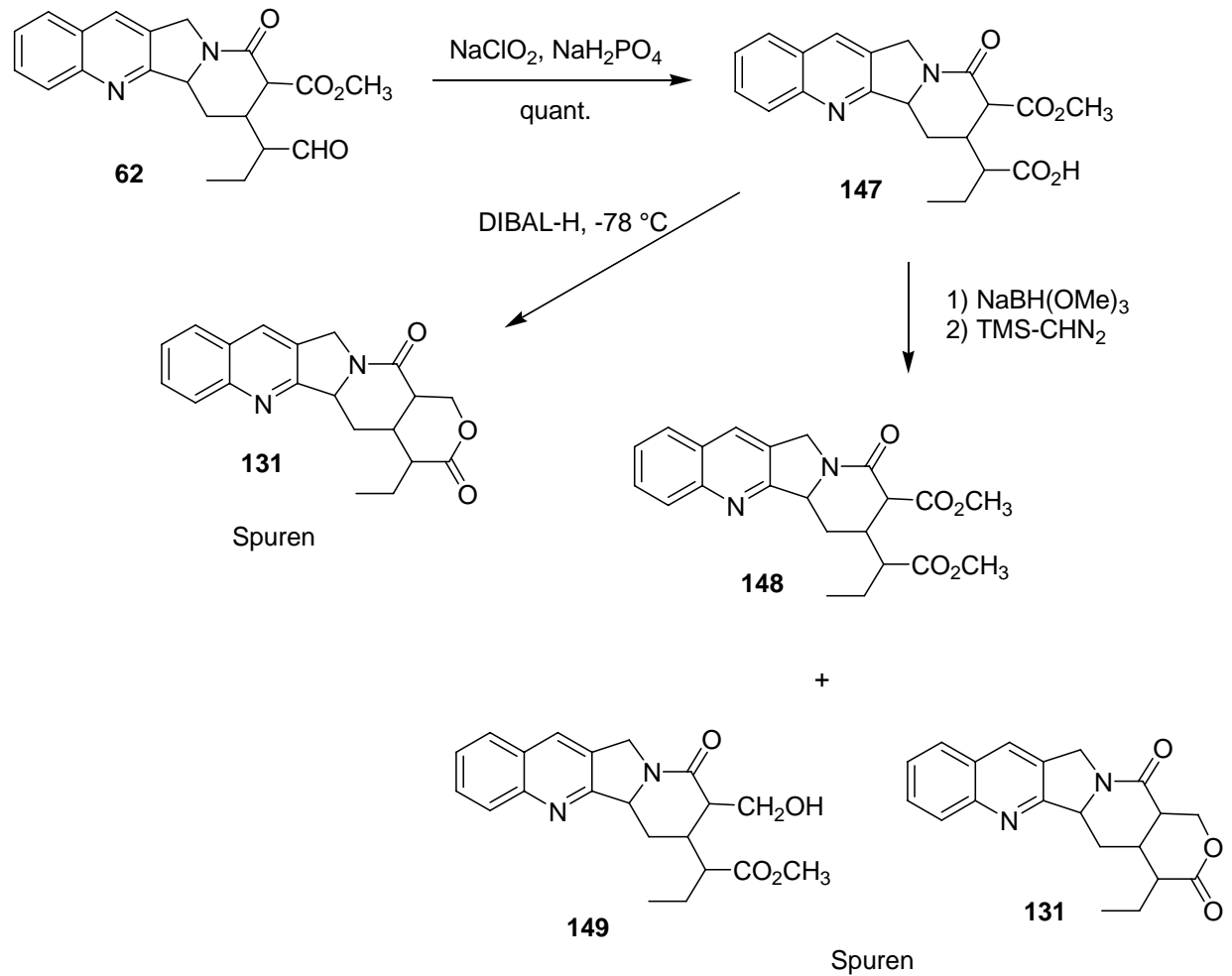

Schema 70. Untersuchung zur selektiven Reduktion der Carbonsäure 147

Da Carboxylate generell inert gegenüber den Reduktionsbedingungen sind, ${ }^{66}$ wurde das Lithiumsalz der Carbonsäure 147 zur weiteren Untersuchung durch Umsatz mit LiOEt dargestellt. Während keine Verbesserung bei Umsatz mit $\mathrm{NaBH}(\mathrm{OMe})_{3}$ beobachtet werden konnte, führte die Verwendung von $\mathrm{LiBH}_{4}$ zur Zersetzung, wobei über 90\% Massenverlust beobachtet wurde.
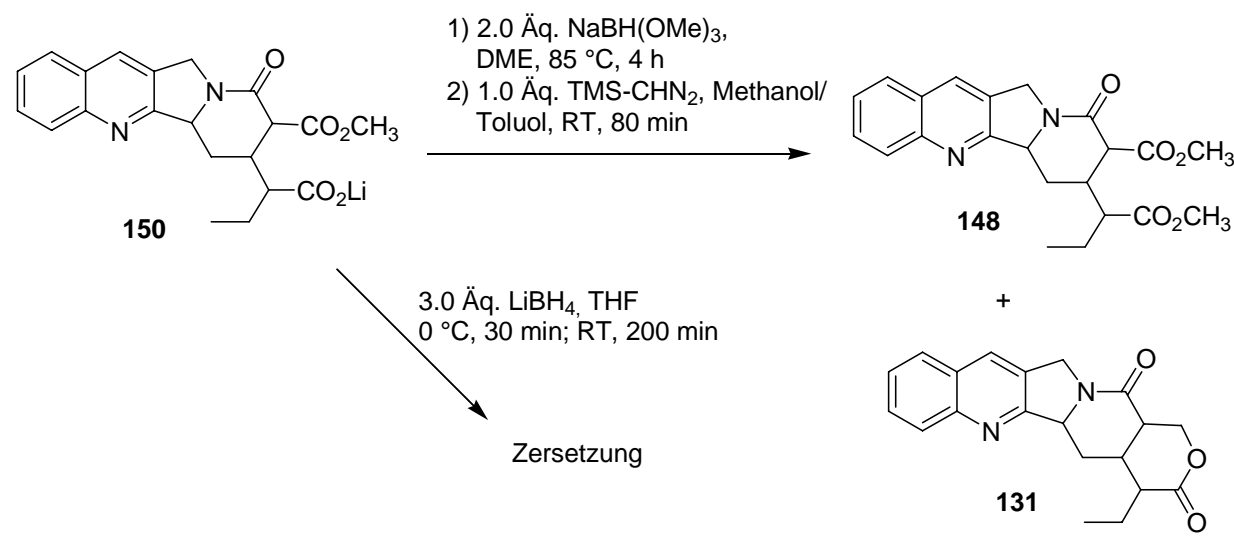

Spuren

Schema 71. Untersuchung der selektiven Reduktion des Salzes von 147 


\subsubsection{Acetal-Schützung des Aldehyds 62}

Um die Aldehydgruppe von 62 zu schützen und nachher wieder effektiv zu entschützen, sollte das Acetal 151 hergestellt werden, da acyclische Acetale im Vergleich zu cyclischen Acetale generell leichter hydrolysiert werden können. Die $\mathrm{RuCl}_{3}$-katalytisierte Acetalisierung und anschließende DDQ-vermittelte Dehydrierung lieferten 152 in 43\% Ausbeute über zwei Stufen. Die Reduktion mit DIBAL-H im Überschuss bei $-78^{\circ} \mathrm{C}$ lieferte ein Gemisch von 153 und 154. Die direkte Umsetzung des Gemisches mit $\mathrm{NaBH}_{4}$ in Methanol gab den Alkohol 154 in $71 \%$ Ausbeute, wobei 6\% des Eduktes 152 zurückgewonnen werden konnten.
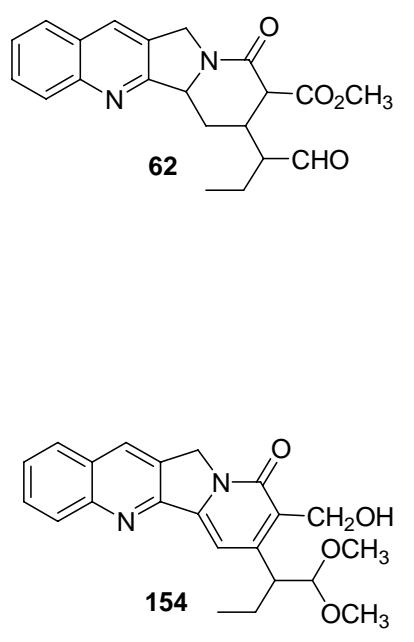

1.2 Äq. $\mathrm{HC}\left(\mathrm{OCH}_{3}\right)_{3}$, kat. $\mathrm{RuCl}_{3} \cdot \mathrm{H}_{2} \mathrm{O}, 60^{\circ} \mathrm{C}, 3.5 \mathrm{~h}$ $54 \%$ $\rightarrow$ 1) 2.0 Äq. DIBAL-H, $\mathrm{CH}_{2} \mathrm{Cl}_{2}$ $-78^{\circ} \mathrm{C}, 1.5 \mathrm{~h}$

2) $\mathrm{NaBH}_{4}, \mathrm{CH}_{3} \mathrm{OH},-78{ }^{\circ} \mathrm{C}, 1 \mathrm{~h}$, dann RT, 40 min

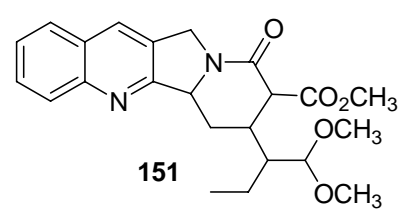

\begin{tabular}{l|l}
$79 \%$ & $\begin{array}{l}2.2 \mathrm{Äq} . \mathrm{DDQ}, 1,4-D i o x a n \\
65^{\circ} \mathrm{C}, 1 \mathrm{~h}\end{array}$
\end{tabular}

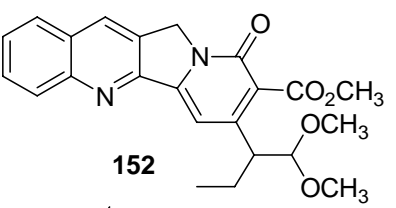

DIBAL-H, $-78^{\circ} \mathrm{C}$

153
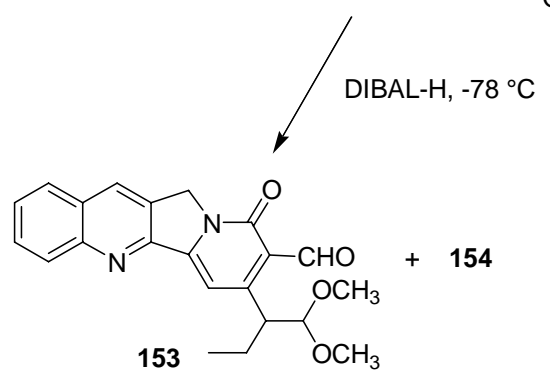

Schema 72. Synthese von 154

Die nachfolgende Entschützung der Acetalgruppe erwies sich jedoch als problematisch. Während wässrige TFA bei Raumtemperatur keine Umsetzung ermöglichte, führte $p$-TSA beim Erwärmen zur Zersetzung.
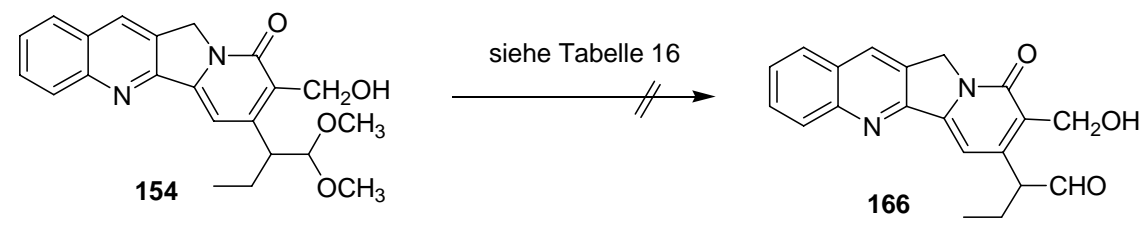


\begin{tabular}{|c|c|c|}
\hline Eintrag & Reaktionsbedingungen & Ergebnisse \\
\hline 1 & $\begin{array}{l}1.0 \text { Äq. 154, } 1.9 \text { Äq. TFA (aq. } 0.2 \mathrm{M} \text { ) in } \mathrm{CH}_{2} \mathrm{Cl}_{2}, \mathrm{RT} \text {, } \\
35 \text { min; dann } 3.8 \text { Äq. TFA (aq. } 0.2 \mathrm{M} \text { ), RT, } 2.5 \mathrm{~h}\end{array}$ & Edukt \\
\hline 2 & $\begin{array}{l}1.0 \text { Äq. 154, } 3.8 \text { Äq. TFA (aq. } 0.2 \mathrm{M} \text { ) in THF, } 45^{\circ} \mathrm{C} \text {, } \\
70 \text { min }\end{array}$ & Edukt \\
\hline 3 & $\begin{array}{l}1.0 \text { Äq. 154, } 3.0 \text { Äq. p-TSA. } \mathrm{H}_{2} \mathrm{O} \text {, in Aceton/Wasser } \\
(2: 1), 55^{\circ} \mathrm{C}, 2 \mathrm{~h}\end{array}$ & Zersetzung \\
\hline
\end{tabular}

Tabelle 16. Untersuchung der Entschützung des Acetals 154

Unter Verwendung von $\mathrm{LiBF}_{4}{ }^{89}$ in einem Acetonitril/Wasser Gemisch konnten der Enolether 155 und das Lactol 156 in 60\% bzw. 40\% Ausbeute isoliert werden. Interessanterweise lässt sich der Enolether 155 durch modifizierter Sharpless-Dihydroxylierung zur enantionselektiven Synthese von Camptothecin nutzen. ${ }^{90}$ Die Überführung des Lactols 156 in das Lacton 141 mit PCC war bereits literaturbekannt. ${ }^{91}$

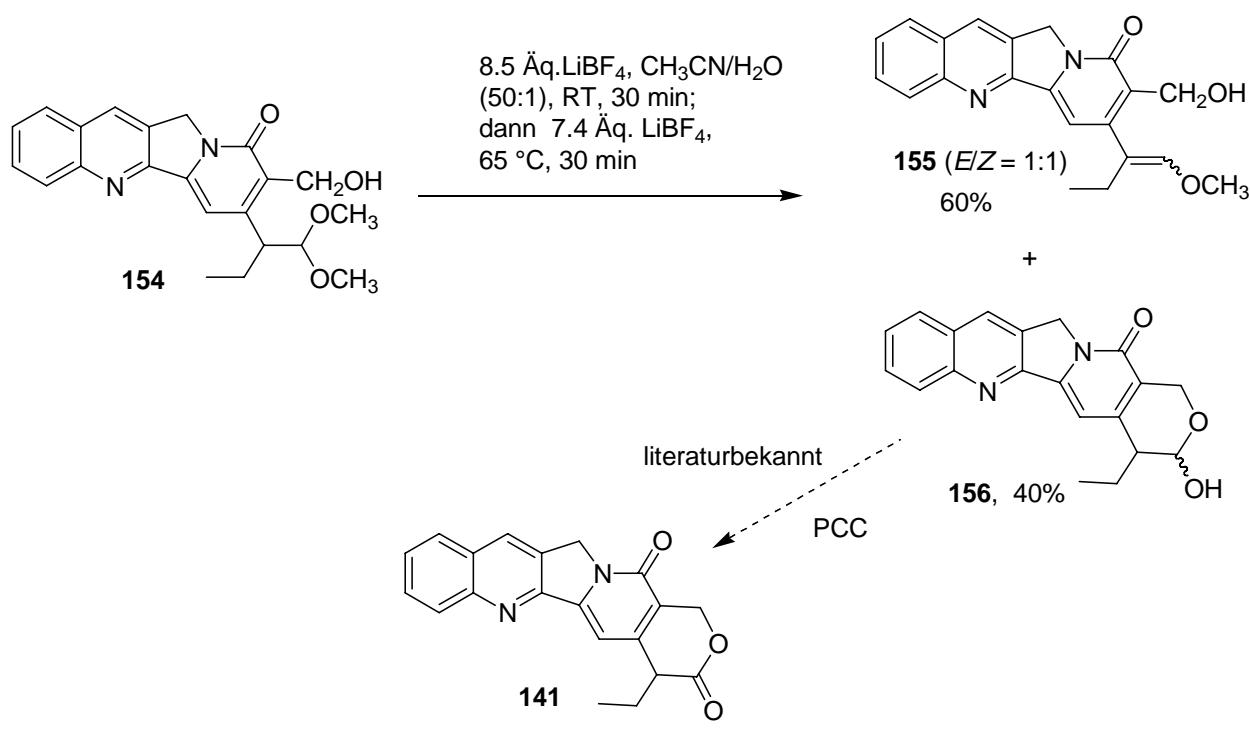

Schema 74. Entschützung des Acetals 154

Obwohl die endgültige Zielverbindung über die Acetalbildung des Aldehyds 62 erreicht werden konnte, wies die Gesamtsynthese nur eine niedrige Gesamtausbeute auf. Es wurde daher noch ein weiterer Syntheseweg untersucht. 


\subsubsection{Regioselektive Reduktion des Methylesters 157}

\subsubsection{Synthese des Methylesters 157}

Der neue Ansatz zur Synthese von Camptothecin sah die Synthese von 157 als Zwischenstufe vor. Die Veresterung der Cabonsäure 147 mit Trimethylsilyldiazomethan lieferte 148 in 76\% Ausbeute. Die anschließende Dehydrierung mit DDQ lieferte den Methylester 157 mit einer Pyridon-Komponente in 51\% Ausbeute.

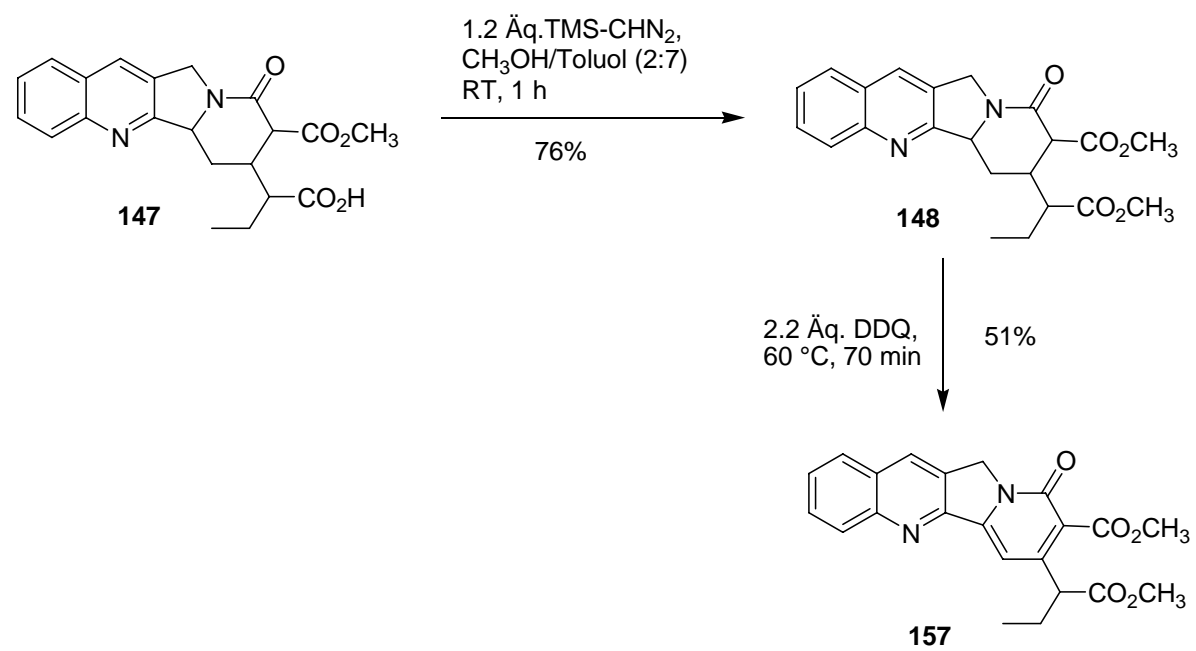

Schema 75. Synthese von 157

\subsubsection{Reduktion mit DIBAL-H}

Eine regioselektive Reduktion von 157 wurde zuerst unter Verwendung von DIBAL-H untersucht. Der Methylester 157 wurde bei $-78{ }^{\circ} \mathrm{C}$ mit einer verdünnten Lösung von DIBAL-H in stöchiometrischen Mengen umgesetzt. Es wurde nur das Edukt quantitativ zurückgewonnen werden. Es war $\mathrm{zu}$ beachten, dass direkte Zugabe von kommerziell erhältlicher DIBAL-H Lösung (1 M in Dichlormethan) zur partiellen Zersetzung des Substrates führte.

Beim zweiten Versuch wurde DIBAL-H dreimal in bestimmten Zeitabständen in insgesamt 3.35 Äquivalenten zugegeben (siehe Schema 76), um das Edukt 157 vollständig umzusetzten. Es wurde der Aldehyd 158 in 5\% und das Lactol 156 in weniger als 1\% Ausbeute isoliert. Zusätzlich entstand in größerer Menge ein Gemisch von höherer Polarität, dessen Strukturaufklärung jedoch nicht gelang. Aufgrund massenspektrometrischer Untersuchungen konnte aber eine Bildung des Diols 159 ausgeschlossen werden. 

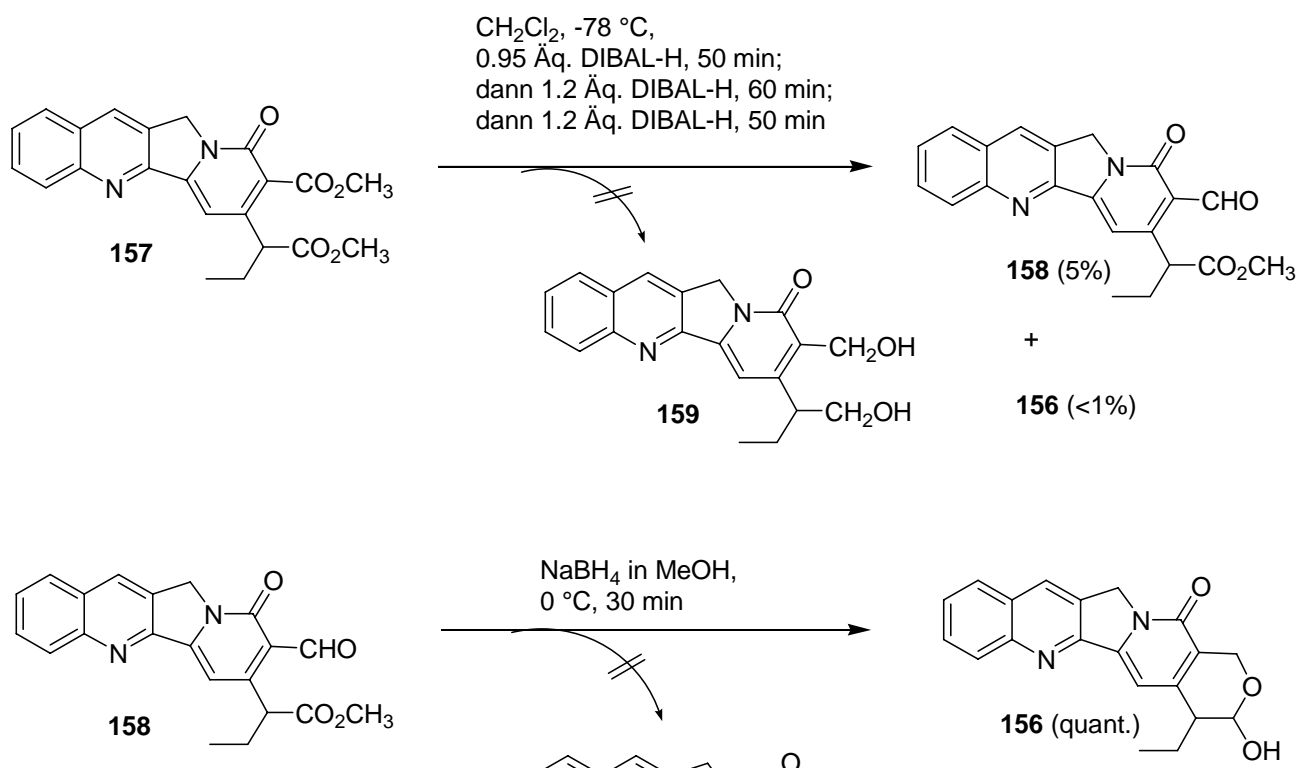

Schema 76. Regioselektive Reduktion von 157 und Synthese des Lactols 156

Da das Signal für das Aldehyd-Proton von 158 in Form eines Singuletts beobachtet wurde, muss sich die Formylgruppe am Pyridon-Ring befinden. Diese Vermutung wurde durch die Reaktion des Aldehyds 158 mit $\mathrm{NaBH}_{4}$ zum Lactol 156, einer literaturbekannten Verbindung, ${ }^{91}$ bestätigt. Das gewünschte Produkt 141 wurde unter den angegebenen Bedingunen jedoch nicht gebildet.

Bei dem weiteren Versuch wurde die Reaktionmischung nach Zugabe von DIBAL-H von $-78{ }^{\circ} \mathrm{C}$ auf Raumtemperatur erwärmt. Neben $31 \%$ des zurückgewonnenen Eduktes konnte der iso-Butylester 160 in 66\% Ausbeute isoliert werden (siehe Schema 77). Durch Vergleich des NMR-Spektrums von 158 oder 157 mit 160 konnte die Position des iso-Butylesters zugeordnet werden.
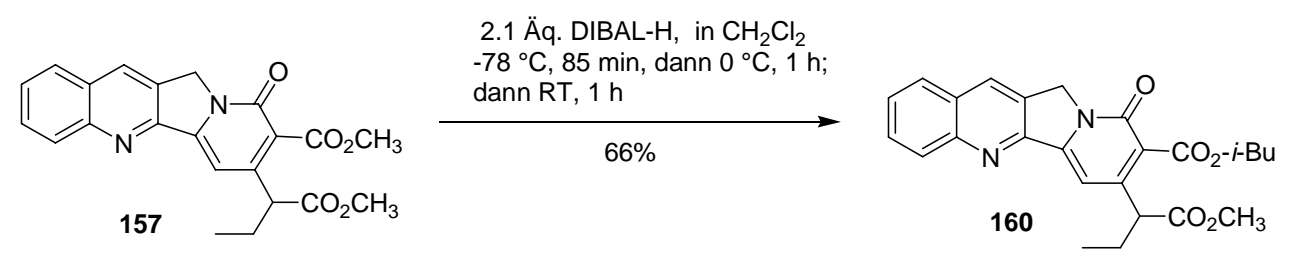
Obwohl es nicht gelang, 141 selektiv darzustellen, lieferten die Untersuchungen wichtige Informationen über die Reaktivität der Estergruppen. Dabei stellte sich heraus, dass die direkt am Pyridonring gebundene Esterfunktionalität eine höhere Reaktivität aufweist.

\subsubsection{3}

\section{Diskussion ausgewählter spektroskopischer Daten der Verbindungen 158,} 157 und 160

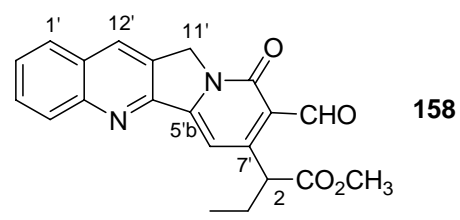

Das ${ }^{1}$ H-NMR-Spektrum (siehe Abbildung 11) der Verbindung 158 zeigt im Tieffeldbereich bei $\delta=7.70-8.44$ die Signale mit dem typischen Kopplungsmuster der Chinolin-Einheit. Das Singulett bei $\delta=8.44$ lässt sich dem Proton an C-12' zuordnen. Die Protonen an C-1' und C4' resonieren als Dublett jeweils bei $\delta=7.97$ mit einer ortho-Kopplungskonstante von $J=8.1$ $\mathrm{Hz}$ und 8.26 mit einer ortho-Kopplungskonstante von $J=8.7 \mathrm{~Hz}$. Für 2'-H und 3'-H findet man die Signale als zentrietes Multiplett bei $\delta=7.70$ bzw. 7.86. Das Singulett bei $\delta=10.67$ ist charakteristisch für das Proton der Aldehydgruppe. Das Proton an C-6' resoniert als Singulett bei $\delta=7.41$. Da die zwei diastereotopen Protonen an C-11' weiter vom stereogenen Zentrum entfernt sind, lässt sich denen das Singulett bei $\delta=5.34$ zuordnen. Das Proton an C-2 resoniert als Triplett $\left({ }^{3} J=7.5 \mathrm{~Hz}\right)$ bei $\delta=5.22$. Im Hochfeldbereich werden das Singulett bei $\delta=3.71$ für die Methylestergruppe und das Triplett $\left({ }^{3} J=7.6 \mathrm{~Hz}\right)$ bei $\delta=0.99$ für die Methylgruppe identifiziert. Den zwei diastereotopen Protonen an C-3 sind die Signale als Multiplett bei $\delta=2.17-2.34$ bzw. 1.80-1.98 zuzuordnen.

Das hoch aufgelöste ESI-Massenspektrum weist ein Signal bei $m / z=363.1341$ für $[\mathrm{M}+\mathrm{H}]^{+}$ (Ber. $m / z=363.1339$ ) auf. 


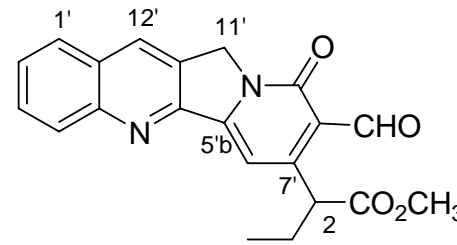

158

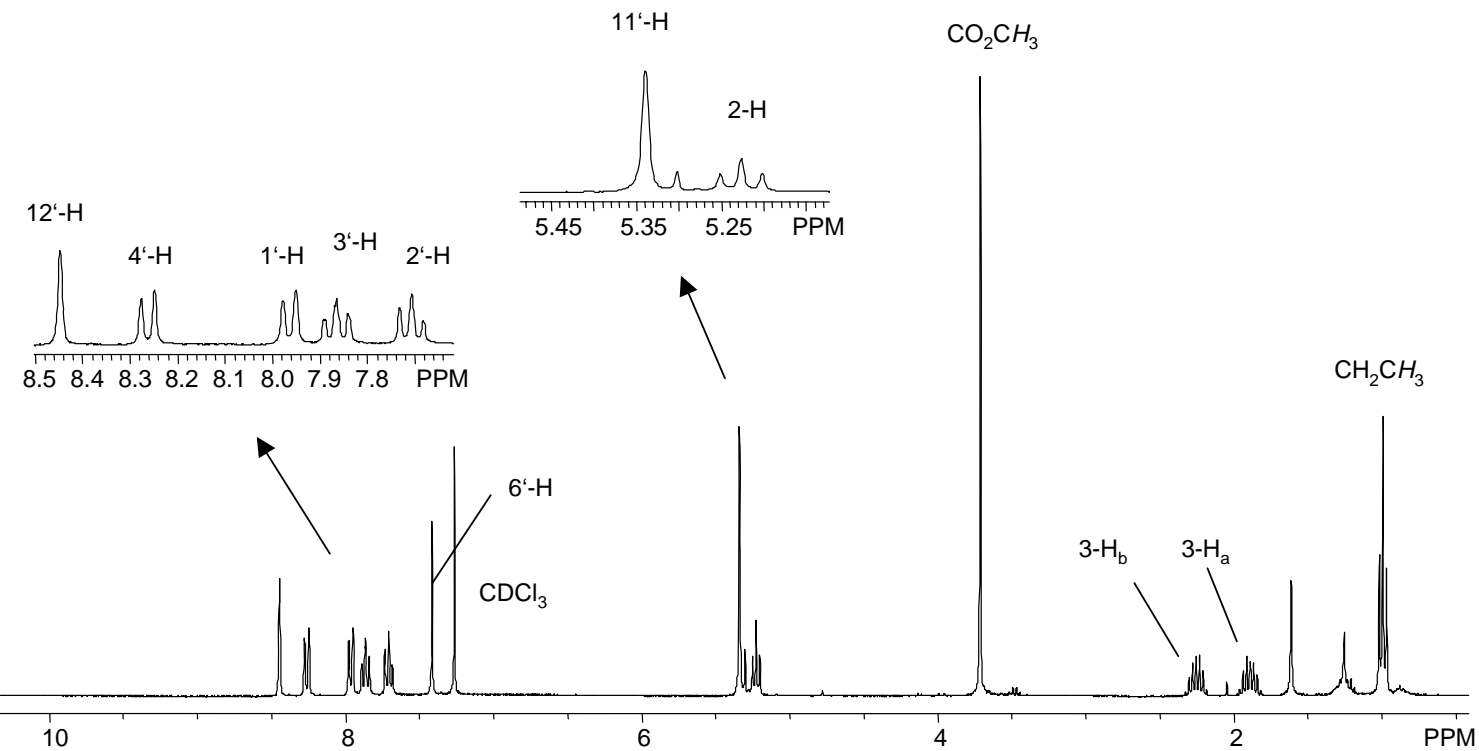

Abbildung 11. ${ }^{1} \mathrm{H}-\mathrm{NMR}\left(\mathrm{CDCl}_{3}, 300 \mathrm{M}\right)$ von 158 


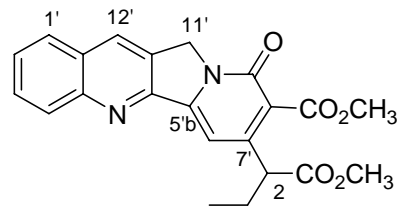

157

Das ${ }^{1}$ H-NMR-Spektrum (siehe Abbildung 12) der Verbindung 157 stimmt sehr gut mit der Literatur $^{92}$ überein. Es ist bemerkungswert, dass sich die zwei Estergruppen von 157 durch Vergleich des ${ }^{1}$ H-NMR-Spektrums mit 158 identifizieren lassen, bzw. das Singulett bei $\delta=$ 3.99 der direkt am Pyridonring gebundenen Estergruppe und das andere Singulett bei $\delta=3.72$ der unteren Estergruppe zuzuordnen ist. Das IR-Spektrum weist drei Banden bei $\widetilde{v}=1734$, 1653 und $1606 \mathrm{~cm}^{-1}$ für die $\mathrm{C}=\mathrm{O}$ Streckschwingungen sowie zwei Banden bei $\widetilde{v}=1169$ und $1128 \mathrm{~cm}^{-1}$ für die C-O Streckschwingungen auf. Das hoch aufgelöste ESI-Massenspektrum weist ein Signal bei $m / z=393.1444$ für $[\mathrm{M}+\mathrm{H}]^{+}($Ber. $m / z=393.1445)$ auf.

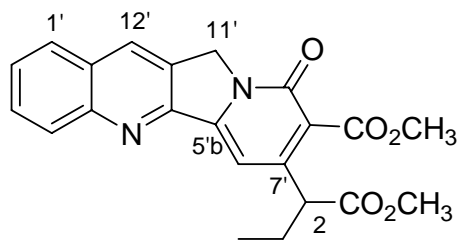

157
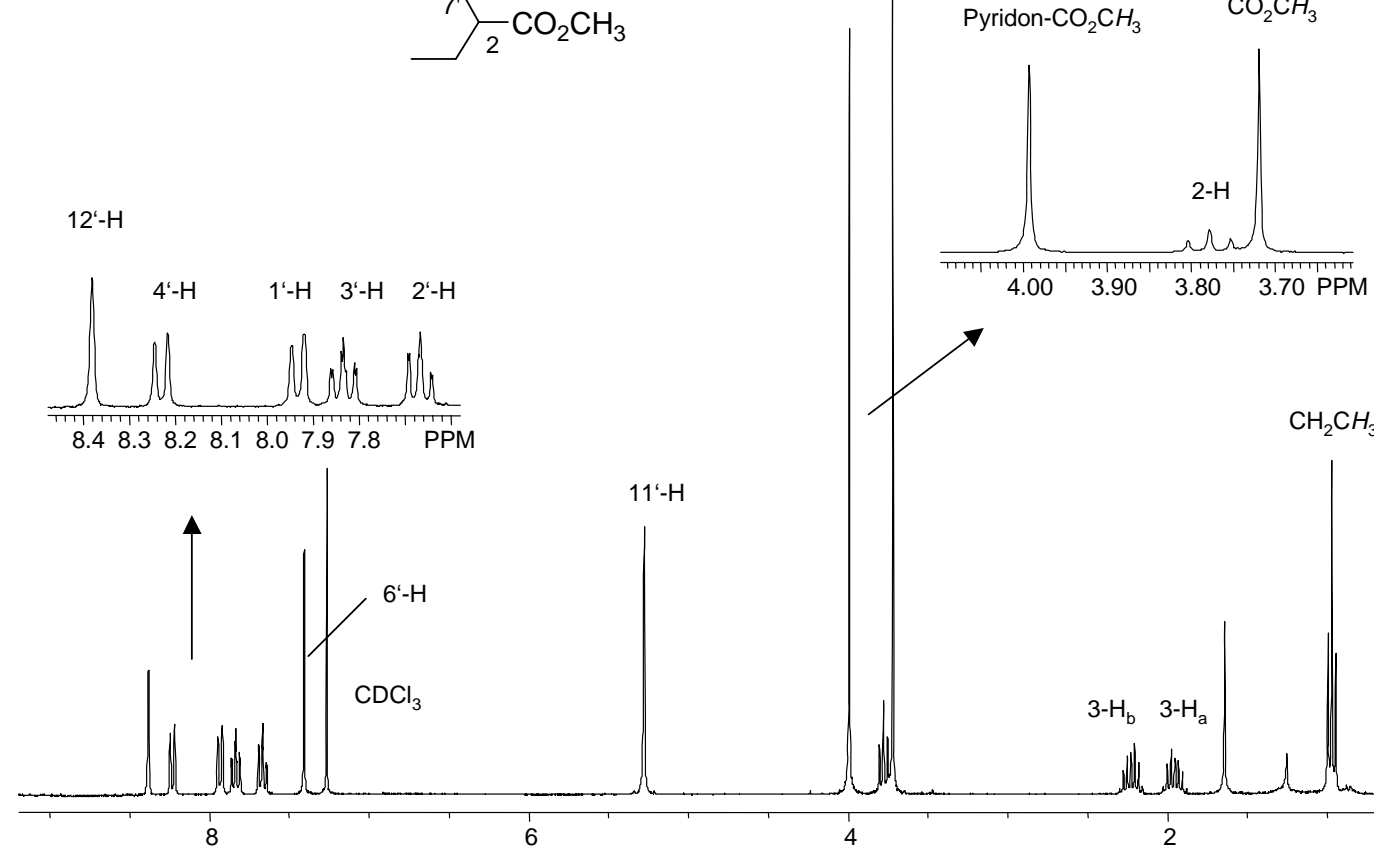

Abbildung 12. ${ }^{1} \mathrm{H}-\mathrm{NMR}\left(\mathrm{CDCl}_{3}, 300 \mathrm{M}\right)$ von 157 


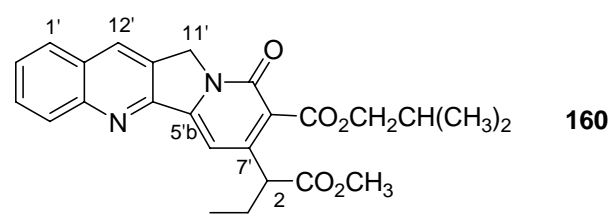

Das ${ }^{1}$ H-NMR-Spektrum (siehe Abbildung 13) der Verbindung 160 ist denen von 158 bzw. 157 gut vergleichbar. Neben dem typischen Kopplungsmuster der Chinolin-Einheit im Tieffeldbereich bei $\delta=7.66-8.37$ für die Protonen an C-12', C-4', C-1', C-3' und C-2' werden das Singulett bei $\delta=7.40$ für das Proton an C-6' und das andere Singulett bei $\delta=5.26$ für die zwei diastereotopen Protonen an C-11' identifiziert. Die Methylestergruppe und 2-H resonieren als Multiplett bei $\delta=3.63-3.84$ zusammen. Im Hochfeldbereich findet man das Triplett bei $\delta=0.97$ mit einer Kopplungskonstante von $J=7.2 \mathrm{~Hz}$ für 4-H und das Dublett bei $\delta=1.04$ mit einer Kopplungskonstante von $J=6.8 \mathrm{~Hz}$ für die zwei Methylgruppen des iso-Butylesters. Der Methylengruppe des iso-Butylesters lässt sich das Signal als zentriertes Multiplett bei $\delta=4.21$ zuordnen. Über ein ${ }^{1} \mathrm{H}-{ }^{1} \mathrm{H}-$ Korrelationsexperiment wurde ein diastereotopes Proton an C-3 als Multiplett bei $\delta=1.87-2.03$ identifiziert. Das andere diastereotope Proton an C-3 und das restliche Proton des iso-Butylesters treten als Multiplett bei $\delta=2.03-2.32$ zusammen auf.

Entsprechend weist das IR-Spektrum drei Banden bei $\widetilde{v}=1737,1657$ und $1611 \mathrm{~cm}^{-1}$ für die $\mathrm{C}=\mathrm{O}$ Streckschwingungen und zwei Banden bei $\widetilde{v}=1170$ und $1126 \mathrm{~cm}^{-1}$ für die $\mathrm{C}-\mathrm{O}$ Streckschwingungen auf. Das hoch aufgelöste ESI-Massenspektrum weist ein Signal bei $m / z$ $=435.1915$ für $[\mathrm{M}+\mathrm{H}]^{+}($Ber. $m / z=435.1914)$ auf. 


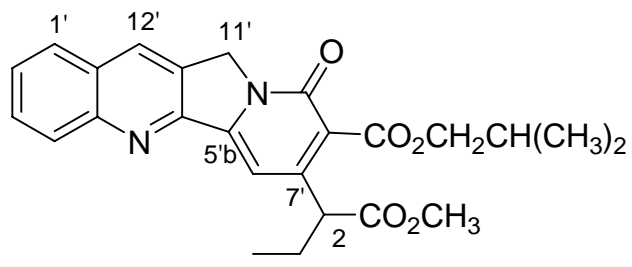

160
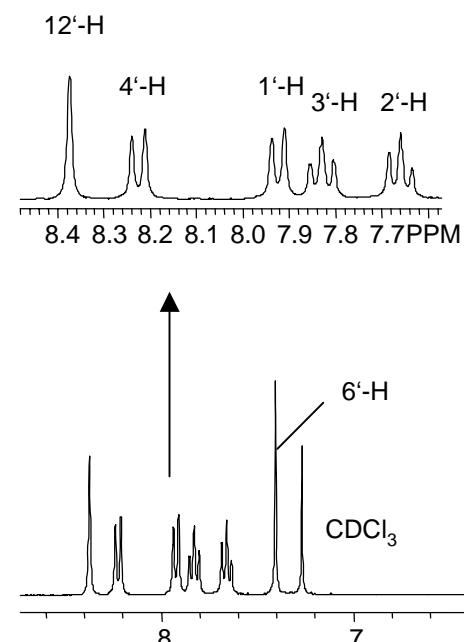

Abbildung 13. ${ }^{1} \mathrm{H}-\mathrm{NMR}\left(\mathrm{CDCl}_{3}, 300 \mathrm{M}\right)$ von $\mathbf{1 6 0}$ 


\subsubsection{Untersuchungen mit anderen Reduktionsmitteln}

Für die regioselektive Reduktion von 157 wurden weiter $\mathrm{LiBH}_{4}$ und $\mathrm{NaBH}_{4}$ als Reduktionsmittel untersucht. Beim stöchiometrischen Umsatz mit $\mathrm{LiBH}_{4}$ konnte bei $-78{ }^{\circ} \mathrm{C}$ keine Reaktion beobachtet werden. Weitere Erwärmung auf $0{ }^{\circ} \mathrm{C}$ und Raumtemperatur lieferte neben einer geringen Zersetzung keinen Umsatz liefern. Trotz überschüssiger Menge an $\mathrm{LiBH}_{4}$ wurde 141 nicht gebildet.
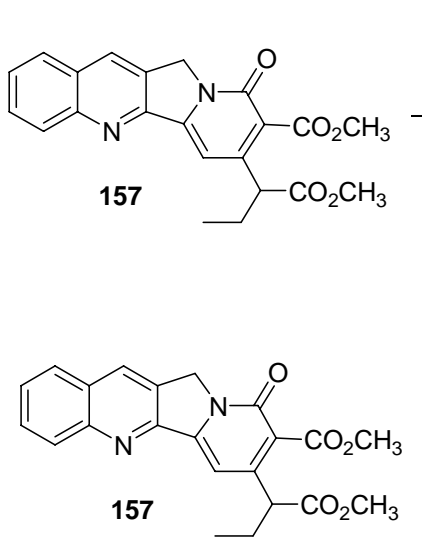

1.0 Äq. $\mathrm{LiBH}_{4}$ in THF, $-78^{\circ} \mathrm{C}, 30 \mathrm{~min}$

$0{ }^{\circ} \mathrm{C}, 70$ min und RT16 h; dann

$\mathrm{LiBH}_{4}$ (2.0 Äq.), $-78^{\circ} \mathrm{C}, 3 \mathrm{~h}$
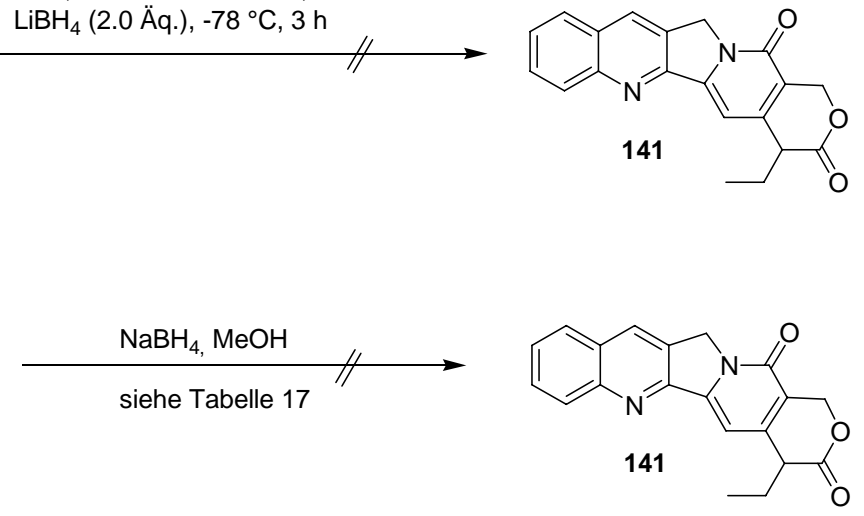

Schema 78. Untersuchung mit $\mathrm{LiBH}_{4}$ und $\mathrm{NaBH}_{4}$

Obwohl Esterfunktionalitäten normalerweise inert gegenüber $\mathrm{NaBH}_{4}$ sind, wurden versucht die am Pyridonring gebundene Estergruppe mit $\mathrm{NaBH}_{4} \mathrm{zu}$ reduzieren, da sie eine erhöhte Reaktivität aufweist. Selbst nach langer Reaktionszeit konnte jedoch nur das Edukt nachgewiesen. 


\begin{tabular}{ccc}
\hline Eintrag & Reaktionsbedingungen & Ergebnisse \\
\hline 1 & 1.0 Äq. 157, 8.0 Äq. $\mathrm{NaBH}_{4}$ in $\mathrm{MeOH}, \mathrm{RT}$, über Nacht & Edukt \\
2 & 1.0 Äq. 157, 8.0 Äq. $\mathrm{NaBH}_{4}$ in $\mathrm{MeOH}, 65^{\circ} \mathrm{C}, 2 \mathrm{~h}$ & Edukt und wenig \\
& & Zersetzung \\
\hline
\end{tabular}

Tabelle 17. Untersuchung mit $\mathrm{NaBH}_{4}$

\subsubsection{Differenzierung zwischen Methylester- und tert-Butylestergruppe}

Da die obige Differenzierung der Estergruppen nicht erfolgreich war, wurde die Einführung einer sperrigeren Estergruppe zur besseren Differenzierung geplant. Im Hinblick auf die geringere Effizienz des iso-Propylesters ${ }^{92}$ wurde ein tert-Butylester an dieser Stelle bevorzugt.

\subsubsection{Synthese des tert-Butylesters 162}

Zur Synthese der benötigten Verbindung 162 wurde zuerst versucht 161 herzustellen. Die

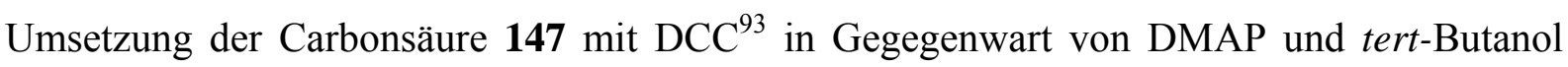
führte jedoch nicht zum gewünschten Produkt (siehe Tabelle 18). Ebenfalls nicht erfolgreich war die Reaktion von 147 mit 2,2,2-Trichloracetimidsäure-tert-butylester, ${ }^{94}$ hier konnte 161 neben Zersetzungsprodukten nur in geringen Mengen erhalten werden. Mit Di-tertbutyldicarbonat $\left(\mathrm{Boc}_{2} \mathrm{O}\right)^{95}$ in Gegenwart von DMAP ließ sich jedoch das gewünschte Produkt in 50\% Ausbeute isolieren. Weitere Versuche unter Einsatz von mehr Äquivalenten DMAP, Erwärmung $\left(55{ }^{\circ} \mathrm{C}\right.$ für $\left.30 \mathrm{~min}\right)$ und längerer Reaktionszeit führten nicht zu einer Verbesserung der Reaktion. Durch Dehydrierung mit DDQ konnte der tert-Butylester 162 in 38\% Ausbeute hergestellt werden. Es ist zu bemerken, dass 162 nach säulenchromatographischer Reinigung noch Verunreinigungen enthielt. 


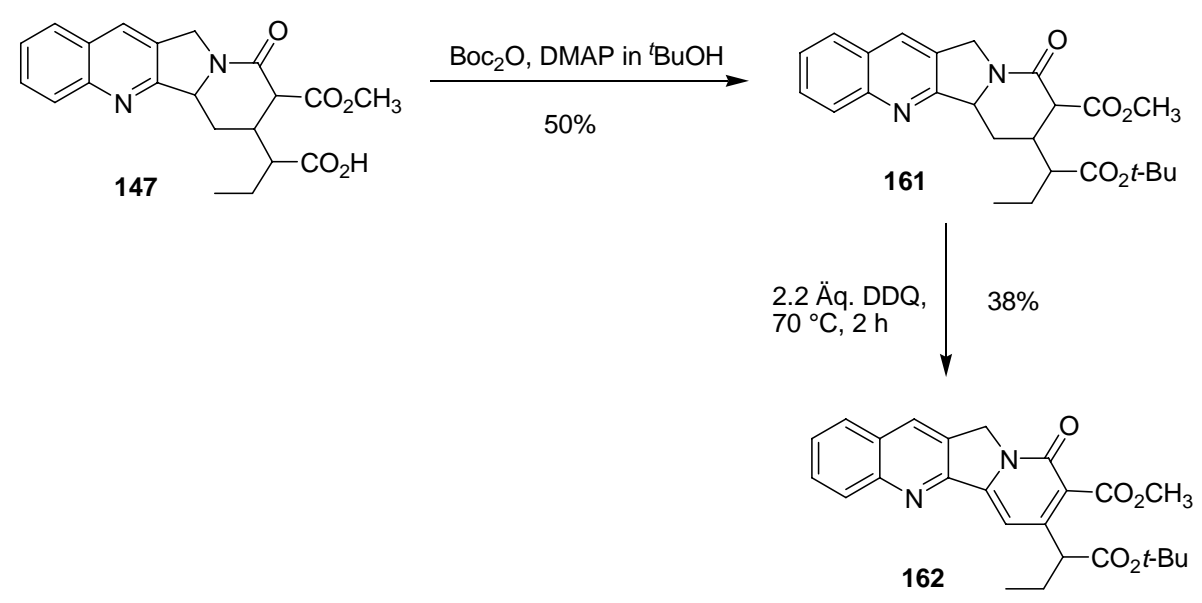

Schema 79. Synthese von 162

\begin{tabular}{|c|c|c|}
\hline Eintrag & Reaktionsbedingungen & Ausbeute (\%) \\
\hline 1 & $\begin{array}{l}\text { 1.0 Äq. 147,1.2 Äq. DCC, } 0.29 \text { Äq. DMAP, } 7.8 \text { Äq. } \\
\text { tert-Butanol in } \mathrm{CH}_{2} \mathrm{Cl}_{2}, 0^{\circ} \mathrm{C} \text { auf RT, } 4 \mathrm{~h}\end{array}$ & - \\
\hline 2 & $\begin{array}{l}1.0 \text { Äq. 147, } 1.6 \text { Äq. 2,2,2-Trichloracetimidsäure- } \\
\text { tert-Butylester, Cyclohexan/ } \mathrm{CH}_{2} \mathrm{Cl}_{2}, \mathrm{RT}, 16 \mathrm{~h}\end{array}$ & Spuren \\
\hline 3 & $\begin{array}{l}\text { 1.0 Äq. 147, } 2.0 \text { Äq. } \text { Boc }_{2} \mathrm{O}, 0.30 \text { Äq. DMAP in } \\
\text { tert-Butanol, RT, } 20 \mathrm{~h} \text {; dann } 8.7 \text { Äq. Boc }{ }_{2} \mathrm{O} \\
\text { innerhalb } 5 \mathrm{~h}\end{array}$ & $50 \%$ \\
\hline 4 & $\begin{array}{l}1.0 \text { Äq. 147, } 2.0 \text { Äq. } \mathrm{Boc}_{2} \mathrm{O}, 0.30 \text { Äq. DMAP in } \\
\text { tert-Butanol, RT, } 2.5 \mathrm{~h} \text {; dann } 1.1 \text { Äq. DMAP, } 55^{\circ} \mathrm{C} \\
\text { für } 30 \text { min, dann RT } 24 \mathrm{~h} \text {; dann } 16 \text { Äq. } \text { Boc }_{2} \mathrm{O}, \mathrm{RT} \\
24 \mathrm{~h}\end{array}$ & $49 \%$ \\
\hline
\end{tabular}

Tabelle 18. Synthese von 161

\subsubsection{Selektive Reduktion mit DIBAL-H und Lactonisierung}

Die Methylestergruppe von 162 ließ sich mit einer verdünnten Lösung von DIBAL-H in 1,2-Dimethoxyethan ${ }^{83}$ bei $-78{ }^{\circ} \mathrm{C}$ zu einem Gemisch von Aldehyd 163 und Alkohol 164 reduzieren. Die beiden Verbindungen ließen sich chromatographisch isolieren, allerdings zeigten ihre ${ }^{1}$ H-NMR-Spektren ähnliche Verunreinigungen wie die im Edukt. Das beste Ergebnis wurde erhalten, wenn das Gemisch ohne weitere Reinigung direkt mit $\mathrm{NaBH}_{4}$ in tert-Butanol umgesetzt und anschließend mit TFA behandelt wurde. Das gewünschte Lacton 
141 konnte in insgesamt 48\% Ausbeute dargestellt werden. Die obigen Verunreinigungen ließen sich an dieser Stelle bequem chromatographisch entfernen.

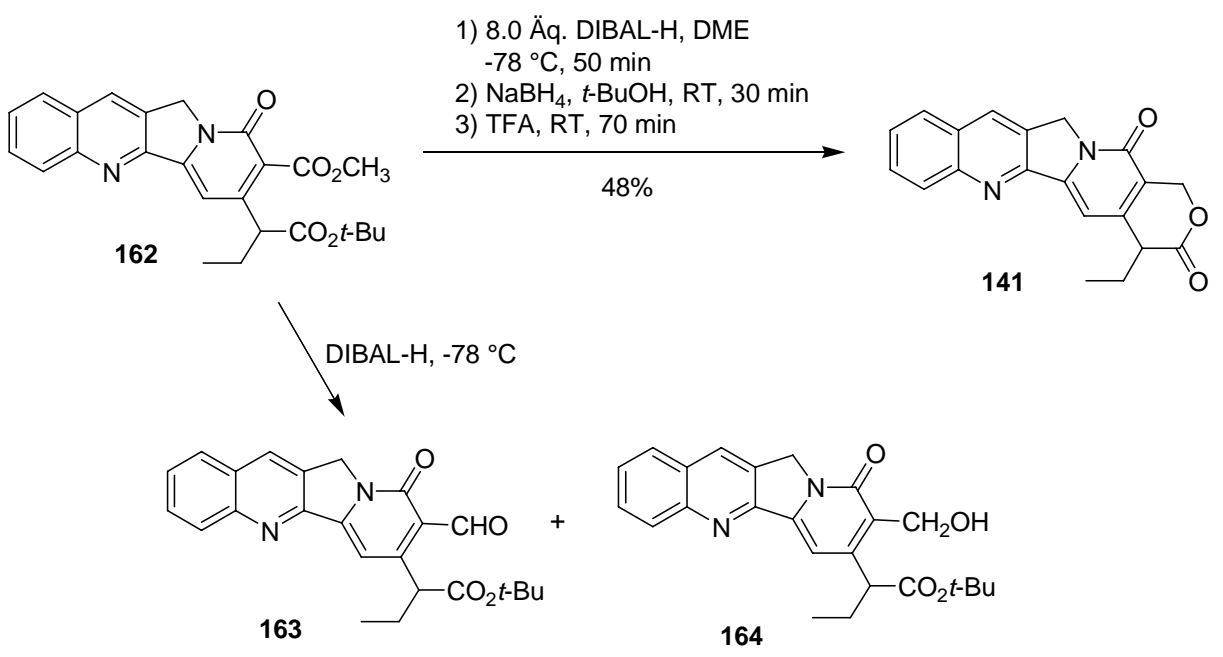

Schema 80. Synthese des Lactons 141 


\subsection{Weitere Modifizierungen}

Eine DDQ-vermittelte Dehydrierung von 62 konnte interessanterweise die Verbindung 165 liefern, welche auf die Enolform der Aldehydgruppe zurückzuführen war, die durch intramolekulare Umersterung zur Bildung des Lactonrings führte. Bei der Umsetzung von 165 mit $\mathrm{LiBH}_{4}$ oder $\mathrm{LiAlH}_{4}{ }^{96}$ wurde statt der Bildung von 166 eine rasche Zersetzung beobachtet.
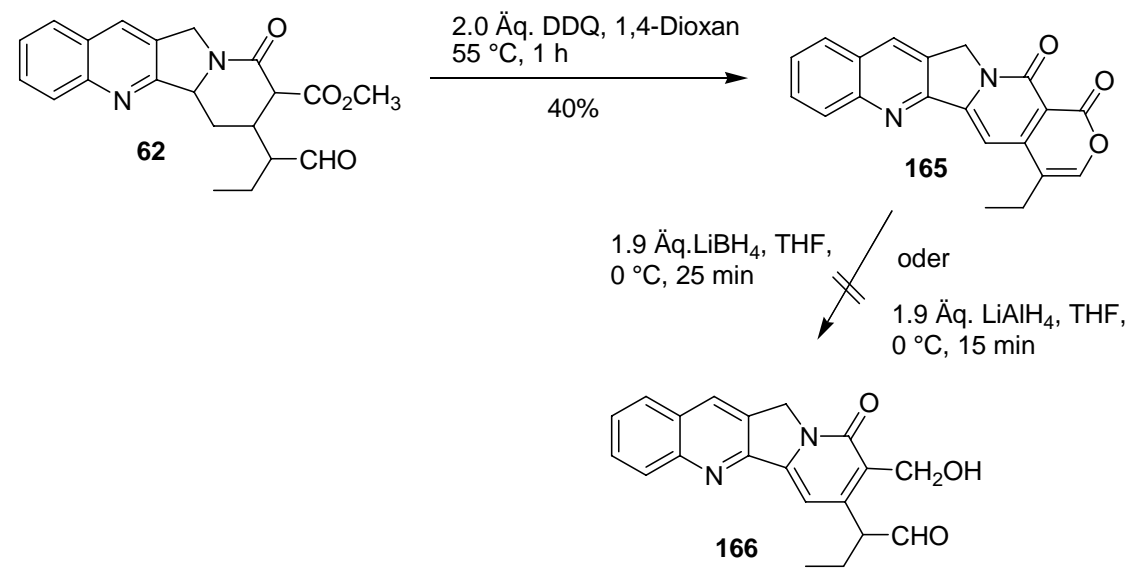

Schema 81. Synthese von 165

Obwohl den untersuchten Reaktionsbedingungen die Zielverbindung nicht erhalten wurde, sind die erhaltenen Informationen nützlich für enantioselektive Synthese von (20S)Camptothecin (siehe Schema 3). 


\section{ZUSAMMENFASSUNG}

Das pentacyclische Alkaloid Camptothecin besitzt eine ausgeprägte Zytotoxizität. Im Rahmen von Struktur-Wirkungs-Untersuchungen konnten als Analoga von Camptothecin die Verbindungen Irinotecan (Camptosar ${ }^{\circledR}$ ) and Topotecan (Hycamptin ${ }^{\circledR}$ ) hergestellt werden, die in der Krebstherapie eingesetzt werden. Im Hinblick auf die leichte Zugänglichkeit der Derivate durch synthetische Methoden ist eine effiziente bzw. industriell anwendbare Totalsynthese von Camptothecin von großer Bedeutung. Da die bislang publizierten Totalsynthesen von Camptothecin sehr viele Reaktionsschritte benötigen, ist es wünschenswert neue Zugänge zu Camptothecin mit hoher Gesamteffizienz zu entwickeln. In dieser Arbeit wird eine neuartige Totalsynthese von Camptothecin beschrieben. Hierzu wurde eine Domino-Knoevenagel-hetero-Diels-Alder-Reaktion zum Aufbau des Indolizinochinolin-Gerüstes verwendet.

Für den Aufbau des Indolizinochinolin-Gerüstes im Rahmen einer Domino-Knoevenagelhetero-Diels-Alder-Reaktion wurden der Enolether 64 und der Aldehyd 96, 114 bzw. 115 erforderlich. Die Synthese von 64 gelang durch Überführung von Butyraldehyd (67) in das Diethylacetal 70 und nachfolgende säurekatalytische Eliminierung von EtOH unter Verwendung von Sulfanilsäure.

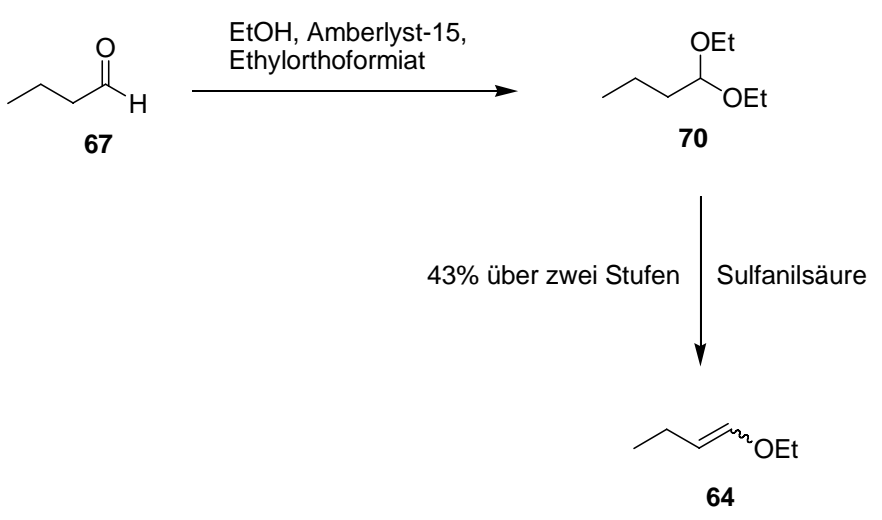

$E-/ Z$-Isomer $=0.35: 0.65$ 
Für die Synthese des Aldehyds 91 wurde Acetanilid (79) nach der Methode von Meth-Cohn umgesetzt (siehe Schema 83). Die anschließende Heck-Kupplung mit dem sterisch anspruchsvollen Liganden $\mathrm{P}(o \text {-tolyl })_{3}$ lieferte $\mathbf{8 1}$ in $65 \%$ Ausbeute liefern. Durch eine reduktive Aminierung von 81 mit $p$-Methoxybenzylamin bzw. Benzylamin unter Verwendung des Reduktionsmittels $\mathrm{NaBH}(\mathrm{OAc})_{3}$ in 1,2-Dichlorethan und nachfolgend intramolekulare 1,4-Michael-Addition wurden die Ester 100 und 93 in 92\% bzw. 80\% Ausbeute erhalten. Durch selektive DIBAL-H Reduktion und anschließende Umschützung unter Verwendung der $\mathrm{N}$-Dealkylierungsreagzien $\mathrm{Cbz}-\mathrm{Cl}$, Troc-Cl oder $\mathrm{ACE}-\mathrm{Cl}$ konnten ausgehend von dem $\mathrm{N}$ PMB-geschützten Ester 100 die gewünschten Aldehyde 96, 114 und 115 in 64\%, 68\% bzw. 54\% Ausbeute bereitgestellt werden. Im Vergleich dazu führte der N-Bn-geschützte Ester 93 unter ähnlichen Bedingungen zu den Aldehyden 96 und 114 in 63\% bzw. 46\% Ausbeute. Daraus lässt sich erkennen, dass die Verwendung von p-Methoxybenzylamin gegenüber Benzylamin vorteilhaft ist.

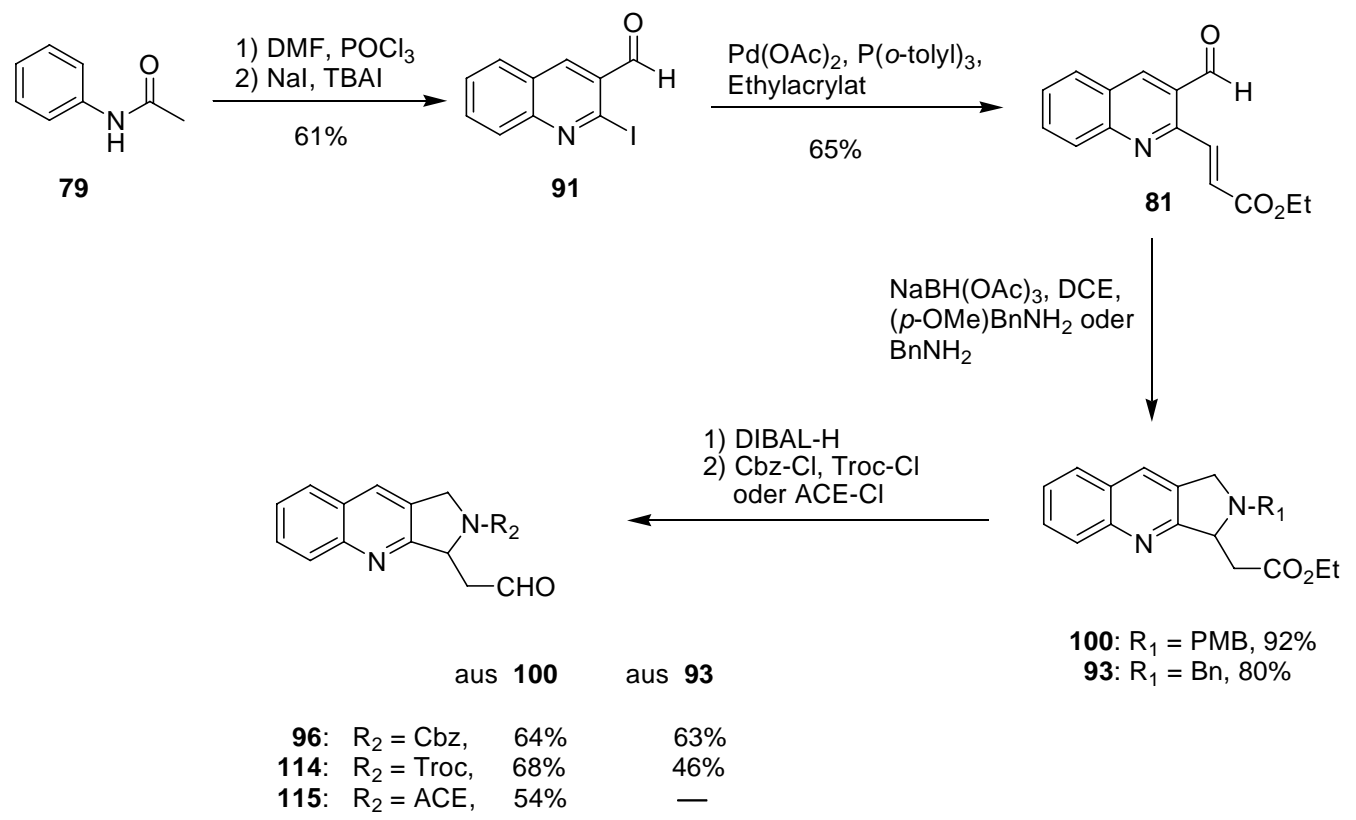

Schema 83. Synthese von Aldehyden

Die Domino-Knoevenagel-hetero-Diels-Alder-Reaktionen und anschließende Lactambildung wurden mit den Aldehyden 96, 114 und 115, Meldrum-Säure, und dem Dienophil 64 durchgeführt. Mit dem N-Troc-geschützten Aldehyd 114 ließ sich die Domino-Reaktion bei Raumtemperatur durchführen. Durch eine nachfolgende $\mathrm{Zn} / \mathrm{H}_{2} \mathrm{O}$-vermittelte $N$-TrocEntschützung unter Erwärmung mit Lactambildung konnte das Indolizinochinolin $\mathbf{6 1}$ in $41 \%$ Ausbeute dargestellt werden (siehe Schema 84). Der N-Cbz geschützte Aldehyd 96 wurde bei erhöhter Temperatur unter ansonsten gleichen Reaktionsbedingungen in der Domino- 
Reaktion umgesetzt, damit die anschließende Hydrogenolyse zu 61 sattfinden konnte. Das Indolizinochinolin 61 wurde somit in 59\% Ausbeute hergestellt werden. Mit dem N-ACEgeschützten Aldehyd 115 gelang zwar die Domino-Reaktion aber nicht die Lactambildung.<smiles>O=CCC1c2nc3ccccc3cc2CN1[N+](=O)[O-]</smiles>

114<smiles>O=CCC1c2nc3ccccc3cc2CN1C(=O)O</smiles>

96<smiles>CC(F)(F)N1Cc2cc3ccccc3nc2C1CC=O</smiles>

115

1) Meldrum-Säure, 64, RT

2) $\mathrm{Zn}, \mathrm{H}_{2} \mathrm{O}, 40^{\circ} \mathrm{C}$

$41 \%$

Meldrum-Säure, $64,50^{\circ} \mathrm{C}$

2) $\mathrm{H}_{2}, \mathrm{Pd} / \mathrm{C}$

$59 \%$

1) Meldrum-Säure, 64, RT

2) $\mathrm{MeOH}$ oder EtOH, Erwärmung

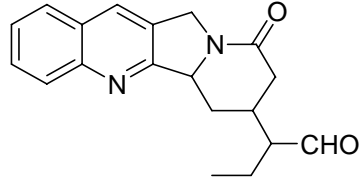

61

Schema 84. Synthese von 61 durch Domino-Reaktionen und anschließende Lactambildung

Durch die Oxidation mit $\mathrm{NaClO}_{2}$, Veresterung mit $\mathrm{TMS}-\mathrm{CHN}_{2}$ und anschließende Dehydrierung mit DDQ wurde 61 in den Methylester 20 in 78\% Ausbeute überführt. Die Bildung des Lactons 141 aus 20 nach der Danishefsky-Methode lieferte 20-Desoxycamptothecin 141 jedoch nur in einer geringen Ausbeute von 9\%. Die nachfolgende Hydroxylierung mit $\mathrm{O}_{2}, \mathrm{CuCl}_{2}$ und wäßrigem $\mathrm{Me}_{2} \mathrm{NH}$ vervollständigte die Synthese der Zielverbidung $( \pm)$-Camptothecin in $42 \%$ Ausbeute.

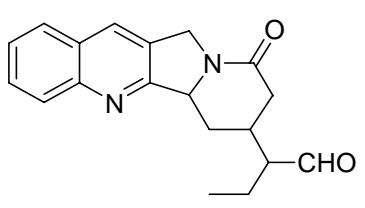

61

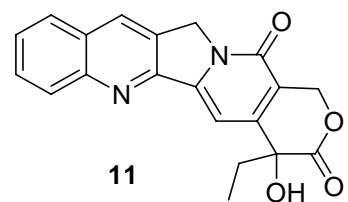

( \pm -Camptothecin
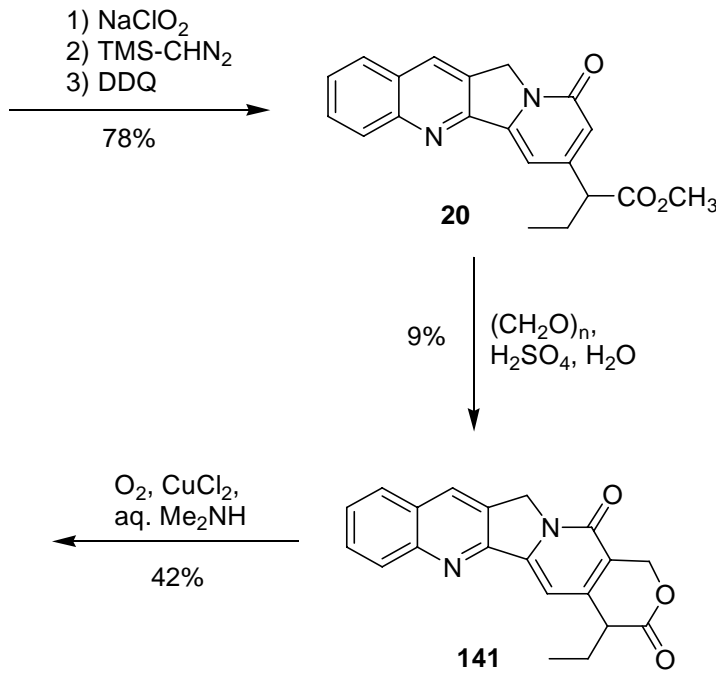
Durch eine Vierkomponenten-Domino-Knoevenagel-hetero-Diels-Alder-Reaktion von 96 bzw. 114 mit Meldrum-Säure, dem Enolether 64 und Methanol und anschließende Lactambildung konnte das Indolizinochinolingerüst 62 in 55\% bzw. 54\% Ausbeute dargestellt werden (siehe Schema 86). Oxidation, Veresterung mit (Boc) $)_{2} \mathrm{O}$ und Dehydrierung von 62 lieferten den Ester 162 in 19\% Ausbeute, der durch eine weitere Reduktion, TFAkatalytisierte Hydrolyse des tert-Butylesters und Lactonisierung in 20-Desoxycamptothecin 141 mit insgesamt $48 \%$ umgewandelt wurde.

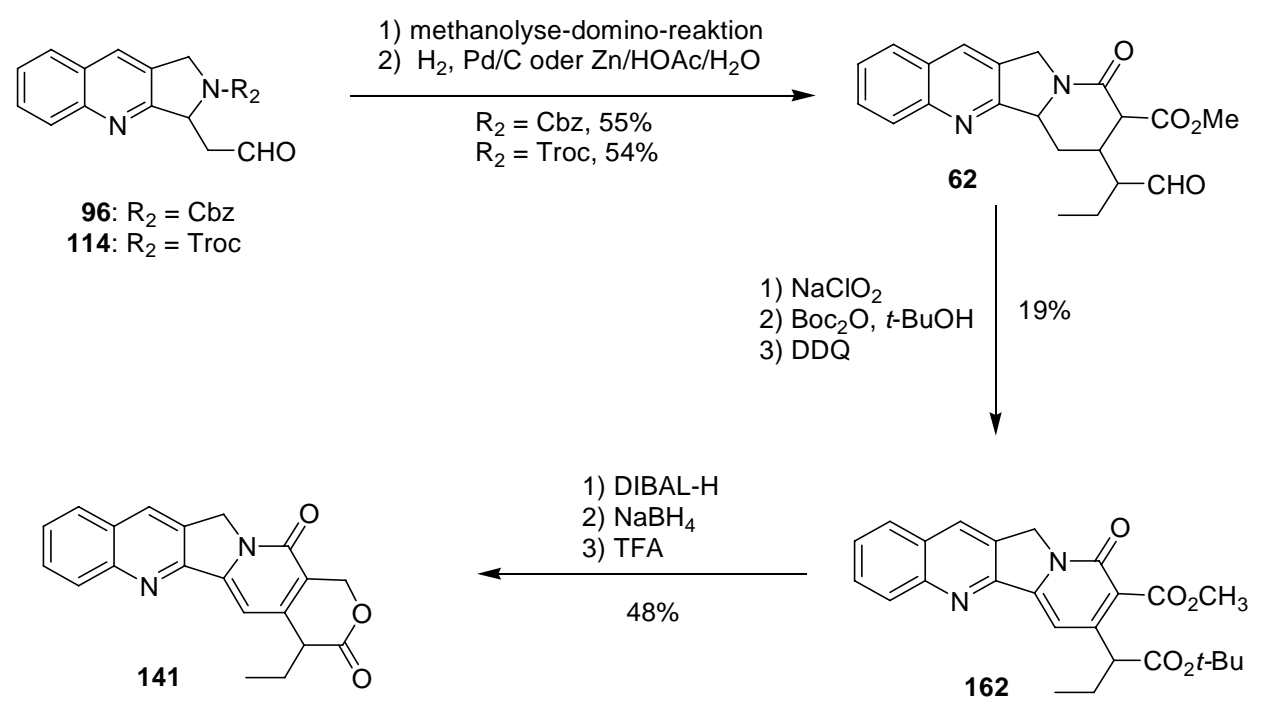

Schema 86. Synthese von 20-Desoxycamptothecin 141 durch Methanolyse-Domino-Reaktion

Ausgehend von 62 konnte auch der Diester 157 in 38\% Ausbeute über drei Stufen hergestellt werden. Die regioselektive Reduktion lieferte jedoch den Aldehyd 158 in nur 5\% Ausbeute (siehe Schema 87). Durch Reduktion und Cyclisierung von 158 mit $\mathrm{NaBH}_{4} / \mathrm{MeOH}$ wurde das Lactol 156 in quantitativer Ausbeute gebildet. Bei der Acetalschützung der Aldehydgruppe von 62 wurde das Acetal 154 über drei Stufen in 30\% Ausbeute erhalten. Die weitere Entschützung mit $\mathrm{LiBF}_{4}$ lieferte den Enolether 155 und das Lactol 156 in 60\% bzw. 40\% Ausbeute. 

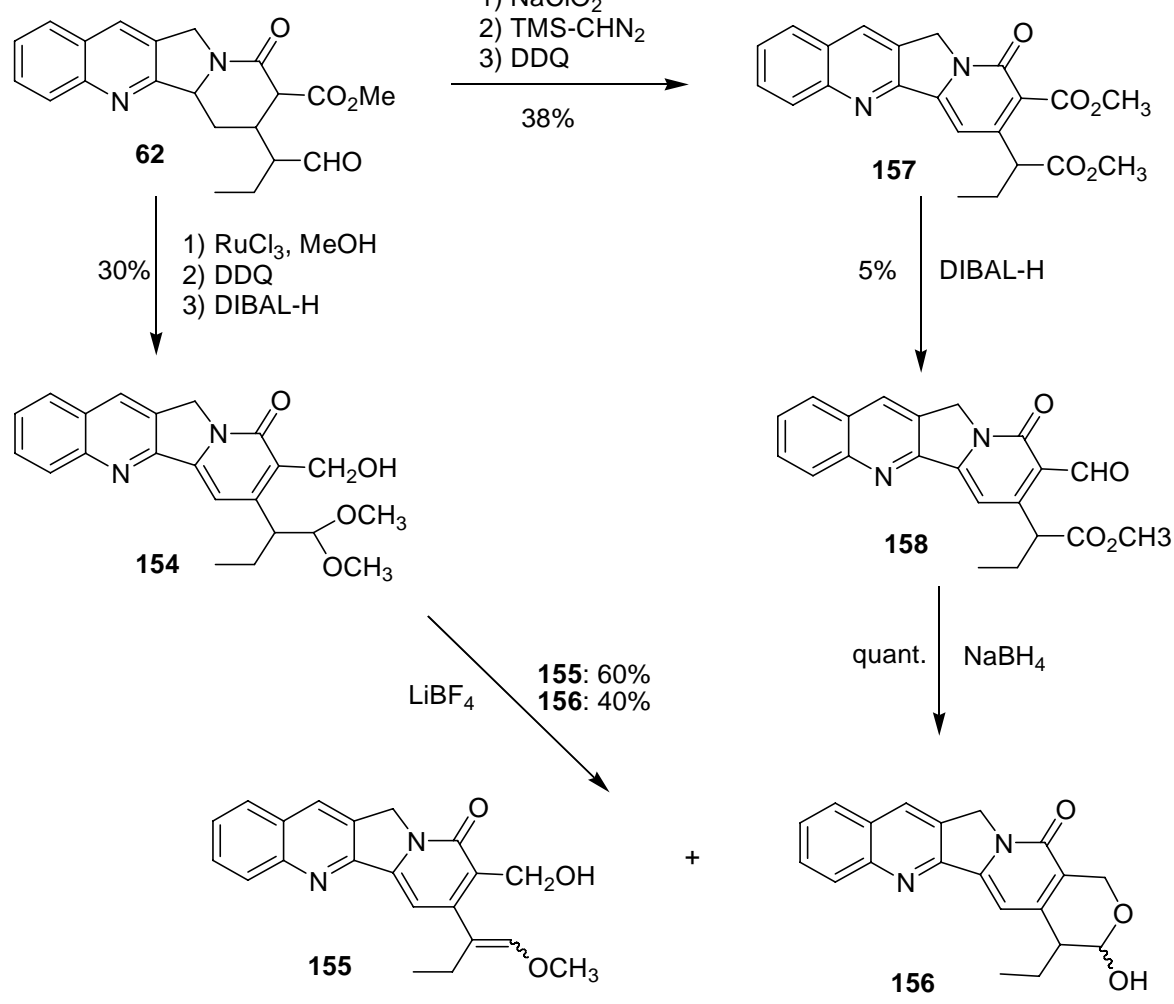

Schema 87. Synthese des Lactols 156

Zur enantioselektiven Einführung der Hydroxygruppe in 11 wurde 141 mit (+)-Camphorylsulfonyloxaziridin umgesetzt. Die Reaktion war jedoch nicht erfolgreicht, so wurde (20S)-Camptothecin (11) nur in Spuren gebildet werden.

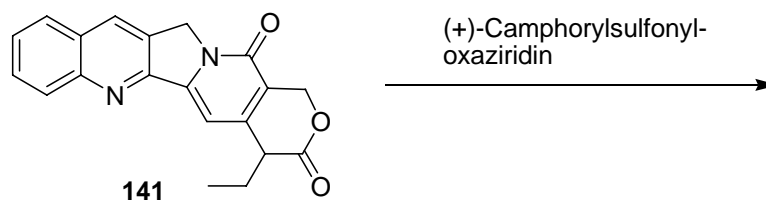

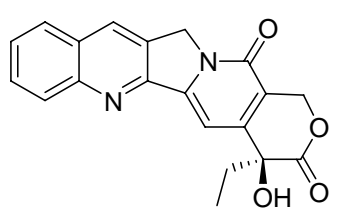

(20S)-11, Spuren

Schema 88. Versuch der enatioselektivern Einführung der Hydroxygruppe 


\section{EXPERIMENTELLER TEIL}

\section{Allgemeine Methoden}

Die Umsetzungen wurden soweit nötig in ausgeheizten Glasapparaturen unter einem leichten Argon-Überdruck durchgeführt. Die Lösungsmittel wurden entsprechend den üblichen Laboratoriumsmethoden getrocknet ${ }^{97}$ und destilliert. Käufliche Produkte wurden ohne weitere Reinigung eingesetzt. Das Entgasen von Lösungsmitteln geschah durch längeres Einleiten eines Argon-Gasstroms.

\subsection{Instrumentelle Analytik und verwendete Geräte}

Infrarotspektren: Die Infrarotspektren wurden mittels Modell Vector 22 der Firma Bruker aufgenommen. Feststoffe wurden als $\mathrm{KBr}$-Presslinge, Flüssigkeiten als Film zwischen $\mathrm{KBr}-$ Platten gemessen. Zur Kalibrierung diente die Polystyrolbande bei $1601 \mathrm{~cm}^{-1}$.

UV/VIS-Spektren: UV-Spektren wurden mit dem Modell Lambda 2 der Firma Perkin-Elmer aufgenommen. Der Messbereich umfasste eine Wellenlänge von 190-600 nm.

${ }^{1}$ H-NMR-Spektren: Modelle Mercury-200 (200 MHz), Mercury-300 und Unity-300 (je 300 MHz), Inova-500 (500 MHz) sowie Unity Inova-600 (600 MHz) der Firma Varian. Die chemischen Verschiebungen sind in Einheiten der $\delta$-Skala angegeben. Tetra-methylsilan $\left(\delta_{\mathrm{TMS}}=0.00\right.$ ppm) oder das angegebene Lösungsmittel dienten als interner Standard. Zur Kennzeichnung der Multiplizitäten der Signale werden folgende Abkürzungen verwendet: s (Singulett), d (Dublett), $\mathrm{t}$ (Triplett), q (Quartett), quint (Quintett), m (Multiplett), $\mathrm{m}_{\mathrm{c}}$ (zentriertes Multiplett), br (breites Signal). Die Spektren wurden in der Regel erster Ordnung entsprechend interpretiert. Die Kopplungs-konstanten $J$ sind in Hertz (Hz) angegeben.

${ }^{13}$ C-NMR-Spektren: Modelle Mercury-200 (50 MHz), Mercury-300 und Unity-300 (je 75 MHz), Inova-500 (125 MHz) sowie Unity Inova-600 (150 MHz). Als interner Standard diente Tetramethylsilan oder das angegebene Lösungsmittel. Die chemischen Verschiebungen sind den ${ }^{1}$ H-breitbandentkoppelten Spektren entnommen, die Multiplizitäten der Signale wurden in multiplett-selection-Experimenten (APT-Pulsfolge) bestimmt. Signale, die nicht eindeutig zugeordnet werden konnten, sind mit einem Stern $\left(^{*}\right)$ gekennzeichnet. 
Massenspektren: Die EI-, CI- und DCI-Spektren wurden mittels doppelfokussierendem Sektorfeld-Massenspektrometer MAT 95 der Firma Finnigan aufgenommen. ESI-Spektren wurden mit einem Triple-Stage-Quadrupol-Instrument TSQ 7000 oder einem Ion-TrapMassenspektrometer LCQ der Firma Finnigan aufgenommen. Angegeben werden die Quotienten aus Masse zu Ladung sowie in Klammern die relativen Intensitäten bezogen auf den Basispeak $(\mathrm{I}=100)$. Die Messung der ESI-HRMS-Spektren erfolgte an einem 7 TeslaFourier Transform Ion Cyclotron Resonance (FTICR)-Massenspekrometer APEX IV der Firma Bruker, das mit einer Apollo-Quelle der Firma Bruker und einer Spritzenpumpe 74900 series der Firma Cole-Parmer ausgestattet ist.

\subsection{Chromatographische Methoden}

Dünnschichtchromatographie (DC): Es wurden DC-Fertigfolien SIL G/UV254 und ALOX N/UV254 der Firma Macherey-Nagel \& Co. (Schichtdicke $0.25 \mathrm{~mm}$ ) verwendet. Angegebene $\mathrm{R}_{\mathrm{f}}$-Werte (Laufhöhe relativ zur Laufmittelfront) beziehen sich auf DC-Fertigfolien SIL G/UV254, wenn nichts anders bezeichnet wird. Neben der UV-Detektion dienten eine Vanillin-Schwefelsäure-Lösung (0.5 g Vanillin, $3 \mathrm{~mL}$ konz. Schwefelsäure, $85 \mathrm{~mL}$ Methanol und $10 \mathrm{~mL}$ Essigsäure) als Anfärbereagenzien.

Säulenchromatographie: Alle säulenchromatographischen Trennungen wurden mit Kieselgel 60 (Korngröße: 0.032-0.063 mm) der Firma Merck und Aluminiumoxid (aktiviert, neutral, 50-200 $\mu \mathrm{m})$ der Firma Acros durchgeführt. 


\section{Synthese von Dienophilen}

\section{$2.1 \quad$ 1,1-Dimethoxybutan (66)}

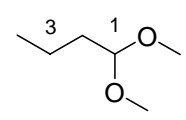

$\mathrm{Zu}$ einer Mischung von $\mathrm{RuCl}_{3} \cdot \mathrm{xH}_{2} \mathrm{O}(36 \% \mathrm{Ru}, 56 \mathrm{mg})$ in Methanol (40.5 mL, $1.00 \mathrm{~mol}$, 5.00 Äq.) bei $0{ }^{\circ} \mathrm{C}$ wurde Butyraldehyd (17.7 mL, $200 \mathrm{mmol}, 1.00$ Äq.) tropfenweise gegeben. Dann wurde das Reaktionsgemisch auf Raumtemperatur (RT) erwärmt und weiter für $17 \mathrm{~h}$ gerührt. Durch Zugabe von Wasser $(50 \mathrm{~mL})$ wurde die Reaktion beendet. Die wässrige Phase wurde mit $\mathrm{CH}_{2} \mathrm{Cl}_{2}(2 \times 200 \mathrm{~mL})$ extrahiert. Die vereinigten organischen Phasen wurden über $\mathrm{NaSO}_{4}$ getrocknet, filtriert und im Vakuum $\left(800 \mathrm{mbar} / 40{ }^{\circ} \mathrm{C}\right)$ eingeengt. Destillation des Rückstands lieferte das Acetal 66 (16.0 g, $0.135 \mathrm{mmol}, 68 \%$ ) als farblose Flüssigkeit.

Sdp.: $110-112^{\circ} \mathrm{C}$ (Normaldruck).

${ }^{1} \mathbf{H}-\mathbf{N M R}\left(200 \mathrm{MHz}, \mathrm{CDCl}_{3}\right): \delta=0.89\left(\mathrm{t},{ }^{3} \mathrm{~J}=7.2 \mathrm{~Hz}, 3 \mathrm{H}, 4-\mathrm{H}\right), 1.19-1.44$ (m, $\left.2 \mathrm{H}, 3-\mathrm{H}\right)$, $1.45-1.63(\mathrm{~m}, 2 \mathrm{H}, 2-\mathrm{H}), 3.28\left(\mathrm{~s}, 6 \mathrm{H}, 2 \times \mathrm{OCH}_{3}\right), 4.34\left(\mathrm{t},{ }^{3} \mathrm{~J}=5.8 \mathrm{~Hz}, 1 \mathrm{H}, 1-\mathrm{H}\right)$.

MS (CI, $\left.\mathrm{NH}_{3}\right): m / z(\%): 254.4$ (2) [2M+NH$]^{+}, 136.2$ (6) [M+NH$]^{+}, 89.1$ (100) [Base].

$\mathbf{C}_{6} \mathbf{H}_{14} \mathbf{O}_{2}$ (118.17).

\subsection{1,1-Diethoxybutan (70)}

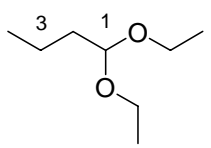

$\mathrm{Zu}$ einer Mischung von Amberlyst-15 (1.24 g), Ethylorthoformiat (55.3 mL, $0.333 \mathrm{mmol}$, 1.00 Äq.) in Ethanol (107 mL, $1.83 \mathrm{~mol}, 5.51$ Äq.) bei $0^{\circ} \mathrm{C}$ wurde Butyraldehyd (30.0 mL, $0.333 \mathrm{mmol}, 1.00$ Äq.) tropfenweise innerhalb $15 \mathrm{~min}$ gegeben. Dann wurde das Reaktionsgemisch bei RT für $18 \mathrm{~h}$ gerührt und anschließend bei $80^{\circ} \mathrm{C}$ für $3.5 \mathrm{~h}$ erhitzt. Nach 
Abkühlung auf RT wurde Amberlyst-15 abfiltriert und mit $\mathrm{Et}_{2} \mathrm{O}$ gewaschen. Die Mutterlauge wurde über $\mathrm{K}_{2} \mathrm{CO}_{3}$ getrocknet und filtriert. Nach Destillation des Rückstands wurde das Acetal 70 (31.8 g, 0.217 mmol, 65\%) als farblose Flüssigkeit erhalten.

Sdp.: $143.5-144{ }^{\circ} \mathrm{C}$ (Normaldruck).

${ }^{1}$ H-NMR (300 MHz, $\left.\mathrm{CDCl}_{3}\right): \delta=0.93\left(\mathrm{t},{ }^{3} \mathrm{~J}=7.4 \mathrm{~Hz}, 3 \mathrm{H}, 4-\mathrm{H}\right), 1.20\left(\mathrm{t},{ }^{3} \mathrm{~J}=7.1 \mathrm{~Hz}, 6 \mathrm{H}, 2 \times\right.$ $\left.\mathrm{OCH}_{2} \mathrm{CH}_{3}\right), 1.31-1.46(\mathrm{~m}, 2 \mathrm{H}, 3-\mathrm{H}), 1.54-1.65(\mathrm{~m}, 2 \mathrm{H}, 2-\mathrm{H}), 3.42-3.56(\mathrm{~m}, 2 \mathrm{H}, 2 \times$ $\left.\mathrm{OCH}_{\mathrm{a}} \mathrm{H}_{\mathrm{b}} \mathrm{CH}_{3}\right), 3.57-3.71\left(\mathrm{~m}, 2 \mathrm{H}, 2 \times \mathrm{OCH}_{\mathrm{a}} \mathrm{H}_{\mathrm{b}} \mathrm{CH}_{3}\right), 4.49\left(\mathrm{t},{ }^{3} \mathrm{~J}=5.8 \mathrm{~Hz}, 1 \mathrm{H}, 1-\mathrm{H}\right)$.

${ }^{13}$ C-NMR $\left(75 \mathrm{MHz}, \mathrm{CDCl}_{3}\right): \delta=13.86(\mathrm{C}-4), 15.26\left(2 \times \mathrm{OCH}_{2} \mathrm{CH}_{3}\right), 17.99(\mathrm{C}-3), 35.61$ (C-2), $60.70\left(2 \times \mathrm{OCH}_{2} \mathrm{CH}_{3}\right), 102.6(\mathrm{C}-1)$.

MS (CI, $\left.\mathrm{NH}_{3}\right): m / z(\%): 310.6(2)\left[2 \mathrm{M}+\mathrm{NH}_{4}\right]^{+}, 164.3(10)\left[\mathrm{M}+\mathrm{NH}_{4}\right]^{+}, 89.2$ (100) [Base].

$\mathrm{C}_{8} \mathrm{H}_{18} \mathrm{O}_{2}$ (146.23).

\subsection{1-Ethoxybut-1-en (64)}

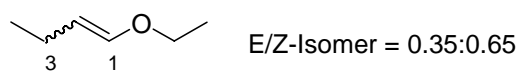

Ein Gemisch von 70 (20.0 mL, 16.2 g, 111 mmol, 1.00 Äq.) und Sulfanilsäure (250 mg, 1.44 mmol, $1.30 \mathrm{~mol} \%$ ) wurde auf $150^{\circ} \mathrm{C}$ erhitzt. Mit einer aufgesetzten Vigreux-kolonne $(10 \mathrm{~cm})$ wurde eine Fraktion in 40 min bei dem Siedepunkt von $64-73{ }^{\circ} \mathrm{C}$ in einem mit $\mathrm{Na}_{2} \mathrm{CO}_{3}$ beschichten Vorlagekolben gesammelt. Nach leichter Abkühlung wurde 70 (10.0 mL) in den Reaktionskolben eingespritzt. Es wurde dann auf $160{ }^{\circ} \mathrm{C}$ erhitzt, eine zweite Faktion bei dem Siedepunkt von $91{ }^{\circ} \mathrm{C}$ auf ähnliche Weise gesammelt. Danach wurde 70 (17.0 mL) gegeben, eine dritte Fraktion bei dem Siedepunkt von $74-96{ }^{\circ} \mathrm{C}$ gesammelt. Die vereinigten organischen Phasen wurden mit 5\%-wäßriger $\mathrm{NaHCO}_{3}$-Lösung $(4 \times 50 \mathrm{~mL})$ gewaschen, über $\mathrm{K}_{2} \mathrm{CO}_{3}$ getrocknet und filtriert. Destillation des Rückstands lieferte den Enolether 64 (16.7 g, $0.167 \mathrm{mmol}, 64 \%$ ) als farblose Flüssigkeit. 
Sdp.: $94-98^{\circ} \mathrm{C}$ (Normaldruck).

${ }^{1}$ H-NMR (300 MHz, $\mathrm{CDCl}_{3}$, E-Isomer/Z-Isomer = 0.35:0.65): $\delta=0.91-1.01(\mathrm{~m}, 3 \mathrm{H}, 4-\mathrm{H})$, 1.20-1.30 (m, $3 \mathrm{H}, \mathrm{OCH}_{2} \mathrm{CH}_{3}$ ), 1.93 (d von quint, ${ }^{3} \mathrm{~J}=7.4 \mathrm{~Hz},{ }^{4} \mathrm{~J}=1.2 \mathrm{~Hz}, 0.70 \mathrm{H}, 3-\mathrm{H}$, E-Isomer), 2.09 (d von quint, ${ }^{3} J=7.4 \mathrm{~Hz},{ }^{4} \mathrm{~J}=1.6 \mathrm{~Hz}, 1.30 \mathrm{H}, 3-\mathrm{H}, \mathrm{Z}$-Isomer), 3.69 (q, ${ }^{3} \mathrm{~J}=$ $7.1 \mathrm{~Hz}, 0.70 \mathrm{H}, \mathrm{OCH}_{2} \mathrm{CH}_{3}, E$-Isomer), 3.77 (q, ${ }^{3} \mathrm{~J}=7.1 \mathrm{~Hz}, 1.30 \mathrm{H}, \mathrm{OCH}_{2} \mathrm{CH}_{3}, \mathrm{Z}$-Isomer), $4.34\left(\mathrm{q},{ }^{3} \mathrm{~J}=6.8 \mathrm{~Hz}, 0.65 \mathrm{H}, 2-\mathrm{H}, \mathrm{Z}-\mathrm{Isomer}\right), 4.80$ (dt, ${ }^{3} \mathrm{~J}=12.6,7.0 \mathrm{~Hz}, 0.35 \mathrm{H}, 2-\mathrm{H}$, E-Isomer), 5.90 (dt, ${ }^{3} J=6.3 \mathrm{~Hz},{ }^{4} J=1.6 \mathrm{~Hz}, 0.65 \mathrm{H}, 1-\mathrm{H}, \mathrm{Z}$-Isomer), 6.22 (dt, ${ }^{3} J=12.6 \mathrm{~Hz}$, ${ }^{4} J=1.2 \mathrm{~Hz}, 0.35 \mathrm{H}, 1-\mathrm{H}$, E-Isomer).

${ }^{13}$ C-NMR (75 MHz, $\left.\mathrm{CDCl}_{3}\right): \delta=(Z$-Isomer, E-Isomer jeweils) 14.49, 14.78 (C-4), 15.23, $15.34\left(\mathrm{OCH}_{2} \mathrm{CH}_{3}\right), 17.34,21.12(\mathrm{C}-3), 67.43,64.50\left(\mathrm{OCH}_{2} \mathrm{CH}_{3}\right), 108.8,106.0(\mathrm{C}-2), 144.0$, $145.4(\mathrm{C}-1)$.

$\mathbf{C}_{6} \mathbf{H}_{12} \mathbf{O}(100.16)$.

\subsection{2-Oxobuttersäuremethylester (73)}<smiles>CCC(=O)C(C)=O</smiles>

$\mathrm{Zu}$ einer Mischung von $\alpha$-Ketobuttersäure (5.53 g, $54.2 \mathrm{mmol}, 1.00$ Äq.), Natrimsulfat (7.18 g, 50.5 mmol, 0.930 Äq.) in MeOH (60 mL, 1.48 mol, 27. 0 Äq.) bei RT wurde Chlortrimethylsilan $(0.700 \mathrm{~mL}, 5.54 \mathrm{mmol}, 0.100$ Äq.) gegeben. Es wurde weiter für $24 \mathrm{~h}$ gerührt. Nach Abfiltration von Natriumsulfat wurde die Mutterlauge konzentriert. Der Rückstand wurde in $\mathrm{Et}_{2} \mathrm{O}$ aufgenommen und abfiltriert. Nach Entfernung des Lösungsmittels im Vakuum (120 mbar, $40{ }^{\circ} \mathrm{C}$ ) erhielt man den Methylester 73 (5.48 g, $47.2 \mathrm{mmol}, 87 \%$ ) als farblose Flüssigkeit.

${ }^{1} \mathbf{H}-\mathbf{N M R}\left(200 \mathrm{MHz}, \mathrm{CDCl}_{3}\right): \delta=1.13\left(\mathrm{t},{ }^{3} J=7.2 \mathrm{~Hz}, 3 \mathrm{H}, \mathrm{CH}_{2} \mathrm{CH}_{3}\right), 2.87\left(\mathrm{q},{ }^{3} J=7.2 \mathrm{~Hz}, 2\right.$ $\left.\mathrm{H}, \mathrm{CH}_{2}\right), 3.87$ (s, $\left.3 \mathrm{H}, \mathrm{OCH}_{3}\right)$.

$\mathbf{C}_{5} \mathbf{H}_{8} \mathbf{O}_{3}(116.12)$. 


\subsection{2-Hydroxybuttersäuremethylester (74)}<smiles>CCC(O)C(C)=O</smiles>

Zu einer Lösung des Methylesters 73 (2.21 g, 19.0 mmol, 1.00 Äq.) in MeOH (100 mL) bei $0{ }^{\circ} \mathrm{C}$ wurde $\mathrm{NaBH}_{4}(1.44 \mathrm{~g}, 38.1 \mathrm{mmol}, 2.00$ Äq.) gegeben. Nach 15 min wurde die Reakton durch Zugabe von Wasser $(20 \mathrm{~mL})$, gesättiger $\mathrm{NH}_{4} \mathrm{Cl}$-Lösung $(60 \mathrm{~mL})$ und HCl-Lösung $(2 \mathrm{~N}$, $30 \mathrm{~mL})$ beendet. Die wässrige Phase wurde mit $\mathrm{CH}_{2} \mathrm{Cl}_{2}(5 \times 40 \mathrm{~mL})$ extrahiert. Die vereinigten organischen Phasen wurden über $\mathrm{MgSO}_{4}$ getrocknet und filtriert. Nach Entfernung des Lösungsmittels wurde der Alkohol 74 (1.72 g, 14.5 mmol, 76\%) als farblose Flüssigkeit gewonnen.

${ }^{1}$ H-NMR $\left(200 \mathrm{MHz}, \mathrm{CDCl}_{3}\right): \delta=0.94\left(\mathrm{t},{ }^{3} \mathrm{~J}=7.5 \mathrm{~Hz}, 3 \mathrm{H}, \mathrm{CH}_{2} \mathrm{CH}_{3}\right), 1.51-1.96(\mathrm{~m}, 2 \mathrm{H}$, $\left.\mathrm{CH}_{2}\right), 2.64(\mathrm{sbr}, 1 \mathrm{H}, \mathrm{OH}), 3.77\left(\mathrm{~s}, 3 \mathrm{H}, \mathrm{OCH}_{3}\right), 4.15\left(\mathrm{dd},{ }^{3} \mathrm{~J}=4.6 \mathrm{~Hz}, 6.6 \mathrm{~Hz}, 1 \mathrm{H}, 2-\mathrm{H}\right)$.

$\mathbf{C}_{5} \mathbf{H}_{10} \mathbf{O}_{3}(118.13)$.

\subsection{2-Benzyloxybuttersäure (78)}

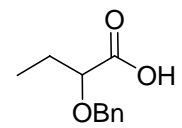

$\mathrm{Zu}$ einer Mischung von NaH (60\% in Paraffinöl, 2.40 g, 60.0 mmol, 2.00 Äq.) in THF (40 $\mathrm{mL}$ ) bei $0{ }^{\circ} \mathrm{C}$ wurde eine Lösung von Benzylalkohol (3.24 g, $30.0 \mathrm{mmol}, 1.00$ Äq.) in THF $(10 \mathrm{~mL})$ tropfenweise gegeben. Nach $30 \mathrm{~min}$ Rühren bei dieser Temperatur wurde die Reaktionsmischung auf RT erwärmt. Nach weiterem 30 min Rühren wurde die Reaktionsmischung auf $0{ }^{\circ} \mathrm{C}$ gekühlt und eine Lösung von DL-2-Brombuttersäure $(5.01 \mathrm{~g}, 30.0 \mathrm{mmol}$, 1.00 Äq.) in THF $(10 \mathrm{~mL})$ tropfenweise gegeben. Bei dieser Temperatur wurde die Reaktionsmischung für 30 min gerührt und danach bei $76^{\circ} \mathrm{C}$ für $6 \mathrm{~h}$ erhitzt. Nach Abkühlen auf $0{ }^{\circ} \mathrm{C}$ wurde die Reaktion durch Zugabe von wäßriger HCl-Lösung ( $2 \mathrm{~N}, 40 \mathrm{~mL}$ ) beendet. Die wäßrige Phase wurde mit EtOAc $(3 \times 80 \mathrm{~mL})$ extrahiert. Die vereinigten organischen Phasen wurden über $\mathrm{MgSO}_{4}$ getrocknet, filtriert und konzentriert. Man erhielt das Rohprodukt 
78 (6.75 g, 34.8 mmol, 116\%) als hellgelbes Öl, welches ohne weitere Reinigung für die weitere Stufe eingesetzt wurde.

MS (DCI, $\left.\mathrm{NH}_{3}\right): m / z(\%): 406.4(16)\left[2 \mathrm{M}+\mathrm{NH}_{4}\right]^{+}, 229.3(32)\left[\mathrm{M}+\mathrm{NH}_{3}+\mathrm{NH}_{4}\right]^{+}, 212.2(100)$

$\left[\mathrm{M}+\mathrm{NH}_{4}\right]^{+}$.

$\mathbf{C}_{11} \mathbf{H}_{14} \mathbf{O}_{3}(194.23)$.

\subsection{2-Benzyloxybuttersäuremethylester (75)}

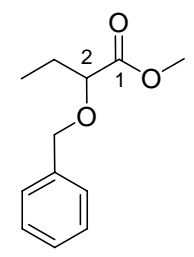

$\mathrm{Zu}$ einer Lösung von 78 (4.85 g, $25.0 \mathrm{mmol}$, 1.00 Äq.) in MeOH (127 mL) bei RT wurde Chlortrimethylsilan (6.95 mL, $55.0 \mathrm{mmol}, 2.20$ Äq.) tropfenweise gegeben. Bei dieser Temperatur wurde für $21 \mathrm{~h}$ gerührt. Nach Entfernung des Lösungsmittels wurde der Rückstand durch Säulenchromatographie auf Kieselgel (Pentan/EtOAc $=3: 1)$ gereinigt. Es wurde 75 (3.46 g, 16.6 mmol, 77\% über zwei Stufen) als farblose Flüssigkeit erhalten.

$\mathbf{R}_{\mathbf{f}}=0.70($ Petan $/$ EtOAc $=6: 1)$.

UV $\left(\mathrm{CH}_{3} \mathrm{CN}\right): \lambda_{\max }(\lg \varepsilon)=205.5 \mathrm{~nm}(3.922), 257.0$ (2.368).

IR (KBr): $\widetilde{v}=2969 \mathrm{~cm}^{-1}(\mathrm{C}-\mathrm{H}), 1750(\mathrm{C}=\mathrm{O}), 1496,1455\left(\mathrm{CH}_{3}, \mathrm{CH}_{2}\right), 1203,1139(\mathrm{C}-\mathrm{O}), 739$, $698\left(\mathrm{CH}_{3}, \mathrm{CH}_{2}\right)$.

${ }^{1} \mathbf{H}-\mathbf{N M R}\left(300 \mathrm{MHz}, \mathrm{CDCl}_{3}\right): \delta=0.97\left(\mathrm{t},{ }^{3} \mathrm{~J}=7.5 \mathrm{~Hz}, 3 \mathrm{H}, \mathrm{CH}_{2} \mathrm{CH}_{3}\right), 1.71-1.87(\mathrm{~m}, 2 \mathrm{H}$, $\left.\mathrm{CH}_{2} \mathrm{CH}_{3}\right), 3.74\left(\mathrm{~s}, 3 \mathrm{H}, \mathrm{OCH}_{3}\right), 3.89\left(\mathrm{t},{ }^{3} \mathrm{~J}=6.9 \mathrm{~Hz}, 1 \mathrm{H}, 2-\mathrm{H}\right), 4.42\left(\mathrm{~d},{ }^{2} \mathrm{~J}=11.7 \mathrm{~Hz}, 1 \mathrm{H}\right.$, $\left.\mathrm{OCH}_{\mathrm{a}} \mathrm{H}_{\mathrm{b}}\right), 4.70\left(\mathrm{~d},{ }^{2} J=11.7 \mathrm{~Hz}, 1 \mathrm{H}, \mathrm{OCH}_{\mathrm{a}} H_{\mathrm{b}}\right), 7.24-7.41(\mathrm{~m}, 5 \mathrm{H}, 5 \times \mathrm{Ph}-H)$.

${ }^{13}$ C-NMR $\left(150 \mathrm{MHz}, \mathrm{CDCl}_{3}\right): \delta=9.65\left(\mathrm{CH}_{2} \mathrm{CH}_{3}\right), 26.16\left(\mathrm{CH}_{2} \mathrm{CH}_{3}\right), 51.70\left(\mathrm{OCH}_{3}\right), 72.17$ $\left(\mathrm{OCH}_{2}\right), 79.16(\mathrm{C}-2), 127.73(p-\mathrm{Ph}-\mathrm{C}), 127.89(2 \times m-\mathrm{Ph}-\mathrm{C}), 128.30(2 \times o-\mathrm{Ph}-\mathrm{C}), 137.52$ (ipso-Ph-C), $173.19\left(\mathrm{CO}_{2} \mathrm{Me}\right)$.

MS (DCI, $\left.\mathrm{NH}_{3}\right): m / z(\%): 434.5(2)\left[2 \mathrm{M}+\mathrm{NH}_{4}\right]^{+}, 226.3(100)\left[\mathrm{M}+\mathrm{NH}_{4}\right]^{+}$.

$\mathbf{C}_{12} \mathbf{H}_{16} \mathbf{O}_{3}(208.25)$. 


\section{Synthese von Aldehyden}

\subsection{2-Chlor-3-chinolincarbaldehyd (80)}

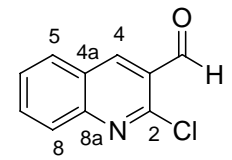

Phosphoroxychlorid (96.0 mL, $1.05 \mathrm{~mol}, 7.00$ Äq.) wurde innerhalb $100 \mathrm{~min}$ tropfenweise zu auf $0{ }^{\circ} \mathrm{C}$ gekühltem DMF (28.8 mL, $375 \mathrm{mmol}, 2.50$ Äq.) gegeben. Die Reaktionsmischung wurde dann auf RT erwärmt und für 20 min gerührt. Dann wurde Acetanilid (20.2 g, 150 mmol, 1.00 Äq.) gegeben und die Rektionsmischung auf $80^{\circ} \mathrm{C}$ für $16.5 \mathrm{~h}$ erhitzt, wobei die Inentempearatur bei $75^{\circ} \mathrm{C}$ blieb. Nach Abkühlung auf RT wurde die Reaktionsmischung in Eiswasser $(700 \mathrm{~mL})$ gegossen, wobei sich gelbe Niederschläge bildeten. Nach 30 min Rühren unter Eiskühlung wurde der Feststoff filtriert, mit Wasser $(300 \mathrm{~mL})$ gewaschen und im Hochvakuum getrocknet. Es wurde der Aldehyd 80 (18.3 g, 95.5 mmol, 64\%) als gelben Feststoff erhalten.

$\mathbf{R}_{\mathbf{f}}=0.51(\operatorname{Petan} / \operatorname{EtOAc}=4: 1)$.

${ }^{1}$ H-NMR $\left(300 \mathrm{MHz} \mathrm{CDCl}_{3}\right): \delta=7.65\left(\mathrm{~m}_{\mathrm{c}}, 1 \mathrm{H}, 6-\mathrm{H}\right), 7.88\left(\mathrm{~m}_{\mathrm{c}}, 1 \mathrm{H}, 7-\mathrm{H}\right), 7.98\left(\mathrm{~d},{ }^{3} \mathrm{~J}=8.2\right.$ $\mathrm{Hz}, 1 \mathrm{H}, 5-\mathrm{H}), 8.06\left(\mathrm{~d},{ }^{3} \mathrm{~J}=8.5 \mathrm{~Hz}, 1 \mathrm{H}, 8-\mathrm{H}\right), 8.74$ (s, $\left.1 \mathrm{H}, 4-\mathrm{H}\right), 10.55$ (s, $\left.1 \mathrm{H}, \mathrm{CHO}\right)$.

${ }^{13}$ C-NMR (125 MHz, $\left.\mathrm{CDCl}_{3}\right): \delta=126.3\left(\mathrm{C}-3^{*}\right), 126.4\left(\mathrm{C}-4 \mathrm{a}^{*}\right), 128.1$ (C-6), $128.5(\mathrm{C}-8)$, 129.6 (C-5), 133.5 (C-7), 140.2 (C-4), 149.5 (C-2), 150.0 (C-8a), 189.0 (CHO).

$\mathbf{C}_{10} \mathbf{H}_{6}$ CINO (191.61). 


\section{$3.2 \quad$ 2-Iod-3-chinolincarbaldehyd (91)}

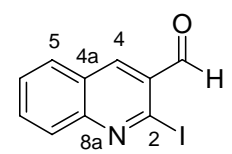

Zu einer Mischung von NaI (9.01 g, 60.0 mmol, 4.19 Äq.) in $\mathrm{CH}_{3} \mathrm{CN}$ (50 mL) bei RT wurde 80 (2.75 g, $14.3 \mathrm{mmol}, 1.00$ Äq.) und TBAI (44.0 mg, $0.120 \mathrm{mmol}, 0.840 \mathrm{~mol} \%$ ) gegeben. Nach Erwärmung auf $40{ }^{\circ} \mathrm{C}$ wurde $\mathrm{HCl}\left(2 \mathrm{M}\right.$ in $\left.\mathrm{Et}_{2} \mathrm{O}, 0.20 \mathrm{~mL}\right)$ gegeben. Es wurde bei dieser Temperatur für $22 \mathrm{~h}$ gerührt. Danach wurde die Reaktionsmischung im Vakuum konzentriert. Der Rückstand wurde mit Wasser $(30 \mathrm{~mL})$ und gesättiger $\mathrm{Na}_{2} \mathrm{CO}_{3}$-Lösung $(4 \mathrm{~mL})$ versetzt und die wässrige Phase mit $\mathrm{CH}_{2} \mathrm{Cl}_{2}(2 \times 80 \mathrm{~mL})$ extrahiert. Die vereinigten organischen Phasen wurden über $\mathrm{MgSO}_{4}$ getrocknet, filtriert und im Vakuum konzentriert. Man erhielt 91 (3.90 g, $13.8 \mathrm{mmol}, 96 \%)$ als gelben Feststoff.

$\mathbf{R}_{\mathbf{f}}=0.51(\operatorname{Petan} /$ EtOAc $=4: 1)$.

${ }^{1} \mathbf{H}-\mathbf{N M R}\left(300 \mathrm{MHz}, \mathrm{CDCl}_{3}\right): \delta=7.66\left(\mathrm{~m}_{\mathrm{c}}, 1 \mathrm{H}, 6-\mathrm{H}\right), 7.86\left(\mathrm{~m}_{\mathrm{c}}, 1 \mathrm{H}, 7-\mathrm{H}\right), 7.96\left(\mathrm{~d},{ }^{3} \mathrm{~J}=8.4\right.$ Hz, $1 \mathrm{H}, 5-\mathrm{H}), 8.09$ (d, $\left.{ }^{3} \mathrm{~J}=8.7 \mathrm{~Hz}, 1 \mathrm{H}, 8-\mathrm{H}\right), 8.54$ (s, $\left.1 \mathrm{H}, 4-\mathrm{H}\right), 10.27$ (s, $\left.1 \mathrm{H}, \mathrm{CHO}\right)$.

${ }^{13}$ C-NMR (75 MHz, $\left.\mathrm{CDCl}_{3}\right): \delta=120.5$ (C-2), 126.4 (C-4a), 128.3 (C-6), 128.6 (C-3), 128.8 (C-8), 129.7 (C-5), 133.4 (C-7), 138.7 (C-4), 151.5 (C-8a), 194.6 (CHO).

MS (70 eV, EI): $m / z(\%)=284.1(9), 283.1(100)[\mathrm{M}]^{+}, 156.1$ (51) $[\mathrm{M}-\mathrm{I}]^{+}, 128.1$ (43)

$[\mathrm{M}-\mathrm{CO}-\mathrm{I}]^{+}$.

\section{$\mathrm{C}_{10} \mathrm{H}_{6} \mathrm{INO}(283.07)$.}




\section{3 (E)-3-(3-Formylchinolin-2-yl)acrylsäureethylester (81)}

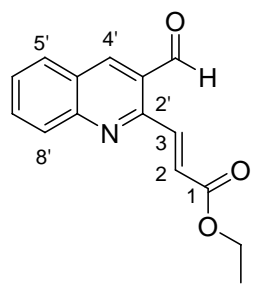

Ein Mischung von 91 (10.4 g, 36.6 mmol, 1.00 Äq.), Ethylacrylat (8.50 mL, 78.0 mmol, 2.13 Äq.) in DMF wurde bei RT durch ein Argon-Ballon für 50 min entgast. Dann wurde Natriumacetat (4.48 g, $54.8 \mathrm{mmol}, 1.50$ Äq.) und TBAI (720 mg, $1.95 \mathrm{mmol}, 5.33 \mathrm{~mol} \%$ ) zugegeben. Nach Erwärmung auf $40{ }^{\circ} \mathrm{C}$ wurde $\mathrm{Pd}(\mathrm{OAc})_{2}(175 \mathrm{mg}, 0.780 \mathrm{mmol}, 2.13 \mathrm{~mol} \%)$ und $\mathrm{P}(\text { o-tolyl })_{3}$ (475 mg, $1.56 \mathrm{mmol}, 4.33 \mathrm{~mol} \%$ ) hinzufügt. Die Reaktionsmischung wurde bei $120{ }^{\circ} \mathrm{C}$ für $12 \mathrm{~h}$ erhitzt und dann im Vakuum konzentriert. Nach Abkühlung auf $0{ }^{\circ} \mathrm{C}$ wurde der Rückstand with $\mathrm{CH}_{2} \mathrm{Cl}_{2}(400 \mathrm{~mL})$, Wasser $(200 \mathrm{~mL})$ und gesättiger $\mathrm{NaHCO}_{3}$ Lösung $(50 \mathrm{~mL})$ versetzt. Nach Phasentrennung wurde die organische Phase mit Wasser $(2 \times$ $100 \mathrm{~mL}$ ) gewaschen, über $\mathrm{Na}_{2} \mathrm{SO}_{4}$ getrocknet, filtriert. Nach Entfernung des Lösungsmittels wurde der Rückstand durch Säulenchromatographie auf Kieselgel $\left(\mathrm{CH}_{2} \mathrm{Cl}_{2} / \mathrm{EtOAc}=50: 1\right)$ gereinigt. Man erhielt 81 (6.07 g, 23.8 mmol, 65\%) als gelben Feststoff.

$\mathbf{R}_{\mathbf{f}}=0.36\left(\mathrm{CH}_{2} \mathrm{Cl}_{2} / \mathrm{EtOAc}=50: 1\right)$ oder $0.42(\operatorname{Petan} /$ EtOAc $=4: 1)$.

UV $\left(\mathrm{CH}_{3} \mathrm{CN}\right): \lambda_{\max }(\lg \varepsilon)=208.0 \mathrm{~nm}$ (4.293), 270.5 (4.677).

IR $(\mathrm{KBr}): \widetilde{v}=1714 \mathrm{~cm}^{-1}(\mathrm{C}=\mathrm{O}), 1370,1294\left(\mathrm{CH}_{2}\right.$ und $\left.\mathrm{CH}_{3}\right), 988(\mathrm{CH}=\mathrm{CH})$.

${ }^{1}$ H-NMR (300 MHz, d ${ }_{6}$-DMSO): $\delta=1.29\left(\mathrm{t},{ }^{3} J=7.1 \mathrm{~Hz}, 3 \mathrm{H}, \mathrm{CH}_{3}\right), 4.24\left(\mathrm{q},{ }^{3} J=7.1 \mathrm{~Hz}\right.$, $\left.2 \mathrm{H}, \mathrm{OCH}_{2}\right), 7.09$ (d, $\left.{ }^{3} \mathrm{~J}=15.4 \mathrm{~Hz}, 1 \mathrm{H}, 2-\mathrm{H}\right), 7.72\left(\mathrm{~m}_{\mathrm{c}}, 1 \mathrm{H}, 6^{\prime}-\mathrm{H}\right), 7.94\left(\mathrm{~m}_{\mathrm{c}}, 1 \mathrm{H}, 7^{\prime}-\mathrm{H}\right), 8.07$ $\left(\mathrm{d},{ }^{3} \mathrm{~J}=8.7 \mathrm{~Hz}, 1 \mathrm{H}, 8^{\prime}-\mathrm{H}\right), 8.15\left(\mathrm{~d},{ }^{3} \mathrm{~J}=8.1 \mathrm{~Hz}, 1 \mathrm{H}, 5^{\prime}-\mathrm{H}\right), 8.58\left(\mathrm{~d},{ }^{3} \mathrm{~J}=15.4 \mathrm{~Hz}, 1 \mathrm{H}, 3-\mathrm{H}\right)$, 8.96 (s, $\left.1 \mathrm{H}, 4^{\prime}-\mathrm{H}\right), 10.27$ (s, $\left.1 \mathrm{H}, \mathrm{CHO}\right)$.

${ }^{13}$ C-NMR (75 MHz, d 6 -DMSO): $\delta=14.02\left(\mathrm{CH}_{3}\right), 60.31\left(\mathrm{OCH}_{2}\right), 125.0$ (C-2), 126.8 (C-4'a), 127.4 (C-3'), 128.3 (C-6'), 129.1 (C-8'), 129.2 (C-5'), 133.2 (C-7'), 139.6 (C-3), 144.4 (C-4'), 148.1 (C-8'a), 150.2 (C-2'), $165.6\left(\mathrm{CO}_{2}\right), 192.4$ (CHO).

MS (70 eV, EI): $m / z(\%)=255.1(6)[\mathrm{M}]^{+}, 182.1(100)\left[\mathrm{M}-\mathrm{CO}_{2} \mathrm{C}_{2} \mathrm{H}_{5}\right]^{+}$.

$\mathrm{C}_{15} \mathbf{H}_{13} \mathrm{NO}_{3}$ (255.27). 


\section{4 (2-Benzyl-2,3-dihydro-1H-pyrrolo[3,4-b]chinolin-3-yl)-essigsäure-} ethylester (93)

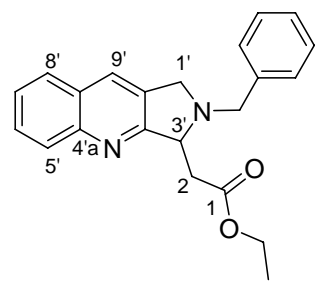

Zur einer Mischung von Benzylamin (0.680 mL, 6.22 mmol, 1.51 Äq.), $\mathrm{NaBH}(\mathrm{OAc})_{3}(1.32 \mathrm{~g}$, 6.22 mmol, 1.50 Äq.) in 1,2-Dichlorethan (DCE, $14 \mathrm{~mL}$ ) bei RT wurde eine Lösung von 81 (1.05 g, $4.11 \mathrm{mmol}, 1.00$ Äq.) in DCE (26 mL) innerhalb $100 \mathrm{~min}$ zugetropft. Dann wurde weiter für $6 \mathrm{~h}$ gerührt. Durch Zugabe von gesättiger $\mathrm{Na}_{2} \mathrm{CO}_{3}$-Lösung $(10 \mathrm{~mL})$ und Wasser (10 $\mathrm{mL})$ wurde die Reaktion beendet. Die wäßrige Phase wurde mit $\mathrm{CH}_{2} \mathrm{Cl}_{2}(2 \times 40 \mathrm{~mL})$ extrahiert. Die vereinigten organischen Phasen wurden über $\mathrm{Na}_{2} \mathrm{SO}_{4}$ getrocknet und filtriert. Nach Entfernung des Lösungsmittels wurde der Rückstand durch Säulenchromatographie auf Aluminiumoxid (neutral) $($ Pentan/EtOAc $=10: 1)$ gereinigt. Man erhielt $93(1.14 \mathrm{~g}, 3.29$ mmol, 80\%) als braun-gelbes Öl.

$\mathbf{R}_{\mathbf{f}}=0.36(\operatorname{Petan} / \operatorname{EtOAc}=6: 1)$.

UV $\left(\mathrm{CH}_{3} \mathrm{CN}\right): \lambda_{\max }(\lg \varepsilon)=193.0 \mathrm{~nm}$ (4.615), 209.5 (4.770), 230.5 (4.582), 234.0 (4.533), 264.5 (3.627), 306.5 (3.610), 319.5 (3.687).

IR (KBr): $\widetilde{v}=1730 \mathrm{~cm}^{-1}(\mathrm{C}=\mathrm{O}), 1243\left(\mathrm{CH}_{2}\right), 739\left(\mathrm{CH}_{2}\right)$.

${ }^{1} \mathbf{H}-\mathrm{NMR}\left(300 \mathrm{MHz}, \mathrm{CDCl}_{3}\right): \delta=1.20\left(\mathrm{t},{ }^{3} J=7.1 \mathrm{~Hz}, 3 \mathrm{H}, \mathrm{CH}_{3}\right), 2.98\left(\mathrm{dd},{ }^{2} J=15.7 \mathrm{~Hz},{ }^{3} J=\right.$ $\left.5.9 \mathrm{~Hz}, 1 \mathrm{H}, 2-\mathrm{H}_{\mathrm{a}}\right), 3.27\left(\mathrm{dd},{ }^{2} J=15.7 \mathrm{~Hz},{ }^{3} \mathrm{~J}=4.9 \mathrm{~Hz}, 1 \mathrm{H}, 2-\mathrm{H}_{\mathrm{b}}\right), 3.64-3.75\left(\mathrm{~m}, 2 \mathrm{H}, 1\right.$ ' $-\mathrm{H}_{\mathrm{a}}$, $\left.\mathrm{CH}_{\mathrm{a}} \mathrm{H}_{\mathrm{b}} \mathrm{Ph}\right), 4.11-4.23\left(\mathrm{~m}, 3 \mathrm{H}, \mathrm{OCH}_{2}, 1\right.$ ' $\left.-\mathrm{H}_{\mathrm{b}}\right), 4.31\left(\mathrm{~d},{ }^{2} J=13.5 \mathrm{~Hz}, 1 \mathrm{H}, \mathrm{CH}_{\mathrm{a}} H_{\mathrm{b}} \mathrm{Ph}\right), 4.54\left(\mathrm{~m}_{\mathrm{c}}\right.$, $1 \mathrm{H}, 3^{\prime}$-H), 7.23-7.51 (m, $\left.6 \mathrm{H}, 5 \times \mathrm{Ph}-H, 7^{\prime}-\mathrm{H}\right), 7.62\left(\mathrm{~m}_{\mathrm{c}}, 1 \mathrm{H}, 6^{\prime}-\mathrm{H}\right), 7.71\left(\mathrm{~d},{ }^{3} J=8.1 \mathrm{~Hz}, 1\right.$ H, 8'-H), 7.77 (s, $\left.1 \mathrm{H}, 9^{\prime}-\mathrm{H}\right), 8.05$ (d, $\left.{ }^{3} \mathrm{~J}=8.3 \mathrm{~Hz}, 1 \mathrm{H}, 5^{\prime}-\mathrm{H}\right)$.

${ }^{13}$ C-NMR $\left(75 \mathrm{MHz}, \mathrm{CDCl}_{3}\right): \delta=14.10\left(\mathrm{CH}_{3}\right), 38.83(\mathrm{C}-2), 55.48\left(\mathrm{C}-1{ }^{\prime}\right), 58.60\left(\mathrm{CH}_{2} \mathrm{Ph}\right)$, $60.31\left(\mathrm{OCH}_{2}\right), 65.15$ (C-3'), 125.9 (C-7'), 127.1 (p-Ph-C), 127.6 (C-9'a), 127.6 (C-8'), 128.3 ( $2 \times$ m-Ph-C), 128.6 (C-6', C-9'), 128.7 (2 × o-Ph-C), 129.0 (C-5'), 131.1 (C-8'a), 138.6 (ipso-Ph-C), 147.8 (C-4'a), 164.2 (C-3'a), $172.0\left(\mathrm{CO}_{2}\right)$.

MS (70 eV, EI): $m / z(\%)=346.3(23)[\mathrm{M}]^{+}, 259.2(100)\left[\mathrm{M}-\mathrm{CH}_{2} \mathrm{CO}_{2} \mathrm{C}_{2} \mathrm{H}_{5}\right]^{+}, 255.2$ (79) $\left[\mathrm{M}-\mathrm{CH}_{2} \mathrm{C}_{6} \mathrm{H}_{5}\right]^{+}, 91.1(82)\left[\mathrm{CH}_{2} \mathrm{C}_{6} \mathrm{H}_{5}\right]^{+}$.

$\mathbf{C}_{22} \mathbf{H}_{22} \mathbf{N}_{2} \mathbf{O}_{2}(346.42)$. 


\section{5 (2-(4-Methoxybenzyl)-2,3-dihydro-1H-pyrrolo[3,4-b]chinolin-3-yl)-} essigsäureethylester (100)

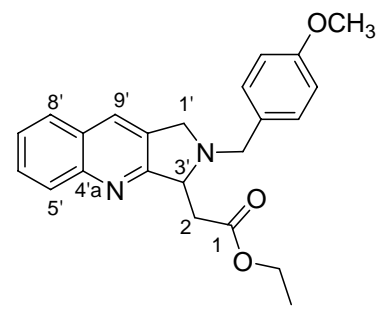

Zur einer Mischung von 4-Methoxybenzylamin (1.80 g, 12.4 mmol, 1.50 Äq.), NaBH(OAc) 3 (2.80 g, $13.2 \mathrm{mmol}, 1.60$ Äq.) in 1,2-Dichlorethan (DCE, $30 \mathrm{~mL}$ ) bei RT wurde eine Lösung von 81 (2.11 g, $8.26 \mathrm{mmol}, 1.00$ Äq.) in DCE (45 mL) innerhalb 30 min zugetropft. Dann wurde weiter für $5 \mathrm{~h}$ gerührt. Durch Zugabe von gesättiger $\mathrm{Na}_{2} \mathrm{CO}_{3}$-Lösung $(30 \mathrm{~mL})$ und Wasser $(30 \mathrm{~mL})$ wurde die Reaktion beendet. Die wäßrige Phase wurde mit $\mathrm{CH}_{2} \mathrm{Cl}_{2}(2 \times$ $50 \mathrm{~mL}$ ) extrahiert. Die vereinigten organischen Phasen wurden über $\mathrm{Na}_{2} \mathrm{SO}_{4}$ getrocknet und filtriert. Nach Entfernung des Lösungsmittels wurde der Rückstand durch Säulenchromatographie auf Aluminiumoxid (neutral) $($ Pentan/EtOAc $=6: 1)$ gereinigt. Man erhielt 100 (2.86 g, 7.60 mmol, 92\%) als gelbes Öl, welches bei Kühlung zum gelben Feststoff wurde.

$\mathbf{R}_{\mathbf{f}}=0.20\left(\right.$ Petan $/$ EtOAc $=8: 1, \mathrm{Al}_{2} \mathrm{O}_{3}$-Typ N$)$ oder $0.45($ Petan $/$ EtOAc $=4: 1$ Kieselgel $)$.

UV $\left(\mathrm{CH}_{3} \mathrm{CN}\right): \lambda_{\max }(\lg \varepsilon)=195.5 \mathrm{~nm}$ (4.754), 209.5 (4.754), 230.5 (4.691), 274.5 (3.751), 306.5 (3.626), 319.5 (3.706).

IR $(\mathrm{KBr}): \widetilde{v}=1730 \mathrm{~cm}^{-1},(\mathrm{C}=\mathrm{O}), 1514\left(\mathrm{CH}_{3}\right), 1248\left(\mathrm{CH}_{2}\right), 1161,1034(\mathrm{C}-\mathrm{O}), 749\left(\mathrm{CH}_{2}\right)$.

${ }^{1}$ H-NMR (300 MHz, $\left.\mathrm{CDCl}_{3}\right): \delta=1.20\left(\mathrm{t},{ }^{3} J=7.2 \mathrm{~Hz}, 3 \mathrm{H}, \mathrm{CH}_{2} \mathrm{CH}_{3}\right), 2.97\left(\mathrm{dd},{ }^{2} J=15.7 \mathrm{~Hz}\right.$, $\left.{ }^{3} J=5.9 \mathrm{~Hz}, 1 \mathrm{H}, 2-\mathrm{H}_{\mathrm{a}}\right), 3.25\left(\mathrm{dd},{ }^{2} J=15.7 \mathrm{~Hz},{ }^{3} J=4.8 \mathrm{~Hz}, 1 \mathrm{H}, 2-\mathrm{H}_{\mathrm{b}}\right), 3.61-3.75(\mathrm{~m}, 2 \mathrm{H}$, $\left.1^{\prime}-\mathrm{H}_{\mathrm{a}}, \mathrm{CH}_{\mathrm{a}} \mathrm{H}_{\mathrm{b}} \mathrm{Ph}(p-\mathrm{OMe})\right), 3.80\left(\mathrm{~s}, 3 \mathrm{H}, \mathrm{OCH}_{3}\right), 4.11-4.27\left(\mathrm{~m}, 4 \mathrm{H}, \mathrm{CO}_{2} \mathrm{CH}_{2} \mathrm{CH}_{3}, 1\right.$ '- $\mathrm{H}_{\mathrm{b}}$, $\left.\mathrm{CH}_{\mathrm{a}} \mathrm{H}_{\mathrm{b}} \mathrm{Ph}(p-\mathrm{OMe})\right), 4.52\left(\mathrm{~m}_{\mathrm{c}}, 1 \mathrm{H}, \quad 3{ }^{\prime}-\mathrm{H}\right), 6.84-6.92(\mathrm{~m}, 2 \mathrm{H}, 2 \times m-\mathrm{Ph}(p-\mathrm{OMe})-\mathrm{H})$, 7.28-7.36 (m, 2 H, 2 × o-Ph(p-OMe)-H), $7.46\left(\mathrm{~m}_{\mathrm{c}}, 1 \mathrm{H}, 7^{\prime}-\mathrm{H}\right), 7.63\left(\mathrm{~m}_{\mathrm{c}}, 1 \mathrm{H}, 6\right.$ '-H), 7.73 (d, $\left.{ }^{3} J=8.0 \mathrm{~Hz}, 1 \mathrm{H}, 8^{\prime}-\mathrm{H}\right), 7.79$ (s, $\left.1 \mathrm{H}, 9^{\prime}-\mathrm{H}\right), 8.04$ (d, $\left.{ }^{3} J=8.5 \mathrm{~Hz}, 1 \mathrm{H}, 5^{\prime}-\mathrm{H}\right)$.

${ }^{13}$ C-NMR (125 MHz, $\left.\mathrm{CDCl}_{3}\right): \delta=14.13\left(\mathrm{CH}_{2} \mathrm{CH}_{3}\right), 38.84(\mathrm{C}-2), 55.19\left(\mathrm{OCH}_{3}\right), 55.38(\mathrm{C}-1$ '), $57.92\left(\mathrm{CH}_{2} \mathrm{Ph}\right), 60.32\left(\mathrm{OCH}_{2}\right), 65.07$ (C-3’), 113.7 (2 × m-Ph(p-OMe)-C), $125.9\left(\mathrm{C}-7^{\prime}\right), 127.6$ (C-9’a, C-8'), 128.6 (C-6', C-9'), 129.0 (C-5’), 129.9 (2 × o-Ph(p-OMe)-C), 130.6 (ipso$\mathrm{Ph}(p-\mathrm{OMe})-\mathrm{C}), 131.1$ (C-8'a), 147.8 (C-4'a), 158.8 (p-Ph(p-OMe)-C), 164.3 (C-3'a), 172.0 $\left(\mathrm{CO}_{2}\right)$. 
MS (70 eV, EI): $m / z(\%)=376.4(6)[\mathrm{M}]^{+}, 255.3(34)\left[\mathrm{M}-\mathrm{CH}_{2} \mathrm{Ph}\left(p-\mathrm{OCH}_{3}\right)\right]^{+}, 121.2(100)$ $\left[\mathrm{CH}_{2} \mathrm{Ph}\left(p-\mathrm{OCH}_{3}\right)\right]^{+}$.

$\mathrm{C}_{23} \mathrm{H}_{24} \mathbf{N}_{2} \mathbf{O}_{3}$ (376.45).

\section{6 (2-Benzyl-2,3-dihydro-1H-pyrrolo[3,4-b]chinolin-3-yl)-acetaldehyd} (94)

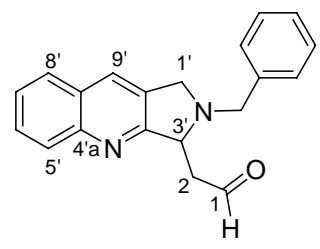

$\mathrm{Zu}$ einer Lösung von $93(0.11 \mathrm{~g}, 0.31 \mathrm{mmol}, 1.0 \mathrm{Äq}$. $)$ in $\mathrm{CH}_{2} \mathrm{Cl}_{2}(1.5 \mathrm{~mL})$ bei $-78^{\circ} \mathrm{C}$ wurde DIBAL-H (1.0 M in Hexan, 0.81 mL, 2.6 Äq.) zugetropft. Es wurde bei dieser Temperatur für 100 min gerührt. Die Reaktion wurde durch Zugabe von Methanol (1.0 mL) beendet. Die Mischung wurde auf RT erwärmt und mit gestättiger Natrium-Kalium-Tartrat-Lösung ( $5 \mathrm{~mL})$ und Wasser $(5 \mathrm{~mL})$ versetzt. Die wäßrige Phase wurde mit EtOAc $(2 \times 20 \mathrm{~mL})$ extrahiert. Die vereinigten organischen Phasen wurden mit gestättiger NaCl-Lösung gewaschen, über $\mathrm{Na}_{2} \mathrm{SO}_{4}$ getrocknet und filtriert. Nach Entfernung des Lösungsmittels wurde der Rückstand durch Säulenchromatographie auf Kieselgel (Pentan/EtOAc/ $/ \mathrm{NEt}_{3}=4: 1: 0.05 \rightarrow$ Pentan/EtOAc $/ \mathrm{MeOH}=1: 1: 0.2)$ gereinigt. Man erhielt den Aldehyd $94(55 \mathrm{mg}, 0.18 \mathrm{mmol}, 59 \%)$ als braunes Ö1.

$\mathbf{R}_{\mathbf{f}}=0.57($ Petan/EtOAc $=2: 1$, Zersetzung auf Kieselgel $)$.

UV $\left(\mathrm{CH}_{3} \mathrm{CN}\right): \lambda_{\max }(\lg \varepsilon)=207.5 \mathrm{~nm}$ (4.700), 209.0 (4.701), 230.5 (4.540), 234.0 (4.500), 306.5 (3.619), 319.5 (3.665).

IR (KBr): $\widetilde{v}=2800 \mathrm{~cm}^{-1}$ (Aldehyd C-H), $1722(\mathrm{C}=\mathrm{O}), 1315\left(\mathrm{CH}_{2}\right), 751,701\left(\mathrm{CH}_{2}\right)$.

${ }^{1}$ H-NMR $\left(600 \mathrm{MHz}, \mathrm{CDCl}_{3}\right): \delta=3.13\left(\mathrm{ddd},{ }^{2} J=16.7 \mathrm{~Hz},{ }^{3} \mathrm{~J}=5.2 \mathrm{~Hz},{ }^{3} \mathrm{~J}=3.7 \mathrm{~Hz}, 1 \mathrm{H}\right.$, 2- $\left.\mathrm{H}_{\mathrm{a}}\right), 3.29\left(\mathrm{ddd},{ }^{2} J=16.7 \mathrm{~Hz},{ }^{3} J=3.9 \mathrm{~Hz},{ }^{3} J=1.1 \mathrm{~Hz}, 1 \mathrm{H}, 2-\mathrm{H}_{\mathrm{b}}\right), 3.64\left(\mathrm{~d},{ }^{2} J=12.9 \mathrm{~Hz}, 1 \mathrm{H}\right.$, $\left.\mathrm{CH}_{\mathrm{a}} \mathrm{H}_{\mathrm{b}} \mathrm{Ph}\right), 3.71\left(\mathrm{~d},{ }^{2} J=13.8 \mathrm{~Hz}, 1 \mathrm{H}, 1^{\prime}-\mathrm{H}_{\mathrm{a}}\right), 4.18\left(\mathrm{~d},{ }^{2} J=13.8 \mathrm{~Hz}, 1 \mathrm{H}, 1\right.$ ' $\left.-\mathrm{H}_{\mathrm{b}}\right), 4.27\left(\mathrm{~d},{ }^{2} \mathrm{~J}=\right.$ $\left.12.9 \mathrm{~Hz}, 1 \mathrm{H}, \mathrm{CH}_{\mathrm{a}} \mathrm{H}_{\mathrm{b}} \mathrm{Ph}\right), 4.45\left(\mathrm{~m}_{\mathrm{c}}, 1 \mathrm{H}, 3^{\prime}-\mathrm{H}\right), 7.26-7.37$ (m, $\left.5 \mathrm{H}, 5 \times \mathrm{Ph}-H\right), 7.49\left(\mathrm{~m}_{\mathrm{c}}, 1 \mathrm{H}\right.$, 
7'-H), 7.65 (m $\left.\mathrm{m}_{\mathrm{c}}, 1 \mathrm{H}, 6^{\prime}-\mathrm{H}\right), 7.75\left(\mathrm{~d},{ }^{3} \mathrm{~J}=8.1 \mathrm{~Hz}, 1 \mathrm{H}, 8^{\prime}-\mathrm{H}\right), 7.83$ (s, $\left.1 \mathrm{H}, 9^{\prime}-\mathrm{H}\right), 8.05$ (d, ${ }^{3} J=$ $\left.8.6 \mathrm{~Hz}, 1 \mathrm{H}, 5^{\prime}-\mathrm{H}\right), 9.70\left(\mathrm{dd},{ }^{3} \mathrm{~J}=3.7 \mathrm{~Hz},{ }^{3} \mathrm{~J}=1.1 \mathrm{~Hz}, 1 \mathrm{H}, \mathrm{CHO}\right)$.

${ }^{13}$ C-NMR (125 MHz, $\left.\mathrm{CDCl}_{3}\right): \delta=45.45(\mathrm{C}-2), 55.52\left(\mathrm{C}-1\right.$ ') $57.99\left(\mathrm{CH}_{2} \mathrm{Ph}\right), 63.86(\mathrm{C}-3$ '), 126.2 (C-7'), 127.4 (p-Ph-C), 127.7 (C-9'a, C-8'), 128.5 (2 × m-Ph-C), 128.8 ( $2 \times$ o-Ph-C), 128.9 (C-5'), 129.0 (C-9', C-6'), 131.2 (C-8'a), 138.1 (ipso-Ph-C), 147.9 (C-4'a), 163.8 (C-3'a), 201.7 (CHO).

MS (70 eV, EI): $m / z(\%)=302.3(4)[\mathrm{M}]^{+}, 259.2(100)\left[\mathrm{M}-\mathrm{CH}_{2} \mathrm{CHO}\right]^{+}, 91.1$ (86) $\left[\mathrm{CH}_{2} \mathrm{C}_{6} \mathrm{H}_{5}\right]^{+}$.

$\mathrm{C}_{20} \mathbf{H}_{18} \mathbf{N}_{2} \mathbf{O}$ (302.37).

\section{7 (2-Benzyloxycarbonyl-2,3-dihydro-1H-pyrrolo[3,4-b]chinolin-3-yl)- acetaldehyd (96)}

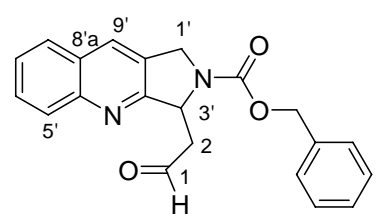

Zu einer Lösung von 100 (2.15 g, $5.71 \mathrm{mmol}, 1.00$ Äq.) in $\mathrm{CH}_{2} \mathrm{Cl}_{2}(60 \mathrm{~mL})$ bei $-78^{\circ} \mathrm{C}$ wurde DIBAL-H (1.0 M in Dichlormethan, $6.00 \mathrm{~mL}, 1.10$ Äq.) zugetropft. Dann wurde für $40 \mathrm{~min}$ gerührt. Bei dieser Temperatur wurde DIBAL-H (1.0 M in Dichlormethan, $0.800 \mathrm{~mL}, 0.140$ Äq.) weiter gegeben. Nach 40 min Rühren wurde die Reaktion durch Zugabe von Methanol $(2.0 \mathrm{~mL})$ und Wasser $(10 \mathrm{~mL})$ beendet. Die Mischung wurde auf $0{ }^{\circ} \mathrm{C}$ erwärmt und $\mathrm{mit}_{\mathrm{Et}} \mathrm{O}$ $(200 \mathrm{~mL})$ versetzt. Die organische Phase wurden nachfolgend mit wäßriger NaOH-Lösung $(1 \mathrm{~N}, 2 \times 60 \mathrm{~mL})$, Wasser $(60 \mathrm{~mL})$ und gestättiger $\mathrm{NaCl}$-Lösung gewaschen, über $\mathrm{Na}_{2} \mathrm{SO}_{4}$ getrocknet, filtriert und im Vakuum konzentriert. Das erhaltene dunkle Öl (1.90 g) wurde mit $\mathrm{CH}_{3} \mathrm{CN}$ (40 mL) und $\mathrm{K}_{2} \mathrm{CO}_{3}(160 \mathrm{mg}, 1.16 \mathrm{mmol}, 0.203$ Äq.) versetzt. $\mathrm{Zu}$ dieser Mischung bei $0{ }^{\circ} \mathrm{C}$ wurde $\mathrm{Cbz}-\mathrm{Cl}(1.20 \mathrm{~mL}, 8.44 \mathrm{mmol}, 1.48$ Äq.) gegeben. Es wurde für $30 \mathrm{~min}$ bei dieser Tempeartur und dann bei RT für $13 \mathrm{~h}$ gerührt. Danach wurde Cbz-Cl $(1.20 \mathrm{~mL}, 8.44$ mmol, 1.48 Äq.) weiter gegeben und die Reaktionsmischung für $3 \mathrm{~h}$ gerührt. $\mathrm{K}_{2} \mathrm{CO}_{3}$ wurde abfiltriert und mit EtOAc gewaschen. Nach Konzentration der Mutterlauge wurde der Rückstand durch zweimalige Säulenchromatographie auf Kieselgel $\left(\mathrm{CH}_{2} \mathrm{Cl}_{2} / \mathrm{Et}_{2} \mathrm{O}=6: 1\right.$; dann 
Pentan/EtOAc $=6: 1)$ gereinigt. Man erhielt den Aldehyd 96 (1.27 g, 3.67 mmol, 64\% über zwei Stufen) als weißen Feststoff.

$\mathbf{R}_{\mathbf{f}}=0.30\left(\mathrm{CH}_{2} \mathrm{Cl}_{2} / \mathrm{Et}_{2} \mathrm{O}=6: 1\right)$ oder $0.58(\operatorname{Petan} / \mathrm{EtOAc}=1: 1)$.

UV $\left(\mathrm{CH}_{3} \mathrm{CN}\right): \lambda_{\max }(\lg \varepsilon)=209.0 \mathrm{~nm}$ (4.769), 226.0 (4.548), 229.0 (4.546), 281.5 (3.536), 287.0 (3.533), 293.5 (3.563), 299.5 (3.568), 306.0 (3.709), 312.5 (3.647), 320.0 (3.858).

IR (KBr): $\widetilde{v}=2955 \mathrm{~cm}^{-1}(\mathrm{C}-\mathrm{H}), 2879,2841$ (Aldehyd C-H), $1713(\mathrm{C}=\mathrm{O}), 1405\left(\mathrm{CH}_{2}\right), 1099$, 1025 (C-O), 758, $695\left(\mathrm{CH}_{2}\right)$.

${ }^{1}$ H-NMR (600 MHz, $\mathrm{CDCl}_{3}$, Rotamer A/B = 0.38:0.62): $\delta=3.14\left(\mathrm{dd},{ }^{2} J=17.5 \mathrm{~Hz},{ }^{3} J=4.2\right.$ $\mathrm{Hz}, 0.38 \mathrm{H}, 2-\mathrm{H}_{\mathrm{a}}$, Rotamer A), 3.21 (dd, ${ }^{2} J=17.5 \mathrm{~Hz},{ }^{3} \mathrm{~J}=4.2 \mathrm{~Hz}, 0.62 \mathrm{H}, 2-\mathrm{H}_{\mathrm{a}}$, Rotamer B), $3.30\left(\mathrm{dd},{ }^{2} \mathrm{~J}=17.5 \mathrm{~Hz},{ }^{3} \mathrm{~J}=4.2 \mathrm{~Hz}, 0.38 \mathrm{H}, 2-\mathrm{H}_{\mathrm{b}}\right.$, Rotamer A), $3.54\left(\mathrm{dd},{ }^{2} \mathrm{~J}=17.5 \mathrm{~Hz},{ }^{3} \mathrm{~J}=4.2\right.$ $\mathrm{Hz}, 0.62 \mathrm{H}, 2-\mathrm{H}_{\mathrm{b}}$, Rotamer B), 4.82-5.04 (m, 2 H, 1'-H), 5.11-5.21 (m, $\left.1 \mathrm{H}, \mathrm{CH}_{\mathrm{a}} \mathrm{H}_{\mathrm{b}} \mathrm{Ph}\right)$, 5.21-5.31 (m, $\left.1 \mathrm{H}, \mathrm{CH}_{\mathrm{a}} \mathrm{H}_{\mathrm{b}} \mathrm{Ph}\right), 5.41-5.50$ (m, $1 \mathrm{H}, 3$ '-H), 7.30-7.44 (m, $\left.5 \mathrm{H}, 5 \times \mathrm{Ph}-\mathrm{H}\right)$, 7.48-7.57 (m, 1 H, 7'-H), 7.63-7.72 (m, 1 H, 6'-H), 7.75-7.84 (m, 1 H, 8'-H), 7.96 (s, 1 H, 9'-H), 7.97-8.06 (m, 1 H, 5’-H), 9.65 (s, 0.38 H, CHO, Rotamer A), 9.80 (s, 0.62 H, CHO, Rotamer B).

${ }^{13}$ C-NMR (150 MHz, $\mathrm{CDCl}_{3}$, Rotamer): $\delta=47.34,48.34$ (C-2), 49.98, 50.34 (C-1'), 58.40, 58.95 (C-3'), 67.20, 67.48 ( $\left.\mathrm{CH}_{2} \mathrm{Ph}\right), 126.52,126.57$ (C-7'), 127.45 (C-8'a), 127.73, 127.78 (C-8'), 127.93, 128.05, 128.13, 128.21, 128.28, 128.44, 128.49, 128.55 (C-9'a, p-Ph-C, 2 × o-Ph-C, $2 \times$ m-Ph-C), 128.82, 129.70 (C-5'), 129.39 (C-6'), 129.55 (C-9'), 135.94, 136.22 (ipso-Ph-C), 148.00 ( $\mathrm{NCO}_{2}$ ), 154.57, 154.74 (C-4'a), 160.73, 161.06 (C-3'a), 199.22, 199.52 (CHO).

MS (ESI): $m / z(\%)=347.1(100)[\mathrm{M}+\mathrm{H}]^{+}, 303.1(49)\left[\mathrm{M}-\mathrm{CH}_{2} \mathrm{CHO}\right]^{+}$.

$\mathrm{C}_{21} \mathrm{H}_{18} \mathbf{N}_{2} \mathbf{O}_{3}(346.38)$

Ber.: $\quad 347.1390 \quad[\mathrm{M}+\mathrm{H}]^{+}$

Gef.: $\quad 347.1390$ (ESI-HRMS) 


\section{8 (2-2,2,2-Trichlor-ethoxycarbonyl-2,3-dihydro-1H-pyrrolo[3,4- b]chinolin-3-yl)-acetaldehyd (114)}

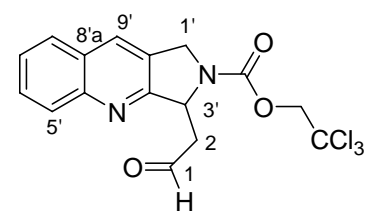

$\mathrm{Zu}$ einer Lösung von 100 (202 mg, $0.537 \mathrm{mmol}, 1.00$ Äq.) in $\mathrm{CH}_{2} \mathrm{Cl}_{2}(5.5 \mathrm{~mL})$ bei $-78^{\circ} \mathrm{C}$ wurde DIBAL-H (1.0 M in Dichlormethan, $0.600 \mathrm{~mL}, 1.12$ Äq.) zugetropft. Es wurde bei dieser Temperatur für 40 min gerührt. Die Reaktion wurde durch Zugabe von gesättiger Natrium-Kalium-Tartrat-Lösung $(4.5 \mathrm{~mL})$ beendet. Die Mischung wurde auf $0{ }^{\circ} \mathrm{C}$ erwärmt und mit $\mathrm{Et}_{2} \mathrm{O}(40 \mathrm{~mL})$ und Wasser $(10 \mathrm{~mL})$ versetzt. Die organische Phase wurden nachfolgend mit wäßriger NaOH-Lösung $(1 \mathrm{~N}, 2 \times 20 \mathrm{~mL})$, Wasser $(20 \mathrm{~mL})$ und gestättiger $\mathrm{NaCl}$-Lösung gewaschen, über $\mathrm{Na}_{2} \mathrm{SO}_{4}$ getrocknet, filtriert und im Vakuum konzentriert. Das erhaltene hellbraune Öl wurde mit $\mathrm{CH}_{3} \mathrm{CN}(10 \mathrm{~mL})$ und $\mathrm{K}_{2} \mathrm{CO}_{3}(15.0 \mathrm{mg}, 0.109$ mmol, 0.202 Äq.) versetzt. $\mathrm{Zu}$ dieser Mischung bei $-47^{\circ} \mathrm{C}$ wurde Troc-Cl (74.0 $\mu \mathrm{L}, 0.550 \mathrm{mmol}, 1.02$ Äq.) gegeben. Nach $1 \mathrm{~h}$ wurde auf $0{ }^{\circ} \mathrm{C}$ erwärmt und für $2 \mathrm{~h}$ gerührt. $\mathrm{K}_{2} \mathrm{CO}_{3}$ wurde abfiltriert. Nach Konzentration der Mutterlauge wurde der Rückstand durch Säulenchromatographie auf Kieselgel (Pentan/EtOAc = 3:1) gereinigt. Man erhielt den Aldehyd 114 (142 mg, 0.366 mmol, 68\% über zwei Stufen) als weißen Feststoff.

$\mathbf{R}_{\mathbf{f}}=0.31(\mathrm{Petan} / \mathrm{EtOAc}=3: 1)$.

UV $\left(\mathrm{CH}_{3} \mathrm{CN}\right): \lambda_{\max }(\lg \varepsilon)=208.0 \mathrm{~nm}$ (4.707), 225.0 (4.509), 281.5 (3.558), 287.0 (3.557), 293.0 (3.585), 299.5 (3.588), 306.0 (3.714), 312.5 (3.652), 319.5 (3.847).

IR $(\mathrm{KBr}): \widetilde{v}=2951 \mathrm{~cm}^{-1}(\mathrm{C}-\mathrm{H}), 1720(\mathrm{C}=\mathrm{O}), 1405\left(\mathrm{CH}_{2}\right), 1124,1059(\mathrm{C}-\mathrm{O}), 858,817$ (C-Cl), 755, $709\left(\mathrm{CH}_{2}\right)$.

${ }^{1} \mathbf{H}-\mathrm{NMR}\left(600 \mathrm{MHz}, \mathrm{CDCl}_{3}\right.$, Rotamer A/B =0.56:0.44): $\delta=3.26\left(\mathrm{dd},{ }^{2} \mathrm{~J}=17.9 \mathrm{~Hz},{ }^{3} \mathrm{~J}=4.7\right.$ Hz, 0.56 H, 2- $\mathrm{H}_{\mathrm{a}}$, Rotamer A), 3.39 (dd, ${ }^{2} J=17.9 \mathrm{~Hz},{ }^{3} J=3.5 \mathrm{~Hz}, 0.44 \mathrm{H}, 2-\mathrm{H}_{\mathrm{a}}$, Rotamer B), $3.58\left(\mathrm{dd},{ }^{2} J=17.9 \mathrm{~Hz},{ }^{3} J=4.7 \mathrm{~Hz}, 1 \mathrm{H}, 2-\mathrm{H}_{\mathrm{b}}\right), 4.75\left(\mathrm{~d},{ }^{2} J=12.0 \mathrm{~Hz}, 0.56 \mathrm{H}, \mathrm{OCH}_{\mathrm{a}} \mathrm{H}_{\mathrm{b}}\right.$, Rotamer A), $4.81\left(\mathrm{~d},{ }^{2} J=12.0 \mathrm{~Hz}, 0.44 \mathrm{H}, \mathrm{OCH}_{\mathrm{a}} \mathrm{H}_{\mathrm{b}}\right.$, Rotamer B), 4.86-4.97 (m, $1.44 \mathrm{H}$, $\left.\mathrm{OCH}_{\mathrm{a}} \mathrm{H}_{\mathrm{b}}, 1^{\prime}-\mathrm{H}_{\mathrm{a}}\right), 4.98-5.08\left(\mathrm{~m}, 1.56 \mathrm{H}, 1^{\prime}-\mathrm{H}_{\mathrm{a}}, 1^{\prime}-\mathrm{H}_{\mathrm{b}}\right), 5.45\left(\mathrm{~m}_{\mathrm{c}}, 0.56 \mathrm{H}, 3\right.$ '-H, Rotamer A), 5.49 ( $\mathrm{m}_{\mathrm{c}}, 0.44$ H, 3'-H, Rotamer B), 7.49-7.57 (m, 1 H, 7'-H), 7.65-7.72 (m, 1 H, 6'-H), 7.81 
(d, $\left.{ }^{3} J=8.2 \mathrm{~Hz}, 1 \mathrm{H}, 8^{\prime}-\mathrm{H}\right), 7.98-8.06$ (m, 2 H, 5'-H, 9'-H), 9.74 (s, 0.44 H, CHO, Rotamer B), 9.77 (s, 0.56 H, CHO, Rotamer A).

${ }^{13}$ C-NMR (125 MHz, $\mathrm{CDCl}_{3}$, Rotamer): $\delta=46.91,47.71$ (C-2), 50.23, 50.85 (C-1'), 58.66, 59.15 (C-3'), 74.95, $75.04\left(\mathrm{OCH}_{2}\right), 95.30,95.44\left(\mathrm{CCl}_{3}\right), 126.71(\mathrm{C}-7$ '), $127.56(\mathrm{C}-8$ 'a), 127.84, 127.88 (C-8'), 127.91, 128.10 (C-9'a), 128.91, 128.95 (C-9'), 129.54, 129.59 (C-6’), 129.73, 129.75 (C-5'), 148.14, $148.17\left(\mathrm{NCO}_{2}\right), 152.79,152.97$ (C-4'a), 160.33, 160.80 (C-3’a), 198.88, 199.09 (CHO).

MS (ESI): $m / z(\%)=389.0(40), 387.0(42)[\mathrm{M}+\mathrm{H}]^{+}, 344.9(96), 342.9(100)\left[\mathrm{M}-\mathrm{CH}_{2} \mathrm{CHO}\right]^{+}$.

$\mathbf{C}_{16} \mathbf{H}_{13} \mathbf{C l}_{3} \mathbf{N}_{2} \mathbf{O}_{3}(387.64) \quad$ Ber.: $\quad 387.0064,389.0035[\mathrm{M}+\mathrm{H}]^{+}$ $342.9802,344.9772 \quad\left[\mathrm{M}-\mathrm{CH}_{2} \mathrm{CHO}\right]^{+}$

Gef.: $\quad 387.0065,389.0035$ (ESI-HRMS)

$342.9803,344.9773$

\section{9 (2-(4-Methoxybenzyl)-2,3-dihydro-1H-pyrrolo[3,4-b]chinolin-3-yl)- acetaldehyd (71)}

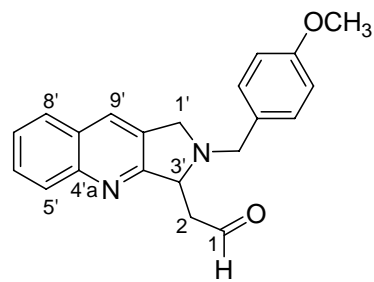

$\mathrm{Zu}$ einer Lösung von 100 (111 mg , $0.295 \mathrm{mmol}, 1.00$ Äq.) in $\mathrm{CH}_{2} \mathrm{Cl}_{2}$ (3 mL) bei $-78^{\circ} \mathrm{C}$ wurde DIBAL-H (1.0 M in Dichlormethan, $0.300 \mathrm{~mL}, 1.02$ Äq.) zugetropft. Nach $25 \mathrm{~min}$ Rühren bei dieser Temperatur wurde DIBAL-H (1.0 M in Dichlormethan, $0.150 \mathrm{~mL}, 0.508$ Äq.) weiter gegeben. Nach 30 min Rühren wurde die Reaktion durch Zugabe von Methanol $(1.0 \mathrm{~mL})$ beendet. Die Mischung wurde dann auf $0{ }^{\circ} \mathrm{C}$ erwärmt und mit gesättiger NatriumKalium-Tartrat-Lösung $(5 \mathrm{~mL})$ und Wasser $(5 \mathrm{~mL})$ versetzt. Die wäßrige Phase wurden mit $\mathrm{CH}_{2} \mathrm{Cl}_{2}(2 \times 10 \mathrm{~mL})$ extrahiert. Die vereinigten organischen Phasen wurden über $\mathrm{Na}_{2} \mathrm{SO}_{4}$ getrocknet, filtriert und im Vakuum konzentriert. Man erhielt das Rohprodukt des Aldehyds 71 (99 mg, 0.298 mmol, 101\%) als braunes Öl. Ein Teil (22 mg) des Rohproduktes wurde in $\mathrm{Et}_{2} \mathrm{O}(5 \mathrm{~mL})$ aufgenommen und mit wässriger gesättiger $\mathrm{NaHSO}_{3}$-Lösung $(3 \times 5 \mathrm{~mL})$ 
extrahiert. Die vereinigten wässrigen Phasen wurden auf $0{ }^{\circ} \mathrm{C}$ gekühlt und mit Natronlauge ( $5 \mathrm{~N}, 17 \mathrm{~mL}$ ) versetzt, wobei der $\mathrm{pH}$-wert bei 13 lag. Die wässrige Phase wurde dann mit $\mathrm{Et}_{2} \mathrm{O}(2 \times 20 \mathrm{~mL})$ extrahiert. Die vereinigten organischen Phasen wurden dann mit gesättiger $\mathrm{NaCl}$-Lösung gewaschen, über $\mathrm{Na}_{2} \mathrm{SO}_{4}$ getrocknet und filtriert. Nach Entfernung des Lösungsmittels erhielt man den Aldehyd 71 (12 mg, 0.036 mmol, 55\%) als braunes Öl.

$\mathbf{R}_{\mathbf{f}}=0.43($ Petan $/$ EtOAc $=2: 1$, Zersetzung auf DC-Kiesegel $)$.

UV ( $\left.\mathrm{CH}_{3} \mathrm{CN}\right): \lambda_{\max }(\lg \varepsilon)=195.5 \mathrm{~nm}$ (4.554), 209.0 (4.511), 230.5 (4.447), 273.5 (3.640), 306.5 (3.477), 319.5 (3.514).

IR (KBr): $\widetilde{v}=2962 \mathrm{~cm}^{-1}(\mathrm{C}-\mathrm{H}), 2834\left(\right.$ Aldehyd C-H), $1721(\mathrm{C}=\mathrm{O}), 1511\left(\mathrm{CH}_{3}\right), 1252\left(\mathrm{CH}_{2}\right)$, $1033(\mathrm{C}-\mathrm{O}), 818\left(\mathrm{CH}_{2}\right)$.

${ }^{1} \mathbf{H}-\mathbf{N M R}\left(600 \mathrm{MHz}, \mathrm{CDCl}_{3}\right): \delta=3.11\left(\mathrm{ddd},{ }^{2} \mathrm{~J}=16.6 \mathrm{~Hz},{ }^{3} \mathrm{~J}=5.4 \mathrm{~Hz},{ }^{3} \mathrm{~J}=3.9 \mathrm{~Hz}, 1 \mathrm{H}\right.$, 2- $\left.\mathrm{H}_{\mathrm{a}}\right), 3.27\left(\mathrm{ddd},{ }^{2} J=16.6 \mathrm{~Hz},{ }^{3} J=3.9 \mathrm{~Hz},{ }^{3} J=1.1 \mathrm{~Hz}, 1 \mathrm{H}, 2-\mathrm{H}_{\mathrm{b}}\right), 3.59\left(\mathrm{~d},{ }^{2} J=12.9 \mathrm{~Hz}, 1 \mathrm{H}\right.$, $\left.\mathrm{CH}_{\mathrm{a}} \mathrm{H}_{\mathrm{b}} \mathrm{Ph}(\mathrm{p}-\mathrm{OMe})\right), 3.70\left(\mathrm{~d},{ }^{2} \mathrm{~J}=13.7 \mathrm{~Hz}, 1 \mathrm{H}, 1{ }^{\prime}-\mathrm{H}_{\mathrm{a}}\right), 3.80\left(\mathrm{~s}, 3 \mathrm{H}, \mathrm{OCH}_{3}\right), 4.16\left(\mathrm{~d},{ }^{2} \mathrm{~J}=13.7\right.$ $\mathrm{Hz}, 1 \mathrm{H}, 1$ '- $\left.\mathrm{H}_{\mathrm{b}}\right), 4.19\left(\mathrm{~d},{ }^{2} J=12.9 \mathrm{~Hz}, 1 \mathrm{H}, \mathrm{CH}_{\mathrm{a}} H_{\mathrm{b}} \mathrm{Ph}(p-\mathrm{OMe})\right), 4.42\left(\mathrm{~m}_{\mathrm{c}}, 1 \mathrm{H}, 3^{\prime}-\mathrm{H}\right)$, 6.85-6.89 (m, 2 H, $2 \times m-\mathrm{Ph}(p-\mathrm{OMe})-\mathrm{H}), 7.24-7.27$ (m, $2 \mathrm{H}, 2 \times o-\mathrm{Ph}(p-\mathrm{OMe})-\mathrm{H}), 7.48\left(\mathrm{~m}_{\mathrm{c}}\right.$, $\left.1 \mathrm{H}, 7^{\prime}-\mathrm{H}\right), 7.65$ (m $\left.\mathrm{c}, 1 \mathrm{H}, 6^{\prime}-\mathrm{H}\right), 7.74$ (d, $\left.{ }^{3} \mathrm{~J}=8.2 \mathrm{~Hz}, 1 \mathrm{H}, 8^{\prime}-\mathrm{H}\right), 7.83$ (s, $\left.1 \mathrm{H}, 9^{\prime}-\mathrm{H}\right), 8.04$ (d, $\left.{ }^{3} J=8.6 \mathrm{~Hz}, 1 \mathrm{H}, 5^{\prime}-\mathrm{H}\right), 9.70\left(\mathrm{dd},{ }^{3} J=3.9 \mathrm{~Hz},{ }^{3} \mathrm{~J}=1.1 \mathrm{~Hz}, 1 \mathrm{H}, \mathrm{CHO}\right)$.

${ }^{13}$ C-NMR (125 MHz, $\left.\mathrm{CDCl}_{3}\right): \delta=45.43(\mathrm{C}-2), 55.24\left(\mathrm{OCH}_{3}\right), 55.41\left(\mathrm{C}-1\right.$ ') $57.29\left(\mathrm{CH}_{2} \mathrm{Ph}\right)$, 63.76 (C-3’), 113.8 (2 × m-Ph(p-OMe)-C), (126.2 (C-7'), 127.7 (C-9'a), 127.7 (C-8'), 128.9 (C-5’), 128.9 (C-9’), 129.0 (C-6’), 130.0 (2 × o-Ph(p-OMe)-C), 130.1 (ipso-Ph(p-OMe)-C), 131.2 (C-8'a), 147.9 (C-4'a), 158.9 (p-Ph(p-OMe)-C), 163.8 (C-3'a), 201.8 (CHO).

MS (ESI): $m / z(\%)=333.2(35)[\mathrm{M}+\mathrm{H}]^{+}, 289.1(54)\left[\mathrm{M}-\mathrm{CH}_{2} \mathrm{CHO}^{+}\right.$.

$\mathbf{C}_{21} \mathbf{H}_{20} \mathbf{N}_{2} \mathbf{O}_{2}(332.40)$

Ber.: $\quad 333.1597$

Gef: $\quad 333.1598$

$[\mathrm{M}+\mathrm{H}]^{+}$

(ESI-HRMS) 


\subsection{0 (2-1-Chlor-ethoxycarbonyl-2,3-dihydro-1H-pyrrolo[3,4-b]chinolin-3-} yl)-acetaldehyd (115)

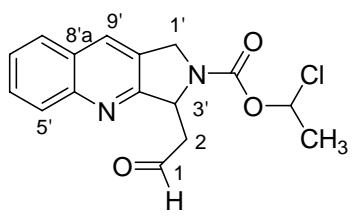

Das Rohprodukt (siehe 3.9 bei der Synthese von 71) des Aldehyds 71 (77 mg, 0.23 mmol, 1.0 Äq.) wurde ohne weitere Reinigung in $\mathrm{CH}_{3} \mathrm{CN}$ gelöst und auf $-10^{\circ} \mathrm{C}$ gekühlt. $\mathrm{ACE}-\mathrm{Cl}$ (28 $\mu \mathrm{L}, 0.26 \mathrm{mmol}, 1.1$ Äq.) wurde gegeben. Nach $1 \mathrm{~h}$ Rühren bei dieser Temperatur wurde ACE-Cl (35 $\mu \mathrm{L}, 0.32$ mmol, 1.4 Äq.) weiter gegeben. Die Reaktionsmischung wurde dann bei $15^{\circ} \mathrm{C}$ für $3 \mathrm{~h}$ gerührt. Nach Entfernung des Lösungsmittels wurde der Rückstand durch Säulenchromatographie auf Kieselgel $($ Pentan/EtOAc $=2: 1)$ gereinigt. Man erhielt den Aldehyd 115 (40 mg, 0.12 mmol, 54\% über zwei Stufen) als hellgelbes Öl.

$\mathbf{R}_{\mathbf{f}}=0.36(\operatorname{Petan} / \operatorname{EtOAc}=2: 1)$.

UV $\left(\mathrm{CH}_{3} \mathrm{CN}\right): \lambda_{\max }(\lg \varepsilon)=208.0 \mathrm{~nm}$ (4.681), 225.0 (4.485), 281.0 (3.577), 293.0 (3.585), 299.5 (3.584), 306.0 (3.701), 312.5 (3.642), 319.5 (3.827).

IR (KBr): $\widetilde{v}=2925 \mathrm{~cm}^{-1}(\mathrm{C}-\mathrm{H}), 1729(\mathrm{C}=\mathrm{O}), 1403\left(\mathrm{CH}_{2}\right), 1325\left(\mathrm{CH}_{3}\right), 1131,1097,1030$ (C-O), 901, $861(\mathrm{C}-\mathrm{Cl}), 757,735\left(\mathrm{CH}_{2}, \mathrm{CH}_{3}\right)$.

${ }^{1} \mathbf{H}-\mathbf{N M R}\left(300 \mathrm{MHz}, \mathrm{CDCl}_{3}\right.$, Rotamer A/B = 4:1): $\delta=1.84-1.94\left(\mathrm{~m}, 3 \mathrm{H}, \mathrm{CH}_{3}\right), 3.11-3.82$ (m, 2 H, 2-H), 4.80-5.09 (m, 2 H, 1'-H), 5.36-5.54 (m, 1 H, 3'-H), 6.56-6.77 (m, $1 \mathrm{H}$, OCHCl), 7.46-7.61 (m, $\left.1 \mathrm{H}, 7^{\prime}-\mathrm{H}\right), 7.63-7.76\left(\mathrm{~m}, 1 \mathrm{H}, 6^{\prime}-\mathrm{H}\right), 7.82\left(\mathrm{~d},{ }^{3} J=8.2 \mathrm{~Hz}, 1 \mathrm{H}\right.$, 8'-H), 7.95-8.08 (m, 2 H, 5'-H, 9'-H), 9.78 (s, 0.80 H, CHO, Rotamer A), 9.80 (s, 0.20 H, CHO, Rotamer B).

MS (ESI): $m / z(\%)=319.1(74)[\mathrm{M}+\mathrm{H}]^{+}, 275.1(100)\left[\mathrm{M}-\mathrm{CH}_{2} \mathrm{CHO}\right]^{+}$.

$\mathrm{C}_{16} \mathrm{H}_{15} \mathrm{ClN}_{2} \mathrm{O}_{3}(318.75)$

Ber.: $\quad 319.0844 \quad[\mathrm{M}+\mathrm{H}]^{+}$

Gef.: $\quad 319.0844 \quad$ (ESI-HRMS) 


\subsection{2-(2-Benzyl-2,3-dihydro-1H-pyrrolo[3,4-b]chinolin-3-yl)-Ethanol (95)}

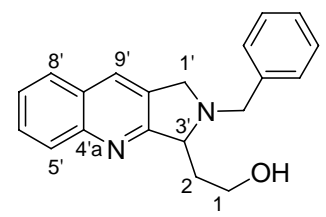

$\mathrm{Zu}$ einer Mischung von $\mathrm{LiAlH}_{4}\left(5.4 \mathrm{mg}, 0.14 \mathrm{mmol}, 1.0\right.$ Äq.) in THF (2 mL) bei $-78{ }^{\circ} \mathrm{C}$ wurde eine Lösung von 93 (49 mg, 0.14 mmol, 1.0 Äq.) in THF (2 mL) zugetropft. Es wurde für $1 \mathrm{~h}$ bei dieser Temperatur gerührt und dann auf RT erwärmt. Nach $24 \mathrm{~h}$ wurde $\mathrm{LiAlH}_{4}$ (26 mg, 0.70 mmol, 5.0 Äq.) hinzufügt und die Reaktionsmischung für $3 \mathrm{~h}$ bei RT gerührt. Bei Abkühlung auf $0{ }^{\circ} \mathrm{C}$ wurde vorsichtig Wasser $(5 \mathrm{~mL})$ zugegeben dann wieder auf RT erwärmt. Die wässrige Phase wurde mit $\mathrm{CH}_{2} \mathrm{Cl}_{2}(2 \times 10 \mathrm{~mL})$ extrahiert. Die vereinigten organischen Phasen wurden filtriert, über $\mathrm{MgSO}_{4}$ getrocknet und im Vakuum konzentriert. Der Rückstand wurde in $\mathrm{Et}_{2} \mathrm{O}$ aufgenommen. Nach Filtration wurde das Lösungsmittel entfernt. Man erhielt 95 (25 mg, 0.082 mmol, 59\%) als braunes Öl.

$\mathbf{R}_{\mathbf{f}}=0.27($ Petan $/$ EtOAc $=2: 1)$.

${ }^{1} \mathbf{H}-\mathbf{N M R}\left(300 \mathrm{MHz}, \mathrm{CDCl}_{3}\right): \delta=2.23-2.45(\mathrm{~m}, 2 \mathrm{H}, 2-\mathrm{H}), 3.60\left(\mathrm{~d},{ }^{2} J=12.8 \mathrm{~Hz}, \mathrm{CH}_{\mathrm{a}} \mathrm{H}_{\mathrm{b}} \mathrm{Ph}\right)$, $3.71\left(\mathrm{~d},{ }^{2} \mathrm{~J}=14.0 \mathrm{~Hz}, 1 \mathrm{H}, 1^{\prime}-\mathrm{H}_{\mathrm{a}}\right), 3.86-3.96\left(\mathrm{~m}, 2 \mathrm{H}, \mathrm{CH}_{2} \mathrm{OH}\right), 4.14-4.25$ (m, $2 \mathrm{H}, 3^{\prime}-\mathrm{H}$, $\left.1^{\prime}-\mathrm{H}_{\mathrm{b}}\right), 4.41\left(\mathrm{~d},{ }^{2} \mathrm{~J}=12.8 \mathrm{~Hz}, 1 \mathrm{H}, \mathrm{CH}_{\mathrm{a}} \mathrm{H}_{\mathrm{b}} \mathrm{Ph}\right), 6.33\left(\mathrm{~s}_{\mathrm{br}}, 1 \mathrm{H}, \mathrm{OH}\right), 7.27-7.45(\mathrm{~m}, 5 \mathrm{H}, 5 \times$ Ph-H), $7.50\left(\mathrm{~m}_{\mathrm{c}}, 1 \mathrm{H}, 7^{\prime}-\mathrm{H}\right), 7.66\left(\mathrm{~m}_{\mathrm{c}}, 1 \mathrm{H}, 6^{\prime}-\mathrm{H}\right), 7.76$ (d, $\left.{ }^{3} \mathrm{~J}=8.1 \mathrm{~Hz}, 1 \mathrm{H}, 8^{\prime}-\mathrm{H}\right), 7.85$ (s, $\left.1 \mathrm{H}, 9^{\prime}-\mathrm{H}\right), 8.05$ (d, $\left.{ }^{3} \mathrm{~J}=8.4 \mathrm{~Hz}, 1 \mathrm{H}, 5^{\prime}-\mathrm{H}\right)$.

${ }^{13}$ C-NMR (75 MHz, $\left.\mathrm{CDCl}_{3}\right): \delta=33.02(\mathrm{C}-2), 55.62\left(\mathrm{C}-1{ }^{\prime}\right), 58.55\left(\mathrm{CH}_{2} \mathrm{Ph}\right), 61.09\left(\mathrm{CH}_{2} \mathrm{OH}\right)$, 69.82 (C-3'), 126.4 (C-7'), 127.5 (p-Ph-C), 127.6 (C-9' ' $\left.{ }^{*}\right), 127.7\left(\mathrm{C}-8^{*}{ }^{*}\right), 128.6$ (C-5', $2 \times m-$ Ph-C), 129.0 (2 × o-Ph-C), 129.1 (C-6’), 129.4 (C-9'), 131.2 (C-8'a), 137.8 (ipso-Ph-C), 147.1 (C-4'a), 164.4 (C-3'a).

MS (70 eV, EI): $m / z(\%)=304.2(2)[\mathrm{M}]^{+}, 259.2(100)\left[\mathrm{M}-\mathrm{CH}_{2} \mathrm{CH}_{2} \mathrm{OH}\right]^{+}, 91.1(72)\left[\mathrm{C}_{7} \mathrm{H}_{7}\right]^{+}$. $\mathbf{C}_{20} \mathbf{H}_{20} \mathbf{N}_{2} \mathbf{O}$ (304.39). 


\subsection{2 (2-2,2,2-Trichlor-ethoxycarbonyl-2,3-dihydro-1H-pyrrolo[3,4- b]chinolin-3-yl)-Essigsäureethylester (110)}

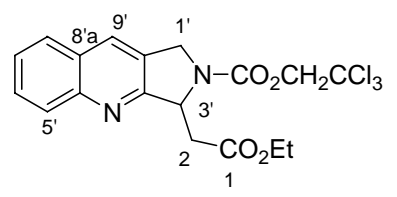

$\mathrm{Zu}$ einer Lösung von 93 (150 mg, $0.433 \mathrm{mmol}, 1.00$ Äq.) in $\mathrm{CH}_{3} \mathrm{CN}$ (3 mL) bei RT wurde Troc-Cl (58.0 $\mu \mathrm{L}, 0.433 \mathrm{mmol}, 1.00$ Äq.) gegeben. Es wurde für $16 \mathrm{~h}$ gerührt. Nach Entfernung des Lösungsmittels wurde der Rückstand durch Säulenchromatographie auf Kieselgel (Pentan/EtOAc = 5:1) gereinigt. Man erhielt den Ester 110 (138 mg, 0.320 mmol, $74 \%$ ) als braunes Öl.

$\mathbf{R}_{\mathbf{f}}=0.80(\mathrm{Petan} / \mathrm{EtOAc} / \mathrm{MeOH}=1: 1: 0.2)$.

${ }^{1}$ H-NMR (300 MHz, $\mathrm{CDCl}_{3}$, Rotamer A/B = 0.5:0.5): $\delta=1.09\left(\mathrm{~m}_{\mathrm{c}}, 3 \mathrm{H}, \mathrm{CH}_{3}\right), 3.29\left(\mathrm{dd},{ }^{2} \mathrm{~J}=\right.$ $16.8 \mathrm{~Hz},{ }^{3} \mathrm{~J}=3.5 \mathrm{~Hz}, 0.5 \mathrm{H}, 2-\mathrm{H}_{\mathrm{a}}$, Rotamer A), $3.39\left(\mathrm{dd},{ }^{2} \mathrm{~J}=16.8 \mathrm{~Hz},{ }^{3} \mathrm{~J}=2.6 \mathrm{~Hz}, 0.5 \mathrm{H}\right.$, 2- $\mathrm{H}_{\mathrm{a}}$, Rotamer B), 3.47-3.62 (m, $1 \mathrm{H}, 2-\mathrm{H}_{\mathrm{b}}$, Rotamer A und B), 3.84-4.03 (m, $\left.2 \mathrm{H}, \mathrm{CH}_{2} \mathrm{CH}_{3}\right)$, 4.74-5.12 (m, 4 H, $\left.\mathrm{CH}_{2} \mathrm{CCl}_{3}, 1^{\prime}-\mathrm{H}\right), 5.35-5.48$ (m, $\left.1 \mathrm{H}, 3^{\prime}-\mathrm{H}\right), 7.54\left(\mathrm{~m}_{\mathrm{c}}, 1 \mathrm{H}, 7^{\prime}-\mathrm{H}\right), 7.70\left(\mathrm{~m}_{\mathrm{c}}\right.$, $\left.1 \mathrm{H}, 6^{\prime}-\mathrm{H}\right), 7.83$ (d, $\left.{ }^{3} \mathrm{~J}=8.1 \mathrm{~Hz}, 1 \mathrm{H}, 8^{\prime}-\mathrm{H}\right), 7.98-8.11$ (m, 2 H, 5'-H, 9'-H).

MS (70 eV, EI): $m / z \quad(\%)=432.0$ (81), 430.0 (84) $[\mathrm{M}]^{+}, 345.0$ (96), 343.0 (100) $\left[\mathrm{M}-\mathrm{CH}_{2} \mathrm{CO}_{2} \mathrm{Et}\right]^{+}, 169.1(78)\left[\mathrm{M}-\mathrm{CH}_{2} \mathrm{CO}_{2} \mathrm{Et}-\mathrm{CO}_{2} \mathrm{CH}_{2} \mathrm{CCl}_{3}\right]^{+}$.

$\mathrm{C}_{18} \mathrm{H}_{17} \mathrm{Cl}_{3} \mathbf{N}_{2} \mathbf{O}_{4}$ (431.69). 


\subsection{3 (2-Benzyloxycarbonyl-2,3-dihydro-1H-pyrrolo[3,4-b]chinolin-3-yl)-}

\section{Essigsäureethylester (97)}

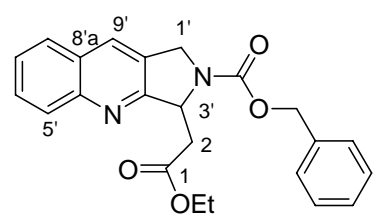

Zu einer Lösung von 93 (398 mg, $1.15 \mathrm{mmol}, 1.00$ Äq.) in $\mathrm{CH}_{3} \mathrm{CN}$ (8 mL) bei $0{ }^{\circ} \mathrm{C}$ wurde Cbz-Cl (0.270 mL, 1.90 mmol, 1.65 Äq.) gegeben. Nach 4.5 h Rühren bei dieser Temperatur wurde Cbz-Cl (0.220 mL, $1.50 \mathrm{mmol}, 1.35$ Äq.) weiter gegeben und die Reaktionsmischung auf RT erwärmt. Nach $16 \mathrm{~h}$ wurde die Reaktion durch Zugabe von gesättiger $\mathrm{NaHCO}_{3^{-}}$ Lösung $(10 \mathrm{~mL})$ und Wasser $(5 \mathrm{~mL})$ beendet. Die wässrige Phase wurde mit EtOAc $(2 \times 20$ $\mathrm{mL}$ ) extrahiert. Die vereinigten organischen Phasen wurden mit gesättiger NaCl-Lösung gewaschen, über $\mathrm{MgSO}_{4}$ getrocknet und filtriert. Nach Entfernung des Lösungsmittels wurde der Rückstand durch Säulenchromatographie auf Kieselgel (Pentan/EtOAc $=4: 1)$ gereinigt. Man erhielt den Ester 97 (349 mg, 0.893 mmol, 78\%) als weißen Feststoff.

$\mathbf{R}_{\mathbf{f}}=0.18($ Pentan $/$ EtOAc $=4: 1)$.

UV $\left(\mathrm{CH}_{3} \mathrm{CN}\right): \lambda_{\max }(\lg \varepsilon)=208.5 \mathrm{~nm}$ (4.805), 226.0 (4.582), 229.0 (4.583), 305.5 (3.742), 312.0 (3.675), 319.5 (3.893).

IR (KBr): $\widetilde{v}=2980 \mathrm{~cm}^{-1}(\mathrm{C}-\mathrm{H}), 1706(\mathrm{C}=\mathrm{O}), 1405,1356,1325\left(\mathrm{CH}_{2}\right), 1112,1016(\mathrm{C}-\mathrm{O})$, 755, $698\left(\mathrm{CH}_{2}\right)$.

${ }^{1} \mathbf{H}-\mathbf{N M R}\left(300 \mathrm{MHz}, \mathrm{CDCl}_{3}\right.$, Rotamer A/B $\left.=0.5: 0.5\right): \delta=1.01-1.12\left(\mathrm{~m}, 3 \mathrm{H}, \mathrm{CH}_{3}\right)$, 3.17-3.36 (m, 1.5 H, 2- $\mathrm{H}_{\mathrm{a}}$, Rotamer A und 2-H, Rotamer B), $3.55\left(\mathrm{dd},{ }^{2} J=16.8 \mathrm{~Hz},{ }^{3} J=4.9\right.$ $\mathrm{Hz}, 0.5 \mathrm{H}, 2-\mathrm{H}_{\mathrm{b}}$, Rotamer A), 3.84-4.02 (m, $\left.2 \mathrm{H}, \mathrm{CH}_{2} \mathrm{CH}_{3}\right), 4.80-5.06$ (m, $2 \mathrm{H}, 1$ '- $\left.\mathrm{H}\right)$, 5.15-5.43 (m, 3 H, 3'-H, CH $\left.\mathrm{CH}_{2} \mathrm{Ph}\right), 7.29-7.47$ (m, $\left.5 \mathrm{H}, 5 \times \mathrm{Ph}-\mathrm{H}\right), 7.51$ (m $\left., 1 \mathrm{H}, 7^{\prime}-\mathrm{H}\right), 7.67$ (m $\left.\mathrm{m}_{\mathrm{c}}, 1 \mathrm{H}, 6^{\prime}-\mathrm{H}\right), 7.75-7.85$ (m, 1 H, 8'-H), 7.90-8.10 (m, 2 H, 5'-H, 9'-H).

MS (ESI): $m / z(\%)=391.1(100)[\mathrm{M}+\mathrm{H}]^{+}$.

$\mathbf{C}_{23} \mathbf{H}_{22} \mathbf{N}_{2} \mathbf{O}_{4}(390.43)$

Ber.: $\quad 391.1652$

$[\mathrm{M}+\mathrm{H}]^{+}$

413.1471

$[\mathrm{M}+\mathrm{Na}]^{+}$

429.1211

$[\mathrm{M}+\mathrm{K}]^{+}$

Gef.: $\quad 391.1650$

(ESI-HRMS)

413.1470

429.1208 


\subsection{4 (2,3-dihydro-1H-pyrrolo[3,4-b]chinolin-3-yl)-Essigsäureethylester}

(98)

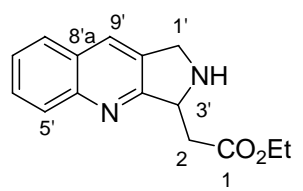

\section{Ausgehend von 110 durch N-Troc-Entschützung}

Zu einer Lösung von 110 (790 mg, 1.83 mmol, 1.00 Äq.) in Essigsäure (15 mL) bei RT wurde Zink (Pulver, $299 \mathrm{mg}, 4.57 \mathrm{mmol}, 2.50$ Äq.) gegeben. Nach $45 \mathrm{~h}$ wurde Zink-Pulver abfiltriert und mit Ethanol gewaschen. Die Mutterlauge wurde im Vakuum konzentriert und der Rückstand in EtOAc (100 mL), gesättiger $\mathrm{Na}_{2} \mathrm{CO}_{3}$-Lösung $(15 \mathrm{~mL})$ und Wasser $(10 \mathrm{~mL})$ aufgenommen. Nach Phasentrennung wurde die organische Phase mit Wasser und gesättiger$\mathrm{NaCl}$-Lösung gewaschen, über $\mathrm{MgSO}_{4}$ getrocknet, filtriert und im Vakuum konzentriert. Der Rückstand wurde durch Säulenchromatographie auf Kieselgel (Pentan/EtOAc/MeOH = 1:1:0.4) gereinigt. Man erhielt das Amin 98 (212 mg, 0.827 mmol, 45\%) als braunes Öl.

\section{Ausgehend von 97 durch N-Cbz-Entschützung}

Eine Mischung von 97 (17 mg, 0.044 mmol, 1.0 Äq.) und Pd/C (10\% Pd, 5 mg) in EtOH (0.9 $\mathrm{mL}$ ) wurde bei $\mathrm{RT}$ unter $\mathrm{H}_{2}$-Atmosphäre für $1 \mathrm{~h}$ gerührt. $\mathrm{Pd} / \mathrm{C}$ wurde abfiltriert und die Konzentration der Mutterlauge lieferte das Amin 98 (11 mg. 0.044 mmol, quant.) als hellbraunes Öl.

$\mathbf{R}_{\mathbf{f}}=0.28($ Petan $/$ EtOAc $/ \mathrm{MeOH}=1: 1: 0.2)$.

${ }^{1}$ H-NMR $\left(300 \mathrm{MHz}, \mathrm{CDCl}_{3}\right): \delta=1.27\left(\mathrm{t},{ }^{3} \mathrm{~J}=7.1 \mathrm{~Hz}, 3 \mathrm{H}, \mathrm{CH}_{3}\right), 2.48\left(\mathrm{~s}_{\mathrm{br}}, 1 \mathrm{H}, \mathrm{NH}\right), 2.69$ $\left(\mathrm{dd},{ }^{2} J=16.6 \mathrm{~Hz},{ }^{3} J=9.8 \mathrm{~Hz}, 1 \mathrm{H}, 2-\mathrm{H}_{\mathrm{a}}\right), 3.32\left(\mathrm{dd},{ }^{2} J=16.6 \mathrm{~Hz},{ }^{3} J=2.9 \mathrm{~Hz}, 1 \mathrm{H}, 2-\mathrm{H}_{\mathrm{b}}\right), 4.20$ (q, ${ }^{3} J=7.1 \mathrm{~Hz}, 2 \mathrm{H}, \mathrm{CH}_{2} \mathrm{CH}_{3}$ ), 4.42 (s, $2 \mathrm{H}, 1$ '-H), 4.89 (dd, ${ }^{3} J=2.9,9.8 \mathrm{~Hz}, 1 \mathrm{H}, 3$ '-H), 7.51 $\left(\mathrm{m}_{\mathrm{c}}, 1 \mathrm{H}, 7^{\prime}-\mathrm{H}\right), 7.67$ ( $\left.\mathrm{m}_{\mathrm{c}}, 1 \mathrm{H}, 6^{\prime}-\mathrm{H}\right), 7.79$ (d, $\left.{ }^{3} \mathrm{~J}=8.2 \mathrm{~Hz}, 1 \mathrm{H}, 8^{\prime}-\mathrm{H}\right), 7.96$ (s, $\left.1 \mathrm{H}, 9^{\prime}-\mathrm{H}\right)$, $8.05\left(\mathrm{~d},{ }^{3} \mathrm{~J}=8.5 \mathrm{~Hz}, 1 \mathrm{H}, 5^{\prime}-\mathrm{H}\right)$.

${ }^{13}$ C-NMR (125 MHz, $\left.\mathrm{CDCl}_{3}\right): \delta=14.20\left(\mathrm{CH}_{3}\right), 39.90$ (C-2), 48.97 (C-1'), 59.30 (C-3'), $60.55\left(\mathrm{CH}_{2} \mathrm{CH}_{3}\right), 126.1$ (C-7'), 127.5 (C-9'a*), $127.7\left(\mathrm{C}-8^{* *}\right), 128.9$ (C-5', C-6'), 129.2 (C-9'), 132.4 (C-8'a), 147.9 (C-4'a), 164.9 (C-3'a), $172.3\left(\mathrm{CO}_{2} \mathrm{Et}\right)$.

MS (70 eV, EI): $m / z(\%)=256.1(33)[\mathrm{M}]^{+}, \quad 183.1 \quad(23) \quad\left[\mathrm{M}-\mathrm{CO}_{2} \mathrm{Et}\right]^{+} 169.1$ (100) $\left[\mathrm{M}-\mathrm{CH}_{2} \mathrm{CO}_{2} \mathrm{Et}\right]^{+}$.

$\mathbf{C}_{15} \mathbf{H}_{16} \mathbf{N}_{2} \mathbf{O}_{2}(256.30)$ 
4 Durchführung der Domino-Knoevenagel-hetero-Diels-AlderReaktion und anschließende Bildung des Lactams

\subsection{4-(2-Benzyloxycarbonyl-2,3-dihydro-1H-pyrrolo[3,4-b]chinolin-3- ylmethyl)-6-ethoxy-5-ethyl-tetrahydropyran-2-on (117)}

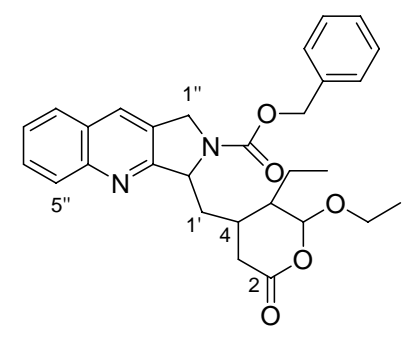

Eine Mischung von 96 (769 mg, 2.22 mmol, 1.00 Äq.), Meldrum-Säure (320 mg, 2.22 mmol, 1.00 Äq.), EDDA (16.0 mg, $89.0 \mu \mathrm{mol}, 4.00$ mol\% ) und 64 (889 mg, 8.88 mmol, 4.00 Äq.) in Toluol $(22 \mathrm{~mL})$ wurde bei $50{ }^{\circ} \mathrm{C}$ für $19 \mathrm{~h}$ erhitzt. Nach Abkühlung auf RT wurde die Reaktionsmischung über $\mathrm{Na}_{2} \mathrm{SO}_{4}$ getrocknet, filtriert. Nach Entfernung des Lösungsmittels wurde der Rückstand durch Säulenchromatographie auf Kieselgel (Pentan/EtOAc $=2: 1)$ gereinigt. Man erhielt das Domino-Produkt 117 (846 mg, $1.73 \mathrm{mmol}, 78 \%$ ) als hellgelben Feststoff.

$\mathbf{R}_{\mathbf{f}}=0.19-0.36($ Petan $/$ EtOAc $=2: 1)$.

${ }^{1}$ H-NMR (300 MHz, $\mathrm{CDCl}_{3}$, ausgewählt): $\delta=3.41-3.62\left(\mathrm{~m}, 1 \mathrm{H}, \mathrm{OCH}_{\mathrm{a}} \mathrm{H}_{\mathrm{b}} \mathrm{CH}_{3}\right), 3.79-4.02$ $\left(\mathrm{m}, 1 \mathrm{H}, \mathrm{OCH}_{\mathrm{a}} \mathrm{H}_{\mathrm{b}} \mathrm{CH}_{3}\right), 4.68-4.88\left(\mathrm{~m}, 1 \mathrm{H}, \mathrm{NCH}_{\mathrm{a}} \mathrm{H}_{\mathrm{b}}\right)$ 4.92-5.41 (m, $5 \mathrm{H}, \mathrm{NCH}_{\mathrm{a}} \mathrm{H}_{\mathrm{b}}, \mathrm{CH}_{2} \mathrm{Ph}$, 3'”-H, 6-H), 7.29-7.50 (m, 5 H, $5 \times \mathrm{Ph}-\mathrm{H}), 7.55$ ( $\mathrm{m}_{\mathrm{c}}, 1 \mathrm{H}, 7^{\prime}$ '-H), 7.71 ( $\mathrm{m}_{\mathrm{c}}, 1 \mathrm{H}, 6$ '”-H), 7.81 (d, ${ }^{3} J=7.9 \mathrm{~Hz}, 1 \mathrm{H}, 8$ ' '-H), 7.94-8.13 (m, $2 \mathrm{H}, 5$ ' '-H, 9' -H).

MS (ESI): $m / z(\%)=489.2(100)[\mathrm{M}+\mathrm{H}]^{+}$.

$\mathrm{C}_{29} \mathrm{H}_{32} \mathrm{~N}_{2} \mathrm{O}_{5}(488.57)$

Ber.: $\quad 489.2384 \quad[\mathrm{M}+\mathrm{H}]^{+}$

Gef.: $\quad 489.2383$ (ESI-HRMS) 
4.2 4-(2-2,2,2-Trichlor-ethoxycarbonyl-2,3-dihydro-1H-pyrrolo[3,4b]chinolin-3-ylmethyl)-6-ethoxy-5-ethyl-tetrahydropyran-2-on (118)

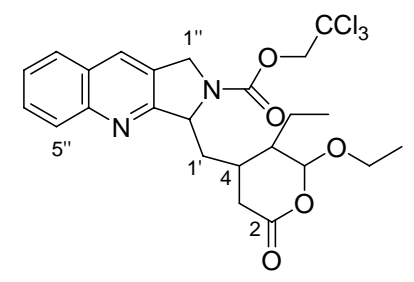

Eine Lösung des Enolethers 64 (99 mg, 0.99 mmol, 4.5 Äq.) in Toluol (2 mL) wurde zu einer Mischung von EDDA (3 mg), 114 (85 mg, 0.22 mmol, 1.0 Äq.) und Meldrum-Säure (32 mg, $0.22 \mathrm{mmol}, 1.0$ Äq.) in einem Druckkolben gegeben. Die Reaktion wurde im Ultraschallbad $\left(58{ }^{\circ} \mathrm{C}\right)$ durchgeführt. Nach $5 \mathrm{~h}$ Reaktionszeit wurde das Reaktionsgemisch über $\mathrm{Na}_{2} \mathrm{SO}_{4}$ getrocknet und filtriert. Nach Entfernung des Lösungsmittels wurde der Rückstand durch Säulenchromatographie auf Kieselgel (Pentan/EtOAc $=3: 1)$ gereinigt. Man erhielt das Domino-Produkt 118 (76 mg, 0.14 mmol, 65\%) als weißen Feststoff.

$\mathbf{R}_{\mathbf{f}}=0.25($ Petan $/$ EtOAc $=3: 1)$.

${ }^{1}$ H-NMR (300 MHz, $\mathrm{CDCl}_{3}$, ausgewählt): $\delta=3.43-3.63\left(\mathrm{~m}, 1 \mathrm{H}, \mathrm{OCH}_{\mathrm{a}} \mathrm{H}_{\mathrm{b}} \mathrm{CH}_{3}\right), 3.78-4.02$ (m, $\left.1 \mathrm{H}, \mathrm{OCH}_{\mathrm{a}} \mathrm{H}_{\mathrm{b}} \mathrm{CH}_{3}\right), 4.73-5.42$ (m, $6 \mathrm{H}, \mathrm{NCH}_{2}, \mathrm{CH}_{2} \mathrm{Cl}_{3}, 3$ '’-H, 6-H), 7.57 (m $\mathrm{c}, 1 \mathrm{H}, 7$ '’-H), $7.73\left(\mathrm{~m}_{\mathrm{c}}, 1 \mathrm{H}, 6\right.$ ' '-H), 7.84 (d, ${ }^{3} \mathrm{~J}=8.0 \mathrm{~Hz}, 1 \mathrm{H}, 8^{\prime}$ '-H), 8.02-8.12 (m, 2 H, 5' -H, 9' -H). MS (ESI): $m / z(\%)=531.1(96), 529.1(100)[\mathrm{M}+\mathrm{H}]^{+}$.

$\mathbf{C}_{24} \mathbf{H}_{27} \mathbf{C l}_{3} \mathbf{N}_{2} \mathbf{O}_{5}$ (529.84)

Ber.:

$529.1058 \quad[\mathrm{M}+\mathrm{H}]^{+}$

531.1028

Gef:

529.1058 (ESI-HRMS)

531.1028 


\section{$4.3 \quad 2-(9-O x o-5 b, 6,7,8,9,11-h e x a h y d r o-i n d o l i z i n o[1,2-b] c h i n o l i n-7-y l)-$ butyraldehyd (61)}

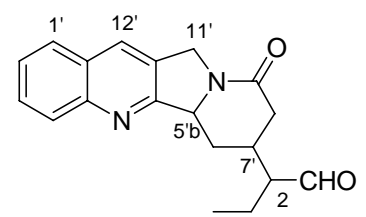

\section{Ausgehend von dem Dominoprodukt 117 über eine Stufe}

Eine Suspension von 117 (330 mg, 0.675 mmol, 1.00 Äq.) und Palladium auf Aktivkohle $(10 \% \mathrm{Pd}, 72 \mathrm{mg})$ in Ethanol $(30 \mathrm{~mL})$ wurde bei $\mathrm{RT}$ unter $\mathrm{H}_{2}$-Atmosphäre $\left(\mathrm{H}_{2}\right.$-Ballon) für 1.5 h gerührt. Dann wurde die Reaktionsmischung über Celite ${ }^{\circledR}$ filtriert und die Mutterlauge im Vakuum konzentriert. Der Rückstand wurde durch Säulenchromatographie auf Kieselgel $\left(\right.$ EtOAc $\left./ \mathrm{NEt}_{3}=20: 1\right)$ gereinigt. Man erhielt den Aldehyd $61(158 \mathrm{mg}, 0.512 \mathrm{mmol}, 76 \%)$ als sehr hellbraunen Feststoff.

\section{Ausgehend von dem Aldehyd 114 über zwei Stufen}

Eine Mischung von 64 (84 mg, 0.84 mmol, 2.0 Äq.), EDDA (eine Spatelspitze), 114 (0.16 g, $0.42 \mathrm{mmol}, 1.0$ Äq.) und Meldrum-Säure (61 mg, $0.42 \mathrm{mmol}, 1.0$ Äq.) in Toluol (5 mL) wurde bei RT für $3 \mathrm{~h}$ gerührt. Konzentration der Reaktionsmischung im Vakuum lieferte das Rohprodukt von 118 (243 mg, 0.46 mmol, 109\%) als gelben Feststoff.

Ein Teil des Rohproduktes von 118 (36 mg) wurde zu einer Mischung von Zn-Pulver (186 $\mathrm{mg})$ in THF $(3 \mathrm{~mL})$ und Wasser $(0.5 \mathrm{~mL})$ gegeben. Nach $2 \mathrm{~h}$ Rühren bei RT wurde die Reaktionsmischung bei $40{ }^{\circ} \mathrm{C}$ für $8 \mathrm{~h}$ erhitzt. Nach Abkühlung auf RT wurde Zn-Pulver über Celite $^{\circledR}$ abfiltriert und die Mutterlauge im Vakuum konzentriert. Der Rückstand wurde durch Säulenchromatographie auf Kieselgel $($ Petan/EtOAc $=2: 1 \rightarrow$ EtOAc) gereinigt. Man erhielt den Aldehyd 61 (7.8 mg, 25 mmol, 41\% über zwei Stufen) als sehr hellbraunen Feststoff.

$\mathbf{R}_{\mathbf{f}}=0.35\left(\mathrm{EtOAc} / \mathrm{NEt}_{3}=20: 1\right)$.

${ }^{1}$ H-NMR (300 MHz, $\mathrm{CDCl}_{3}$, ausgewählt, 4 Diastereomer A:B:C:D = 1:1:1:1): $\delta=0.86-1.07$ (m, $\left.3 \mathrm{H}, \mathrm{CH}_{3}\right), 1.63-1.92$ (m, 2 H, $\left.\mathrm{CH}_{2} \mathrm{CH}_{3}\right), 4.58-4.76$ (m, $\left.1 \mathrm{H}, 11^{\prime}-\mathrm{H}\right), 4.85-5.04$ (m, $1 \mathrm{H}$, 5 'b-H), 5.15 (d, ${ }^{2} J=7.8 \mathrm{~Hz}, 0.25 \mathrm{H}, 11$ '-H, Diastereomer A), 5.21 (d, ${ }^{2} J=7.7 \mathrm{~Hz}, 0.25 \mathrm{H}$, 11'-H, Diastereomer B), 5.31 (d, ${ }^{2} J=16.3 \mathrm{~Hz}, 0.50 \mathrm{H}, 11^{\prime}$-H, Diastereomer C und D), 7.56 $\left(\mathrm{m}_{\mathrm{c}}, 1 \mathrm{H}, 2^{\prime}-\mathrm{H}\right), 7.72\left(\mathrm{~m}_{\mathrm{c}}, 1 \mathrm{H}, 3^{\prime}-\mathrm{H}\right), 7.83\left(\mathrm{~d},{ }^{3} \mathrm{~J}=8.1 \mathrm{~Hz}, 1 \mathrm{H}, 1^{\prime}-\mathrm{H}\right), 8.00-8.15$ (m, $2 \mathrm{H}$, 4'-H, 12'-H), 9.67-9.76 (m, 1 H, CHO). 
${ }^{13}$ C-NMR (125 MHz, $\mathrm{CDCl}_{3}$, ausgewählt): $\delta=11.33,11.39,11.47,11.64\left(\mathrm{CH}_{3}\right), 19.03$, 19.25, 19.79, $19.81\left(\mathrm{CH}_{2} \mathrm{CH}_{3}\right), 161.4,161.5,162.0,162.1$ (C-5'a), 168.1, 168.1, 169.6, 169.8 (NCO), 203.4, 203.6, 203.7 (CHO).

MS (ESI): $m / z(\%)=331.1(98)[\mathrm{M}+\mathrm{Na}]^{+}, 309.1(100)[\mathrm{M}+\mathrm{H}]^{+}$.

$\mathbf{C}_{19} \mathbf{H}_{20} \mathbf{N}_{2} \mathbf{O}_{2}(308.37)$

Ber.: $\quad 309.1597 \quad[\mathrm{M}+\mathrm{H}]^{+}$

$331.1417 \quad[\mathrm{M}+\mathrm{Na}]^{+}$

Gef.: $\quad 309.1597$ (ESI-HRMS)

331.1417 


\section{Aufbau des Lactonrings und Synthese von Camptothecin}

\section{$5.12-(9-O x 0-5 b, 6,7,8,9,11-h e x a h y d r o-i n d o l i z i n o[1,2-b] c h i n o l i n-7-y l)-$ buttersäure (129)}

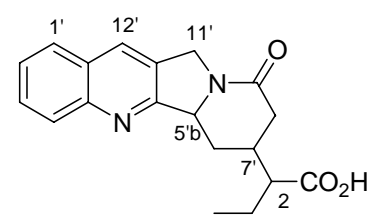

Zu einer Mischung von 61 (134 mg, 0.434 mmol, 1.00 Äq.), 2-Methyl-2-buten (3.50 mL) in tert-BuOH $(8 \mathrm{~mL})$ und Wasser $(2 \mathrm{~mL})$ bei RT wurde $\mathrm{NaH}_{2} \mathrm{PO}_{4} \cdot \mathrm{H}_{2} \mathrm{O}(599 \mathrm{mg}, 4.34 \mathrm{mmol}$, 10.0 Äq.) und $\mathrm{NaClO}_{2}$ (80\% tech., $490 \mathrm{mg}, 4.34$ mmol, 10.0 Äq.) gegeben. Nach $25 \mathrm{~min}$ Rühren wurden $5 \mathrm{~mL}$ einer Pufferlösung $(\mathrm{pH}=7)$ hingefügt. Die wäßrige Phase wurde mit EtOAc $(3 \times 30 \mathrm{~mL})$ extrahiert. Die vereinigten organischen Phasen wurden mit gesättiger $\mathrm{NaCl}$-Lösung gewaschen, über $\mathrm{MgSO}_{4}$ getrocknet und filtriert. Nach Entfernung des Lösungsmittels erhielt man die Carbonsäure 129 (122 mg, $0.376 \mathrm{mmol}, 90 \%$ ) als weißen Feststoff.

$\mathbf{R}_{\mathbf{f}}=0.31(\mathrm{EtOAc} / \mathrm{HOAc}=30: 1)$.

MS (ESI): $m / z(\%)=325.1(100)[\mathrm{M}+\mathrm{H}]^{+}$.

$\mathrm{C}_{19} \mathbf{H}_{20} \mathbf{N}_{2} \mathbf{O}_{3}(324.37)$

Ber.: $\quad 325.1546[\mathrm{M}+\mathrm{H}]^{+}$

Gef.: $\quad 325.1547$ (ESI-HRMS) 


\subsection{7-Propionyl-11H-indolizino[1,2-b]chinolin-9-on (123)}

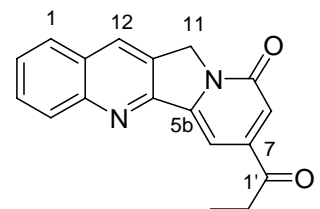

Zu einer Lösung des Aldehyds 61 (39 mg, 0.13 mmol, 1.0 Äq.) in 1,4-Dioxan (1.3 mL) wurde DDQ (57 mg, $0.25 \mathrm{mmol}, 2.0$ Äq.) gegeben. Es wurde nachfolgend bei $50{ }^{\circ} \mathrm{C}, 80^{\circ} \mathrm{C}$ und $100{ }^{\circ} \mathrm{C}$ jeweils für $1 \mathrm{~h}$ gerührt, wobei sich Niederschlag bildete. Nach Abkühlung auf RT wurde der Niederschlag abfiltriert und mit $\mathrm{CH}_{2} \mathrm{Cl}_{2}$ gewaschen. Die Mutterlauge wurde im Vakuum konzentriert und der Rückstand durch Säulenchromatographie auf Kieselgel $\left(\mathrm{CH}_{2} \mathrm{Cl}_{2} / \mathrm{MeOH}=20: 1\right)$ gereinigt. Man erhielt zwei Fraktionen bzw. das Keton 123 (12 mg, $41 \mu \mathrm{mol}, 33 \%)$ als braunen Feststoff und den Aldehyd 122 (21 mg, $69 \mu \mathrm{mol}, 55 \%)$ als hellgelben Feststoff.

$\mathbf{R}_{\mathbf{f}}=0.35\left(\mathrm{CH}_{2} \mathrm{Cl}_{2} / \mathrm{MeOH}=20: 1\right)$.

${ }^{1} \mathbf{H}-\mathbf{N M R}\left(300 \mathrm{MHz}, \mathrm{CDCl}_{3}\right): \delta=1.26\left(\mathrm{t},{ }^{3} \mathrm{~J}=7.2 \mathrm{~Hz}, 3 \mathrm{H}, \mathrm{CH}_{3}\right), 3.01$ (q, J = 7.2 Hz, $2 \mathrm{H}$, $\left.\mathrm{CH}_{2} \mathrm{CH}_{3}\right), 5.24(\mathrm{~s}, 2 \mathrm{H}, 11-\mathrm{H}), 7.16\left(\mathrm{~d},{ }^{4} \mathrm{~J}=1.4 \mathrm{~Hz}, 1 \mathrm{H}, 6-\mathrm{H}\right), 7.60-7.68(\mathrm{~m}, 2 \mathrm{H}, 8-\mathrm{H}, 2-\mathrm{H})$, $7.81\left(\mathrm{~m}_{\mathrm{c}}, 1 \mathrm{H}, 3-\mathrm{H}\right), 7.89\left(\mathrm{~d},{ }^{3} \mathrm{~J}=8.1 \mathrm{~Hz}, 1 \mathrm{H}, 1-\mathrm{H}\right), 8.20\left(\mathrm{~d},{ }^{3} \mathrm{~J}=8.5 \mathrm{~Hz}, 1 \mathrm{H}, 4-\mathrm{H}\right), 8.34$ (s, 1 H, 12-H).

MS (ESI): $m / z(\%)=291.1(100)[\mathrm{M}+\mathrm{H}]^{+}$.

$\mathbf{C}_{18} \mathbf{H}_{14} \mathbf{N}_{2} \mathbf{O}_{2}(290.32)$

Ber.: $\quad 291.1128[\mathrm{M}+\mathrm{H}]^{+}$

Gef.: $\quad 291.1126$ (ESI-HRMS) 


\subsection{2-(9-Oxo-9,11-dihydro-indolizino[1,2-b]chinolin-7-yl)-butyraldehyd} (122)

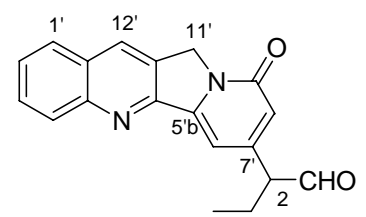

(Durchführung siehe $\mathbf{5 . 2}$ bei der Synthese von 123).

$\mathbf{R}_{\mathbf{f}}=0.21\left(\mathrm{CH}_{2} \mathrm{Cl}_{2} / \mathrm{MeOH}=20: 1\right)$.

${ }^{1}$ H-NMR $\left(600 \mathrm{MHz}, \mathrm{CDCl}_{3}\right): \delta=1.00\left(\mathrm{t}, J=7.4 \mathrm{~Hz}, 3 \mathrm{H}, \mathrm{CH}_{3}\right), 1.82-1.98(\mathrm{~m}, 1 \mathrm{H}$, $\left.\mathrm{CH}_{\mathrm{a}} \mathrm{H}_{\mathrm{b}} \mathrm{CH}_{3}\right), 2.20\left(\mathrm{~m}_{\mathrm{c}}, 1 \mathrm{H}, \mathrm{CH}_{\mathrm{a}} \mathrm{H}_{\mathrm{b}} \mathrm{CH}_{3}\right), 3.46\left(\mathrm{~m}_{\mathrm{c}}, 1 \mathrm{H}, 2-\mathrm{H}\right), 5.29$ (s, $2 \mathrm{H}, 11$ '- $\left.\mathrm{H}\right), 6.62\left(\mathrm{~d},{ }^{4} J\right.$ $\left.=1.4 \mathrm{~Hz}, 1 \mathrm{H}, 8^{\prime}-\mathrm{H}\right), 7.21\left(\mathrm{~d},{ }^{4} \mathrm{~J}=1.4 \mathrm{~Hz}, 1 \mathrm{H}, 6{ }^{\prime}-\mathrm{H}\right), 7.66\left(\mathrm{~m}_{\mathrm{c}}, 1 \mathrm{H}, 2^{\prime}-\mathrm{H}\right), 7.83\left(\mathrm{~m}_{\mathrm{c}}, 1 \mathrm{H}\right.$, 3'-H), 7.94 (d, $\left.{ }^{3} J=8.2 \mathrm{~Hz}, 1 \mathrm{H}, 1^{\prime}-\mathrm{H}\right), 8.22$ (d, $\left.{ }^{3} \mathrm{~J}=8.6 \mathrm{~Hz}, 1 \mathrm{H}, 4^{\prime}-\mathrm{H}\right), 8.40$ (s, $\left.1 \mathrm{H}, 12^{\prime}-\mathrm{H}\right)$, $9.76\left(\mathrm{~d},{ }^{3} \mathrm{~J}=1.7 \mathrm{~Hz}, 1 \mathrm{H}, \mathrm{CHO}\right)$.

${ }^{13} \mathrm{C}-\mathrm{NMR}\left(125 \mathrm{MHz}, \mathrm{CDCl}_{3}\right): \delta=11.68\left(\mathrm{CH}_{3}\right), 22.31\left(\mathrm{CH}_{2} \mathrm{CH}_{3}\right), 49.81(\mathrm{C}-11$ '), $60.50(\mathrm{C}-2)$, 101.1 (C-6'), 120.1 (C-8'), 127.9 (C-2'), 128.1 (C-1'), 128.1 (C-12'a '), 128.8 (C-11'a*), 129.6 (C-4'), 130.5 (C-3’), 131.1 (C-12'), 146.4 (C-5’b), 148.8 (C-7'), 150.6 (C-4'a), 152.6 (C-5'a), 161.2 (C-9'), 199.1 (CHO).

MS (ESI): $m / z(\%)=327.1(55)[\mathrm{M}+\mathrm{Na}]^{+}, 305.1(100)[\mathrm{M}+\mathrm{H}]^{+}$.

$\mathbf{C}_{19} \mathbf{H}_{16} \mathbf{N}_{2} \mathbf{O}_{2}(304.34)$

Ber.: $\quad 305.1284 \quad[\mathrm{M}+\mathrm{H}]^{+}$ $327.1104 \quad[\mathrm{M}+\mathrm{Na}]^{+}$

Gef.: $\quad 305.1284$ (ESI-HRMS) 327.1104 


\subsection{2-(9-Oxo-5b,6,7,8,9,11-hexahydro-indolizino[1,2-b]chinolin-7-yl)- buttersäuremethylester (132)}

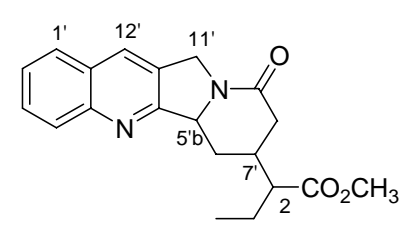

Ausgehend von dem Aldehyd 61 über zwei Stufen

$\mathrm{Zu}$ einer Mischung von 61 (96 mg, $0.31 \mathrm{mmol}, 1.0$ Äq.), 2-Methyl-2-buten (2.5 mL) in tert$\mathrm{BuOH}(6 \mathrm{~mL})$ und Wasser $(1.5 \mathrm{~mL})$ bei RT wurde $\mathrm{NaH}_{2} \mathrm{PO}_{4} \cdot \mathrm{H}_{2} \mathrm{O}(0.43 \mathrm{~g}, 3.1 \mathrm{mmol}, 10$ Äq. $)$ und $\mathrm{NaClO}_{2}$ (80\% tech., $0.35 \mathrm{~g}, 3.1 \mathrm{mmol}, 10$ Äq.) gegeben. Nach 40 min Rühren wurden $4 \mathrm{~mL}$ einer Pufferlösung ( $\mathrm{pH}=7)$ und Wasser $(4 \mathrm{~mL})$ hingefügt. Die wäßrige Phase wurde mit $\mathrm{CH}_{2} \mathrm{Cl}_{2}(3 \times 20 \mathrm{~mL})$ extrahiert. Die vereinigten organischen Phasen wurden über $\mathrm{MgSO}_{4}$ getrocknet und filtriert. Nach Entfernung des Lösungsmittels erhielt man die Carbonsäure 129 $(0.11 \mathrm{mg})$ als weißen Feststoff.

Die Carbonsäure 129 (0.11 mg) wurde ohne weitere Reinigung in Methanol (2.8 mL) und Toluol (10 mL) aufgenommen. Dann wurde TMS-CHN 2 (2 $\mathrm{M}$ in $\mathrm{Et}_{2} \mathrm{O}, 0.20 \mathrm{~mL}, 0.40 \mathrm{mmol}$, 1.3 Äq.) bei RT zugetropft. Nach 45 min Rühren wurde die Reaktionsmischung im Vakuum konzentriert. Der Rückstand wurde durch Säulenchromatographie auf Kieselgel (EtOAc/NEt 3 = 200:9) gereinigt. Man erhielt den Methylester 132 (88 mg, $0.26 \mathrm{mmol}, 84 \%$ über zwei Stufen).

$\mathbf{R}_{\mathbf{f}}=0.38\left(\mathrm{EtOAc} / \mathrm{NEt}_{3}=20: 1\right)$.

${ }^{1}$ H-NMR (300 MHz, $\mathrm{CDCl}_{3}$, ausgewählt, 4 Diastereomer A:B:C:D = 1:1:1:1): $\delta=0.95\left(\mathrm{~m}_{\mathrm{c}}\right.$, $3 \mathrm{H}, \mathrm{CH}_{2} \mathrm{CH}_{3}$ ), 1.64-1.89 (m, $\left.2 \mathrm{H}, \mathrm{CH}_{2} \mathrm{CH}_{3}\right), 3.72$ (s, $0.75 \mathrm{H}, \mathrm{CO}_{2} \mathrm{CH}_{3}$, Diastereomer A), 3.74 (s, $0.75 \mathrm{H}, \mathrm{CO}_{2} \mathrm{CH}_{3}$, Diastereomer B), 3.75 (s, $0.75 \mathrm{H}, \mathrm{CO}_{2} \mathrm{CH}_{3}$, Diastereomer C), 3.77 (s, $0.75 \mathrm{H}, \mathrm{CO}_{2} \mathrm{CH}_{3}$, Diastereomer D), $4.66\left(\mathrm{~m}_{\mathrm{c}}, 1 \mathrm{H}, 11^{\prime}-\mathrm{H}\right), 4.83-5.03$ (m, $1 \mathrm{H}, 5$ 'b-H), 5.16 (d, ${ }^{2} J=5.4 \mathrm{~Hz}, 0.25 \mathrm{H}, 11$ ' $-\mathrm{H}$, Diastereomer A), $5.21\left(\mathrm{~d},{ }^{2} J=5.4 \mathrm{~Hz}, 0.25 \mathrm{H}, 11\right.$ ' $-\mathrm{H}$, Diastereomer B), 5.30 (d, ${ }^{2} J=16.5 \mathrm{~Hz}, 0.50 \mathrm{H}, 11^{\prime}-\mathrm{H}$, Diastereomer C und D), $7.56\left(\mathrm{~m}_{\mathrm{c}}, 1 \mathrm{H}\right.$, 2'-H), 7.72 (m $\left.\mathrm{m}_{\mathrm{c}}, 1 \mathrm{H}, 3^{\prime}-\mathrm{H}\right), 7.83$ (d, ${ }^{3} \mathrm{~J}=7.9 \mathrm{~Hz}, 1 \mathrm{H}, 1$ '-H), 8.04-8.12 (m, 2 H, 4'-H, 12'-H). ${ }^{13}$ C-NMR (125 MHz, $\mathrm{CDCl}_{3}$, ausgewählt): $\delta=11.71,11.73,11.75,11.82\left(\mathrm{CH}_{2} \mathrm{CH}_{3}\right), 22.61$, 22.80, 23.06, $23.34\left(\mathrm{CH}_{2} \mathrm{CH}_{3}\right), 161.6,161.7,162.2,162.3$ (C-5'a), 168.4, $169.8,169.9$ (NCO), 174.8, 174.9, $175.1\left(\mathrm{CO}_{2} \mathrm{CH}_{3}\right)$.

MS (ESI): $m / z(\%)=339.1(100)[\mathrm{M}+\mathrm{H}]^{+}$. 
$\mathrm{C}_{20} \mathrm{H}_{22} \mathrm{~N}_{2} \mathbf{O}_{3}(338.40)$

Ber.:

$339.1703 \quad[\mathrm{M}+\mathrm{H}]^{+}$

$361.1522[\mathrm{M}+\mathrm{Na}]^{+}$

$377.1262[\mathrm{M}+\mathrm{K}]^{+}$

Gef.: $\quad 339.1702$ (ESI-HRMS)

361.1521

377.1259

\subsection{2-(9-Oxo-9,11-dihydro-indolizino[1,2-b]chinolin-7-yl)-buttersäure- methylester (20)}

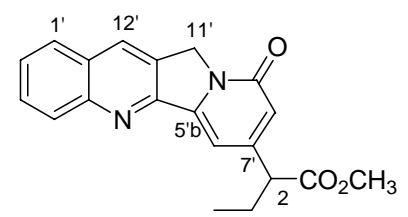

Der Methylester 132 (52 mg, $0.16 \mathrm{mmol}, 1.0$ Äq.) und DDQ (80 mg, $0.35 \mathrm{mmol}, 2.2$ Äq.) wurden in 1,4-Dioxan $(1.5 \mathrm{~mL})$ gelöst. Es wurde bei $60^{\circ} \mathrm{C}$ für $40 \mathrm{~min}$ erhitzt, wobei sich Niederschlag bildete. Nach Abkühlung auf RT wurde der Niederschlag abfiltriert und mit $\mathrm{CH}_{2} \mathrm{Cl}_{2}$ gewaschen. Die Mutterlauge wurde im Vakuum konzentriert und der Rückstand durch Säulenchromatographie auf Kieselgel $\left(\right.$ EtOAc/ $\left.\mathrm{NEt}_{3}=20: 1\right)$ gereinigt. Man erhielt den Ester 20 (48 mg, $0.14 \mathrm{mmol}, 90 \%$ ) als hellgelben Feststoff.

$\mathbf{R}_{\mathbf{f}}=0.32(\mathrm{EtOAc} / \mathrm{MeOH}=20: 1)$.

${ }^{1} \mathbf{H}-\mathbf{N M R}\left(300 \mathrm{MHz}, \mathrm{CDCl}_{3}\right): \delta=0.93\left(\mathrm{t},{ }^{3} \mathrm{~J}=7.3 \mathrm{~Hz}, 3 \mathrm{H}, \mathrm{CH}_{2} \mathrm{CH}_{3}\right), 1.79-1.97(\mathrm{~m}, 1 \mathrm{H}$, $\left.\mathrm{CH}_{\mathrm{a}} \mathrm{H}_{\mathrm{b}} \mathrm{CH}_{3}\right), 2.05-2.22\left(\mathrm{~m}, 1 \mathrm{H}, \mathrm{CH}_{\mathrm{a}} \mathrm{H}_{\mathrm{b}} \mathrm{CH}_{3}\right), 3.45\left(\mathrm{t},{ }^{3} \mathrm{~J}=7.7 \mathrm{~Hz}, 1 \mathrm{H}, 2-\mathrm{H}\right), 3.69(\mathrm{~s}, 3 \mathrm{H}$, $\left.\mathrm{CO}_{2} \mathrm{CH}_{3}\right), 5.18\left(\mathrm{~s}, 2 \mathrm{H}, 11^{\prime}-\mathrm{H}\right), 6.61\left(\mathrm{t},{ }^{4} \mathrm{~J}=1.3 \mathrm{~Hz}, 1 \mathrm{H}, 88^{\prime}-\mathrm{H}\right), 7.27\left(\mathrm{~d},{ }^{4} \mathrm{~J}=1.3 \mathrm{~Hz}, 1 \mathrm{H}\right.$, 6'-H), 7.58 ( $\left.\mathrm{m}_{\mathrm{c}}, 1 \mathrm{H}, 2^{\prime}-\mathrm{H}\right), 7.76$ (m $\left.\mathrm{c}, 1 \mathrm{H}, 3^{\prime}-\mathrm{H}\right), 7.86$ (d, $\left.{ }^{3} J=8.2 \mathrm{~Hz}, 1 \mathrm{H}, 1^{\prime}-\mathrm{H}\right), 8.16$ (d, ${ }^{3} J$ $\left.=8.6 \mathrm{~Hz}, 1 \mathrm{H}, 4^{\prime}-\mathrm{H}\right), 8.30\left(\mathrm{~s}, 1 \mathrm{H}, 12^{\prime}-\mathrm{H}\right)$.

MS (ESI): $m / z(\%)=335.1(100)[\mathrm{M}+\mathrm{H}]^{+}$.

$\mathrm{C}_{20} \mathrm{H}_{18} \mathbf{N}_{2} \mathbf{O}_{3}(334.37)$

Ber.: $\quad 335.1390$

$[\mathrm{M}+\mathrm{H}]^{+}$

Gef.: $\quad 335.1390$

(ESI-HRMS) 


\section{$5.6 \quad 20-D e s o x y c a m p t o t h e c i n ~(141)$}

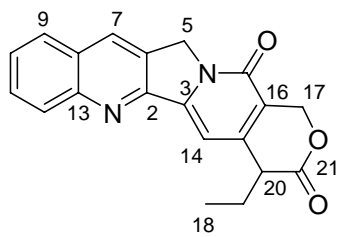

Eine Mischung von 20 (0.11 g, 0.33 mmol, 1.0 Äq.), konz. Schwefelsäure (66 mg), Paraformaldehyd $(78 \mathrm{mg})$ und Wasser $(0.40 \mathrm{~mL})$ in 1,4-Dioxan $(11 \mathrm{~mL})$ im Druckkolben wurde bei $95^{\circ} \mathrm{C}$ für $17 \mathrm{~h}$ erhitzt. Nach Abkühlung auf RT wurde halbgesättige NaCl-Lösung $(10 \mathrm{~mL})$ hingefügt und die wäßrige Phase mit $\mathrm{CH}_{2} \mathrm{Cl}_{2}(3 \times 25 \mathrm{~mL})$ extrahiert. Die vereinigten organischen Phasen wurden über $\mathrm{MgSO}_{4}$ getrocknet, filtriert und im Vakuum konzentriert. Der Rückstand wurde in $\mathrm{CH}_{2} \mathrm{Cl}_{2}(10 \mathrm{~mL})$ aufgenommen. Der unlösliche Feststoff wurde abfiltriert und mit $\mathrm{CH}_{2} \mathrm{Cl}_{2}$ gewaschen. Weitere Trocknung des Feststoffs im Hockvakuum lieferte die Carbonsäure 143 (40 mg, 0.12 mmol, 38\%) als gelben Feststoff. Die Mutterlauge wurde konzentriert und der Rückstand wurde durch Säulenchromatographie auf Kieselgel $\left(\mathrm{CH}_{2} \mathrm{Cl}_{2} / \mathrm{MeOH}=20: 1\right)$ gereinigt. Man erhielt zwei Fraktionen bzw. das Lacton 141 (26 mg, $78 \mu \mathrm{mol}, 24 \%)$ als gelben Feststoff und den Alkohol 142 (8.2 mg, $23 \mu \mathrm{mol}, 7 \%)$ als gelbes Ö1.

Ein Teil des erhaltenen Lactons 141 (16 mg) wurde wiederum durch Säulenchromatographie auf Kieselgel (EtOAc/MeOH = 40:1) gereinigt. Somit wurde das Lacton 141 (6.3 mg, 9\% bezogen auf das Edukt) in viel reinerer Form gewonnen.

$\mathbf{R}_{\mathbf{f}}=0.33\left(\mathrm{CH}_{2} \mathrm{Cl}_{2} / \mathrm{MeOH}=20: 1\right)$ oder $0.35(\mathrm{EtOAc} / \mathrm{MeOH}=40: 1)$.

${ }^{1} \mathbf{H}-\mathrm{NMR}\left(300 \mathrm{MHz}, \mathrm{CDCl}_{3}\right): \delta=1.10\left(\mathrm{t},{ }^{3} \mathrm{~J}=7.3 \mathrm{~Hz}, 3 \mathrm{H}, \mathrm{CH}_{2} \mathrm{CH}_{3}\right), 2.03-2.18(\mathrm{~m}, 2 \mathrm{H}$, $\left.\mathrm{CH}_{2} \mathrm{CH}_{3}\right), 3.64\left(\mathrm{t},{ }^{3} \mathrm{~J}=6.6 \mathrm{~Hz}, 1 \mathrm{H}, 20-\mathrm{H}\right), 5.31(\mathrm{~s}, 2 \mathrm{H}, 5-\mathrm{H}), 5.40\left(\mathrm{~d},{ }^{2} \mathrm{~J}=16.3 \mathrm{~Hz}, 1 \mathrm{H}\right.$, $\left.17-\mathrm{H}_{\mathrm{a}}\right), 5.58\left(\mathrm{~d},{ }^{2} \mathrm{~J}=16.3 \mathrm{~Hz}, 1 \mathrm{H}, 17-\mathrm{H}_{\mathrm{b}}\right), 7.19(\mathrm{~s}, 1 \mathrm{H}, 14-\mathrm{H}), 7.67\left(\mathrm{~m}_{\mathrm{c}}, 1 \mathrm{H}, 10-\mathrm{H}\right), 7.84\left(\mathrm{~m}_{\mathrm{c}}\right.$, $1 \mathrm{H}, 11-\mathrm{H}), 7.95$ (d, $\left.{ }^{3} \mathrm{~J}=8.3 \mathrm{~Hz}, 1 \mathrm{H}, 9-\mathrm{H}\right), 8.22$ (d, $\left.{ }^{3} J=8.5 \mathrm{~Hz}, 1 \mathrm{H}, 12-\mathrm{H}\right), 8.40$ (s, $1 \mathrm{H}$, 7-H).

MS (ESI): $m / z(\%)=333.1(100)[\mathrm{M}+\mathrm{H}]^{+}$.

$\mathbf{C}_{20} \mathbf{H}_{16} \mathbf{N}_{2} \mathbf{O}_{3}(332.35)$

Ber.: $\quad 333.1233 \quad[\mathrm{M}+\mathrm{H}]^{+}$

Gef.: $\quad 333.1234$ (ESI-HRMS) 


\subsection{4-Ethyl-4-hydroxymethyl-1,7-dihydro-4H-2-oxa-6a,13-diaza- dibenzo[b,g]-fluoren-3,6-dion (142)}

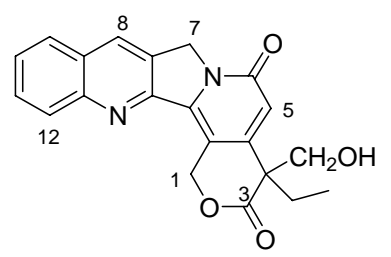

(Durchführung siehe 5.6 bei der Synthese von 141).

$\mathbf{R}_{\mathbf{f}}=0.23\left(\mathrm{CH}_{2} \mathrm{Cl}_{2} / \mathrm{MeOH}=20: 1\right)$.

${ }^{1} \mathbf{H}-\mathbf{N M R}\left(300 \mathrm{MHz}, \mathrm{CDCl}_{3}\right): \delta=0.88\left(\mathrm{t},{ }^{3} \mathrm{~J}=7.2 \mathrm{~Hz}, 3 \mathrm{H}, \mathrm{CH}_{2} \mathrm{CH}_{3}\right), 1.84-2.01(\mathrm{~m}, 1 \mathrm{H}$, $\left.\mathrm{CH}_{\mathrm{a}} \mathrm{H}_{\mathrm{b}} \mathrm{CH}_{3}\right), 2.06-2.25\left(\mathrm{~m}, 1 \mathrm{H}, \mathrm{CH}_{\mathrm{a}} \mathrm{H}_{\mathrm{b}} \mathrm{CH}_{3}\right), 4.02\left(\mathrm{~d},{ }^{2} \mathrm{~J}=10.4 \mathrm{~Hz}, 1 \mathrm{H}, \mathrm{CH}_{\mathrm{a}} \mathrm{H}_{\mathrm{b}} \mathrm{OH}\right), 4.32(\mathrm{~d}$, $\left.{ }^{2} J=10.4 \mathrm{~Hz}, 1 \mathrm{H}, \mathrm{CH}_{\mathrm{a}} \mathrm{H}_{\mathrm{b}} \mathrm{OH}\right), 5.07\left(\mathrm{~m}_{\mathrm{c}}, 2 \mathrm{H}, 7-\mathrm{H}\right), 6.13\left(\mathrm{~d},{ }^{2} J=15.4 \mathrm{~Hz}, 1 \mathrm{H}, 1-\mathrm{H}_{\mathrm{a}}\right), 6.33(\mathrm{~d}$, $\left.{ }^{2} J=15.4 \mathrm{~Hz}, 1 \mathrm{H}, 1-\mathrm{H}_{\mathrm{b}}\right), 6.67(\mathrm{~s}, 1 \mathrm{H}, 5-\mathrm{H}), 7.54\left(\mathrm{~m}_{\mathrm{c}}, 1 \mathrm{H}, 10-\mathrm{H}\right), 7.65-7.84(\mathrm{~m}, 2 \mathrm{H}, 9-\mathrm{H}$, 11-H), 8.03-8.22 (m, 2 H, 8-H, 12-H).

MS (ESI): $m / z(\%)=363.1(100)[\mathrm{M}+\mathrm{H}]^{+}$.

$\mathrm{C}_{21} \mathrm{H}_{18} \mathrm{~N}_{2} \mathrm{O}_{4}(362.38)$

Ber.: $\quad 363.1339 \quad[\mathrm{M}+\mathrm{H}]^{+}$

Gef.: $\quad 363.1339$ (ESI-HRMS) 


\subsection{2-(9-Oxo-9,11-dihydro-indolizino[1,2-b]chinolin-7-yl)-buttersäure} (143)

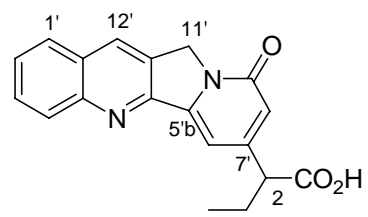

(Durchführung siehe 5.6 bei der Synthese von 141).

${ }^{1}$ H-NMR (300 MHz, d $\mathrm{d}_{6}$-DMSO): $\delta=0.91\left(\mathrm{t},{ }^{3} J=7.2 \mathrm{~Hz}, 3 \mathrm{H}, \mathrm{CH}_{3}\right), 1.67-1.86(\mathrm{~m}, 1 \mathrm{H}$, $\left.\mathrm{CH}_{\mathrm{a}} \mathrm{H}_{\mathrm{b}} \mathrm{CH}_{3}\right), 1.93-2.10\left(\mathrm{~m}, 1 \mathrm{H}, \mathrm{CH}_{\mathrm{a}} \mathrm{H}_{\mathrm{b}} \mathrm{CH}_{3}\right), 3.48-3.61$ (m, $\left.1 \mathrm{H}, 2-\mathrm{H}\right), 5.22\left(\mathrm{~s}, 2 \mathrm{H}, 11^{\prime}-\mathrm{H}\right)$, $6.50\left(\mathrm{~d},{ }^{4} \mathrm{~J}=1.0 \mathrm{~Hz}, 1 \mathrm{H}, 8^{\prime}-\mathrm{H}\right), 7.16\left(\mathrm{~d},{ }^{4} J=1.0 \mathrm{~Hz}, 1 \mathrm{H}, 6^{\prime}-\mathrm{H}\right), 7.70\left(\mathrm{~m}_{\mathrm{c}}, 1 \mathrm{H}, 2^{\prime}-\mathrm{H}\right), 7.85$ (m $\left.\mathrm{m}_{\mathrm{c}}, 1 \mathrm{H}, 3^{\prime}-\mathrm{H}\right), 8.07-8.22$ (m, 2 H, 1'-H, 4'-H), 8.67 (s, 1 H, 12'-H), 11.98 (br, 1 H, COOH). MS (ESI): $m / z(\%)=321.1(100)[\mathrm{M}+\mathrm{H}]^{+}$.

$\mathrm{C}_{19} \mathrm{H}_{16} \mathrm{~N}_{2} \mathrm{O}_{3}(320.34)$

Ber.: $\quad 321.1233 \quad[\mathrm{M}+\mathrm{H}]^{+}$

Gef.: $\quad 321.1233$ (ESI-HRMS) 


\section{$5.9 \quad$ ( \pm )-Camptothecin (11)}

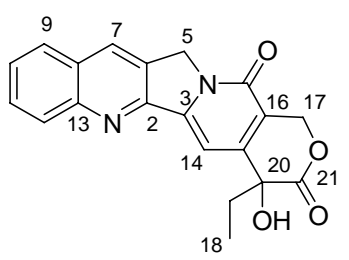

$\mathrm{Zu}$ einer Lösung von 141 (6.3 mg, $19 \mu \mathrm{mol}$, 1.00 Äq.), 25\%-wäßrige $\mathrm{Me}_{2} \mathrm{NH}$-Lösung (19 mg), $\mathrm{CuCl}_{2}(10 \mathrm{mg})$ in DMF $(1 \mathrm{~mL})$ bei RT wurde Sauerstoff $\left(\mathrm{O}_{2}\right.$-Ballon) für $7 \mathrm{~h}$ eingeleitet. Anschließend wurden Wasser (5 mL) und ein Tropfen Salzsäure (5\%) gegeben, wobei der pH-Wert bei 4 lag. Die wäßrige Phase wurde mit $\mathrm{CH}_{2} \mathrm{Cl}_{2}(8 \times 10 \mathrm{~mL})$ extrahiert. Die vereinigten organischen Phasen wurden über $\mathrm{MgSO}_{4}$ getrocknet und filtriert. Nach Entfernung des Lösungsmittels wurde der Rückstand durch Säulenchromatographie auf Kieselgel $\left(\mathrm{CH}_{2} \mathrm{Cl}_{2} / \mathrm{MeOH}=30: 1\right)$ gereinigt. Man erhielt $( \pm)$-Camptothecin 11 (2.8 mg, $8.0 \mu \mathrm{mol}, 42 \%)$ als gebrochen-weißen Feststoff.

$\mathbf{R}_{\mathbf{f}}=0.18\left(\mathrm{CH}_{2} \mathrm{Cl}_{2} / \mathrm{MeOH}=30: 1\right)$.

UV $\left(\mathrm{CH}_{3} \mathrm{CN}\right): \lambda_{\max }(\lg \varepsilon)=194.5 \mathrm{~nm}$ (4.454), 218.5 (4.527), 252.5 (4.434), 288.5 (3.767), 362.5 (4.243), 377.5 (4.174).

IR (KBr): $\widetilde{v}=3277 \mathrm{~cm}^{-1}(\mathrm{O}-\mathrm{H}), 2925(\mathrm{C}-\mathrm{H}), 1755,1651,(\mathrm{C}=\mathrm{O}), 1583,1500,1460\left(\mathrm{CH}_{2}\right)$, 1158, 1100, 1039 (C-O), 788, 767, $724\left(\mathrm{CH}_{2}\right)$.

${ }^{1}$ H-NMR $\left(300 \mathrm{MHz}, \mathrm{CDCl}_{3}\right): \delta=1.04\left(\mathrm{t},{ }^{3} \mathrm{~J}=7.2 \mathrm{~Hz}, 3 \mathrm{H}, \mathrm{CH}_{3}\right), 1.90\left(\mathrm{~m}_{\mathrm{c}}, 2 \mathrm{H}, \mathrm{CH}_{2} \mathrm{CH}_{3}\right)$, 5.28-5.35 (m, 3 H, 5-H, 17- $\left.\mathrm{H}_{\mathrm{a}}\right), 5.78\left(\mathrm{~d},{ }^{2} \mathrm{~J}=16.2 \mathrm{~Hz}, 1 \mathrm{H}, 17-\mathrm{H}_{\mathrm{b}}\right), 7.63-7.70(\mathrm{~m}, 2 \mathrm{H}, 10-\mathrm{H}$, 14-H), $7.83\left(\mathrm{~m}_{\mathrm{c}}, 1 \mathrm{H}, 11-\mathrm{H}\right), 7.94\left(\mathrm{~d},{ }^{3} J=8.2 \mathrm{~Hz}, 1 \mathrm{H}, 9-\mathrm{H}\right), 8.24\left(\mathrm{~d},{ }^{3} J=8.6 \mathrm{~Hz}, 1 \mathrm{H}, 12-\mathrm{H}\right)$, 8.39 (s, $1 \mathrm{H}, 7-\mathrm{H})$.

MS (ESI): $m / z(\%)=349.1(100)[\mathrm{M}+\mathrm{H}]^{+}$.

$\mathrm{C}_{20} \mathrm{H}_{16} \mathrm{~N}_{2} \mathrm{O}_{4}(348.35)$

Ber.: $\quad 349.1182 \quad[\mathrm{M}+\mathrm{H}]^{+}$

Gef.: $\quad 349.1183$ (ESI-HRMS) 


\section{Methanolyse-modifizierte Domino-Knoevenagel-hetero-Diels-}

\section{Alder-Reaktion und Aufbau des Lactonrings}

\subsection{4-(2-Benzyloxycarbonyl-2,3-dihydro-1H-pyrrolo[3,4-b]chinolin-3-} ylmethyl)-6-ethoxy-5-ethyl-3-methyloxycarbonyl-tetrahydropyran-2on (146)

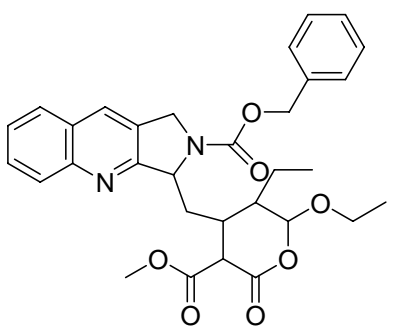

Zu einer Suspension von 96 (262 mg, 0.756 mmol, 1.00 Äq.) und EDDA (6 mg) in Methanol (7 mL) in einem Druckkolben wurden Meldrum-Säure (109 mg, 0.756 mmol, 1.00 Äq.) und eine Lösung des Enolethers 64 (227 mg, 2.27 mmol, 3.00 Äq.) in Methanol (0.5 mL) gegeben. Anschließend wurde das Reaktionsgemisch bei $55^{\circ} \mathrm{C}$ für $3 \mathrm{~h}$ gerührt. Nach Abkühlung auf RT wurde die Reaktionsmischung im Vakuum konzentriert. Der Rückstand wurde durch Säulenchromatographie auf Kieselgel $($ Pentan/EtOAc $=2.5: 1)$ gereinigt. Man erhielt 146 (316 mg, $0.578 \mathrm{mmol}, 76 \%$ ) als weißen Feststoff.

$\mathbf{R}_{\mathbf{f}}=0.31-0.46($ Petan $/$ EtOAc $=2: 1)$.

MS (ESI): $m / z(\%)=547.2(100)[\mathrm{M}+\mathrm{H}]^{+}$.

$\mathbf{C}_{31} \mathbf{H}_{34} \mathbf{N}_{2} \mathbf{O}_{7}(546.61)$

Ber.: $\quad 547.2438 \quad[\mathrm{M}+\mathrm{H}]^{+}$

Gef.: $\quad 547.2437$ (ESI-HRMS) 
$6.24-(2-2,2,2-T r i c h l o r-e t h o x y c a r b o n y l-2,3-d i h y d r o-1 H$-pyrrolo[3,4b]chinolin-3-ylmethyl)-6-ethoxy-5-ethyl-3-methyloxycarbonyltetrahydropyran-2-on (126)

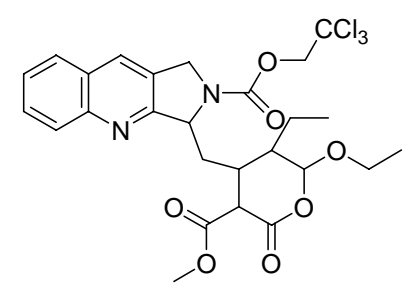

Eine Mischung von 114 (98 mg, 0.25 mmol, 1.0 Äq.), Meldrum-Säure (39 mg, 0.27 mmol, 1.1 Äq.), EDDA (4 mg), 64 (0.10 g, 1.0 mmol, 4.0 Äq.), Methylorthoacetat $(63 \mu \mathrm{L}$, $0.50 \mathrm{mmol}, 2.0$ Äq. $)$ in Methanol $(2.5 \mathrm{~mL})$ wurde bei $45^{\circ} \mathrm{C}$ für $2 \mathrm{~h}$ gerührt. Anschließend wurde das Lösungsmittel unter vermindertem Druck entfernt. Der Rückstand wurde durch Säulenchromatographie auf Kieselgel $($ Pentan/EtOAc $=2.5: 1)$ gereinigt. Man erhielt 126 (126 mg, $0.214 \mathrm{mmol}, 85 \%$ ) als weißen Feststoff.

$\mathbf{R}_{\mathbf{f}}=0.33-0.52(\mathrm{Petan} / \mathrm{EtOAc}=2: 1)$.

MS (ESI): $m / z(\%)=589.1(96), 587.1(100)[\mathrm{M}+\mathrm{H}]^{+}$.

$\mathrm{C}_{26} \mathbf{H}_{29} \mathrm{Cl}_{3} \mathbf{N}_{2} \mathbf{O}_{7}(587.88)$

Ber.:

$587.1113,589.1083$

$[\mathrm{M}+\mathrm{H}]^{+}$

609.0932, $611.0903 \quad[\mathrm{M}+\mathrm{Na}]^{+}$

Gef.: $\quad 587.1112,589.1082$ (ESI-HRMS)

$609.0931,611.0902$ 


\section{$6.32-(9-0 x 0-8-m e t h y l o x y c a r b o n y l-5 b, 6,7,8,9,11-h e x a h y d r o-$ indolizino[1,2-b]-chinolin-7-yl)-butyraldehyd (62)}

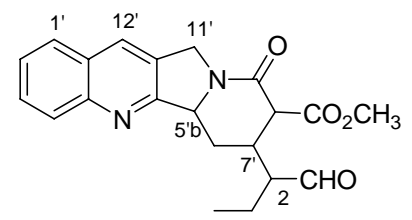

\section{Ausgehend von 146}

Eine Suspension von 146 (316 mg, 0.578 mmol, 1.00 Äq.) und Palladium auf Aktivkohle $(10 \% \mathrm{Pd}, 62 \mathrm{mg})$ in Ethanol $(25 \mathrm{~mL})$ wurde bei $\mathrm{RT}$ unter $\mathrm{H}_{2}$-Atmosphäre $\left(\mathrm{H}_{2}\right.$-Ballon) für $2 \mathrm{~h}$ gerührt. Dann wurde die Reaktionsmischung über Celite ${ }^{\circledR}$ filtriert und die Mutterlauge im Vakuum konzentriert. Der Rückstand wurde durch Säulenchromatographie auf Kieselgel $($ Pentan $/$ EtOAc $=1: 2)$ gereinigt. Man erhielt den Aldehyd $62(152 \mathrm{mg}, 0.415 \mathrm{mmol}, 72 \%)$ als weißen Feststoff.

\section{Ausgehend von 126}

Eine Mischung von 126 (80 mg, 0.14 mmol, 1.0 Äq.), aktiviertem Zn-Pulver ${ }^{72}$ (150 mg), Essigsäure (40 mg, $0.67 \mathrm{mmol}, 4.9$ Äq.) in THF ( $8 \mathrm{~mL}$ ) wurde bei RT für $30 \mathrm{~min}$ gerührt. Anschließend wurde Wasser $(0.10 \mathrm{~mL})$ hingefügt und für $30 \mathrm{~min}$ gerührt. Dann wurden Wasser $(0.10 \mathrm{~mL})$ und zwei Tropfen Essigsäure zugegeben und weiter bei RT für $30 \mathrm{~min}$ gerührt. Nach Zugabe von $\mathrm{K}_{2} \mathrm{CO}_{3}(0.20 \mathrm{~g})$ wurde die Reaktionmischung über Celite ${ }^{\circledR}$ filtriert und die Mutterlauge im Vakuum konzentriert. Der Rückstand wurde durch Säulenchromatographie auf Kieselgel $($ Petan/EtOAc $=1: 2)$ gereinigt. Man erhielt den Aldehyd $62(32 \mathrm{mg}$, $0.087 \mathrm{mmol}, 64 \%$ ) als weißen Feststoff.

$\mathbf{R}_{\mathbf{f}}=0.24-0.29($ Petan $/$ EtOAc $=1: 2)$.

${ }^{1}$ H-NMR (300 MHz, $\mathrm{CDCl}_{3}$, ausgewählt): $\delta=0.99-1.10\left(\mathrm{~m}, 3 \mathrm{H}, \mathrm{CH}_{2} \mathrm{CH}_{3}\right), 3.80-3.85$ (m, $\left.3 \mathrm{H}, \mathrm{CO}_{2} \mathrm{CH}_{3}\right), 4.65-4.80\left(\mathrm{~m}, 1 \mathrm{H}, 11^{\prime}-\mathrm{H}_{\mathrm{a}}\right), 4.87-5.35\left(\mathrm{~m}, 2 \mathrm{H}, 11^{\prime}-\mathrm{H}_{\mathrm{b}}, 7^{\prime}-\mathrm{H}\right), 7.57\left(\mathrm{~m}_{\mathrm{c}}, 1 \mathrm{H}\right.$, 2'-H), 7.73 ( $\left.\mathrm{m}_{\mathrm{c}}, 1 \mathrm{H}, 3^{\prime}-\mathrm{H}\right), 7.84$ (d, ${ }^{3} \mathrm{~J}=8.1 \mathrm{~Hz}, 1 \mathrm{H}, 1$ '-H), 8.03-8.11 (m, 2 H, 4'-H, 12'-H), 9.66-9.79 (m, $1 \mathrm{H}, \mathrm{CHO})$.

MS (ESI): $m / z(\%)=367.1(100)[\mathrm{M}+\mathrm{H}]^{+}$.

$\mathbf{C}_{21} \mathbf{H}_{22} \mathbf{N}_{2} \mathbf{O}_{4}(366.41)$

Ber.: $\quad 367.1652 \quad[\mathrm{M}+\mathrm{H}]^{+}$

Gef.: $\quad 367.1652$ (ESI-HRMS) 


\subsection{2-(9-Oxo-8-methyloxycarbonyl-5b,6,7,8,9,11-hexahydro- indolizino[1,2-b]-chinolin-7-yl)-buttersäure (147)}

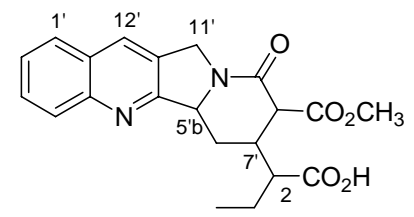

Zu einer Mischung von 62 (151 mg, 0.412 mmol, 1.00 Äq.), 2-Methyl-2-buten (3.50 mL) in tert-BuOH $(8 \mathrm{~mL})$ und Wasser $(2 \mathrm{~mL})$ bei RT wurde $\mathrm{NaH}_{2} \mathrm{PO}_{4} \cdot \mathrm{H}_{2} \mathrm{O}(569 \mathrm{mg}, 4.12 \mathrm{mmol}$, 10.0 Äq.) und $\mathrm{NaClO}_{2}$ (80\% tech., $466 \mathrm{mg}, 4.12 \mathrm{mmol}, 10.0$ Äq.) gegeben. Nach $25 \mathrm{~min}$ Rühren wurden $5 \mathrm{~mL}$ einer Pufferlösung $(\mathrm{pH}=7)$ hingefügt. Die wäßrige Phase wurde mit EtOAc $(3 \times 30 \mathrm{~mL})$ extrahiert. Die vereinigten organischen Phasen wurden mit Wasser (40 mL) und gesättiger NaCl-Lösung gewaschen, über $\mathrm{MgSO}_{4}$ getrocknet und filtriert. Nach Entfernung des Lösungsmittels erhielt man die Carbonsäure 147 (157 mg, 0.412 mmol, 100\%) als weißen Feststoff.

MS (ESI): $m / z(\%)=383.1(100)[\mathrm{M}+\mathrm{H}]^{+}$.

$\mathbf{C}_{21} \mathbf{H}_{22} \mathbf{N}_{2} \mathbf{O}_{5}(382.41)$

Ber.: $\quad 383.1601 \quad[\mathrm{M}+\mathrm{H}]^{+}$

Gef.: $\quad 383.1600$ (ESI-HRMS)

\subsection{2-(9-Oxo-8-methyloxycarbonyl-5b,6,7,8,9,11-hexahydro- indolizino[1,2-b]-chinolin-7-yl)-buttersäuremethylester (148)}

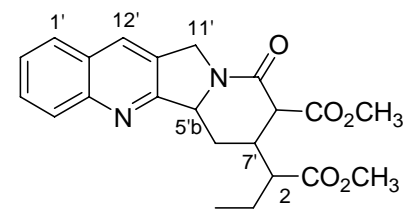

$\mathrm{Zu}$ einer Suspension von 147 (14 mg, $44 \mu \mathrm{mmol}, 1.0$ Äq.) in Methanol (0.4 mL) und Toluol (1.4 mL) bei RT wurde TMS-CHN 2 (2.0 M in $\mathrm{Et}_{2} \mathrm{O}, 26 \mu \mathrm{L}, 53 \mu \mathrm{mol}, 1.2 \mathrm{Äq}$.) gegeben. Nach $1 \mathrm{~h}$ Rühren wurde die Reaktionsmischung im Vakuum konzentriert. Der Rückstand wurde 
durch Säulenchromatographie auf Kieselgel (Pentan/EtOAc $=1: 2)$ gereinigt. Man erhielt 148 (11 mg, $33 \mu \mathrm{mol}, 76 \%$ ) als weißen Feststoff.

$\mathbf{R}_{\mathbf{f}}=0.33-0.36(\operatorname{Petan} /$ EtOAc $=1: 2)$.

MS (ESI): $m / z(\%)=397.1(100)[\mathrm{M}+\mathrm{H}]^{+}$.

$\mathbf{C}_{22} \mathbf{H}_{24} \mathbf{N}_{2} \mathbf{O}_{5}$ (396.44)

$\begin{array}{lll}\text { Ber.: } & 397.1758 & {[\mathrm{M}+\mathrm{H}]^{+}} \\ & 419.1577 & {[\mathrm{M}+\mathrm{Na}]^{+}} \\ \text {Gef.: } & 397.1758 & (\text { ESI-HRMS }) \\ & 419.1577 & \end{array}$

\subsection{2-(8-Methyloxycarbonyl-9-oxo-9,11-dihydro-indolizino[1,2-}

b]chinolin-7-yl)-buttersäuremethylester (157)

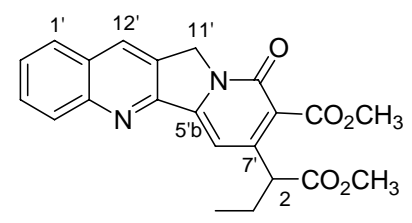

Eine Mischung von 148 (10 mg, $25 \mu \mathrm{mol}, 1.0$ Äq.) und DDQ (13 mg, $55 \mu \mathrm{mol}, 2.2$ Äq.) in 1,4-Dioxan $(0.6 \mathrm{~mL})$ im Druckkolben wurde bei $60^{\circ} \mathrm{C}$ für $70 \mathrm{~min}$ gerührt, wobei sich Niederschlag bildete. Nach Abkühlung auf RT wurde der Niederschlag abfiltriert und mit $\mathrm{CH}_{2} \mathrm{Cl}_{2}$ gewaschen. Die Mutterlauge wurde im Vakuum konzentriert und der Rückstand durch Säulenchromatographie auf Kieselgel $\left(\right.$ EtOAc/ $\left.\mathrm{NEt}_{3}=40: 1\right)$ gereinigt. Man erhielt 157 (5.0 mg, $13 \mu \mathrm{mol}, 51 \%$ ) als hellgelben Feststoff.

$\mathbf{R}_{\mathbf{f}}=0.40\left(\mathrm{EtOAc} / \mathrm{NEt}_{3}=20: 1\right)$

UV $\left(\mathrm{CH}_{3} \mathrm{CN}\right): \lambda_{\max }(\lg \varepsilon)=194.5 \mathrm{~nm}$ (4.531), 217.5 (4.614), 253.0 (4.516), 288.0 (3.828), 365.0 (4.331), 378.0 (4.277).

IR (KBr): $\widetilde{v}=2953 \mathrm{~cm}^{-1}(\mathrm{C}-\mathrm{H}), 1734,1653,1606(\mathrm{C}=\mathrm{O}), 1531,1433,1403\left(\mathrm{CH}_{3}\right), 1169$, $1128(\mathrm{C}-\mathrm{O}), 785,758,725\left(\mathrm{CH}_{3}\right)$.

${ }^{1} \mathbf{H}-\mathrm{NMR}\left(300 \mathrm{MHz}, \mathrm{CDCl}_{3}\right): \delta=0.97\left(\mathrm{t},{ }^{3} \mathrm{~J}=7.7 \mathrm{~Hz}, 3 \mathrm{H}, \mathrm{CH}_{2} \mathrm{CH}_{3}\right), 1.87-2.05(\mathrm{~m}, 1 \mathrm{H}$, 3- $\left.\mathrm{H}_{\mathrm{a}}\right), 2.15-2.32\left(\mathrm{~m}, 1 \mathrm{H}, 3-\mathrm{H}_{\mathrm{b}}\right), 3.72\left(\mathrm{~s}, 3 \mathrm{H}, \mathrm{CO}_{2} \mathrm{CH}_{3}\right), 3.77\left(\mathrm{t},{ }^{3} \mathrm{~J}=7.7 \mathrm{~Hz}, 1 \mathrm{H}, 2-\mathrm{H}\right), 3.99$ 
(s, $3 \mathrm{H}$, Pyridon- $\mathrm{CO}_{2} \mathrm{CH}_{3}$ ), 5.25 (s, 2 H, 11'-H), 7.40 (s, 1 H, 6'-H), 7.65 (m, 1 H, 2'-H), 7.82 $\left(\mathrm{m}_{\mathrm{c}}, 1 \mathrm{H}, 3^{\prime}-\mathrm{H}\right), 7.92\left(\mathrm{~d},{ }^{3} \mathrm{~J}=8.2 \mathrm{~Hz}, 1 \mathrm{H}, 1^{\prime}-\mathrm{H}\right), 8.22$ (d, $\left.{ }^{3} \mathrm{~J}=8.6 \mathrm{~Hz}, 1 \mathrm{H}, 4^{\prime}-\mathrm{H}\right), 8.37$ (s, $1 \mathrm{H}$, $\left.12^{\prime}-\mathrm{H}\right)$.

MS (ESI): $m / z(\%)=415.1(100)[\mathrm{M}+\mathrm{Na}]^{+}$.

$\mathbf{C}_{22} \mathbf{H}_{20} \mathbf{N}_{2} \mathbf{O}_{5}(392.40)$

$\begin{array}{lll}\text { Ber.: } & 393.1445 & {[\mathrm{M}+\mathrm{H}]^{+}} \\ & 415.1264 & {[\mathrm{M}+\mathrm{Na}]^{+}} \\ & 431.1003 & {[\mathrm{M}+\mathrm{K}]^{+}} \\ \text {Gef.: } & 393.1444 & (\mathrm{ESI}-\mathrm{HRMS}) \\ & 415.1263 & \end{array}$

431.1003

\subsection{2-(8-Formyl-9-oxo-9,11-dihydro-indolizino[1,2-b]chinolin-7-yl)- buttersäure-methylester (158)}

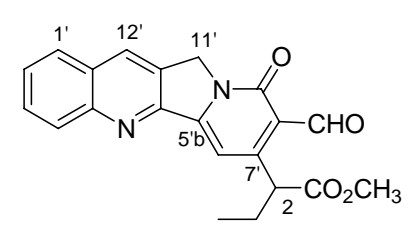

$\mathrm{Zu}$ einer Lösung von $157\left(8.4 \mathrm{mg}, 21 \mu \mathrm{mol}, 1.0\right.$ Äq.) in Dichlormethan $(2 \mathrm{~mL})$ bei $-78^{\circ} \mathrm{C}$ wurde DIBAL-H (1.0 M in Dichlormethan, $20 \mu \mathrm{L}, 20 \mu \mathrm{mol}, 0.95$ Äq.) tropfenweise gegeben. Die Reaktionsmischung wurde für 50 min bei dieser Temperatur gerührt. Anschließend wurde eine verdünnte Lösung von DIBAL-H (1.0 M in Dichlormethan, $26 \mu \mathrm{L}, 26 \mu \mathrm{mol}, 1.2$ Äq.) in Dichlormethan $(0.2 \mathrm{~mL})$ tropfenweise gegeben. Nach $1 \mathrm{~h}$ Rühren bei $-78^{\circ} \mathrm{C}$ wurde DIBAL-H (1.0 M in Dichlormethan, $26 \mu \mathrm{L}, 26 \mu \mathrm{mol}, 1.2$ Äq.) zugetropft und weiter für $50 \mathrm{~min}$ bei dieser Temperatur gerührt. Durch Zugabe von Methanol $(0.1 \mathrm{~mL})$ und Wasser $(4 \mathrm{~mL})$ wurde die Reaktion beendet. Die Mischung wurde dann auf RT erwärmt und die wäßrige Phase mit $\mathrm{CH}_{2} \mathrm{Cl}_{2}(3 \times 10 \mathrm{~mL})$ extrahiert. Die vereinigten organischen Phasen wurden über $\mathrm{Na}_{2} \mathrm{SO}_{4}$ getrocknet und filtriert. Nach Entfernung des Lösungsmittels wurde der Rückstand durch Säulenchromatographie auf Kieselgel (EtOAc/Methanol=20:1) gereinigt. Man erhielt den Aldehyd 158 (0.40 mg, $1.1 \mu \mathrm{mol}, 5 \%)$ als gelben Feststoff. 
$\mathbf{R}_{\mathbf{f}}=0.59($ EtOAc/Methanol $=20: 1)$.

${ }^{1}$ H-NMR $\left(300 \mathrm{MHz}, \mathrm{CDCl}_{3}\right): \delta=0.99\left(\mathrm{t},{ }^{3} \mathrm{~J}=7.6 \mathrm{~Hz}, 3 \mathrm{H}, \mathrm{CH}_{2} \mathrm{CH}_{3}\right), 1.80-1.98(\mathrm{~m}, 1 \mathrm{H}$, 3- $\left.\mathrm{H}_{\mathrm{a}}\right), 2.17-2.34\left(\mathrm{~m}, 1 \mathrm{H}, 3-\mathrm{H}_{\mathrm{b}}\right), 3.71\left(\mathrm{~s}, 3 \mathrm{H}, \mathrm{CO}_{2} \mathrm{CH}_{3}\right), 5.22\left(\mathrm{t},{ }^{3} \mathrm{~J}=7.5 \mathrm{~Hz}, 1 \mathrm{H}, 2-\mathrm{H}\right), 5.34$ (s, $\left.2 \mathrm{H}, 11^{\prime}-\mathrm{H}\right), 7.41$ (s, $1 \mathrm{H}, 6$ '’-H), 7.70 (m $\left.\mathrm{m}_{\mathrm{c}}, 1 \mathrm{H}, 2^{\prime}-\mathrm{H}\right), 7.86\left(\mathrm{~m}_{\mathrm{c}}, 1 \mathrm{H}, 3^{\prime}-\mathrm{H}\right), 7.97$ (d, 3 J = $\left.8.1 \mathrm{~Hz}, 1 \mathrm{H}, 1^{\prime}-\mathrm{H}\right), 8.26$ (d, ${ }^{3} \mathrm{~J}=8.7 \mathrm{~Hz}, 1 \mathrm{H}, 4^{\prime}$-H), 8.44 (s, $\left.1 \mathrm{H}, 12^{\prime}-\mathrm{H}\right) 10.67$ (s, $\left.1 \mathrm{H}, \mathrm{CHO}\right)$. MS (ESI): $m / z(\%)=363.1(100)[\mathrm{M}+\mathrm{H}]^{+}$.

$\mathrm{C}_{21} \mathrm{H}_{18} \mathbf{N}_{2} \mathbf{O}_{4}(362.38)$

$\begin{array}{lll}\text { Ber.: } & 363.1339 & {[\mathrm{M}+\mathrm{H}]^{+}} \\ & 385.1158 & {[\mathrm{M}+\mathrm{Na}]^{+}} \\ \text {Gef.: } & 363.1341 & (\mathrm{ESI}-\mathrm{HRMS}) \\ & 385.1159 & \end{array}$

385.1159

\section{$6.8 \quad 2-(8-I s o b u t y l o x y c a r b o n y l-9-0 x 0-9,11-d i h y d r o-i n d o l i z i n o[1,2-$ b]chinolin-7-yl)-buttersäuremethylester (160)}

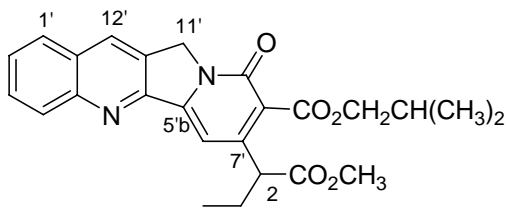

$\mathrm{Zu}$ einer Lösung von DIBAL-H (1.0 M in Dichlormethan, $45 \mu \mathrm{L}, 45 \mu \mathrm{mol}, 2.1 \mathrm{Äq})$ in Dichlormethan $(1.0 \mathrm{~mL})$ bei $-78^{\circ} \mathrm{C}$ wurde eine Lösung von 157 (8.4 mg, $21 \mu \mathrm{mol}, 1.0$ Äq.) in Dichlormethan $(1 \mathrm{~mL})$ gegeben. Nach 85 min Rühren bei dieser Temperatur wurde die Reaktionsmischung nachfolgend bei $0{ }^{\circ} \mathrm{C}$ und RT jeweils für $1 \mathrm{~h}$ gerührt. Durch Zugabe von wässriger 1\%-HCl-Lösung ( $3 \mathrm{~mL})$ wurde die Reaktion beendet. Die wäßrige Phase wurde mit $\mathrm{CH}_{2} \mathrm{Cl}_{2}(3 \times 10 \mathrm{~mL})$ extrahiert. Die vereinigten organischen Phasen wurden über $\mathrm{Na}_{2} \mathrm{SO}_{4}$ getrocknet und filtriert. Nach Entfernung des Lösungsmittels wurde der Rückstand durch Säulenchromatographie auf Kieselgel $\left(\mathrm{CH}_{2} \mathrm{Cl}_{2} / \mathrm{EtOAc}=1: 2\right)$ gereinigt. Man erhielt zwei Fraktionen bzw. 160 (6.0 mg, $14 \mu \mathrm{mol}, 66 \%)$ als hellgelben Feststoff und das Edukt 157 (2.6 mg, $6.6 \mu \mathrm{mol}, 31 \%$ ) als hellgelben Feststoff. 
$\mathbf{R}_{\mathbf{f}}=0.53\left(\mathrm{CH}_{2} \mathrm{Cl}_{2} / \mathrm{EtOAc}=1: 3\right)$.

UV $\left(\mathrm{CH}_{3} \mathrm{CN}\right): \lambda_{\max }(\lg \varepsilon)=195.5 \mathrm{~nm}$ (4.487), 217.5 (4.592), 253.0 (4.497), 288.0 (3.838), 365.0 (4.319), 378.5 (4.261).

IR (KBr): $\widetilde{v}=2962 \mathrm{~cm}^{-1}(\mathrm{C}-\mathrm{H}), 1737,1657,1611(\mathrm{C}=\mathrm{O}), 1535,1458,1405\left(\mathrm{CH}_{3}\right), 1170$, 1126 (C-O), 786, 757, $725\left(\mathrm{CH}_{3}\right)$.

${ }^{1} \mathbf{H}-\mathbf{N M R}\left(300 \mathrm{MHz}, \mathrm{CDCl}_{3}\right): \delta=0.97\left(\mathrm{t},{ }^{3} J=7.2 \mathrm{~Hz}, 3 \mathrm{H}, \mathrm{CH}_{2} \mathrm{CH}_{3}\right), 1.04\left(\mathrm{~d},{ }^{3} J=6.8 \mathrm{~Hz}\right.$, $\left.6 \mathrm{H}, \mathrm{CH}\left(\mathrm{CH}_{3}\right)_{2}\right), 1.87-2.03\left(\mathrm{~m}, 1 \mathrm{H}, 3-\mathrm{H}_{\mathrm{a}}\right), 2.03-2.32\left(\mathrm{~m}, 2 \mathrm{H}, 3-\mathrm{H}_{\mathrm{b}}, \mathrm{CH}\left(\mathrm{CH}_{3}\right)_{2}\right), 3.63-3.84$ (m, $\left.4 \mathrm{H}, \mathrm{CO}_{2} \mathrm{CH}_{3}, 2-\mathrm{H}\right), 4.21\left(\mathrm{~m}_{\mathrm{c}}, 2 \mathrm{H}, \mathrm{CO}_{2} \mathrm{CH}_{2}\right), 5.26$ (s, $\left.2 \mathrm{H}, 11^{\prime}-\mathrm{H}\right), 7.40$ (s, $1 \mathrm{H}, 6$ ' $\left.-\mathrm{H}\right)$, $7.66\left(\mathrm{~m}_{\mathrm{c}}, 1 \mathrm{H}, 2^{\prime}-\mathrm{H}\right), 7.83\left(\mathrm{~m}_{\mathrm{c}}, 1 \mathrm{H}, 3^{\prime}-\mathrm{H}\right), 7.92\left(\mathrm{~d},{ }^{3} J=8.1 \mathrm{~Hz}, 1 \mathrm{H}, 1^{\prime}-\mathrm{H}\right), 8.22\left(\mathrm{~d},{ }^{3} J=8.6\right.$ $\mathrm{Hz}, 1 \mathrm{H}, 4$ '-H), 8.37 (s, $1 \mathrm{H}, 12$ '-H).

MS (ESI): $m / z(\%)=435.1(100)[\mathrm{M}+\mathrm{H}]^{+}$.

$\mathbf{C}_{25} \mathbf{H}_{26} \mathbf{N}_{2} \mathbf{O}_{5}(434.48)$

$\begin{array}{lll}\text { Ber.: } & 435.1914 & {[\mathrm{M}+\mathrm{H}]^{+}} \\ & 457.1732 & {[\mathrm{M}+\mathrm{Na}]^{+}} \\ \text {Gef.: } & 435.1915 & \text { (ESI-HRMS) } \\ & 457.1734 & \end{array}$

\subsection{8-Methyloxycarbonyl-7-(1-dimethoxymethyl-propyl)-5b,7,8,11- tetrahydro-6H-indolizino[1,2-b]chinolin-9-on (151)}

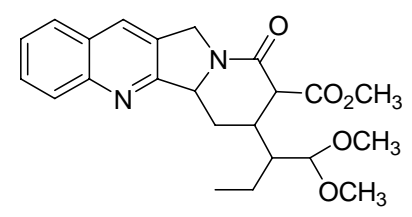

Eine Mischung von 62 (10 mg, $27 \mu \mathrm{mol}, 1.0$ Äq.), $\mathrm{RuCl}_{3} \cdot \mathrm{xH}_{2} \mathrm{O}$ (eine Spatelspitze), Methylorthoformat (3.5 mg, $33 \mu \mathrm{mol}, 1.2$ Äq.) in Methanol (1 mL) im Druckkolben wurde bei $60{ }^{\circ} \mathrm{C}$ für $3.5 \mathrm{~h}$ gerührt. Durch Zugabe von wässriger $5 \%-\mathrm{NaHCO}_{3}$-Lösung $(5 \mathrm{~mL})$ wurde die Reaktion beendet. Die wäßrige Phase wurde mit $\mathrm{CH}_{2} \mathrm{Cl}_{2}(3 \times 10 \mathrm{~mL})$ extrahiert. Die vereinigten organischen Phasen wurden über $\mathrm{Na}_{2} \mathrm{SO}_{4}$ getrocknet und filtriert. Nach Entfernung des Lösungsmittels wurde der Rückstand durch Säulenchromatographie auf Kieselgel (Pentan/EtOAc = 1:1) gereinigt. Man erhielt das Acetal 151 (6.0 mg, $15 \mu \mathrm{mol}$, 54\%) als weißen Feststoff. 
$\mathbf{R}_{\mathbf{f}}=0.24-0.28($ Petan $/$ EtOAc $=1: 1)$.

$\mathrm{C}_{23} \mathrm{H}_{28} \mathbf{N}_{2} \mathbf{O}_{5}$ (412.48).

\subsection{8-Methyloxycarbonyl-7-(1-dimethoxymethyl-propyl)-11H- indolizino[1,2-b]-chinolin-9-on (152)}

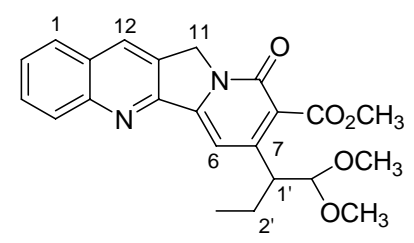

Eine Mischung von 151 (14 mg, $34 \mu \mathrm{mol}, 1.0$ Äq.) und DDQ (15 mg, $68 \mu \mathrm{mol}, 2.0$ Äq.) in 1,4-Dioxan $(2 \mathrm{~mL})$ wurde bei $65^{\circ} \mathrm{C}$ für $1 \mathrm{~h}$ gerührt, wobei sich Niederschlag bildete. Nach Abkühlung auf RT wurde der Niederschlag abfiltriert und mit $\mathrm{CH}_{2} \mathrm{Cl}_{2}$ gewaschen. Die Mutterlauge wurde im Vakuum konzentriert und der Rückstand durch Säulenchromatographie auf Kieselgel (EtOAc/ $\left.\mathrm{NEt}_{3}=20: 1\right)$ gereinigt. Man erhielt $152(11 \mathrm{mg}, 27 \mu \mathrm{mol}, 79 \%)$ als hellgelben Feststoff.

$\mathbf{R}_{\mathbf{f}}=0.38\left(\mathrm{EtOAc} / \mathrm{NEt}_{3}=20: 1\right)$.

${ }^{1}$ H-NMR $\left(300 \mathrm{MHz}, \mathrm{CDCl}_{3}\right): \delta=0.87\left(\mathrm{t},{ }^{3} \mathrm{~J}=7.4 \mathrm{~Hz}, 3 \mathrm{H}, \mathrm{CH}_{2} \mathrm{CH}_{3}\right), 1.70-1.88(\mathrm{~m}, 1 \mathrm{H}$, 2'- $\left.\mathrm{H}_{\mathrm{a}}\right), 1.88-2.06\left(\mathrm{~m}, 1 \mathrm{H}, 2^{\prime}-\mathrm{H}_{\mathrm{b}}\right), 3.04-3.16$ (m, $1 \mathrm{H}, 1$ '-H), 3.32 (s, $\left.3 \mathrm{H}, \mathrm{OCH}_{3}\right), 3.41$ (s, $\left.3 \mathrm{H}, \mathrm{OCH}_{3}\right), 3.96\left(\mathrm{~s}, 3 \mathrm{H}, \mathrm{CO}_{2} \mathrm{CH}_{3}\right), 4.58\left(\mathrm{~d},{ }^{3} \mathrm{~J}=6.5 \mathrm{~Hz}, 1 \mathrm{H}, \mathrm{CH}\left(\mathrm{OCH}_{3}\right)_{2}\right), 5.28(\mathrm{~s}, 2 \mathrm{H}$, 11-H), 7.34 (s, $1 \mathrm{H}, 6-\mathrm{H}), 7.66\left(\mathrm{~m}_{\mathrm{c}}, 1 \mathrm{H}, 2-\mathrm{H}\right), 7.83\left(\mathrm{~m}_{\mathrm{c}}, 1 \mathrm{H}, 3-\mathrm{H}\right), 7.93\left(\mathrm{~d},{ }^{3} J=8.1 \mathrm{~Hz}, 1 \mathrm{H}\right.$, $1-\mathrm{H}), 8.23\left(\mathrm{~d},{ }^{3} \mathrm{~J}=8.5 \mathrm{~Hz}, 1 \mathrm{H}, 4-\mathrm{H}\right), 8.38$ (s, $\left.1 \mathrm{H}, 12-\mathrm{H}\right)$.

MS (ESI): $m / z(\%)=431.1(100)[\mathrm{M}+\mathrm{Na}]^{+}$.

$\mathrm{C}_{23} \mathbf{H}_{24} \mathbf{N}_{2} \mathbf{O}_{5}(408.45)$

Ber.:

$$
\begin{array}{ll}
409.1758 & {[\mathrm{M}+\mathrm{H}]^{+}} \\
431.1577 & {[\mathrm{M}+\mathrm{Na}]^{+}} \\
447.1316 & {[\mathrm{M}+\mathrm{K}]^{+}}
\end{array}
$$


Gef.:

409.1757 (ESI-HRMS)

431.1576

447.1316

\subsection{8-Hydroxymethyl-7-(1-dimethoxymethyl-propyl)-11H-indolizino[1,2- b]-chinolin-9-on (154)}

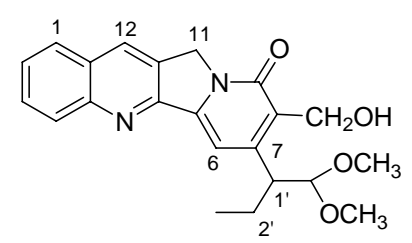

Zu einer Lösung von $152\left(11 \mathrm{mg}, 27 \mu \mathrm{mol}, 1.0\right.$ Äq.) in Dichlormethan $(1.5 \mathrm{~mL})$ bei $-78^{\circ} \mathrm{C}$ wurde DIBAL-H (1.0 M in DCM, $54 \mu \mathrm{L}, 54 \mu \mathrm{mol}, 2.0$ Äq.) tropfenweise gegeben. Es wurde bei dieser Temperatur für $1.5 \mathrm{~h}$ gerührt. Anschließend wurden Methanol (2 mL) und $\mathrm{NaBH}_{4}$ (12 mg, 0.32 mmol, 12 Äq.) gegeben. Dann wurde das Reaktionsgemisch auf RT erwärmt und für 40 min gerührt. Durch Zugabe von Natronlauge $(1 \mathrm{~N}, 5 \mathrm{~mL})$ wurde die Reaktion beendet. Die wäßrige Phase wurde mit $\mathrm{CH}_{2} \mathrm{Cl}_{2}(3 \times 10 \mathrm{~mL})$ extrahiert. Die vereinigten organischen Phasen wurden über $\mathrm{Na}_{2} \mathrm{SO}_{4}$ getrocknet und filtriert. Nach Entfernung des Lösungsmittels wurde der Rückstand durch Säulenchromatographie auf Kieselgel $($ EtOAc $/ \mathrm{MeOH}=20: 1)$ gereinigt. Man erhielt zwei Fraktionen bzw. den Alkohol 154 (7.3 mg, $19 \mu \mathrm{mol}, 71 \%)$ als gelben Feststoff und das Edukt 152 (0.70 mg, $1.7 \mu \mathrm{mol}, 6 \%)$ als hellgelben Feststoff.

$\mathbf{R}_{\mathbf{f}}=0.20(\mathrm{EtOAc} / \mathrm{MeOH}=30: 1)$.

UV $\left(\mathrm{CH}_{3} \mathrm{CN}\right): \lambda_{\max }(\lg \varepsilon)=197.5 \mathrm{~nm}$ (4.466), 220.5 (4.529), 244.5 (4.419), 252.5 (4.455), 290.0 (3.826), 332.5 (3.916), 367.5 (4.252).

IR (KBr): $\widetilde{v}=3355 \mathrm{~cm}^{-1}(\mathrm{O}-\mathrm{H}), 2961(\mathrm{C}-\mathrm{H}), 1653(\mathrm{C}=\mathrm{O}), 1589,1455,1401\left(\mathrm{CH}_{3}, \mathrm{CH}_{2}\right)$, 1143, 1066 (C-O), 754, $724\left(\mathrm{CH}_{3}, \mathrm{CH}_{2}\right)$. 
${ }^{1} \mathbf{H}-\mathbf{N M R}\left(300 \mathrm{MHz}, \mathrm{CDCl}_{3}\right): \delta=0.84\left(\mathrm{t},{ }^{3} \mathrm{~J}=7.0 \mathrm{~Hz}, 3 \mathrm{H}, \mathrm{CH}_{2} \mathrm{CH}_{3}\right), 1.71-1.91(\mathrm{~m}, 1 \mathrm{H}$, 2'- $\left.\mathrm{H}_{\mathrm{a}}\right), 1.91-2.10\left(\mathrm{~m}, 1 \mathrm{H}, 2^{\prime}-\mathrm{H}_{\mathrm{b}}\right), 3.23-3.38\left(\mathrm{~m}, 4 \mathrm{H}, 1^{\prime}-\mathrm{H}, \mathrm{OCH}_{3}\right), 3.45$ (s, $\left.3 \mathrm{H}, \mathrm{OCH}_{3}\right), 4.00$ $\left(\mathrm{m}_{\mathrm{c}}, 1 \mathrm{H}, \mathrm{OH}\right), 4.58\left(\mathrm{~d},{ }^{3} \mathrm{~J}=7.4 \mathrm{~Hz}, 1 \mathrm{H}, \mathrm{CH}\left(\mathrm{OCH}_{3}\right)_{2}\right), 4.69-4.80\left(\mathrm{~m}, 1 \mathrm{H}, \mathrm{CH}_{\mathrm{a}} \mathrm{H}_{\mathrm{b}} \mathrm{OH}\right)$, 4.89-5.01 (m, $\left.1 \mathrm{H}, \mathrm{CH}_{\mathrm{a}} \mathrm{H}_{\mathrm{b}} \mathrm{OH}\right), 5.27$ (s, $\left.2 \mathrm{H}, 11-\mathrm{H}\right), 7.30$ (s, $\left.1 \mathrm{H}, 6-\mathrm{H}\right), 7.64\left(\mathrm{~m}_{\mathrm{c}}, 1 \mathrm{H}, 2-\mathrm{H}\right)$, $7.81\left(\mathrm{~m}_{\mathrm{c}}, 1 \mathrm{H}, 3-\mathrm{H}\right), 7.92\left(\mathrm{~d},{ }^{3} J=8.1 \mathrm{~Hz}, 1 \mathrm{H}, 1-\mathrm{H}\right), 8.22\left(\mathrm{~d},{ }^{3} J=8.5 \mathrm{~Hz}, 1 \mathrm{H}, 4-\mathrm{H}\right), 8.36$ (s, 1 H, 12-H).

MS (ESI): $m / z(\%)=381.1(100)[\mathrm{M}+\mathrm{H}]^{+}$.

$\mathrm{C}_{22} \mathrm{H}_{24} \mathbf{N}_{2} \mathbf{O}_{4}(380.44)$

Ber.:

$381.1808 \quad[\mathrm{M}+\mathrm{H}]^{+}$

$403.1628 \quad[\mathrm{M}+\mathrm{Na}]^{+}$

Gef.: $\quad 381.1808$ (ESI-HRMS)

403.1628

\subsection{8-Hydroxymethyl-7-(1-methoxymethylen-propyl)-11H-indolizino[1,2- b]-chinolin-9-on (155)}

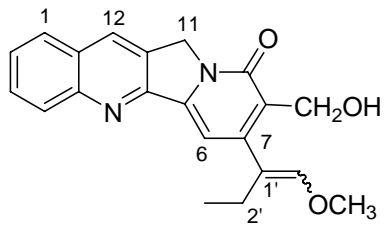

Eine Mischung von 154 (3.2 mg, $8.4 \mu \mathrm{mol}, 1.0$ Äq.) und $\mathrm{LiBF}_{4}$ (6.7 mg, $71 \mu \mathrm{mol}, 8.5$ Äq.) in $\mathrm{CH}_{3} \mathrm{CN} / \mathrm{H}_{2} \mathrm{O}(50: 1,0.3 \mathrm{~mL})$ wurde bei RT für $30 \mathrm{~min}$ und danach bei $65^{\circ} \mathrm{C}$ für $70 \mathrm{~min}$ gerührt. Nach leichter Abkühlung wurden $\mathrm{LiBF}_{4}(5.8 \mathrm{mg}, 62 \mu \mathrm{mol}, 7.4$ Äq.) und ein Mischung von $\mathrm{CH}_{3} \mathrm{CN} / \mathrm{H}_{2} \mathrm{O}(50: 1,0.1 \mathrm{~mL})$ zugegeben. Dann wurde die Reaktionsmischung bei $65{ }^{\circ} \mathrm{C}$ für $30 \mathrm{~min}$ gerührt. Durch Zugabe von wässriger $5 \%-\mathrm{NaHCO}_{3}$-Lösung $(1 \mathrm{~mL})$ wurde die Reaktion beendet. Die wäßrige Phase wurde mit $\mathrm{CH}_{2} \mathrm{Cl}_{2}(3 \times 10 \mathrm{~mL})$ extrahiert. Die vereinigten organischen Phasen wurden über $\mathrm{Na}_{2} \mathrm{SO}_{4}$ getrocknet und filtriert. Nach Entfernung des Lösungsmittels wurde der Rückstand durch Säulenchromatographie auf Kieselgel $\quad\left(\mathrm{EtOAc} / \mathrm{NEt}_{3}=40: 3 \rightarrow \mathrm{EtOAc} / \mathrm{MeOH}=20: 1\right) \quad$ gereinigt. Man erhielt zwei Fraktionen bzw. den Enolether 155 (1.7 mg, $5.0 \mu \mathrm{mol}, 60 \%)$ als weißen Feststoff und das Lactol 156 (1.1 mg, 3.3 mol, 40\%) als hellgelben Feststoff. 
$\mathbf{R}_{\mathbf{f}}=0.31\left(\mathrm{EtOAc} / \mathrm{NEt}_{3}=20: 1\right)$.

${ }^{1} \mathbf{H}$-NMR $\left(300 \mathrm{MHz}, \mathrm{CDCl}_{3}, \mathrm{Z}\right.$-/E-Isomer $\left.=0.5: 0.5\right): \delta=1.04-1.17\left(\mathrm{~m}, 3 \mathrm{H}, \mathrm{CH}_{2} \mathrm{CH}_{3}\right)$, 1.72-1.87 (m, 1.5 H, 2'-H, Isomer A und 2'- $\mathrm{H}_{\mathrm{a}}$, Isomer B), $2.62\left(\mathrm{~m}_{\mathrm{c}}, 0.5 \mathrm{H}, 2^{\prime}-\mathrm{H}_{\mathrm{b}}\right.$, Isomer B), $2.89\left(\mathrm{~s}_{\mathrm{br}}, 1 \mathrm{H}, \mathrm{OH}\right), 3.50\left(\mathrm{~s}, 1.5 \mathrm{H}, \mathrm{OCH}_{3}\right.$, Isomer A), $3.54\left(\mathrm{~s}, 1.5 \mathrm{H}, \mathrm{OCH}_{3}\right.$, Isomer B), 4.65 (dd, ${ }^{3} J=1.4 \mathrm{~Hz},{ }^{2} J=17.1 \mathrm{~Hz}, 0.5 \mathrm{H}, \mathrm{CH}_{\mathrm{a}} \mathrm{H}_{\mathrm{b}} \mathrm{OH}$, Isomer A), 4.77-4.95 (m, 2.5 H, $\mathrm{CH}_{\mathrm{a}} \mathrm{H}_{\mathrm{b}} \mathrm{OH}$, Isomer $\mathrm{A}$ und $\left.\mathrm{CHOCH}_{3}\right), 5.25(\mathrm{~s}, 2 \mathrm{H}, 11-\mathrm{H}), 7.19$ (s, $0.5 \mathrm{H}, 6-\mathrm{H}$, Isomer A), $7.32(\mathrm{~s}, 0.5 \mathrm{H}$, 6-H, Isomer B), $7.63\left(\mathrm{~m}_{\mathrm{c}}, 1 \mathrm{H}, 2-\mathrm{H}\right), 7.81\left(\mathrm{~m}_{\mathrm{c}}, 1 \mathrm{H}, 3-\mathrm{H}\right), 7.92\left(\mathrm{~d},{ }^{3} \mathrm{~J}=8.1 \mathrm{~Hz}, 1 \mathrm{H}, 1-\mathrm{H}\right), 8.20$ (d, $\left.{ }^{3} \mathrm{~J}=8.5 \mathrm{~Hz}, 1 \mathrm{H}, 4-\mathrm{H}\right), 8.35$ (s, $\left.1 \mathrm{H}, 12-\mathrm{H}\right)$.

MS (ESI): $m / z(\%)=349.1(100)[\mathrm{M}+\mathrm{H}]^{+}$.

$\mathbf{C}_{21} \mathbf{H}_{20} \mathbf{N}_{2} \mathbf{O}_{3}(348.40)$

Ber.: $\quad 349.1546 \quad[\mathrm{M}+\mathrm{H}]^{+}$

Gef.: $\quad 349.1547$ (ESI-HRMS)

\subsection{4-Ethyl-3-hydroxy-1,3,4,12-tetrahydro-2-oxa-6,12a-diaza- dibenzo[b,h]-fluoren-13-on (156)}

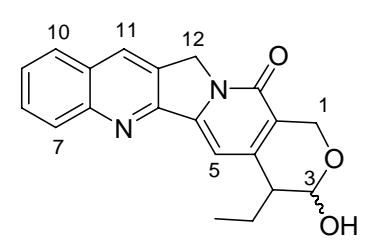

Ausgehend von dem Alkohol 154

(Durchführung siehe $\mathbf{6 . 1 2}$ bei der Synthese von 155)

Ausgehend von dem Aldehyd 158

$\mathrm{Zu}$ einer Suspension des Aldehyds $158\left(0.4 \mathrm{mg}, 1 \mu \mathrm{mol}, 1\right.$ Äq.) in Methanol $(1 \mathrm{~mL})$ bei $0{ }^{\circ} \mathrm{C}$ wurde $\mathrm{NaBH}_{4}$ (1mg) gegeben. Nach 30 min Rühren bei dieser Temperatur wurde die Reaktion durch Zugabe von wässriger 1\%-HCl-Lösung $(2 \mathrm{~mL})$ beendet. Die wäßrige Phase wurde mit $\mathrm{CH}_{2} \mathrm{Cl}_{2}(3 \times 5 \mathrm{~mL})$ extrahiert. Die vereinigten organischen Phasen wurden über $\mathrm{MgSO}_{4}$ getrocknet und filtriert. Nach Entfernung des Lösungsmittels erhielt man das Lactol 156 (0.4 mg, 1 mol, quant.) 
$\mathbf{R}_{\mathbf{f}}=0.13\left(\mathrm{EtOAc}_{\mathrm{NEt}}=20: 1\right)$ oder $0.22(\mathrm{EtOAc} / \mathrm{MeOH}=40: 1)$.

${ }^{1} \mathbf{H}-\mathrm{NMR}\left(300 \mathrm{MHz}, \mathrm{CDCl}_{3}\right): \delta=1.07\left(\mathrm{t}, J=7.5 \mathrm{~Hz}, 3 \mathrm{H}, \mathrm{CH}_{2} \mathrm{CH}_{3}\right), 1.80\left(\mathrm{~m}_{\mathrm{c}}, 1 \mathrm{H}, \mathrm{CH}_{2} \mathrm{CH}_{3}\right)$, $2.67\left(\mathrm{t},{ }^{3} \mathrm{~J}=6.6 \mathrm{~Hz}, 1 \mathrm{H}, 4-\mathrm{H}\right), 2.97\left(\mathrm{~s}_{\mathrm{br}}, 1 \mathrm{H}, \mathrm{OH}\right), 4.85\left(\mathrm{~m}_{\mathrm{c}}, 2 \mathrm{H}, 1-\mathrm{H}\right), 5.24(\mathrm{~s}, 2 \mathrm{H}, 12-\mathrm{H})$, 5.35-5.44 (m, 1 H, 3-H), 7.19 (s, 1 H, 5-H),7.63 (m, 1 H, 9-H), 7.80 (m, 1 H, 8-H), 7.91 (d, $\left.{ }^{3} J=8.3 \mathrm{~Hz}, 1 \mathrm{H}, 10-\mathrm{H}\right), 8.20\left(\mathrm{~d},{ }^{3} J=8.6 \mathrm{~Hz}, 1 \mathrm{H}, 7-\mathrm{H}\right), 8.34$ (s, $\left.1 \mathrm{H}, 11-\mathrm{H}\right)$.

MS (ESI): $m / z(\%)=335.1(100)[\mathrm{M}+\mathrm{H}]^{+}$.

$\mathrm{C}_{20} \mathrm{H}_{18} \mathbf{N}_{2} \mathbf{O}_{3}(334.37)$

Ber.: $\quad 335.1390 \quad[\mathrm{M}+\mathrm{H}]^{+}$

Gef.: $\quad 335.1390 \quad($ ESI-HRMS)

\subsection{2-(9-Oxo-8-methyloxycarbonyl-5b,6,7,8,9,11-hexahydro-} indolizino[1,2-b]-chinolin-7-yl)-buttersäure-tert-butylester (161)

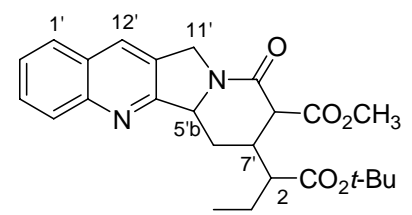

$\mathrm{Zu}$ einer Suspension von 147 (41 mg, $0.11 \mathrm{mmol}, 1.0$ Äq.) und DMAP (3.9 mg, $32 \mu \mathrm{mol}$, 0.30 Äq.) in tert-BuOH $(1.5 \mathrm{~mL})$ bei $\mathrm{RT}$ wurde $\mathrm{Boc}_{2} \mathrm{O}(46 \mu \mathrm{L}, 0.21 \mathrm{mmol}, 2.0$ Äq.) zugetropft. Es wurde bei dieser Temperatur für $20 \mathrm{~h}$ gerührt. Anschließend wurde innerhalb von $5 \mathrm{~h} \mathrm{Boc}_{2} \mathrm{O}(0.2 \mathrm{~mL}, 0.93 \mathrm{mmol}, 8.5 \mathrm{Äq}$ ) in 4 Portionen gegeben. Das Lösungsmittel wurde unter vermindertem Druck entfernt und der Rückstand durch Säulenchromatographie auf Kieselgel (Pentan/EtOAc $=1: 1)$ gereinigt. Man erhielt 161 (24 mg, $54 \mu \mathrm{mol}, 50 \%)$ als farbloses Öl.

$\mathbf{R}_{\mathbf{f}}=0.32(\operatorname{Petan} / \mathrm{EtOAc}=1: 1)$.

MS (ESI): $m / z(\%)=439.2(100)[\mathrm{M}+\mathrm{H}]^{+}$.

$\mathrm{C}_{25} \mathbf{H}_{30} \mathbf{N}_{2} \mathbf{O}_{5}(438.52)$

Ber.: $\quad 439.2227 \quad[\mathrm{M}+\mathrm{H}]^{+}$

Gef.: $\quad 439.2226$ (ESI-HRMS) 


\subsection{2-(8-Methyloxycarbonyl-9-oxo-9,11-dihydro-indolizino[1,2- b]chinolin-7-yl)-buttersäure-tert-butylester (162)}

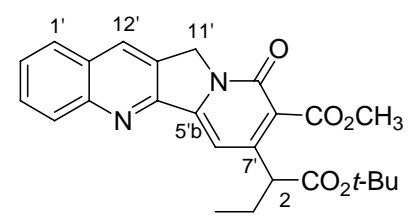

Eine Mischung von 161 (24 mg, 54 mol, 1.0 Äq.) und DDQ (27 mg, 0.12 mmol, 2.2 Äq.) in 1,4-Dioxan $(1.0 \mathrm{~mL})$ wurde bei $70{ }^{\circ} \mathrm{C}$ für $2 \mathrm{~h}$ gerührt, wobei sich Niederschlag bildete. Nach Abkühlung auf RT wurde der Niederschlag abfiltriert und mit $\mathrm{CH}_{2} \mathrm{Cl}_{2}$ gewaschen. Die Mutterlauge wurde im Vakuum konzentriert und der Rückstand durch Säulenchromatographie auf Kieselgel $\left(\mathrm{CH}_{2} \mathrm{Cl}_{2} / \mathrm{EtOAc} / \mathrm{NEt}_{3}=5: 1: 0.25\right)$ gereinigt. Man erhielt den tert-Butylester 162 (9.0 mg, $21 \mu \mathrm{mol}, 38 \%$ ) als gelben Feststoff.

$\mathbf{R}_{\mathbf{f}}=0.21(\operatorname{Petan} / \mathrm{EtOAc}=1: 2)$.

${ }^{1}$ H-NMR (300 MHz, $\left.\mathrm{CDCl}_{3}\right): \delta=0.98\left(\mathrm{t},{ }^{3} \mathrm{~J}=7.4 \mathrm{~Hz}, 3 \mathrm{H}, \mathrm{CH}_{2} \mathrm{CH}_{3}\right), 1.44\left(\mathrm{~s}, 9 \mathrm{H}, \mathrm{C}\left(\mathrm{CH}_{3}\right)_{3}\right)$, 1.88-2.02 (m, $\left.1 \mathrm{H}, 3-\mathrm{H}_{\mathrm{a}}\right), 2.14-2.29\left(\mathrm{~m}, 1 \mathrm{H}, 3-\mathrm{H}_{\mathrm{b}}\right), 3.66\left(\mathrm{t},{ }^{3} \mathrm{~J}=7.5 \mathrm{~Hz}, 1 \mathrm{H}, 2-\mathrm{H}\right), 3.99$ (s, $\left.3 \mathrm{H}, \mathrm{CO}_{2} \mathrm{CH}_{3}\right), 5.23\left(\mathrm{~s}, 2 \mathrm{H}, 11^{\prime}-\mathrm{H}\right), 7.45$ (s, $\left.1 \mathrm{H}, 6^{\prime}-\mathrm{H}\right), 7.64\left(\mathrm{~m}_{\mathrm{c}}, 1 \mathrm{H}, 2^{\prime}-\mathrm{H}\right), 7.81\left(\mathrm{~m}_{\mathrm{c}}, 1 \mathrm{H}\right.$, 3'-H), 7.90 (d, ${ }^{3} J=8.1 \mathrm{~Hz}, 1 \mathrm{H}, 1$ '-H), 8.20 (d, ${ }^{3} \mathrm{~J}=8.6 \mathrm{~Hz}, 1 \mathrm{H}, 4^{\prime}$-H), 8.35 (s, $1 \mathrm{H}, 12^{\prime}$-H). MS (ESI): $m / z(\%)=435.1(100)[\mathrm{M}+\mathrm{H}]^{+}$.

$\mathbf{C}_{25} \mathbf{H}_{26} \mathbf{N}_{2} \mathbf{O}_{5}(434.48)$

Ber.: $\quad 435.1914 \quad[\mathrm{M}+\mathrm{H}]^{+}$

Gef.: $\quad 435.1915$ (ESI-HRMS) 


\subsection{2-(8-Formyl-9-oxo-9,11-dihydro-indolizino[1,2-b]chinolin-7-yl)- buttersäure-tert-butylester (163)}

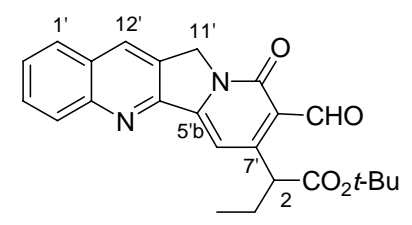

Zu einer Lösung von 162 (8.4 mg, $19 \mu \mathrm{mol}, 1.0$ Äq.) in DME $(0.6 \mathrm{~mL})$ bei $-78^{\circ} \mathrm{C}$ wurde eine verdünnte DIBAL-H-Lösung (0.4 M in Hexan/DME (2:3), $0.15 \mathrm{~mL}, 60 \mu \mathrm{mol}, 3.1$ Äq.) zugetropft. Nach 80 min Rühren bei dieser Temperatur wurde DIBAL-H $(0.4 \mathrm{M}$ in Hexan/DME (2:3), $0.27 \mathrm{~mL}, 0.11 \mathrm{mmol}, 5.6$ Äq.) gegeben und weiter für $75 \mathrm{~min}$ gerührt. Durch Zugabe von $\mathrm{MeOH}(0.5 \mathrm{~mL})$ und Wasser $(2 \mathrm{~mL})$ wurde die Reaktion beendet. Nach Erwärmung auf RT wurde die Reaktionsmischung mit wässriger 5\%-HCl-Lösung (5 mL) versetzt und die wäßrige Phase wurde mit $\mathrm{CH}_{2} \mathrm{Cl}_{2}(3 \times 10 \mathrm{~mL})$ extrahiert. Die vereinigten organischen Phasen wurden mit wässriger $5 \%-\mathrm{NaHCO}_{3}$-Lösung gewaschen, über $\mathrm{Na}_{2} \mathrm{SO}_{4}$ getrocknet und filtriert. Nach Entfernung des Lösungsmittels wurde der Rückstand durch Säulenchromatographie auf Kieselgel $\quad\left(\mathrm{CH}_{2} \mathrm{Cl}_{2} / \mathrm{EtOAc}=3: 1 \rightarrow \mathrm{CH}_{2} \mathrm{Cl}_{2} / \mathrm{MeOH}=15: 1\right)$ gereinigt. Man erhielt zwei Fraktionen bzw. den Aldehyd 163 (2.0 mg, $4.9 \mu \mathrm{mol}, 26 \%$ ) als gelben Feststoff und den Alkohol 164 (4.0 mg, $9.8 \mu \mathrm{mol}, 51 \%)$ als gelbes Öl.

$\mathbf{R}_{\mathbf{f}}=0.38(\operatorname{Petan} /$ EtOAc $=1: 2)$.

${ }^{1} \mathbf{H}$-NMR (300 MHz, $\left.\mathrm{CDCl}_{3}\right): \delta=1.00\left(\mathrm{t},{ }^{3} \mathrm{~J}=7.4 \mathrm{~Hz}, 3 \mathrm{H}, \mathrm{CH}_{2} \mathrm{CH}_{3}\right), 1.44\left(\mathrm{~s}, 9 \mathrm{H}, \mathrm{C}\left(\mathrm{CH}_{3}\right)_{3}\right)$, 1.79-1.95 (m, $\left.1 \mathrm{H}, 3-\mathrm{H}_{\mathrm{a}}\right), 2.12-2.30\left(\mathrm{~m}, 1 \mathrm{H}, 3-\mathrm{H}_{\mathrm{b}}\right), 5.06\left(\mathrm{t},{ }^{3} \mathrm{~J}=7.1 \mathrm{~Hz}, 1 \mathrm{H}, 2-\mathrm{H}\right), 5.34$ (s, $\left.2 \mathrm{H}, 11^{\prime}-\mathrm{H}\right), 7.47$ (s, $1 \mathrm{H}, 6$ ' $\left.-\mathrm{H}\right), 7.70\left(\mathrm{~m}_{\mathrm{c}}, 1 \mathrm{H}, 2^{\prime}\right.$-H), 7.85 ( $\left.\mathrm{m}_{\mathrm{c}}, 1 \mathrm{H}, 3^{\prime}-\mathrm{H}\right), 7.95$ (d, ${ }^{3} J=8.1$ Hz, $\left.1 \mathrm{H}, 1^{\prime}-\mathrm{H}\right), 8.26$ (d, $\left.{ }^{3} \mathrm{~J}=8.6 \mathrm{~Hz}, 1 \mathrm{H}, 4^{\prime}-\mathrm{H}\right), 8.44$ (s, $\left.1 \mathrm{H}, 12^{\prime}-\mathrm{H}\right), 10.67$ (s, $\left.1 \mathrm{H}, \mathrm{CHO}\right)$. MS (ESI): $m / z(\%)=405.1(100)[\mathrm{M}+\mathrm{H}]^{+}$.

$\mathrm{C}_{24} \mathbf{H}_{24} \mathbf{N}_{2} \mathbf{O}_{4}(404.46)$

$\begin{array}{lll}\text { Ber.: } & 405.1808 & {[\mathrm{M}+\mathrm{H}]^{+}} \\ & 427.1628 & {[\mathrm{M}+\mathrm{Na}]^{+}} \\ & 443.1367 & {[\mathrm{M}+\mathrm{K}]^{+}} \\ \text {Gef.: } & 405.1809 & (\mathrm{ESI}-\mathrm{HRMS}) \\ & 427.1628 & \\ & 443.1367 & \end{array}$




\subsection{2-(8-Hydroxymethyl-9-oxo-9,11-dihydro-indolizino[1,2-b]chinolin-7- yl)-buttersäure-tert-butylester (164)}

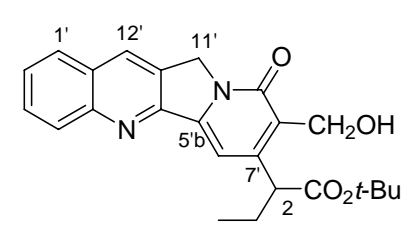

(Durchführung siehe 6.16 bei der Synthese von 163)

$\mathbf{R}_{\mathbf{f}}=0.28(\mathrm{EtOAc} / \mathrm{MeOH}=40: 1)$.

${ }^{1}$ H-NMR (300 MHz, $\left.\mathrm{CDCl}_{3}\right): \delta=0.98\left(\mathrm{t},{ }^{3} \mathrm{~J}=7.4 \mathrm{~Hz}, 3 \mathrm{H}, \mathrm{CH}_{2} \mathrm{CH}_{3}\right), 1.43\left(\mathrm{~s}, 9 \mathrm{H}, \mathrm{C}\left(\mathrm{CH}_{3}\right)_{3}\right)$, 1.82-1.98 (m, $\left.1 \mathrm{H}, 3-\mathrm{H}_{\mathrm{a}}\right), 2.19-2.34\left(\mathrm{~m}, 1 \mathrm{H}, 3-\mathrm{H}_{\mathrm{b}}\right), 3.90\left(\mathrm{t},{ }^{3} J=7.1 \mathrm{~Hz}, 1 \mathrm{H}, 2-\mathrm{H}\right), 4.87\left(\mathrm{~m}_{\mathrm{c}}\right.$, $\left.2 \mathrm{H}, \mathrm{CH}_{2} \mathrm{OH}\right), 5.28\left(\mathrm{~s}, 2 \mathrm{H}, 11^{\prime}-\mathrm{H}\right), 7.47$ (s, $\left.1 \mathrm{H}, 6^{\prime}-\mathrm{H}\right), 7.64\left(\mathrm{~m}_{\mathrm{c}}, 1 \mathrm{H}, 2^{\prime}-\mathrm{H}\right), 7.81\left(\mathrm{~m}_{\mathrm{c}}, 1 \mathrm{H}\right.$, 3'-H), 7.91 (d, ${ }^{3} J=8.1 \mathrm{~Hz}, 1 \mathrm{H}, 1$ ' - $\left.\mathrm{H}\right), 8.22$ (d, $\left.{ }^{3} \mathrm{~J}=8.6 \mathrm{~Hz}, 1 \mathrm{H}, 4^{\prime}-\mathrm{H}\right), 8.36$ (s, $\left.1 \mathrm{H}, 12^{\prime}-\mathrm{H}\right)$. MS (ESI): $m / z(\%)=407.2(100)[\mathrm{M}+\mathrm{H}]^{+}$.

$\mathbf{C}_{24} \mathbf{H}_{26} \mathbf{N}_{2} \mathbf{O}_{4}(406.47)$

$\begin{array}{lll}\text { Ber.: } & 407.1965 & {[\mathrm{M}+\mathrm{H}]^{+}} \\ & 429.1784 & {[\mathrm{M}+\mathrm{Na}]^{+}} \\ & 445.1524 & {[\mathrm{M}+\mathrm{K}]^{+}} \\ \text {Gef.: } & 407.1965 & (\mathrm{ESI}-\mathrm{HRMS}) \\ & 429.1784 & \\ & 445.1524 & \end{array}$




\subsection{0-Desoxycamptothecin (141)}

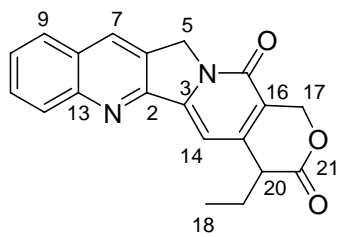

Direkte Synthese von 141 ausgehend von 162

Zu einer Lösung von $162\left(9.0 \mathrm{mg}, 21 \mu \mathrm{mol}, 1.0\right.$ Äq.) in DME $(0.6 \mathrm{~mL})$ bei $-78^{\circ} \mathrm{C}$ wurde eine auf $-78^{\circ} \mathrm{C}$ gekühlte verdünnte DIBAL-H-Lösung (0.4 M in Hexan/DME (2:3), $0.50 \mathrm{~mL}$, $0.20 \mathrm{mmol}$, 9.5 Äq.) innerhalb von $10 \mathrm{~min}$ zugetropft. Nach $50 \mathrm{~min}$ Rühren bei dieser Temperatur wurde die Reaktion durch Zugabe von $\mathrm{MeOH}(0.4 \mathrm{~mL})$, Wasser $(2 \mathrm{~mL})$ und gesättiger Na-K-Tartrat-Lösung ( $2 \mathrm{~mL}$ ) beendet. Nach Erwärmung auf RT wurde die wäßrige Phase wurde mit $\mathrm{CH}_{2} \mathrm{Cl}_{2}(2 \times 15 \mathrm{~mL})$ extrahiert und dann mit Natronlauge $(1 \mathrm{~N}, 3 \mathrm{~mL})$ versetzt. Dann wurde die wässrige Phase wieder mit $\mathrm{CH}_{2} \mathrm{Cl}_{2}(2 \times 5 \mathrm{~mL})$ extrahiert. Die vereinigten organischen Phasen wurden über $\mathrm{Na}_{2} \mathrm{SO}_{4}$ getrocknet, filtriert und im Vakuum konzentriert. Der Rückstand wurde in tert-BuOH $(2 \mathrm{~mL})$ aufgenommen und mit $\mathrm{NaBH}_{4}(2.8$ mg) versetzt. Nach 30 min bei RT wurde die Reaktion durch Zugabe von wässriger 5\%$\mathrm{NaHCO}_{3}$-Lösung $(5 \mathrm{~mL})$ beendet. Die wäßrige Phase wurde mit $\mathrm{CH}_{2} \mathrm{Cl}_{2}(3 \times 15 \mathrm{~mL})$ extrahiert. Die vereinigten organischen Phasen wurden über $\mathrm{Na}_{2} \mathrm{SO}_{4}$ getrocknet, filtriert und im Vakuum konzentriert. Der Rückstand wurde in TFA $(1 \mathrm{~mL})$ aufgenommen und bei RT für 70 min gerührt. Dann wurde Toluol $(25 \mathrm{~mL})$ zur azeotropen Entfernung von TFA gegeben. Nach Entfernung des Lösungsmittels wurde der Rückstand durch Säulenchromatographie auf Kieselgel $\left(\mathrm{CH}_{2} \mathrm{Cl}_{2} / \mathrm{MeOH}=30: 1\right)$ gereinigt. Man erhielt $141(3.3 \mathrm{mg}, 10 \mu \mathrm{mol}, 48 \%)$ als gelben Feststoff.

(Spektroskopische Dateien von 141 siehe 5.6 bei der Synthese von 141) 


\subsection{4-Ethyl-12H-2-oxa-6,12a-diaza-dibenzo[b,h]fluoren-1,13-dion (165)}

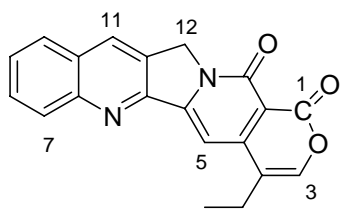

Eine Mischung von 62 (58 mg, 0.16 mmol, 1.0 Äq.) und DDQ (72 mg, 0.32 mmol, 2.0 Äq.) in 1,4-Dioxan $(1.6 \mathrm{~mL})$ wurde bei $55^{\circ} \mathrm{C}$ für $1 \mathrm{~h}$ gerührt, wobei sich Niederschlag bildete. Nach Abkühlung auf RT wurde der Niederschlag abfiltriert und mit 1,4-Dioxan gewaschen. Die Mutterlauge wurde im Vakuum konzentriert und der Rückstand durch Säulenchromatographie auf Kieselgel (EtOAc/ $\left.\mathrm{NEt}_{3}=20: 1\right)$ gereinigt. Man erhielt $165(21 \mathrm{mg}, 64 \mu \mathrm{mol}, 40 \%)$ als hellgelben Feststoff.

$\mathbf{R}_{\mathbf{f}}=0.19\left(\mathrm{EtOAc} / \mathrm{NEt}_{3}=20: 1\right)$

${ }^{1}$ H-NMR (300 MHz, $\left.\mathrm{CDCl}_{3}\right): \delta=1.35\left(\mathrm{t},{ }^{3} \mathrm{~J}=7.5 \mathrm{~Hz}, 3 \mathrm{H}, \mathrm{CH}_{2} \mathrm{CH}_{3}\right), 2.68\left(\mathrm{q}, 1 \mathrm{H}, \mathrm{CH}_{2} \mathrm{CH}_{3}\right)$, 5.39 (s, $2 \mathrm{H}, 12-\mathrm{H}), 7.32\left(\mathrm{~s}, 1 \mathrm{H}, 3-\mathrm{H}^{*}\right), 7.35$ (s, $\left.1 \mathrm{H}, 5-\mathrm{H}^{*}\right), 7.72\left(\mathrm{~m}_{\mathrm{c}}, 1 \mathrm{H}, 9-\mathrm{H}\right), 7.87\left(\mathrm{~m}_{\mathrm{c}}\right.$, $1 \mathrm{H}, 8-\mathrm{H}), 7.97\left(\mathrm{~d},{ }^{3} \mathrm{~J}=8.1 \mathrm{~Hz}, 1 \mathrm{H}, 10-\mathrm{H}\right), 8.27$ (d, $\left.{ }^{3} \mathrm{~J}=8.5 \mathrm{~Hz}, 1 \mathrm{H}, 7-\mathrm{H}\right), 8.43$ (s, $1 \mathrm{H}$, 11-H).

MS (ESI): $m / z(\%)=353.0(20)[\mathrm{M}+\mathrm{Na}]^{+}, 331.1(100)[\mathrm{M}+\mathrm{H}]^{+}$.

$\mathrm{C}_{20} \mathrm{H}_{14} \mathbf{N}_{2} \mathbf{O}_{3}(330.34)$

Ber.:

$331.1077 \quad[\mathrm{M}+\mathrm{H}]^{+}$

$353.0896[\mathrm{M}+\mathrm{Na}]^{+}$

Gef.: $\quad 331.1078$ (ESI-HRMS)

353.0897 


\section{ANHANG}

\section{Verwendete Abkürzungen und Akronyme}

Ac

APT

aq.

Äq.

Ber.

$\mathrm{Bn}$

$(\mathrm{BOC})_{2} \mathrm{O}$

bzg.

bzw.

ca.

CAN

$\mathrm{Cbz}$

$\mathrm{Cbz}-\mathrm{Cl}$

COSY

CPT

$d r$

DC

DCC

DCE

DCI

DDQ

DIBAL-H

DMAP

DME

DMF

DMSO

DNA

EDDA

EI

ESI

Et

EtOAc

$\mathrm{EtOH}$

ee

EWG
Acetyl

attached proton test

wässrig(e)

Äquivalente

Berechnet

Benzyl

Di-tert-Butyldicarbonat

bezogen

beziehungsweise

circa

Ammoniumcer-IV-nitrat $\left(\mathrm{NH}_{4}\right)_{2}\left[\mathrm{Ce}\left(\mathrm{NO}_{3}\right)_{6}\right]$

Benzyloxycarbonyl

Benzylchlorformiat

Correlation Spectroscopy

Camptothecin

Diastereomerenverhältnis (diastereomeric ratio)

Dünnschichtchromatographie

Dicyclohexylcarbodiimid

1,2-Dichlorethan

Direkte Chemische Ionisation

2,3-Dichlor-5,6-dicyano-1,4-benzochinon

Diisobutylaluminiumhydrid

4-Dimethylaminopyridin

1,2-Dimethoxyethan

$N, N$ '-Dimethylformamid

Dimethylsulfoxid

Desoxyribonukleinsäure

Ethylendiammoniumdiacetat

Elektronenstoß-Ionisation

Elektronenspray-Ionisation

Ethyl

Essigsäureethylester

Ethanol

Enantiomerenüberschuss (enantiomeric excess)

Elektronenziehende Gruppe 
FMO

Gef.

gesätt.

$\mathrm{h}$

HOAc

HOMO

HRMS

$\mathrm{Hz}$

i

IR

kat.

KHMDS

konz.

LDA

LHMDS

LUMO

m

$\mathrm{Me}$

$\mathrm{MeOH}$

min

MO

$\mathrm{MMTPCl}$

MMTPBr

MS $3 \AA$

MS

NMR

NOESY

o

$p$

PG

$\mathrm{Ph}$

PMB

$p$-TSA

quant.

$\mathrm{R}_{\mathrm{f}}$
Grenzmolekülorbital (Frontier Molecular Orbital)

gefunden

gesättigt

Stunde(n)

Essigsäure

besetztes Molekülorbital mit der höchsten Energie

(Highest Occupied Molecular Orbital)

hoch aufgelöstes Massenspektrum

Hertz

iso

Infrarot-Spektroskopie

katalytische Mengen

Kalium-bis(trimethylsilyl)amid

konzentriert(e)

Lithiumdiisopropylamin

Lithium-bis(trimethylsilyl)amid

unbesetztes Molekülorbital mit der niedrigsten Energie

(Lowest Unoccupied Molecular Orbital)

meta

Methyl

Methanol

Minute(n)

Molecular Orbital

Methoxymethyltriphenylphosphoniumchlorid

Methoxymethyltriphenylphosphoniumbromid

Molsieb $3 \AA$

Massenspektrometrie

Kernmagnetische Resonanz (Nuclear Magnetic

Resonance)

Kern-Overhauser-Effekt-Spektroskopie (Nuclear

Overhauser Effect Spectroscopy)

ortho

para

Schutzgruppe (Protectiv Group)

Phenyl

para-Methoxybenzyl

para-Toluolsulfonsäure

quantitativ

Retentionswert der Dünnschichtchromatographie 


$\begin{array}{ll}\text { RT } & \text { Raumtemperatur } \\ \text { Sdp. } & \text { Siedepunkt } \\ t & \text { tert } \\ \text { TBACl } & \text { Tetra-n-butylammoniumchlorid } \\ \text { TBAI } & \text { Tetra-n-butylammoniumiodid } \\ \text { tert } & \text { tertiär } \\ \text { TFA } & \text { Trifluoressigsäure } \\ \text { THF } & \text { Tetrahydrofuran } \\ \text { TMS-Cl } & \text { Chlortrimethylsilan } \\ \text { TMS-CHN } & \text { Trimethylsilyldiazomethan } \\ \text { Troc } & 2,2,2-\text { Trichlorethyloxycarbonyl } \\ \text { Troc-Cl } & 2,2,2-\text { Trichlorethylchlorformiat } \\ \text { UV } & \text { Ultraviolett-Spekroskopie } \\ \text { z. B. } & \text { zum Beispiel } \\ * & \text { Zuordnung unsicher } \\ \text { )) } & \text { Ultraschall } \\ \Delta & \text { Erwärmung }\end{array}$




\section{Literaturverzeichnis}

1 F. Wöhler, Ann. Phys. Chem. 1828, 12, 253.

2 K. C. Nicolaou, E. J. Sorensen, Classics in Total Synthesis, VCH, Weinheim, 1996.

3 (a) R. W. Armstrong, J.-M. Beau, S. H. Cheon, W. J. Christ, H. Fujioka, W.-H. Ham, L. D. Hawkins, H. Jin, S. H. Kang, Y. Kishi, M. J. Martinelli, W. W. McWhorter, M. Mizuno, M. Nakata, A. E. Stutz, F. X. Talamas, M. Taniguchi, J. A. Tino, K. Udea, J. Uenishi, J. B. White, M. Yonaga, J. Am. Chem. Soc. 1989, 111, 7525-7530; (b) R. W. Armstrong, J.-M. Beau, S. H. Cheon, W. J. Christ, H. Fujioka, W.-H. Ham, L. D. Hawkins, H. Jin, S. H. Kang, Y. Kishi, M. J. Martinelli, W. W. McWhorter, M. Mizuno, M. Nakata, A. E. Stutz, F. X. Talamas, M. Taniguchi, J. A. Tino, K. Udea, J. Uenishi, J. B. White, M. Yonaga, J. Am. Chem. Soc. 1989, 111, 7530-7533; (c) Y. Kishi, Pure Appl. Chem. 1989, 61, 313-324; d) E. M. Suh, Y. Kishi, J. Am. Chem. Soc. 1994, 116, 11205-11206.

4 (a) K. C. Nicolaou, E. A. Theodorakis, F. P. J. T. Rutjes, J. Tiebes, M. Sato, E. Untersteller, X.-Y. Xiao, J. Am. Chem. Soc. 1995, 117, 1171-1172; (b) K. C. Nicolaou, F. P. J. T. Rutjes, E. A. Theodorakis, J. Tiebes, M. Sato, E. Untersteller, J. Am. Chem. Soc. 1995, 117, 1173-1174; (c) K. C. Nicolaou, F. P. J. T. Rutjes, E. A. Theodorakis, J. Tiebes, M. Sato, E. Untersteller, J. Am. Chem. Soc. 1995, 117, 10252-10263.

5 (a) D. A. Evans, M. R. Wood, B. W. Trotter, T. I. Richardson, J. C. Barrow, J. L. Katz, Angew. Chem. 1998, 110, 2864-2868; Angew. Chem. Int. Ed. 1998, 37, 2700-2704; (b) D. A. Evans, C. J. Dinsmore, P. S. Watson, M. R. Wood, T. I. Richardson, B. W. Trotter, J. L. Katz, Angew. Chem. 1998, 110, 2868-2872; Angew. Chem. Int. Ed. 1994, 37, 2704-2708; (c) K. C. Nicolaou, S. Natarajan, H. Li, N. F. Jain, R. Hughes, M. E. Solomon, J. M. Ramanjulu, C. N. C. Boddy, M. Takayanagi, Angew. Chem. 1998, 110, 2872-2878; (d) K. C. Nicolaou, N. F. Jain, S. 4 Natarajan, R. Hughes, M. E. Solomon, H. Li, J. M. Ramanjulu, M. Takayanagi, A. E. Koumbis, T. Bando, Angew. Chem. 1998, 110, 28792881; (e) K. C. Nicolaou, M. Takayanagi, N. F. Jain, S. Natarajan, A. E. Koumbis, T. Bando, J. M. Ramanjulu, Angew. Chem. 1998, 110, 2881-2883.

6 www.aspirin.de

7 (a) W. J. Slichenmyer, E. K. Rowinsky, R. C. Donehower, S. H. Kaufmann, J. Natl. Cancer I. 1993, 85, 271-291; (b) M. L. Rothenberg, Ann. Oncol. 1997, 8, 837-855; (c) R. Garcia-Carbonero, J. G. Supko, Clin. Cancer Res. 2002, 8, 641-661.

8 (a) L. F. Tietze, U. Beifuß, Angew. Chem. 1993, 105, 137-170; (b) L. F. Tietze, Chem. Rev. 1996, 96, 115-136. 
9 (a) L. F. Tietze, N. Rackelmann, Pure Appl. Chem. 2004, 76, 1967-1983; (b) L. F. Tietze, J. Heterocyclic Chem. 1990, 27, 47-69; (c) L. F. Tietze, N. Rackelmann, The DominoKnoevenagel-hetero-Diels-Alder Reaction and Related Transformations in "Multicomponent Reactions", (Hrsg.: J. Zhu, O. Bienaymé), Wiley-VCH, Weinheim, 2005, S. 121-168; (d) L. F. Tietze, N. Rackelmann, I. Müller, Chem. Eur. J. 2004, 10, 2722-2731; (e) L. F. Tietze, N. Rackelmann, Z. Naturforsch., B: Chem. Sci. 2004, 468477; (f) L. F. Tietze, J. Bachmann, J. Wichmann, Y. Zhou, T. Raschke, Liebigs Ann./Receuil 1997, 881-886; (g) L. F. Tietze, J. Bachmann, J. Wichmann, O. Burkhardt, Synthesis, 1994, 1185-1194.

10 N. H. Oberlies, D. J. Kroll, J. Nat. Prod. 2004, 67, 129-135.

11 M. E. Wall, M. C. Wani, C. E. Cook, K. H. Palmer, A. T. Mcphail, G. A. Sim, J. Am. Chem. Soc. 1966, 88, 3888-3890.

12 W. Kingsbury, J. Boehm, D. Jakas, K. Holden, S. Hecht, G. Gallagher, M. Caranfa, F. McCabe, L. Faucette, R. Johnson, R. Hertzberg, J. Med. Chem. 1991, 34, 98-107.

13 Y. Hsiang, R. Hertzberg, S. Hecht, L. F. Liu, J. Biol. Chem. 1985, 260, 14873-14878.

14 (a) S. P. Chavan, A. B. Pathak, U. R. Kalkote, Tetrahedron Lett. 2007, 48, 6561-6563; (b) C. Tang, M. Babjak, R. J. Anderson, A. E. Greene, A. Kanazawa, Org. Biomol. Chem. 2006, 4, 3757-3759; (c) R. Peters, M. Althaus, A. Nagy, Org. Biomol. Chem. 2006, 4, 498-509; (d) R. J. Anderson, G. B. Raolji, A. Kanazawa, A. E. Greene, Org. Lett. 2005, 7, 2989-2991; (e) W. Du, Tetrahedron 2003, 59, 8649-8687; (f) H Josien, S. Ko, D. Bom, D. P. Curran, Chem. Eur. J. 1998, 4, 67-83.

15 (a)B. L. Staker, K. Hjerrild, M. D. Feese, C. A. Behnke, A. B. Burgin, L. Stewart, Proc. Natl. Acad. Sci. 2002, 99, 15387-15392; (b) M. R. Redinbo, L. Stewart, P. Kuhn, J. J. Champoux, W. G. J. Hol, Science 1998, 279, 1504-1513; (c) R. P. Hertzberg, M. J. Caranf, S. M. Hecht, Biochemistry 2004, 28, 4629-4638.

16 D. F. S. Kehrer, O. Soepenberg, W. J. Loos, J. Verweij. A. Sparreboom, Anti-Cancer Drugs 2001, 12, 89-105.

17 (a) J. L. Wood, J. M. Fortunak, A. R. Mastrocola, M. Mellinger, P. L. Burk, J. Org. Chem. 1995, 60, 5739-5740; (b) W. D. Kingsbury, J. C. Boehm, D. R. Jakas, K. G. Holden, S. M. Hecht, G. Gallagher, M. J. Caranfa, F. L. McCabe, L. F. Faucette, R. K. Johnson, R. P. Hertzberg, J. Med. Chem. 1991, 34, 98-107.

18 S. Sawada, S. Okajima, R. Aiyama, K. Nokata, T. Furuta, T. Yokokura, E. Sugino, K. Yamaguchi, T. Miyasaka, Chem. Pharm. Bull. 1991, 39, 1446-1454. 
19 (a) D. N. Carney, N. Engl. J. Med. 2002, 346, 126-128; (b) K. Fujiwara, H. Ueoka, K. Kiura, M. Tabata, N. Takigawa, K. Hotta, S. Umemura, K. Sugimoto, T. Shibayama, H. Kamei, S. Harita, N. Okimoto, M. Tanimoto, Cancer Chemother Pharmacol 2006, 57, 757-760; (c) K. Noda, Y. Nishiwaki, M. Kawahara, S. Negoro, T. Sugiura, A. Yokoyama, M. Fukuoka, K. Mori, K. Watanabe. T. Tamura, S. Yamamoto, N. Saijo, N. Engl. J. Med. 2002, 346, 85-91; (d) U. Vanhoefer, A. Harstrick, W. Achterrath, S. Cao, S. Seeber, Y. M. Rustum, J. Chlin. Oncol. 2001, 19, 1501-1518; (e) J. A. Meyerhardt, R. J. Mayer, N. Engl. J. Med. 2005, 352, 476-487; (f) L. B. Saltz, J. V. Cox, C. Blanke, L. S. Rosen, L. Fehrenbacher, M. J. Moore, J. A. Maroun, S. P. Ackland, P. K. Locker, N. Pirotta, G. L. Elfring, L. L. Miller, N. Engl. J. Med. 2000, 343, 905-914.

20 (a) J. F. Pizzolato, L. B. Saltz, The LANCET 2003, 361, 2235-2241; (b) H. Ulukan, P. W. Swaan, Drugs 2002, 62, 2039-2057; (c) C. J. Thomas, N. J. Rahier, S. M. Hecht, Bioorg. Med. Chem. 2004, 12, 1585-1604. (d) siehe Lit. 10.

21 T. G. Burke, Z. Mi, J. Med. Chem. 1994, 37, 40-46.

22 (a) M. C. Wani, P. E. Ronman, L. T. Lindley, M. E. Wall, J. Med. Chem. 1980, 23, 554-560; (b) B. C. Giovanella, H. R. Hinz, A. J. Kozielski, J. S. Stehlin, R. Silber, M. Potmesil, Cancer Res. 1991, 51, 3052-3055.

${ }^{23}$ (a) X. Pan, R. Han, P. Sun, Bioorg. Med. Chem.Lett. 2003, 13, 3739-3741; (b) H. Zhao, C. Lee, P. Sai, Y. H. Choe, M. Boro, A. Pendri, S. Guan, R. B. Greenwald, J. Org. Chem. 2000, 65, 4601-4606; (c) P. V. Paranjpe, S. Stein, P. J. Sinko, Anti-Cancer Drugs 2005, $16,763-775$.

24 (a) R. S. Tangirala, S. Antony, K. Agama, Y. Pommier, B. D. Anderson, R. Bevins, D. P. Curran, Bioorg. Med. Chem. 2006, 14, 6202-6212; (b) N. J. Rahier, B. M. Eisenhauer, R. Gao, S. J. Thomas, S. M. Hecht, Bioorg. Med. Chem. 2005, 13, 1381-1386; (c) O. Lavergne, L. Lesueur-Ginot, F. P. Rodas, P. G. Kasprzyk, J. Pommier, D. Demarquay, G. Prévost, G. Ulibarri, A. Rolland, A.-M. Schiano-Liberatore, J. Harnett, D. Pons, J. Camara, D. C. H. Bigg, J. Med. Chem. 1998, 41, 5410-5419.

${ }_{25}$ (a). M. A. Elban, W. Sun, B. M. Eisenhauer, R. Gao, S. M. Hecht, Org. Lett. 2006, 8, 3513-3516; (b) N. J. Rahier, K. Cheng, R. Gao, B. M. Eisenhauer, S. M. Hecht, Org. Lett. 2005, 7, 835-837; (c). K. Cheng, N. J. Rahier, B. M. Eisenhauer, R. Gao, S. J. Thomas, S. M. Hecht, J. Am. Chem.. Soc. 2005, 127, 838-839.

26 G. Stork, A. G. Schultz, J. Am. Chem. Soc. 1971, 93, 4074-4075.

27 D. P. Curran, H. Liu, J. Am. Chem. Soc. 1992, 114, 5864-5866. 
${ }^{28}$ H. Zhou, G. Liu, Z. Yao, Org. Lett. 2007, 9, 2003-2006.

29 O. Diels, K. Alder, Liebigs Ann. Chem. 1928, 44, 98-122.

30 D. A. Evans, T. B. Dunn, L. Kværnø, André, Beauchemin, B. Raymer, E. J. Olhava, J. A. Mulder, M. Juhl, K. Kagechika, D. A. Favor, Angew. Chem. Int. Ed. 2007, 46, 4698-4703.

31 D. A. Vosburg, C. D. Vanderwal, E. J. Sorensen, J. Am. Chem. Soc. 2002, 124, 45524553 .

32 J. Sauer, Angew. Chem. 1967, 79, 76-94.

33 R. B. Woodward, R. Hoffmann, Die Erhaltung der Orbitalsymmetrie, Verlag Chemie, Weinheim 1970; R. B. Woodward, R. Hoffmann, Angew. Chem. 1969, 81, 797-869.

${ }^{34}$ K. Fukui, Acc. Chem. Res. 1971, 4, 57-64.

35 K. N. Houk, Acc. Chem. Res. 1975, 8, 361-396.

36 R. Schubert, R. Sustmann, Tetrahedron Lett. 1972, 2739-2742.

37 G. Desimoni, P. P. Righetti, E. Selva, G. Tacconi, V. Rigante, M. Specchiraello, Tetrahedron 1977, 33, 2829-2830.

38 R. Sustmann, Pure Appl. Chem. 1974, 40, 569-593.

39 K. N. Houk, J. Am. Chem. Soc. 1973, 95, 4092-4094; K. N. Houk, J. Sims, C. R. Watts, L. J. Luskus, J. Am. Chem. Soc. 1973, 95, 7301-7315.

40 I. Fleming, Grenzorbitale und Reaktionen organischer Verbindungen, VCH, Weinheim, 1979.

41 J. I. Garcia, J. A. Mayoral, L. Salvatella, Acc. Chem. Res. 2000, 33, 658-664.

42 (a) L. F. Tietze, F. Haunert, Domino Reactions in Organic Synthesis. An Approach to Efficiency, Elegance, Ecological Benefit. Economic Advantage and Preservation of Our Resources in Chemical Transformations, in: Stimulating Concepts in Chemistry (Hrsg.: F. Vögtle, J. F. Stoddart, M. Shibasaki), Wiley-VCH, Weinheim, 2000, S. 39-64; (b) K. C. Nicolaou, T. Montagnon, S. A. Snyder, Chem. Commun. 2003, 551-564; (c) siehe auch Lit. 8.

43 A. Bruggink, R. Schoevaart, T. Kieboom, Concepts of Nature in Organic Synthesis: Cascade Catalysis and Multistep Conversions in Concert, Org. Proc. Res. Dev. 2003, 7, $622-640$.

${ }^{44}$ L. F. Tietze, K. M. Sommer, J. Zinngrebe, F. Stecker, Angew. Chem. 2005, 117, 262-264; Angew. Chem. Int. Ed. 2005, 44, 257-259.

45 L. F. Tietze, Y. Zhou, Angew. Chem. 1999, 111, 2076-2078; Angew. Chem. Int. Ed. 1999, 38, 2045-2047. 
${ }^{46}$ L. F. Tietze, N. Rackelmann, G. Sekar, Angew. Chem. 2003, 115, 4386-4389.

47 D. B. Ramachary, N. S. Chowdari, C. F. Barbas III, Angew. Chem. Int. Ed. 2003, 42, 4233-4237.

48 L. F. Tietze, U. Beifuss, M. Ruther, J. Org. Chem. 1989, 54, 3120-3129.

49 L. F. Tietze, J. Wichmann, Angew. Chem. 1992, 104, 1091-1092; Angew. Chem. Int. Ed. Engl. 1992, 31, 1079-1080.

50 N. Rackelmann, Dissertation, Göttingen, 2004.

51 Y. Zhou, Dissertation, Göttingen, 1998.

52 (a) S. K. De, R. A. Gibbs, Tetrahedron Lett. 2004, 45, 8141-8144; (b) J. Qi, J. Ji, C. Yueng, H. Kwong, A. S. C. Chan, Tetrahedron Lett. 2004, 45, 7719-7721.

53 D. S. Reddy, S. A. Kozmin, J. Org. Chem. 2004, 69, 4860-4862.

54 M. Schlosser, B. Schaub, Chimia 1982, 36, 396-397.

55 A. Rohrer, R. Ocampo, H. J. Callot, Synthesis 1994, 45, 923-925.

56 P. J. Belshaw, S. Mzengeza, G. A. Lajoie, Synthetic Commun. 1990, 20, 3157-3160.

${ }^{57}$ C. H. Heathcock, M. C. Pirrung, S. D. Young, J. P. Hagen, E. T. Jarvi, U. Badertscher, H. Märki, S. H. Montgomery, J. Am. Chem. Soc. 1984, 106, 8161-8174.

58 S. P. Chavan, R. Sivappa, Tetrahedron Lett. 2004, 45, 3113-3115.

59 O. Meth-Cohn, B. Narine, B. Tarnowski, J. Chem. Soc., Perkin Trans. I 1981, 1520-1530.

${ }^{60}$ O. Meth-Cohn, B. Narine, B. Tarnowski, J. Chem. Soc., Perkin Trans. I 1981, 2509-2517.

${ }^{61}$ C. Wolf, G. E. Tumambac, C. N. Villalobos, Synlett 2003, 1801-1804.

${ }^{62}$ W. A. Herrmann, C. Brossmer, C. Reisinger, T. H. Riermeier, K. Öfele, M. Beller, Chem. Eur. J. 1997, 3, 1357-1364.

${ }^{63}$ Z. J. Song, M. Zhao, L. Frey, J. Li, L. Tan, C. Y. Chen, D. M. Tschaen, R. Tillyer, E. J. J. Grabowski, R. Volante, P. J. Reider, Org. Lett. 2001, 3, 3357-3360.

64 R. F. Borch, M. D. Bernstein, H. D. Durst, J. Am. Chem. Soc. 1997, 93, 2897-2904.

${ }^{65}$ A. F. Abdel-Magid, K. G. Carson, B. D. Harris, C. A. Maryanoff, R. D. Shah, J. Org. Chem. 1996, 61, 3849-3862.

${ }^{66}$ E. Schenker, Angew. Chem. 1961, 106, 81-107.

${ }^{67}$ M. A. Ciufolini, F. Roschangar, Tetrahedron 1997, 53, 11049-11060.

${ }^{68}$ B. V. Yang, D. O’Rourke, J. Li, Synlett 1993, 195-196.

69 D. Dubé, A. A. Scholte, Tetrahederon Lett. 1999, 40, 2295-2298.

${ }^{70}$ K. Yamada, T. Kurokawa, H. Tokuyama, T. Fukuyama, J. Am. Chem. Soc. 2003, 125, 6630-6631. 
71 R. A. Olofson, J. T. Martz, J. Org. Chem. 1984, 49, 2081-2082.

72 E. Erdir, Tetrahedron 1987, 43, 2203-2212.

73 H. Denzer, Dissertation, Göttingen, 1995.

74 (a) C. S. F. Tang, C. J. Morrow, H. Rapoport, J. Am. Chem. Soc. 1995, 97, 159-167; (b) R. T. Brown, J. Liu, C. A. M. Santos, Tetrahedron Lett. 2000, 41, 859-862; (c) W. Shen, C. A. Coburn, W. G. Bornmann, S. J. Danishefsky, J. Org. Chem. 1993, 58, 611-617.

75 B. S. Bal, W. E. Childers, H. W. Pinnick, Tetrahedron 1981, 37, 2091-2096.

76 M. Schlosser, T. Jenny, Y. Guggisberg, Synlett 1990, 704.

77 (a) E. Kühnel, D. D. P. Laffan, G. C. Lloyd-Jones, T. Martínez del Campo, I. R. Shepperson, J. L. Slaughter, Angew. Chem. Int. Ed. 2007, 46, 7075-7078; (b) A. K. Chakraborti, A. Basak, V. Grover, J. Org. Chem. 1999, 64, 8014-8017.

78 (a) S. Danishefsky, E. Berman, L. A. Clizbe, M. Hirama, J. Am. Chem. Soc. 1979, 101, 4385-4386; (b) E. Morera, F. Pinne, G. Lucente, Org. Lett. 2002, 4, 1139-1142.

79 (a) A. Padwa, M. D. Danca, K. I. Hardcastle, M. S. McClure, J. Org. Chem. 2003, 68, 929-941; (b) D. F. Taber, J. F. Mack, A. L. Rheingold, S. J. Geib, J. Org. Chem. 1989, 54, $3831-3836$.

80 S. Danishefsky, R. Volkmann, Tetrahedron Lett. 1973, 2521-2524.

81 D. P. Curran, H. Liu, H. Josien, S. Ko, Tetrahedron 1996, 52, 11385-11404.

82 T. Brunin, L. Legentil, J. Hénichart, Benoît Rigo, Tetrahedron 2006, 62, 3959-3968.

${ }^{83}$ M. Boch, T. Korth, J. M. Nelke, D. Pike, H. Radunz, E. Winterfeldt, Chem. Ber. 1972, 105, 2126-2142.

${ }^{84}$ (a) K. Tagami, N. Nakazawa, S. Sano, Y. Nagao, Heterocycles 2000, 53, 771-775; (b) F. A. Davis, M. C. Weismilller, J. Org. Chem. 1990, 55, 3715-3717.

85 E. F. DiMauro, M. C. Kozlowski, J. Am. Chem. Soc. 2002, 124, 12668-12669.

${ }^{86}$ siehe Lit. $14 \mathrm{~b}$.

87 (a) R. A. Bell, M. B. Gravestock, Can. J. Chem. 1969, 47, 2099-2102; (b) H. C. Brown, E. J. Mead, J. Am. Chem. Soc. 1953, 75, 6263-6265.

${ }^{88}$ H. Tanaka, T. Kamikubo, N. Yoshida, H. Sakagami, T. Taniguchi, K. Ogasawara, Org. Lett. 2001, 3, 679-681.

89 (a) D. A. Evans, D. J. Adams, J. Am. Chem. Soc. 2007, 129, 1048-1049; (b) M. Bonin, J. Royer, D. S. Grierson, H. -P. Husson, Tetrahedron Lett. 1986, 27, 1569-1572.

90 B. S. J. Blagg, D. L. Boger, Tetrahedron 2002, 58, 6343-6349. 
91 (a) M. -L. Bennasar, E. Zulaica, C. Juan, Y. Alonso, J. Bosch, J. Org. Chem. 2002, 67, 7465-7474; (b) siehe Lit. 74b.

92 siehe Lit. 91a.

93 B. Neises, W. Steglich, Angew. Chem. Int. Ed. Engl. 1978, 17, 522-524.

94 (a) J. Thierry, C. Yue, P. Potier, Tetrahedron Lett. 1998, 39, 1557-1560; (b) A. Armstrong, I. Brackenridge, R. F. W. Jackson, J. M. Kirk, Tetrahedron Lett. 1988, 29, 2483-2486.

95 K. Takeda, A. Akiyama, H. Nakamura, S. Takizawa, Y. Mizuno, H. Takayanagi, Y. Harigaya, Synthesis 2007, 46, 7075-7078

96 (a) J. N. Chatterjea, Chem. Ber. 1958, 91, 2636-2638; (b) J. N. Chatterjea, B. K. Banerjee, H. C. Jha, Chem. Ber. 1965, 98, 3279-3285.

97 D. D. Perrin, W. L. F. Arnarego, Purification of Laboratory Chemicals, $3^{\text {rd }}$ Ed., Pergamon Press, Oxford, 1988. 


\section{Danksagung}

Zunächst möchte ich den Mitarbeitern der NMR-Abteilung bestehend aus: Herrn R. Machinek, Frau C. Zolke usw. für die Aufnahme unzähliger NMR-Spektren danken. Daneben gilt mein Dank Herrn Dr. Holm Frauendorf und Frau G. Udvarnoki für die Messung der Massenspektren. Ebenso danke ich Evelyn Pfeil für die Aufnahme der UV/IR-Spektren. Vielen Dank auch an Martina Pretor, Tom Kinzel für die Hilfe bei Computerproblemen.

Ebenfalls möchte ich mich bei Marcel Alexander Düfert und Michael Müller für das sorgfältige und rechtzeitliche Korrekturlesen dieser Arbeit bedanken. Herzlich danke ich Christian Brazel, Marcel Alexander Düfert, Niels Böhnke, Chrisitian Raith, Florian Stecker, Florian Lotz und Dirk Spiegl für die stetige Hilfsbereitschaft.

Ohne viel Materialhilfe von meinen Kollegen könnte meine Arbeit nicht so reibungslos gehen. Ich bin herzlich Guanghui An und dankbar für alle Abende, an den er mich zur Arbeit begleitet hat.

Zuletzt danke ich besonders meiner Frau für ihre stetige Unterstützung während meiner ganzen Diplomarbeit. 


\section{Lebenslauf}

\section{Persönliche Daten}

Name:

Deshan Liu

Geburtsdatum:

30.12.1976

Geburtsort:

Huaian, Jiangsu Provinz, China

Staatsangehörigkeit: chinesisch

Familienstand:

verheiratet

\section{Schulausbildung}

1983-1988

Huaian Shifan-Schule, Huaian

1988-1994

Huaian-Gymnasium, Huaian

1994-1998

Nankai Universität, Tianjin

\section{Studium}

09/1994-07/1998

Nankai Universität, Tianjin

04/2002-07/2004

Studium der Chemie, Georg-August-Universität Göttingen

03/2004-05/2004

Diplomarbeit am Institut für Organische und Biomolekulare Chemie der Georg-August-Universität zu Göttingen unter der Leitung von Prof. Dr. Dr. h.c. L. F. Tietze über die "Synthese von Aminonaphthalinen unter Verwendung eines Mikroreaktors "

09/07/2004 Diplom-Chemiker-Hauptprüfung

09/2004-03/2008

Dissertation am Institut für Organische und Biomolekulare Chemie der Georg-August-Universität zu Göttingen unter der Leitung von Prof. Dr. Dr. h.c. L. F. Tietze über die "Totalsynthese von Camptothecin"

$07 / 2008$

Mündliche Promotionsprüfung 